\title{
Generalized Parameter Identification for Finite Viscoelasticity
}

\author{
vom Fachbereich Maschinenbau und Verfahrenstechnik \\ der Technischen Universität Kaiserslautern \\ zur Verleihung des akademischen Grades \\ Doktor-Ingenieur (Dr.-Ing.) \\ genehmigte Dissertation
}

\author{
von Dipl.-Ing. Bernd Kleuter \\ aus Düsseldorf
}

Hauptreferent: Prof. Dr.-Ing. habil. P. Steinmann Koreferenten: $\quad$ Prof. Dr.-Ing. habil. S. Reese Prof. Dr.-Ing. habil. R. Mahnken PD Dr.-Ing. habil. A. Menzel

Vorsitzender:

Prof. Dr.-Ing. R. Renz

Dekan:

Prof. Dr.-Ing. J. Aurich

Tag der Einreichung:

10. Januar 2007

Tag der mündlichen Prüfung: $\quad$ 25. Mai 2007

September 2007

D 386 



\section{Vorwort}

Die vorliegende Arbeit entstand während meiner Tätigkeit als wissenschaftlicher Mitarbeiter am Lehrstuhl für Technische Mechanik der Technischen Universität Kaiserslautern im Zeitraum 2003 bis 2007. In diesem Zusammenhang danke ich besonders der Stiftung Rheinland-Pfalz für Innovation für die Finanzierung des Forschungsvorhabens "Viskoelastische Materialien bei großen Verformungen: Modellbildung und Parameteridentifikation".

Bei Herrn Professor Dr.-Ing. habil. Steinmann möchte ich mich für die wissenschaftliche Freiheit bei der Durchführung meiner Arbeit und für die Übernahme des Hauptreferates bedanken. Weiterhin danke ich Herrn Professor Dr.-Ing. habil. R. Mahnken, Frau Professorin Dr.-Ing. habil. S. Reese und Herrn PD Dr.-Ing. habil. Andreas Menzel für die Übernahme der Koreferate und ihr Interesse an meiner Arbeit.

Besonders möchte ich mich bei Frau C.E. Jeblick und Herrn J. Hirsch für Ihre Unterstützung und die freundliche Zusammenarbeit bei den am Lehrstuhl angefallen Arbeiten bedanken. Weiterhin danke ich meinem Bürokollegen Herrn Dr.-Ing. Duc Khoi $\mathrm{Vu}$ für die interessanten Diskussionen.

Bei Herrn Dipl.-Ing. Marc Bosseler möchte ich mich sehr für die experimentellen Untersuchungen und die vielen motivierenden fachlichen Diskussionen bedanken. Ohne diese Zusammenarbeit hätte die vorliegende Arbeit nicht entstehen können. Die Mehrzahl der Experimente wurde im Rahmen des von der Stiftung Rheinland-Pfalz für Innovation ebenfalls geförderten Parallelprojektes "Viskoelastische Materialien bei großen Verformungen: Experiment und Parametererfassung" am Lehrstuhl für Ressourcengerechte Produktentwicklung (RPE) unter der Leitung von Herrn Professor Dr.-Ing. R. Renz durchgeführt, bei dem ich mich hierfür recht herzlich bedanken möchte. Weiterhin danke ich in diesem Zusammenhang Herrn W. Hauth und Herrn Dr.-Ing. G. Stelzer, die durch ihre fachliche Hilfe uns hierbei sehr unterstützt haben.

Mein besonderer Dank gilt jedoch meiner Familie, die mich immer - und besonders während meiner Zeit als wissenschaftlicher Mitarbeiter - unterstützt hat. 



\section{Contents}

1 Introduction 1

2 Finite deformation hyperelasticity 9

2.1 Kinematics . . . . . . . . . . . . . . . . . . . . . . . . 9 9

2.1.1 Eigenvalues and eigenvectors of strain tensors . . . . . . . . . . . 9

2.2 Isotropic hyperelasticity in terms of invariants . . . . . . . . . . . . . 11

2.3 Isotropic hyperelasticity in terms of principle stretches . . . . . . . . . . 12

2.3.1 Constitutive equations in terms of the principal stretches . . . . . . 12

2.3.2 Ogden material model . . . . . . . . . . . . . . . . . . . 13

3 Finite deformation viscoelasticity in principal directions 17

3.1 Kinematics of finite viscoelasticity . . . . . . . . . . . . . . . . 18

3.1.1 Time derivatives . . . . . . . . . . . . . . . . . . . 19

3.2 Constitutive model . . . . . . . . . . . . . . . . . . . . . . . . . . . . . . . 19

3.2.1 Free energy function . . . . . . . . . . . . . . . . . . . . 19

3.2.2 Notes on thermodynamics . . . . . . . . . . . . . . . 20

3.2.3 Evolution equations for multiplicative viscoelasticity . . . . . . . . . 23

3.3 Algorithmic formulation . . . . . . . . . . . . . . . . . . 24

3.3.1 Exponential time integration . . . . . . . . . . . . . . . . . 25

3.3.2 Free energy functions . . . . . . . . . . . . . . . . . 27

3.3.3 Tangent operator . . . . . . . . . . . . . . . . . . . . . . 29

4 Basic methods for parameter identification 33

4.1 The inverse problem . . . . . . . . . . . . . . . . . . . . 33

4.2 Non-contacting optical strain measurement systems . . . . . . . . . . . 34

4.2.1 Image correlation photogrammetry . . . . . . . . . . . . . . . 34

4.2 .2 Video extensometry . . . . . . . . . . . . . . . . . . . 34

4.3 Interpolation of experimental data . . . . . . . . . . . . . . . . . . . . . . . . . . . .

4.3.1 One-dimensional interpolation . . . . . . . . . . . . . . . 35

4.3.2 Two-dimensional interpolation . . . . . . . . . . . . . . 39

4.4 Solution of the inverse problem . . . . . . . . . . . . . . . . . . . . . . . . . . . . 42

4.4.1 The Gauss-Newton method . . . . . . . . . . . . . . . . . 43

4.4 .2 The Levenberg-Marquardt method . . . . . . . . . . . . . . . . 44

4.5 Stability investigations . . . . . . . . . . . . . . . . . . . . . . . . . . . . .

5 Sensitivity Analysis $\quad \mathbf{4 7}$

5.1 Direct differentiation method . . . . . . . . . . . . . . . . . 47

5.1.1 Global solution of the direct problem . . . . . . . . . . . . . 47 
5.1.2 Global solution of the inverse problem . . . . . . . . . . . . . . . 49

5.1.3 Sensitivity analysis for the model of finite viscoelasticity . . . . . . 49

5.2 Finite difference method . . . . . . . . . . . . . . . . . 53

6 Parameter identification on the basis of virtual measurement data 55

6.1 Finite Ogden hyperelasticity . . . . . . . . . . . . . . . . 56

6.2 Finite viscoelasticity with one relaxation term . . . . . . . . . . . . . 59

7 Parameter identification for finite viscoelasticity on the basis of real mea$\begin{array}{ll}\text { surement data } & 63\end{array}$

7.1 Polyurethane adhesive . . . . . . . . . . . . . . . . . 63

7.1.1 Experiments . . . . . . . . . . . . . . 64

7.1.2 Interpolation of experimental data . . . . . . . . . . . . 64

7.1.3 Choice of the model structure and parameter identification process 66

7.2 Closed cell polyurethane . . . . . . . . . . . . . . . . 76

7.2 .1 Experiments . . . . . . . . . . . . . . . . 77

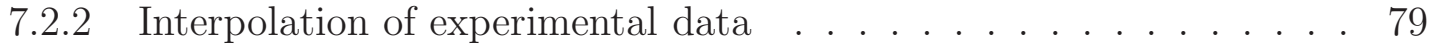

7.2 .3 Least squares problem . . . . . . . . . . . . . . . . . . . . 80

7.2 .4 Identified parameter sets . . . . . . . . . . . . . . . . . 82

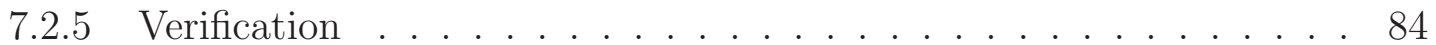

7.3 Compact polyurethane . . . . . . . . . . . . . . . . . 96

7.3.1 Experiments . . . . . . . . . . . . . . 96

7.3.2 Interpolation of experimental data . . . . . . . . . . . . . . 98

7.3 .3 Least squares problem . . . . . . . . . . . . . . . . . 100

7.3.4 Choice of the model structure . . . . . . . . . . . . . . . . . . . 101

7.3.5 Parameter identification process . . . . . . . . . . . . . . . 102

7.3 .6 Verification . . . . . . . . . . . . . . . . . 105

7.3 .7 Extended verification . . . . . . . . . . . . . . 116

8 Parameter identification for plasticity on the basis of real measurement data of aluminum alloy AISi9Cu3

8.1 Constitutive equations for von Mises plasticity . . . . . . . . . . . . . . . 121

8.2 Specimens . . . . . . . . . . . . . . . . . . . . . . . . 123

8.3 Experiments . . . . . . . . . . . . . . . . . . . . 124

8.4 Interpolation of experimental data . . . . . . . . . . . . . . . 125

8.5 Parameter identification by means of relative displacements . . . . . . . . 125

8.6 Parameter identification for experimental results of $\mathrm{AlSi9Cu} 3 \ldots$. . . . . . 128

8.6.1 Identification for the combination of the tests of the specimens P83,

P84, and P85 . . . . . . . . . . . . . . . . . . 128

8.6.2 Individual identification for P83 . . . . . . . . . . . . . . . 137

8.6.3 Individual identification for P84 . . . . . . . . . . . . . . . . . 140

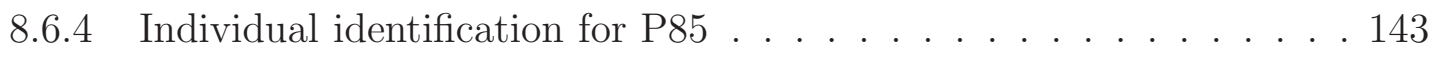

8.6.5 Concluding remarks . . . . . . . . . . . . . . . . . . . 146

9 Summary and conclusions 
A Derivatives

151

A.1 Calculation of $\partial\left(\boldsymbol{n}_{\mathrm{a}} \otimes \boldsymbol{n}_{\mathrm{a}}\right) / \partial \boldsymbol{b}$

151

Bibliography 



\section{Introduction}

Due to the importance of elastomer components in engineering applications the accurate prediction of the mechanical behavior under operational conditions is a relevant subject in industry. Especially the designing with advanced or new materials necessitates the knowledge of their exact stress and strain properties. Therefore, - with the increasing performance of computers - increasingly more complex material models can be applied whose material parameters must be identified for the respective material in order to establish reliability of the simulation. Often the material is exposed to different short- and long-time loads simultaneously. Thus the requirement is to provide one set of parameters for a material law which represents the material behavior over the entire time range of interest sufficiently accurate. Moreover, in many cases the mechanical properties of the applied material scatters for different charges of the production. Then, the requirement for a simulation might be to predict the mechanical behavior by means of averaged material parameters for these different material charges.

This work presents the procedures for the parameter identification for multiple experiments in which all parameters are identified simultaneously. Based on a least squares sum we aim at minimizing the discrepancies between measured and simulated inhomogeneous displacement fields.

Despite the Mullins effect - the stress softening in the first load cycle(s) -, in most elastomeric materials no further damage can be observed even at large deformations. Moreover vulcanized rubber, its synthetic substitutes and most other elastomeric materials do not exhibit considerable permanent (irreversible) deformations. Considering pre-damaged material which is in a stable stationary state with respect to the Mullins effect, this leads to the choice of a viscoelastic material model at large strains. Generally the choice of the material law is a very important issue. For the achievement of a good agreement between simulated and measured data, the model and its structure has to be chosen so that the considered relaxation processes of the material can be described properly. Moreover, in order to simulate the viscoelastic material behavior of typical rubber-like materials, large deformations and large deviations away from thermodynamic equilibrium have to be considered. In 1998 Reese and Govindjee [65] proposed a continuum formulation which is, in contrast to many other theories, not restricted to viscoelastic response for states near the thermodynamic equilibrium (for further elaborations see, e.g. Reese and Govindjee [64], Govindjee and Reese [18], Lion [34], [35], Haupt [21], Holzapfel [23] and Huber and Tsakmakis [25]).

In recent years the identification of the material parameters for viscoelastic constitutive laws has additionally been investigated. Naming but a few research groups which placed emphasis on these aspects, the reader is referred to the contributions by, for instance, Haupt and Sedlan [22], Miehe and Keck [57] Scheday and Miehe [67], Keck [28] as well as to Hartmann et al. [20]. A micromechanically motivated model for viscoelastic response 
has been elaborated in the work by Scheday [66], wherein material parameters have been identified for inhomogeneous deformation fields based on nonlinear finite-element techniques combined with experimental measurements by image correlation photogrammetry. The common technique for the parameter identification for modular constitutive laws is to split the parameters into different sets which are each identified individually by one test. In doing so, in order to identify the parameters of the equilibrium part, the experiments have to deliver test data with fully completed stress relaxation. Thereafter the material parameters of the non-equilibrium term(s) often are identified by a single test where the viscous stress relaxation is investigated over a chosen time period while the parameters of the equilibrium term are fixed.

The reason for the simultaneous identification for multiple tests arises from different facts. First an insufficient number of testing data can cause instable or non-unique parameter estimates. If the data is incomplete it cannot cover the whole range of intended model applications (see Mahnken [40]). Secondly the identification process shall average the parameters respecting different sources of error. Parameter identification is an optimization problem where the discrepancies between test data and simulated data might not vanish completely which is due to two main problems: On the one hand, the chosen mathematical model cannot completely describe the mechanical behavior of a material, thus the model error (see Mahnken [40]) has to be taken into account. On the other hand, uncertainties in laboratory tests occur. This means - as mentioned above - that in addition to possible errors in the measurements themselves, scattering of data due to varying properties of the samples might occur. Therefore, by simultaneous identification an optimal set of parameters in the sense of an optimal adjustment of the constitutive model - being subject to a model error itself - to all tests is achieved. Moreover, the consideration of all tests in one optimization iteration leads to an improved adjustment of the imbalances which arise from scattering of experimental data.

For representative simulations, the viscoelastic material law introduced by Reese and Govindjee [65] with multiple relaxation mechanisms (see Govindjee and Reese [18]) is applied by means of the finite-element method (FEM). Following the approach advocated by Mahnken and Stein ( [48], [47], [49], and Mahnken [40], [42]), a sensitivity analysis which respects the inhomogeneity of the displacement fields is performed. The displacement fields in the experiments are determined by means of optical contactless measurement techniques. The simulation of the experiments is force-controlled with the force-time data measured in the experiments.

The work is organized as follows: In chapter 2 essential equations for the description of finite deformation hyperelasticity for isotropy by means of nonlinear continuum mechanics are listed. Considering isotropic hyperelasticity in terms of principle stretches Ogden-type functions (see Ogden [59]) are discussed briefly.

The Ogden strain energy function is applied for the constitutive routine for finite viscoelasticity (see Reese and Govindjee [65]). The basic constitutive equations of this inelastic material law are reviewed in chapter 3.

The objective of chapter 4 is to present the basic methods within a parameter identification process. First, the definition of the inverse problem is discussed. Afterwards, optical measurement techniques for the measurement of displacement fields and the interpolation of measured displacement fields are described. Then the methods, which are used for 
the numerical solution of the inverse problem in this work, are presented and stability problems - usually occurring during the identification process - are investigated. In the following chapter 5 , the computation of the derivatives required for a sensitivity analysis is presented.

In order to estimate the stability behavior of the viscoelastic law within parameter identifications, two numerical examples for re-identification problems are presented in chapter 6.

In chapter 7 the experimental observations on different elastomeric materials and the respective parameter identification for the finite viscoelastic material are considered.

In section 7.1 the parameter identification for a cured polyurethane adhesive is presented, whereby only the short time viscoelastic behavior - with one relaxation term for the material model - shall be investigated by taking into account the experimental data of a single relaxation test.

In section 7.2 we present an algorithm for the identification of material parameters for the viscoelastic material law with one relaxation term, in which data of three different experiments are considered. The material is a polyurethane foam which is investigated for compression tests.

Section 7.3 is concerned with the parameter identification for a compact polyurethane within different time ranges. The basic observations made in a short-time and a longtime relaxation test and a short-time test with one load cycle motivate the choice of the material model structure with two relaxation modules for the simulation. Applying the method of simultaneous identification for multiple tests, the experimental loading intervals for long-time experiments can be shortened in time and the parameter identification procedure is now referred to experimental data of tests under short- and long-time loads without separating the parameters due to these different time scales. By verification it can be shown that the proposed procedure delivers a very good agreement between experimental and simulated data for all investigated tests. Moreover, as the viscoelastic behavior for long-time intervals is considered, with this approach time-consuming tests with fully completed stress relaxation are no longer required (see also Kleuter et al. [30]). In order to show the general applicability of the identification method for multiple tests, in chapter 8 the parameter identification for small strain plasticity is presented. Thereby three similar test programs on three specimen of the aluminum alloy AlSi9Cu3 are analyzed, and the parameter sets for the respective individual identifications, and for the combination of all tests in one identification, is compared. 


\section{Einleitung}

Aufgrund der großen Bedeutung von Elastomer-Komponenten im Maschinenbau und der Bauindustrie ist die genaue Vorhersage ihres mechanischen Verhaltens unter Betriebsbedingungen eine wichtige Aufgabenstellung in der Industrie. Besonders die Bauteilauslegung mit hochentwickelten oder neuen Materialien erfordert die Kenntnis ihres genauen Spannungs- und Verzerrungsverhaltens. Für die Simulation der Bauteile werden - vor allem aufgrund der zunehmenden Rechenleistung - immer komplexere Materialmodelle eingesetzt. Um die Zuverlässigkeit der Simulation gewährleisten zu können, müssen die zugehörigen Materialparameter identifiziert werden. Oftmals wird das Elastomermaterial verschiedenen Kurz- und Langzeitbelastungen gleichzeitig ausgesetzt. Somit ist die Anforderung an die Parameteridentifikation, einen Parametersatz zu liefern, so dass die Simulation das Materialverhalten über den gesamten zu betrachtenden Zeitraum genügend genau beschreiben kann. Oftmals soll bei der Simulation berücksichtigt werden, dass das mechanische Verhalten über verschiedene Produktionschargen streut. Dann ist die Anforderung an die Identifikation, einen Satz von Materialparametern zu liefern, der eine Mittelung bezüglich dieser Streuungen darstellt.

Diese Arbeit stellt die Prozeduren dar, die für die Parameteridentifikation für mehrere Versuche bei gleichzeitiger Identifikation aller Materialparameter notwendig sind. Basierend auf der Methode der kleinsten Fehlerquadrate ist es das Ziel, die Unterschiede zwischen gemessenen und simulierten inhomogenen Verschiebungsfeldern zu minimieren.

Neben dem Mullins-Effekt, dem Spannungsentfestigungseffekt in den ersten Lastzyklen, kann bei den meisten Elastomer-Materialien - sogar bei sehr großen Deformationen - kein weiterer Einfluß von Schädigungseffekten bemerkt werden. Weiterhin weisen vulkanisierter Kautschuk, synthetisches Gummi und die meisten weiteren Elastomere keine nennenswerten permanenten (irreversiblen) Deformationen auf. Wird bezüglich des Mullins-Effekts vorgeschädigtes Material untersucht, führt dies zu der Auswahl eines viskoelastischen Materialmodells. Generell ist die Auswahl des Materialgesetzes eine sehr wichtige Problemstellung. Um eine gute Übereinstimmung zwischen simulierten und gemessenen Daten zu erhalten, muß das Materialgesetz und die zugehörige Struktur so gewählt werden, dass die betrachteten Relaxationsprozesse des Materials hinreichend genau beschrieben werden können. Darüber hinaus müssen für die Simulation des viskoelastischen Materialverhaltens typischer gummiähnlicher Materialien große Deformationen und große Abweichungen vom thermodynamischen Gleichgewicht berücksichtigt werden. Von Reese and Govindjee [65] wurde 1998 eine neue Kontinuumsformulierung vorgeschlagen, die im Gegensatz zu vielen anderen Theorien nicht auf kleine Abweichungen vom thermodynamischen Gleichgewicht beschränkt ist, siehe auch Reese und Govindjee [64], Govindjee und Reese [18], Lion [34], [35], Haupt [21], Holzapfel [23] sowie Huber und Tsakmakis [25].

In den letzten Jahren wurde ebenfalls die Identifikation von Materialparametern für visko- 
elastische konstitutive Gesetze untersucht. Um einige Forschungsgruppen zu nennen, die auf diesem Gebiet gearbeitet haben, sei der Leser auf die Arbeiten von zum Beispiel Haupt und Sedlan [22], Miehe und Keck [57], Scheday und Miehe [67], Keck [28] sowie auf Hartmann et al. [20] verwiesen.

Ein mikromechanisch motiviertes Modell für viskoelastisches Antwortverhalten wurde in der Arbeit von Scheday [66] betrachtet, wobei Materialparameter für inhomogene Verschiebungsfelder - basierend auf nichtlinearen Finite-Elemente Techniken in Verbindung mit experimentellen Messungen mit dem Objektrasterverfahren - identifiziert wurden.

Die gewöhnlich zur Parameteridentifikation für modular aufgebaute Stoffgesetze verwendete Technik ist, die Parameter in verschiedene Sätze zu trennen, die separat voneinander jeweils anhand eines Experiments identifiziert werden. Um die Parameter des Gleichgewichtsterms zu identifizieren, müssen die Experimente bei dieser Vorgehensweise Meßdaten für vollständig abgeschlossene Relaxationsvorgänge liefern. Danach werden die Parameter der Nichtgleichgewichtsterme oftmals anhand eines einzigen Experiments identifiziert, wobei die viskose Spannungsrelaxation über eine gewählte Zeitspanne betrachtet wird und die Parameter des Gleichgewichtsterms festgehalten werden.

Der Grund dafür, alle Parameter für mehrere Experimente gleichzeitig zu identifizieren, ergibt sich aus verschiedenen Tatsachen. Erstens kann eine unzulängliche Menge an experimentellen Daten instabile oder uneindeutige Parameterschätzungen hervorrufen. Wenn die experimentellen Daten unvollständig sind, können diese nicht die gesamte Bandbreite an vorgesehenen Modellfunktionen abdecken (siehe Mahnken [40]). Zweitens soll der Identifikationsprozess die Parameter bezüglich verschiedener Fehlereinflüsse mitteln. Die Parameteridentifikation ist ein Optimierungsproblem, bei dem die Unterschiede zwischen experimentellen und simulierten Daten in der Regel nicht vollständig verschwinden. Diese Tatsache hat zwei verschiedene Hauptursachen: Einerseits kann das gewählte Materialmodell in der Regel nicht allumfassend das mechanische Verhalten eines Materials beschreiben, somit muß der Modellfehler berücksichtigt werden (siehe Mahnken [40]). Auf der anderen Seite treten Ungewißheiten bei experimentellen Versuchen auf. Das bedeutet, dass - wie oben bereits erwähnt - zusätzlich zu möglichen Fehlern in den Messungen ebenfalls Streuungen von Meßdaten aufgrund unterschiedlicher Materialbeschaffenheiten der Probekörper auftreten können. Deshalb wird mit der gleichzeitigen Identifikation aller Parameter ein optimaler Materialparametersatz im Sinne einer optimalen Anpassung des konstitutiven Gesetzes - welches selber einem Modellfehler unterliegt - an alle Experimente erreicht. Darüber hinaus führt die Berücksichtigung aller Experimente innerhalb einer einzigen Optimierungsroutine zu einer verbesserten Anpassung der Materialsimulation an die Streuungen von experimentellen Daten.

Für die repräsentativen Simulationen wird das viskoelastische Materialgesetz, welches von Reese und Govindjee [65] mit mehreren Relaxationstermen vorgeschlagen wurde (siehe Govindjee und Reese [18]), mit Hilfe der Finite-Elemente Methode (FEM) eingesetzt. Unter Befolgung des Ansatzes von Mahnken und Stein ( [48], [47], [49], und Mahnken [40], [42]), wird eine Sensitivitätsanalyse durchgeführt, welche die Inhomogenität der Verschiebungsfelder berücksichtigt. Die Verschiebungsfelder der Proben in den Experimenten werden mit Hilfe berührungsloser optischer Meßverfahren bestimmt. Die Simulation der Experimente ist kraftgesteuert mit den in den Experimenten gemessenen Kraft-Zeit Kurven. 
Die vorliegende Arbeit ist wie folgt gegliedert: In Kapitel 2 werden die grundlegenden Gleichungen für die Beschreibung finiter Hyperelastizität bei Isotropie mit Hilfe nichtlinearer Kontinuumsmechanik aufgeführt. Nach der Betrachtung isotroper Hyperelastizität in Eigenrichtungen werden Verzerrungsenergiefunktionen vom Ogden-Typ kurz dargestellt.

Die Ogden-Verzerrungsenergiefunktionen werden für die konstitutive Routine für finite Viskoelastizität (siehe Reese und Govindjee [65]) verwendet. Die grundlegenden konstitutiven Gleichungen dieses inelastischen Materialgesetzes werden in Kapitel 3 zusammenfassend dargestellt.

Die Zielsetzung in Kapitel 4 ist, die allgemein angewendeten Methoden für die Durchführung einer Parameteridentifikation zu erläutern. Zuerst wird die Definition eines inversen Problems diskutiert. Anschließend erfolgt die Beschreibung optischer Feldmeßmethoden und die Vorgehensweise bei der Interpolation experimentell gemessener Verschiebungsfelder. Daraufhin werden die in dieser Arbeit verwendenden Optimierungsmethoden erläutert und die allgemein innerhalb einer Parameteridentifikation auftretenden Stabilitätsprobleme angesprochen. Im darauf folgenden Kapitel 5 werden die für eine Sensitivitätsanalyse notwendigen Ableitungen präsentiert.

Um das Stabilitätsverhalten des konstitutiven viskoelastischen Gesetzes innerhalb des Identifikationsalgorithmus' einschätzen zu können, wurden numerische Voruntersuchungen mit Hilfe von Re-Identifikationen vorgenommen, deren Ergebnisse in Kapitel 7 diskutiert werden.

In Kapitel 7.1 folgen numerische Beispiele auf der Basis von realen Meßdaten verschiedener Elastomer-Materialien.

Abschnitt 7.1 zeigt die Parameteridentifikation für einen Polyurethanklebstoff im ausgehärteten Zustand, wobei nur das viskoelastische Kurzzeitverhalten - mit einem Relaxationsmodul innerhalb des Materialmodells - anhand eines Zeitversuches untersucht wird.

In Abschnitt 7.2 wird ein Algorithmus für die Identifikation der Materialparameter des viskoelastischen Materialgesetzes präsentiert, in dem die Meßdaten von drei verschiedenen Experimenten eingehen. Das Material ist ein Polyurethanschaum, der in Druckversuchen untersucht wird.

Die Parameteridentifikation für einen kompakten Polyurethanwerkstoff für mehrere Zeitbereiche wird in Abschnitt 7.3 beschrieben. Die grundlegenden Beobachtungen, die in einem Kurz- und Langzeit-Relaxationsversuch und einem Kurzzeitversuch mit einem Lastzyklus gemacht werden, motivieren die Wahl einer Modellstruktur mit zwei Relaxationstermen für die Simulation. Unter Anwendung der Methode der gleichzeitigen Identifikation für mehrere Versuche können hierbei die experimentellen Lastaufbringungszeiten in Langzeitversuchen zeitlich abgekürzt werden und die Prozedur zur Parameteridentifikation bezieht sich nun auf experimentelle Daten von Kurz- und Langzeitversuchen, ohne dass die Parameter entsprechend dieser Zeitbereiche separiert werden müssen. Anhand einer Verifikation kann gezeigt werden, dass die vorgeschlagene Verfahrensweise eine sehr gute Übereinstimmung zwischen experimentellen und simulierten Verschiebungsdaten für alle betrachteten Experimente liefert. Darüber hinaus sind durch den Einsatz dieser Methode zeitaufwendige Langzeitversuche bis zur völligen Spannungsrelaxation nicht weiter notwendig (siehe auch Kleuter et al. [30]). 
Um die generelle Anwendbarkeit der Identifikationsmethode für mehrere Experimente zu zeigen, wird in Kapitel 8 die Parameteridentifikation für Plastizität bei kleinen Verzerrungen präsentiert. Dabei wird für drei Versuche das selbe Versuchsprogramm an drei Versuchsproben einer Aluminium-Gusslegierung untersucht und die Parametersätze für die jeweiligen individuellen Identifikationen und für die Kombination aller Experimente in einer Identifikationsroutine verglichen. 


\section{Finite deformation hyperelasticity}

In this chapter essential equations for the description of finite deformation hyperelasticity by means of nonlinear continuum mechanics are listed (see also, e.g. Ogden [59], Haupt [21], Holzapfel [23], Betten [8] Bonet and Wood [10] and Marsden and Hughes [51]).

\subsection{Kinematics}

Consider the motion $\boldsymbol{x}=\boldsymbol{\varphi}(\boldsymbol{X}, t)$ of a body in three dimensions so that a continuum point $\boldsymbol{X}$ in the material configuration $\mathcal{B}_{0}$ occupies position $\boldsymbol{x}$ in the spatial configuration $\mathcal{B}_{t}$ at current time $t$. The deformation gradient of the motion is defined by $\boldsymbol{F}=\partial_{X} \boldsymbol{\varphi}$. A strain

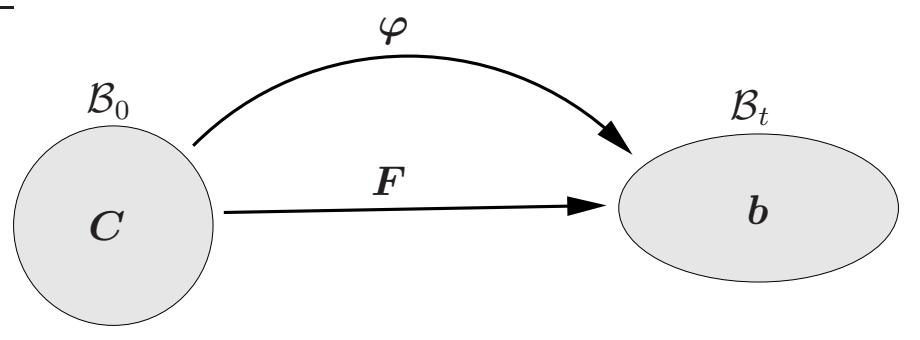

Figure 2.1: Motion $\boldsymbol{x}=\boldsymbol{\varphi}(\boldsymbol{X}, t)$ and corresponding deformation gradient $\boldsymbol{F}=\partial_{\boldsymbol{X}} \boldsymbol{\varphi}$.

measure over the cotangent space to $\mathcal{B}_{0}$ is the right Cauchy-Green tensor $\boldsymbol{C}=\boldsymbol{F}^{\mathrm{T}} \cdot \boldsymbol{g} \cdot \boldsymbol{F}$. Thereby $\boldsymbol{C}=\boldsymbol{\varphi}^{*}(\boldsymbol{g})$ is the pullback of the covariant metric $\boldsymbol{g}$ in the spatial configuration $\mathcal{B}_{t}$. Pushforward operation of the contravariant material metric $\boldsymbol{G}^{-1}$ with $\boldsymbol{b}=\boldsymbol{\varphi}_{*}\left(\boldsymbol{G}^{-1}\right)$ renders the left Cauchy-Green tensor $\boldsymbol{b}=\boldsymbol{F} \cdot \boldsymbol{G}^{-1} \cdot \boldsymbol{F}^{\mathrm{T}}$ which is a strain measure over the tangent space to $\mathcal{B}_{t}$.

\subsubsection{Eigenvalues and eigenvectors of strain tensors}

The polar decomposition of the deformation gradient $\boldsymbol{F}$ into a proper orthogonal rotation tensor $\boldsymbol{R}$ (with $\boldsymbol{R}^{-1}=\boldsymbol{R}^{\mathrm{T}}$, $\operatorname{det} \boldsymbol{R}=1$ ) and the symmetric stretch tensors yields

$$
\boldsymbol{F}=\boldsymbol{R} \cdot \boldsymbol{U}=\boldsymbol{v} \cdot \boldsymbol{R}
$$

Due to the orthogonality of $\boldsymbol{R}$ one obtains for the right Cauchy-Green tensor

$$
\boldsymbol{C}=\boldsymbol{F}^{\mathrm{T}} \cdot \boldsymbol{g} \cdot \boldsymbol{F}=\boldsymbol{U}^{\mathrm{T}} \cdot \boldsymbol{R}^{\mathrm{T}} \cdot \boldsymbol{g} \cdot \boldsymbol{R} \cdot \boldsymbol{U}=\boldsymbol{U}^{\mathrm{T}} \cdot \boldsymbol{g} \cdot \boldsymbol{U}=\boldsymbol{U}^{2} .
$$

The latter result follows from the symmetry of $\boldsymbol{U}$. Analogously the left Cauchy-Green tensor yields

$$
\boldsymbol{b}=\boldsymbol{v}^{2}
$$


The orthogonal and normed set of eigenvectors $\boldsymbol{N}_{\mathrm{a}}$ and its corresponding eigenvalues $\lambda_{\mathrm{a}}$ ( $\mathrm{a}=1,2,3$; no summation) of the material tensor $\boldsymbol{U}$ are introduced with (see Ogden [59])

$$
\boldsymbol{U} \cdot \boldsymbol{N}_{\mathrm{a}}=\lambda_{\mathrm{a}} \boldsymbol{N}_{\mathrm{a}}
$$

where $\left\|\boldsymbol{N}_{\mathrm{a}}\right\|=1$. All eigenvalues $\lambda_{\mathrm{a}}$ are real and positive. The solution of the eigenvalue problem (2.4) delivers the characteristic equation

$$
\lambda^{3}-J_{1} \lambda^{2}+J_{2} \lambda-J_{3}=0,
$$

whose solutions are the eigenvalues $\lambda_{\mathrm{a}}$. The invariants of $\boldsymbol{U}$ are $J_{\mathrm{a}}(\boldsymbol{U})$ which yield

$$
\begin{aligned}
& J_{1}(\boldsymbol{U})=\lambda_{1}+\lambda_{2}+\lambda_{3} \\
& J_{2}(\boldsymbol{U})=\lambda_{1} \lambda_{2}+\lambda_{1} \lambda_{3}+\lambda_{2} \lambda_{3} \\
& J_{3}(\boldsymbol{U})=\lambda_{1} \lambda_{2} \lambda_{3} .
\end{aligned}
$$

By utilizing equation (2.2) with (2.4) the eigenvalue problem for $\boldsymbol{C}$ yields

$$
\boldsymbol{C} \cdot \boldsymbol{N}_{\mathrm{a}}=\boldsymbol{U}^{2} \cdot \boldsymbol{N}_{\mathrm{a}}=\lambda_{\mathrm{a}}^{2} \boldsymbol{N}_{\mathrm{a}} .
$$

The tensors $\boldsymbol{U}$ and $\boldsymbol{C}$ have the same eigenvectors, but different eigenvalues. The eigenvalues of the symmetric tensor $\boldsymbol{U}$ are $\lambda_{\mathrm{a}}$ and are also called principal stretches.

Taking into account the relations $\boldsymbol{R}^{\mathrm{T}} \cdot \boldsymbol{R}=\boldsymbol{I}$ and $\boldsymbol{v}=\boldsymbol{R} \cdot \boldsymbol{U} \cdot \boldsymbol{R}^{\mathrm{T}}$ and equation (2.4) one obtains the eigenvalue problem for $\boldsymbol{v}$

$$
\boldsymbol{v} \cdot\left[\boldsymbol{R} \cdot \boldsymbol{N}_{\mathrm{a}}\right]=\boldsymbol{R} \cdot \boldsymbol{U} \boldsymbol{R}^{\mathrm{T}} \cdot\left[\boldsymbol{R} \cdot \boldsymbol{N}_{\mathrm{a}}\right]=\boldsymbol{R} \cdot \boldsymbol{U} \cdot \boldsymbol{N}_{\mathrm{a}}=\lambda_{\mathrm{a}}\left[\boldsymbol{R} \cdot \boldsymbol{N}_{\mathrm{a}}\right] .
$$

Equations (2.3) and (2.10) lead -in analogy to expression (2.9)- to the eigenvalue problem for $\boldsymbol{b}$

$$
\boldsymbol{b} \cdot\left[\boldsymbol{R} \cdot \boldsymbol{N}_{\mathrm{a}}\right]=\boldsymbol{v}^{2} \cdot\left[\boldsymbol{R} \cdot \boldsymbol{N}_{\mathrm{a}}\right]=\lambda_{\mathrm{a}}^{2}\left[\boldsymbol{R} \cdot \boldsymbol{N}_{\mathrm{a}}\right]
$$

which means that both tensors $\boldsymbol{v}$ and $\boldsymbol{b}$ possess the same eigenvectors $\boldsymbol{R} \cdot \boldsymbol{N}_{\text {a }}$ while the corresponding eigenvalues are $\lambda_{\mathrm{a}}$ and $\lambda_{\mathrm{a}}^{2}$.

The eigenvectors of $\boldsymbol{v}$ and $\boldsymbol{b}$ are the eigenvectors of $\boldsymbol{U}$ and $\boldsymbol{C}$ rotated by $\boldsymbol{R}$. Thus it follows that the eigendirections in the initial configuration $\boldsymbol{N}_{\mathrm{a}}$ are mapped to the spatial eigendirections $\boldsymbol{n}_{\mathrm{a}}$ via

$$
\boldsymbol{n}_{\mathrm{a}}=\boldsymbol{R} \cdot \boldsymbol{N}_{\mathrm{a}}
$$

with $\left\|\boldsymbol{n}_{\mathrm{a}}\right\|=1$. Thus the two-point tensor $\boldsymbol{R}$ rotates the material vectors $\boldsymbol{N}_{\text {a }}$ to the spatial vectors $\boldsymbol{n}_{\mathrm{a}}$.

The introduced symmetric tensors can be represented in the corresponding spectral decompositions by

$$
\begin{aligned}
\boldsymbol{U}^{2} & =\boldsymbol{C}=\sum_{\mathrm{a}=1}^{3} \lambda_{\mathrm{a}}^{2} \boldsymbol{N}_{\mathrm{a}} \otimes \boldsymbol{N}_{\mathrm{a}}, \\
\boldsymbol{v}^{2} & =\boldsymbol{b}=\sum_{\mathrm{a}=1}^{3} \lambda_{\mathrm{a}}^{2} \boldsymbol{n}_{\mathrm{a}} \otimes \boldsymbol{n}_{\mathrm{a}} .
\end{aligned}
$$


Note that both the eigenvalues and eigenvectors are dependent of the position and the time $t$.

In order to describe the two-point tensors $\boldsymbol{F}$ and $\boldsymbol{R}$ in terms of the corresponding eigenvectors and eigenvalues one utilizes the polar decomposition $\boldsymbol{F}=\boldsymbol{R} \cdot \boldsymbol{U}$ and the equations (2.12) and (2.13). Therefore, the deformation gradient yields

$$
\boldsymbol{F}=\boldsymbol{R} \cdot \sum_{\mathrm{a}=1}^{3} \lambda_{\mathrm{a}} \boldsymbol{N}_{\mathrm{a}} \otimes \boldsymbol{N}_{\mathrm{a}}=\sum_{\mathrm{a}=1}^{3} \lambda_{\mathrm{a}}\left[\boldsymbol{R} \cdot \boldsymbol{N}_{\mathrm{a}}\right] \otimes \boldsymbol{N}_{\mathrm{a}}=\sum_{\mathrm{a}=1}^{3} \lambda_{\mathrm{a}} \boldsymbol{n}_{\mathrm{a}} \otimes \boldsymbol{N}_{\mathrm{a}} .
$$

Since the identity tensor $\boldsymbol{I}$ can be expressed as $\boldsymbol{I}=\sum_{\mathrm{a}=1}^{3} \boldsymbol{N}_{\mathrm{a}} \otimes \boldsymbol{N}_{\mathrm{a}}$, together with equation (2.12) it follows that

$$
\boldsymbol{R}=\boldsymbol{R} \cdot \boldsymbol{I}=\sum_{\mathrm{a}=1}^{3}\left[\boldsymbol{R} \cdot \boldsymbol{N}_{\mathrm{a}}\right] \otimes \boldsymbol{N}_{\mathrm{a}}=\sum_{\mathrm{a}=1}^{3} \boldsymbol{n}_{\mathrm{a}} \otimes \boldsymbol{N}_{\mathrm{a}} .
$$

Both tensors are two-point tensors and have eigendirections in the reference configuration $\mathcal{B}_{0}$ and in the spatial configuration $\mathcal{B}_{t}$. Moreover, $\boldsymbol{F}$ and $\boldsymbol{R}$ are not symmetric in general so that the representations (2.15) and (2.16) cannot be considered as spectral representations in the sense of a typical symmetric eigenvalue problem. Thus the eigenstretches $\lambda_{\mathrm{a}}$ in equation (2.15) cannot be interpreted as eigenvalues of the deformation gradient $\boldsymbol{F}$.

\subsection{Isotropic hyperelasticity in terms of invariants}

The requirement for isotropy is that the constitutive behavior has to be identical in every material direction. If the free energy function $\Psi$ as scalar valued tensor function is invariant respecting the superposition of orthogonal transformations it can be expressed via the invariants of its arguments. Therefore, $\Psi$ can be expressed via the invariants of $\boldsymbol{C}$ or $\boldsymbol{b}$ so that

$$
\Psi(\boldsymbol{C})=\Psi\left(I_{1}(\boldsymbol{C}), I_{2}(\boldsymbol{C}), I_{3}(\boldsymbol{C})\right)=\Psi\left(I_{1}(\boldsymbol{b}), I_{2}(\boldsymbol{b}), I_{3}(\boldsymbol{b})\right),
$$

whereby the invariants of $\boldsymbol{C}$ and accordingly $\boldsymbol{b}$ base on the associated eigenvalue problem. Since $\boldsymbol{C}$ and $\boldsymbol{b}$ have the same eigenvalues, for the invariants $I_{\mathrm{a}}=I_{\mathrm{a}}(\boldsymbol{C})$ and $I_{\mathrm{a}}=I_{\mathrm{a}}(\boldsymbol{b})$ $(\mathrm{a}=1,2,3)$ it holds

$$
I_{\mathrm{a}}(\boldsymbol{C})=\left[\boldsymbol{C}: \boldsymbol{G}^{-1}\right]^{\mathrm{a}}=I_{\mathrm{a}}(\boldsymbol{b})=[\boldsymbol{g}: \boldsymbol{b}]^{\mathrm{a}} .
$$

Due to the isotropic restriction the second Piola-Kirchhoff stresses result in

$$
\boldsymbol{S}=2 \frac{\partial \Psi}{\partial \boldsymbol{C}}=2 \partial_{I_{\mathrm{a}}} \Psi \partial_{\boldsymbol{C}} I_{\mathrm{a}}
$$

For the Kirchhoff stresses this leads to

$$
\boldsymbol{\tau}=\boldsymbol{F} \cdot \boldsymbol{S} \cdot \boldsymbol{F}^{\mathrm{T}}=2 \frac{\partial \Psi}{\partial \boldsymbol{g}}=2 \partial_{I_{\mathrm{a}}} \Psi \partial_{\boldsymbol{g}} I_{\mathrm{a}},
$$

and with the partial derivatives of the invariants one obtains

$$
\boldsymbol{\tau}=2\left[\frac{\partial \Psi}{\partial I_{1}} \boldsymbol{b}+2 \frac{\partial \Psi}{\partial I_{2}} \boldsymbol{b} \cdot \boldsymbol{g} \cdot \boldsymbol{b}+3 \frac{\partial \Psi}{\partial I_{3}} \boldsymbol{b} \cdot \boldsymbol{g} \cdot \boldsymbol{b} \cdot \boldsymbol{g} \cdot \boldsymbol{b}\right] .
$$


Now the following relation can be seen: If the partial derivatives of the first invariant $I_{1}$ with respect to $\boldsymbol{g}$ and $\boldsymbol{b}$ are taken

$$
\frac{\partial(\boldsymbol{g}: \boldsymbol{b})}{\partial \boldsymbol{g}}=\boldsymbol{b}, \quad \frac{\partial(\boldsymbol{g}: \boldsymbol{b})}{\partial \boldsymbol{b}}=\boldsymbol{g}
$$

then it follows that (equivalent computations for the partial derivatives of the invariants $I_{2}$ and $I_{3}$ can be made)

$$
\underbrace{\frac{\partial(\boldsymbol{g}: \boldsymbol{b})}{\partial \boldsymbol{g}}}_{\boldsymbol{b}} \cdot \boldsymbol{g}=\boldsymbol{b} \cdot \underbrace{\frac{\partial(\boldsymbol{g}: \boldsymbol{b})}{\partial \boldsymbol{b}}}_{\boldsymbol{g}} .
$$

As $\Psi$ only depends on the invariants one obtains

$$
\frac{\partial \Psi}{\partial \boldsymbol{g}} \cdot \boldsymbol{g}=\boldsymbol{b} \cdot \frac{\partial \Psi}{\partial \boldsymbol{b}}
$$

and due to the symmetry of $\boldsymbol{g}$ and $\boldsymbol{b}$

$$
\boldsymbol{g} \cdot \frac{\partial \Psi}{\partial \boldsymbol{g}}=\frac{\partial \Psi}{\partial \boldsymbol{b}} \cdot \boldsymbol{b}
$$

Hence for the Kirchhoff stresses it follows

$$
\boldsymbol{\tau}=2 \frac{\partial \Psi}{\partial \boldsymbol{g}}=2 \boldsymbol{g}^{-1} \cdot \frac{\partial \Psi}{\partial \boldsymbol{b}} \cdot \boldsymbol{b}
$$

This constitutive equation is an important relation in the theory of isotropic finite hyperelasticity.

\subsection{Isotropic hyperelasticity in terms of principle stretches}

As already mentioned the free energy function of an isotropic material only depends on the invariants of the right (or left) Cauchy-Green tensor. Accordingly, the free energy function can be formulated as a function of the principal stretches (see Ogden [59]). Often this kind of formulation is computationally more convenient. This also holds for the Ogden materials which proved to be very suitable for the modeling of the mechanical behavior of, e.g. rubberlike materials.

\subsubsection{Constitutive equations in terms of the principal stretches}

If the free energy function $\Psi$ is an isotropic tensor function which is invariant to superimposed invertible mappings, it is possible to consider $\Psi$ as a function of eigenvalues (see Ogden [59]). Therefore $\Psi$ can be expressed via

$$
\Psi=\Psi\left(\boldsymbol{C}, \boldsymbol{G}^{-1}\right)=\Psi\left(\lambda_{1}, \lambda_{2}, \lambda_{3}\right) .
$$


For the stress free reference configuration it should hold that $\Psi(1,1,1)=0$. The second Piola-Kirchhoff stresses are calculated by

$$
\boldsymbol{S}=2 \frac{\partial \Psi\left(\lambda_{\mathrm{a}}\right)}{\partial \boldsymbol{C}}=2 \sum_{\mathrm{a}=1}^{3} \frac{\partial \Psi}{\partial \lambda_{\mathrm{a}}} \frac{\partial \lambda_{\mathrm{a}}}{\partial \boldsymbol{C}}
$$

If $\Psi$ is a function of the principal stretches, the derivative $\partial \Psi / \partial \lambda_{\mathrm{a}}$ can be computed directly. The partial derivative $\partial \lambda_{\mathrm{a}} / \partial \boldsymbol{C}$ appearing in the chain rule in equation (2.28) can be determined with equation (2.13) so that

$$
\frac{\partial \lambda_{\mathrm{a}}}{\partial \boldsymbol{C}}=\frac{\partial \lambda_{\mathrm{a}}}{\partial \lambda_{\mathrm{a}}^{2}} \frac{\partial \lambda_{\mathrm{a}}^{2}}{\partial \boldsymbol{C}}=\frac{1}{2 \lambda_{\mathrm{a}}} \boldsymbol{N}_{\mathrm{a}} \otimes \boldsymbol{N}_{\mathrm{a}}
$$

By this one obtains for the second Piola-Kirchhoff stresses

$$
\boldsymbol{S}=\sum_{\mathrm{a}=1}^{3} \frac{1}{\lambda_{\mathrm{a}}} \frac{\partial \Psi}{\partial \lambda_{\mathrm{a}}} \boldsymbol{N}_{\mathrm{a}} \otimes \boldsymbol{N}_{\mathrm{a}}=\sum_{\mathrm{a}=1}^{3} S_{\mathrm{a}} \boldsymbol{N}_{\mathrm{a}} \otimes \boldsymbol{N}_{\mathrm{a}}
$$

The comparison with the spectral decomposition of the right Cauchy-Green tensor (see equation (2.13)) shows that $\boldsymbol{S}$ and $\boldsymbol{C}$ possess the same eigenvectors which is a consequence of the restriction to isotropic material behavior. Since the eigenvectors in the spatial configuration are computed by pure rotation of the eigenvectors $\boldsymbol{N}_{\mathrm{a}}$ with $\boldsymbol{n}_{\mathrm{a}}=\boldsymbol{R} \cdot \boldsymbol{N}_{\mathrm{a}}=$ $\lambda_{\mathrm{a}}^{-1} \boldsymbol{F} \cdot \boldsymbol{N}_{\mathrm{a}}$, for the Kirchhoff stress tensor it follows with $\boldsymbol{\tau}=\boldsymbol{F} \cdot \boldsymbol{S} \cdot \boldsymbol{F}^{\mathrm{T}}$

$$
\boldsymbol{\tau}=\sum_{\mathrm{a}=1}^{3} \lambda_{\mathrm{a}} \frac{\partial \Psi}{\partial \lambda_{\mathrm{a}}} \boldsymbol{n}_{\mathrm{a}} \otimes \boldsymbol{n}_{\mathrm{a}}=\sum_{\mathrm{a}=1}^{3} \tau_{\mathrm{a}} \boldsymbol{n}_{\mathrm{a}} \otimes \boldsymbol{n}_{\mathrm{a}}
$$

which means that for the principal values $\tau_{\mathrm{a}}=\lambda_{\mathrm{a}}^{2} S_{\mathrm{a}}$ holds.

Here it is to be mentioned that a further approach for the determination of equation (2.31) is also possible by the following consideration: Consider the left stretch tensor $\boldsymbol{v}=\boldsymbol{b}^{1 / 2}$ which describes the deformed state of the isotropic hyperelastic material. It is known from the eigenvalue problem (see equation (2.10)) that $\lambda_{\mathrm{a}}$ describe the three real eigenvalues (eigenstretches) of $\boldsymbol{v}$. Since the eigendirections of $\boldsymbol{v}$ coincide with the eigendirections of $\boldsymbol{b}$ they also coincide with the eigendirections of the Kirchhoff stress tensor $\boldsymbol{\tau}$ (see representation in equation (2.21)).

\subsubsection{Ogden material model}

This class of Ogden material models (see Ogden [59]) is a generalized free energy function which plays an important role in the theory of finite elasticity. Especially for the description of rubber elasticity these Ogden-type functions are used most extensively due to their good approximation of the experimental observations. 
In this model the postulated free energy is a function of the eigenstretches $\lambda_{\mathrm{a}}$ with

$$
\begin{aligned}
\Psi= & \underbrace{\sum_{\mathrm{T}}^{\mathrm{N}_{\mathrm{T}}} \frac{\mu_{\mathrm{r}}}{\alpha_{\mathrm{r}}}\left[\left[\lambda_{1}^{4 / 3} \lambda_{2}^{-2 / 3} \lambda_{3}^{-2 / 3}\right]^{\alpha_{\mathrm{r}} / 2}+\left[\lambda_{1}^{-2 / 3} \lambda_{2}^{4 / 3} \lambda_{3}^{-2 / 3}\right]^{\alpha_{\mathrm{r}} / 2}+\left[\lambda_{1}^{-2 / 3} \lambda_{2}^{-2 / 3} \lambda_{3}^{4 / 3}\right]^{\alpha_{\mathrm{r}} / 2}-3\right]}_{\Psi_{\mathrm{rev}}^{\mathrm{dev}}} \\
& \quad+\underbrace{\frac{\kappa_{0}}{4}\left[\left[\lambda_{1} \lambda_{2} \lambda_{3}\right]^{2}-2 \ln \left[\lambda_{1} \lambda_{2} \lambda_{3}\right]-1\right]}_{\Psi^{\mathrm{vol}}} \\
= & \Psi^{\mathrm{dev}}+\Psi^{\mathrm{vol}} .
\end{aligned}
$$

The positive integer $\mathrm{N}_{\mathrm{T}}$ describes the number of terms in the isochoric part of $\Psi$. Moreover, $\mu_{\mathrm{r}}$ are shear moduli and $\alpha_{\mathrm{r}}$ are dimensionless constants for $\mathrm{r}=1, \ldots, \mathrm{N}_{\mathrm{T}}$. In the volumetric part of $\Psi$ the compression modulus is described by $\kappa_{0}$.

For the later computations considering nonlinear viscoelasticity the free energy function is described as a function of the eigenvalues $b_{a}=\lambda_{a}^{2}$ of the left Cauchy-Green tensor (see equation (2.14)). With an additive split of $\Psi$ into parts which contain the isochoric and volumetric strain measures separately one obtains

$$
\Psi=\Psi^{\operatorname{dev}}\left(\overline{\mathrm{b}}_{1}, \overline{\mathrm{b}}_{2}, \overline{\mathrm{b}}_{3}\right)+\Psi^{\mathrm{vol}}(J) .
$$

The determinant of $\boldsymbol{F}$ is referred to as

$$
J=\lambda_{1} \lambda_{2} \lambda_{3}
$$

Consequently, the eigenvalues of the isochoric left Cauchy-Green tensor yield ${ }^{1}$

$$
\overline{\mathrm{b}}_{\mathrm{a}}=J^{-2 / 3} \mathrm{~b}_{\mathrm{a}} .
$$

Finally the free energy function can be expressed as

$$
\Psi=\underbrace{\sum_{\mathrm{r}=1}^{N_{\mathrm{T}}} \frac{\mu_{\mathrm{r}}}{\alpha_{\mathrm{r}}}\left[\overline{\mathrm{b}}_{1}^{\alpha_{\mathrm{r}} / 2}+\overline{\mathrm{b}}_{2}^{\alpha_{\mathrm{r}} / 2}+\overline{\mathrm{b}}_{3}^{\alpha_{\mathrm{r}} / 2}-3\right]}_{\Psi^{\text {dev }}}+\underbrace{\frac{\kappa_{0}}{4}\left[J^{2}-2 \ln J-1\right]}_{\Psi^{\text {vol }}} .
$$

\footnotetext{
${ }^{1} \mathrm{~A}$ volumetric/isochoric multiplicative decomposition of the deformation gradient $\boldsymbol{F}$ yields

$$
\boldsymbol{F}=\overline{\boldsymbol{F}} \cdot \boldsymbol{F}^{\mathrm{vol}},
$$

with $\boldsymbol{F}^{\mathrm{vol}}=J^{1 / 3} \boldsymbol{I}$ and $\overline{\boldsymbol{F}}=J^{-1 / 3} \boldsymbol{F}$. Hence the isochoric left Cauchy-Green tensor yields

$$
\begin{aligned}
\overline{\boldsymbol{b}} & =\overline{\boldsymbol{F}} \cdot \boldsymbol{G}^{-1} \cdot \overline{\boldsymbol{F}}^{\mathrm{T}}=J^{-2 / 3} \boldsymbol{F} \cdot \boldsymbol{G}^{-1} \cdot \boldsymbol{F}^{\mathrm{T}} \\
& =J^{-2 / 3} \boldsymbol{b} \\
& =J^{-2 / 3} \sum_{\mathrm{a}=1}^{3} \lambda_{\mathrm{a}}^{2} \boldsymbol{n}_{\mathrm{a}} \otimes \boldsymbol{n}_{\mathrm{a}}=J^{-2 / 3} \sum_{\mathrm{a}=1}^{3} \mathrm{~b}_{\mathrm{a}} \boldsymbol{n}_{\mathrm{a}} \otimes \boldsymbol{n}_{\mathrm{a}} \\
& =\sum_{\mathrm{a}=1}^{3}\left[\lambda_{1} \lambda_{2} \lambda_{3}\right]^{-2 / 3} \lambda_{\mathrm{a}}^{2} \boldsymbol{n}_{\mathrm{a}} \otimes \boldsymbol{n}_{\mathrm{a}} \\
& =\sum_{\mathrm{a}=1}^{3} \overline{\mathrm{b}}_{\mathrm{a}} \boldsymbol{n}_{\mathrm{a}} \otimes \boldsymbol{n}_{\mathrm{a}} .
\end{aligned}
$$
}


Here for the isochoric part of the free energy the notation $\Psi^{\mathrm{dev}}$ is applied, since for isotropy the deviatoric Kirchhoff stresses are computed by this part. The deviatoric and volumetric part of the principal Kirchhoff stresses take the form (see equation 2.31)

$$
\tau_{\mathrm{a}}=\underbrace{\sum_{\mathrm{b}=1}^{3} \frac{\partial \Psi^{\mathrm{dev}}}{\partial \overline{\mathrm{b}}_{b}} \frac{\partial \overline{\mathrm{b}}_{b}}{\partial \lambda_{\mathrm{a}}} \lambda_{\mathrm{a}}}_{\left[\tau_{\mathrm{a}}\right]^{\operatorname{dev}}}+\underbrace{\frac{\partial \Psi^{\mathrm{vol}}}{\partial J} J}_{\frac{1}{3} \boldsymbol{\tau}: \boldsymbol{g}}
$$

with

$$
\frac{\partial \Psi^{\mathrm{dev}}}{\partial \overline{\mathrm{b}}_{b}}=\sum_{\mathrm{r}=1}^{\mathrm{N}_{\mathrm{T}}} \frac{\mu_{\mathrm{r}}}{2} \overline{\mathrm{b}}_{\mathrm{b}}^{\left.\left[\alpha_{\mathrm{r}} / 2\right]-1\right]}, \quad \frac{\partial \Psi^{\mathrm{vol}}}{\partial J}=\frac{\kappa_{0}}{2}\left[J-J^{-1}\right]
$$

and

$$
\frac{\partial \overline{\mathrm{b}}_{a}}{\partial \lambda_{\mathrm{a}}}=\frac{4}{3} J^{-[2 / 3]} \lambda_{\mathrm{a}}
$$

and for $\mathrm{a} \neq \mathrm{b}$

$$
\frac{\partial \overline{\mathrm{b}}_{b}}{\partial \lambda_{\mathrm{a}}}=-\frac{2}{3} J^{-[2 / 3]} \lambda_{\mathrm{b}}^{2} \lambda_{\mathrm{a}}^{-1}
$$

which yields for $\mathrm{a} \neq \mathrm{b}, \mathrm{a} \neq \mathrm{c}, \mathrm{b} \neq \mathrm{c}$ :

$$
\begin{aligned}
\tau_{\mathrm{a}}= & \sum_{\mathrm{r}=1}^{\mathrm{N}_{\mathrm{T}}} \frac{\mu_{\mathrm{r}}}{2}[\overline{\mathrm{b}}_{\mathrm{a}}^{\left[\left[\alpha_{\mathrm{r}} / 2\right]-1\right]} \frac{4}{3} \underbrace{J^{-[2 / 3]} \lambda_{\mathrm{a}}^{2}}_{\overline{\mathrm{b}}_{\mathrm{a}}}-\overline{\mathrm{b}}_{\mathrm{b}}^{\left[\left[\alpha_{\mathrm{r}} / 2\right]-1\right]} \frac{2}{3} \underbrace{J^{-[2 / 3]} \lambda_{\mathrm{b}}^{2}}_{\overline{\mathrm{b}}_{\mathrm{b}}} \\
& \quad-\overline{\mathrm{b}}_{\mathrm{c}}^{\left[\left[\alpha_{\mathrm{r}} / 2\right]-1\right]} \frac{2}{3} \underbrace{J^{-[2 / 3]} \lambda_{\mathrm{c}}^{2}}_{\overline{\mathrm{b}}_{\mathrm{c}}}]+\frac{\kappa_{0}}{2}\left[J^{2}-1\right] \\
= & \underbrace{\sum_{\mathrm{r}=1}^{N_{\mathrm{T}}} \mu_{\mathrm{r}}\left[\frac{2}{3} \overline{\mathrm{b}}_{\mathrm{a}}^{\alpha_{\mathrm{r}} / 2}-\frac{1}{3} \overline{\mathrm{b}}_{\mathrm{b}}^{\alpha_{\mathrm{r}} / 2}-\frac{1}{3} \overline{\mathrm{b}}_{\mathrm{c}}^{\alpha_{\mathrm{r}} / 2}\right]}_{\left[\tau_{\mathrm{a}}\right] \operatorname{dev}}+\underbrace{\frac{\kappa_{0}}{2}\left[J^{2}-1\right]}_{\frac{1}{3} \boldsymbol{\tau}: \boldsymbol{g}} .
\end{aligned}
$$




\section{Finite deformation viscoelasticity in principal directions}

In order to establish a simulation tool for the precise prediction and the appropriate design of elastomer components, a suitable constitutive law - being able to simulate all characteristic material behaviors like relaxation, creep, frequency-dependent stiffness and damping properties - is necessary.

The stress-strain response of most elastomers can be described by two different characteristics. First the Mullins effect, a stress softening occurring in the first load cycle(s) and second a viscoelastic material behavior.

Detailed descriptions of the classical theory of rate dependent materials can be found for instance in Findley [17], Eringen [16], Lemaitre and Chaboche [32], Haupt [21], Holzapfel [23], Kaliske and Rothert [27] as well as Simo and Hughes [70].

Constitutive laws which describe the stress softening due to the Mullins effect have been elaborated by, e.g. Govindjee and Simo [19], Böl [9] and Ogden and Roxburgh [60].

Considering pre-damaged filled elastomers, which are in a stable stationary state respecting the Mullins effect, for this work a viscoelastic material model at large strains is chosen. In the material theories of Simo [68], Holzapfel and Simo [24] and Simo and Hughes [70] the evolution laws are expressed as phenomenological linear differential equations of overstress. In 1998 Reese and Govindjee [65] proposed a continuum formulation which is in contrast to many other theories not restricted to viscoelastic response for strain rates near the thermodynamic equilibrium, see also Govindjee and Reese [18], Reese and Govindjee [64], Lion [34], [35], Lundin and Klarbring [39], Haupt [21], Holzapfel [23] and also Huber and Tsakmakis [25].

In this chapter the theory of the viscoelastic material model proposed by Reese and Govindjee [65] is reviewed and the numerical implementation is discussed. This compressible viscoelasticity law is formulated for isotropy within the theory of finite deformations. Considering multiple relaxation mechanisms a set of internal variables based on the multiplicative split of the deformation gradient (see also Lubliner [37]) into elastic and viscous parts is introduced. Each relaxation mechanism can be represented by a viscous damper and an elastic spring connected consecutively. The strains in the dampers and by this the inelastic and accordingly viscous parts of the deformation gradient yield the internal variables for which nonlinear rate dependent evolution equations are introduced. The derivation of the model is made so that the second law of thermodynamics - given by the Clausius-Duhem inequality - is satisfied for every admissible process. A flow rule for the viscous strain rate, which is valid far from thermodynamic equilibrium, is assumed to apply. Using logarithmic strain measures, the integration for the viscous evolution law is made using an exponential time integration method (see also Weber and Anand [73]). 


\subsection{Kinematics of finite viscoelasticity}

For the case of several relaxation mechanisms $(\mathrm{k}=1, \ldots, \mathrm{N})$ the deformation gradient

$$
\boldsymbol{F}=\boldsymbol{F}_{\mathrm{e}}^{\mathrm{k}} \cdot \boldsymbol{F}_{\mathrm{v}}^{\mathrm{k}}
$$

(Reese and Govindjee [65]) is split into elastic and non-reversible viscous parts by multiplicative decompositions. Each relaxation mechanism can be represented by a viscous damper and an elastic spring connected consecutively. For the following theory this basic assumption gives the kinematical foundation. As illustrated in figure 3.1 for each viscous damper separately $\mathcal{B}_{v}^{\mathrm{k}}$ is viewed as defining a local stress-free intermediate configuration whereby $\boldsymbol{F}_{\mathrm{v}}^{\mathrm{k}}$ is the associated internal variable defining its current state. The following

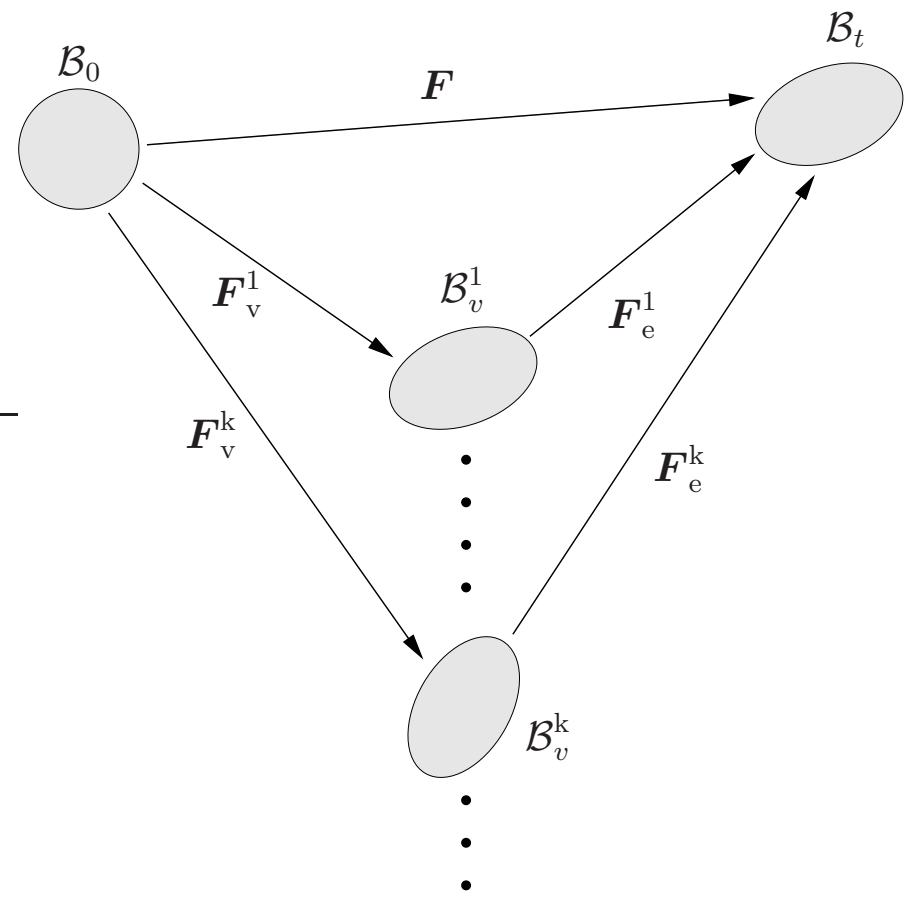

Figure 3.1: Multiplicative decomposition.

strain measures - being associated with the multiplicative decomposition - are introduced. The elastic right Cauchy-Green tensor $\boldsymbol{C}_{\mathrm{e}}^{\mathrm{k}}$ in the intermediate configuration $\mathcal{B}_{v}^{\mathrm{k}}$ is the pullback of the spatial covariant metric $\boldsymbol{g}$ and accordingly the pushforward of the right Cauchy-Green tensor $\boldsymbol{C}$ from $\mathcal{B}_{0}$ to $\mathcal{B}_{v}^{\mathrm{k}}$ with

$$
\boldsymbol{C}_{\mathrm{e}}^{\mathrm{k}}=\left[\boldsymbol{F}_{\mathrm{e}}^{\mathrm{k}}\right]^{\mathrm{T}} \cdot \boldsymbol{g} \cdot \boldsymbol{F}_{\mathrm{e}}^{\mathrm{k}}=\left[\boldsymbol{F}_{\mathrm{v}}^{\mathrm{k}}\right]^{-\mathrm{T}} \cdot \boldsymbol{C} \cdot\left[\boldsymbol{F}_{\mathrm{v}}^{\mathrm{k}}\right]^{-1} \text {. }
$$

The spatial elastic left Cauchy-Green tensor $\boldsymbol{b}_{\mathrm{e}}^{\mathrm{k}}$ is computed by pushforward operation of the contravariant metric $\boldsymbol{G}_{\mathrm{v}}^{-1}$ from $\mathcal{B}_{v}^{\mathrm{k}}$ to $\mathcal{B}_{t}$ or by pushforward operation of the inverse viscous right Cauchy-Green tensor $\left[\boldsymbol{C}_{\mathrm{v}}^{\mathrm{k}}\right]^{-1}$ from $\mathcal{B}_{0}$ to $\mathcal{B}_{t}$ with

$$
\boldsymbol{b}_{\mathrm{e}}^{\mathrm{k}}=\boldsymbol{F}_{\mathrm{e}}^{\mathrm{k}} \cdot \boldsymbol{G}_{\mathrm{v}}^{-1} \cdot\left[\boldsymbol{F}_{\mathrm{e}}^{\mathrm{k}}\right]^{\mathrm{T}}=\boldsymbol{F} \cdot\left[\boldsymbol{C}_{\mathrm{v}}^{\mathrm{k}}\right]^{-1} \cdot \boldsymbol{F}^{\mathrm{T}} .
$$




\subsubsection{Time derivatives}

The expression $\mathrm{D}_{t}\{\bullet\}=\left.\partial_{t}\{\bullet\}\right|_{\boldsymbol{X}}$ defines the material time derivative $\mathrm{D}_{t}$ of an arbitrary quantity $\{\bullet\}$ at fixed material placement $\boldsymbol{X}$. The material time derivative of the motion map $\boldsymbol{\varphi}$ yields the spatial velocity $\boldsymbol{v}$ with

$$
\boldsymbol{v}=\mathrm{D}_{t} \boldsymbol{\varphi}(\boldsymbol{X}, t) .
$$

Therefore, the material gradient of $\boldsymbol{v}$ yields the material time derivative of the spatial deformation gradient $\boldsymbol{F}$ with

$$
\mathrm{D}_{t} \boldsymbol{F}=\partial_{\boldsymbol{X}} \boldsymbol{v}
$$

and the spatial gradient of $\boldsymbol{v}$ is denoted as the spatial velocity gradient $\boldsymbol{l}$ with

$$
\boldsymbol{l}=\partial_{\boldsymbol{x}} \boldsymbol{v}=\mathrm{D}_{t} \boldsymbol{F} \cdot \boldsymbol{F}^{-1} .
$$

The material time derivative of spatial quantities is not objective in general. However, the principle of frame indifference requires constitutive equations to be invariant under superposition of spatial rigid body motions. This motivates the introduction of an objective time derivative - the Lie derivative - which is the material time derivative of a spatial tensor field considering the deformed basis as being constant. Considering a spatial tensor field $\boldsymbol{f}$ the Lie derivative $\mathrm{L}_{t} \boldsymbol{f}$ is defined as follows

$$
\mathrm{L}_{t}=\boldsymbol{\varphi}_{*}\left(\mathrm{D}_{t}\left(\boldsymbol{\varphi}^{*}(\boldsymbol{f})\right)\right) .
$$

Thereby, first the pull-back operation $\varphi^{*}(\boldsymbol{f})$ of $\boldsymbol{f}$ to the reference configuration is computed. Then the result of the material time derivative $\mathrm{D}_{t}\left(\boldsymbol{\varphi}^{*}(\boldsymbol{f})\right)$ is pushed forward to the spatial configuration.

\subsection{Constitutive model}

In this section the constitutive equations are reviewed for the general case of multiple relaxation mechanisms (see Govindjee and Reese [65]).

\subsubsection{Free energy function}

It is assumed that the free energy for the nonlinear viscoelastic material model is a function of $\boldsymbol{C}$ and $\boldsymbol{C}_{\mathrm{e}}^{\mathrm{k}}$ as follows

$$
\Psi=\Psi\left(\boldsymbol{C}, \boldsymbol{F}_{\mathrm{v}}^{\mathrm{k}}\right)=\Psi^{\mathrm{eq}}(\boldsymbol{C})+\sum_{\mathrm{k}=1}^{\mathrm{N}} \Psi^{\mathrm{k}}\left(\boldsymbol{C}_{\mathrm{e}}^{\mathrm{k}}=\left[\boldsymbol{F}_{\mathrm{v}}^{\mathrm{k}}\right]^{-\mathrm{T}} \cdot \boldsymbol{C} \cdot\left[\boldsymbol{F}_{\mathrm{v}}^{\mathrm{k}}\right]^{-1}\right) .
$$

Considering a rheological model consisting of $N$ Maxwell elements in parallel with an auxiliary spring, the equilibrium part $\Psi^{\mathrm{eq}}(\boldsymbol{C})$ of the free energy is computed by the strain in the auxiliary spring whereas the $\mathrm{k}$ non-equilibrium parts of the free energy depend on the strain in the spring of the corresponding Maxwell element $\mathrm{k}$. Moreover, for $\Psi^{\mathrm{eq}}$ and $\Psi^{\mathrm{k}}$ it should hold that if $\boldsymbol{F}$ and $\boldsymbol{F}_{\mathrm{e}}$ and $\boldsymbol{F}_{\mathrm{v}}^{\mathrm{k}}$ are the identity tensors, the tensors $\boldsymbol{S}$ and $\boldsymbol{S}_{\text {eq }}$ and $\boldsymbol{S}^{\mathrm{k}}$ vanish and also $\Psi=0$ and $\Psi^{\mathrm{eq}}=0$ and $\Psi^{\mathrm{k}}=0$ hold, respectively. 


\subsubsection{Notes on thermodynamics}

The isothermal Clausius-Duhem inequality is given by

$$
\mathcal{D}=\frac{1}{2} \boldsymbol{S}: \mathrm{D}_{t} \boldsymbol{C}-\mathrm{D}_{t} \Psi \geq 0
$$

whereby $\mathcal{D}$ is the difference between the stress power $\mathcal{P}_{\text {int }}=\frac{1}{2} \boldsymbol{S}: \mathrm{D}_{t} \boldsymbol{C}$ and the rate of change in free energy $\mathrm{D}_{t} \Psi$. Substituting the material time derivative of equation (3.8) into the inequality equation (3.9) yields

$$
\begin{aligned}
\mathcal{D} & =\frac{1}{2} \boldsymbol{S}: \mathrm{D}_{t} \boldsymbol{C}-\mathrm{D}_{t} \Psi \\
& =\frac{1}{2} \boldsymbol{S}: \mathrm{D}_{t} \boldsymbol{C}-\frac{\partial \Psi}{\partial \boldsymbol{C}}: \mathrm{D}_{t} \boldsymbol{C}-\sum_{\mathrm{k}=1}^{\mathrm{N}} \frac{\partial \Psi}{\partial \boldsymbol{F}_{\mathrm{v}}^{\mathrm{k}}}: \mathrm{D}_{t} \boldsymbol{F}_{\mathrm{v}}^{\mathrm{k}} \\
& =\left[\frac{1}{2} \boldsymbol{S}-\frac{\partial \Psi^{\mathrm{eq}}}{\partial \boldsymbol{C}}-\sum_{\mathrm{k}=1}^{\mathrm{N}} \frac{\partial \Psi^{\mathrm{k}}}{\partial \boldsymbol{C}}\right]: \mathrm{D}_{t} \boldsymbol{C}-\sum_{\mathrm{k}=1}^{\mathrm{N}} \frac{\partial \Psi^{\mathrm{k}}}{\partial \boldsymbol{F}_{\mathrm{v}}^{\mathrm{k}}}: \mathrm{D}_{t} \boldsymbol{F}_{\mathrm{v}}^{\mathrm{k}} \geq 0 .
\end{aligned}
$$

A standard argument of Coleman and Noll then yields the state equation defining the elastic law as follows

$$
\frac{1}{2} \boldsymbol{S}-\frac{\partial \Psi^{\mathrm{eq}}}{\partial \boldsymbol{C}}-\sum_{\mathrm{k}=1}^{\mathrm{N}} \frac{\partial \Psi^{\mathrm{k}}}{\partial \boldsymbol{C}}=\mathbf{0} .
$$

Thus the second Piola-Kirchhoff stresses result in

$$
\boldsymbol{S}=2 \frac{\partial \Psi}{\partial \boldsymbol{C}}=\boldsymbol{S}^{\mathrm{eq}}+\sum_{\mathrm{k}=1}^{\mathrm{N}} \boldsymbol{S}^{\mathrm{k}}
$$

with the equilibrium part and the non-equilibrium parts

$$
\boldsymbol{S}^{\mathrm{eq}}=2 \frac{\partial \Psi^{\mathrm{eq}}}{\partial \boldsymbol{C}}, \quad \boldsymbol{S}^{\mathrm{k}}=2 \frac{\partial \Psi^{\mathrm{k}}}{\partial \boldsymbol{C}}
$$

By transforming the non-equilibrium parts $\boldsymbol{S}^{\mathrm{k}}$ into functions depending on the variables $\boldsymbol{F}_{\mathrm{e}}^{\mathrm{k}}$ one obtains

$$
\begin{aligned}
\boldsymbol{S}^{\mathrm{k}} & =2 \frac{\partial \Psi^{\mathrm{k}}}{\partial \boldsymbol{C}} \\
& =2 \frac{\partial \Psi^{\mathrm{k}}}{\partial \boldsymbol{C}_{\mathrm{e}}^{\mathrm{k}}}: \frac{\partial \boldsymbol{C}_{\mathrm{e}}^{\mathrm{k}}}{\partial \boldsymbol{C}} \\
& =2 \frac{\partial \Psi^{\mathrm{k}}}{\partial \boldsymbol{C}_{\mathrm{e}}^{\mathrm{k}}}: \frac{1}{2}\left(\left[\boldsymbol{F}_{\mathrm{v}}^{\mathrm{k}}\right]^{-\mathrm{T}} \bar{\otimes}\left[\boldsymbol{F}_{\mathrm{v}}^{\mathrm{k}}\right]^{-T}+\left[\boldsymbol{F}_{\mathrm{v}}^{\mathrm{k}}\right]^{-\mathrm{T}} \underline{\otimes}\left[\boldsymbol{F}_{\mathrm{v}}^{\mathrm{k}}\right]^{-T}\right) \\
& =2\left[\boldsymbol{F}_{\mathrm{v}}^{\mathrm{k}}\right]^{-1} \cdot \frac{\partial \Psi^{\mathrm{k}}}{\partial \boldsymbol{C}_{\mathrm{e}}^{\mathrm{k}}} \cdot\left[\boldsymbol{F}_{\mathrm{v}}^{\mathrm{k}}\right]^{-\mathrm{T}}
\end{aligned}
$$

with

$$
\begin{aligned}
& {\left[\left[\boldsymbol{F}_{\mathrm{v}}^{\mathrm{k}}\right]^{-\mathrm{T}} \bar{\otimes}\left[\boldsymbol{F}_{\mathrm{v}}^{\mathrm{k}}\right]^{-1}+\left[\boldsymbol{F}_{\mathrm{v}}^{\mathrm{k}}\right]^{-\mathrm{T}} \underline{\otimes}\left[\boldsymbol{F}_{\mathrm{v}}^{\mathrm{k}}\right]^{-1}\right]^{I J K L}=} {\left[\left[\boldsymbol{F}_{\mathrm{v}}^{\mathrm{k}}\right]^{-\mathrm{T}}\right]^{I K}\left[\left[\boldsymbol{F}_{\mathrm{v}}^{\mathrm{k}}\right]^{-1}\right]^{J L} } \\
&+\left[\left[\boldsymbol{F}_{\mathrm{v}}^{\mathrm{k}}\right]^{-\mathrm{T}}\right]^{I L}\left[\left[\boldsymbol{F}_{\mathrm{v}}^{\mathrm{k}}\right]^{-1}\right]^{J K} .
\end{aligned}
$$


Therefore, the second Piola-Kirchhoff stresses yield

$$
\begin{aligned}
\boldsymbol{S} & =2 \frac{\partial \Psi}{\partial \boldsymbol{C}} \\
& =2 \frac{\partial \Psi^{\mathrm{eq}}}{\partial \boldsymbol{C}}+\sum_{\mathrm{k}=1}^{\mathrm{N}} 2\left[\boldsymbol{F}_{\mathrm{v}}^{\mathrm{k}}\right]^{-1} \cdot \frac{\partial \Psi^{\mathrm{k}}}{\partial \boldsymbol{C}_{\mathrm{e}}^{\mathrm{k}}} \cdot\left[\boldsymbol{F}_{\mathrm{v}}^{\mathrm{k}}\right]^{-\mathrm{T}} .
\end{aligned}
$$

Applying the standard arguments to equation (3.10) leaves also the reduced dissipation inequality to be satisfied for every individual mechanism so that

$$
\left[\mathcal{D}^{r e d}\right]^{\mathrm{k}}=-\frac{\partial \Psi^{\mathrm{k}}}{\partial \boldsymbol{F}_{\mathrm{v}}^{\mathrm{k}}}: \mathrm{D}_{t} \boldsymbol{F}_{\mathrm{v}}^{\mathrm{k}} \geq 0 .
$$

In order to describe the latter equations in terms of expressions defined on the intermediate configuration the transformation

$$
\begin{aligned}
\frac{\partial \Psi^{\mathrm{k}}}{\partial \boldsymbol{F}_{\mathrm{v}}^{\mathrm{k}}} & =\frac{\partial \Psi^{\mathrm{k}}}{\partial \boldsymbol{C}_{\mathrm{e}}^{\mathrm{k}}}: \frac{\partial \boldsymbol{C}_{\mathrm{e}}^{\mathrm{k}}}{\partial \boldsymbol{F}_{\mathrm{v}}^{\mathrm{k}}} \\
& =-\frac{\partial \Psi^{\mathrm{k}}}{\partial \boldsymbol{C}_{\mathrm{e}}^{\mathrm{k}}}:\left[\boldsymbol{C}_{\mathrm{e}}^{\mathrm{k}} \bar{\otimes}\left[\boldsymbol{F}_{\mathrm{v}}^{\mathrm{k}}\right]^{-\mathrm{T}}+\left[\boldsymbol{F}_{\mathrm{v}}^{\mathrm{k}}\right]^{-\mathrm{T}} \otimes \boldsymbol{C}_{\mathrm{e}}^{\mathrm{k}}\right] \\
& =-2 \boldsymbol{C}_{\mathrm{e}}^{\mathrm{k}} \cdot \frac{\partial \Psi^{\mathrm{k}}}{\partial \boldsymbol{C}_{\mathrm{e}}^{\mathrm{k}}} \cdot\left[\boldsymbol{F}_{\mathrm{v}}^{\mathrm{k}}\right]^{-\mathrm{T}}
\end{aligned}
$$

is considered. Noting that

$$
\overline{\boldsymbol{S}}^{\mathrm{k}}=2 \frac{\partial \Psi^{\mathrm{k}}}{\partial \boldsymbol{C}_{\mathrm{e}}^{\mathrm{k}}}
$$

are second Piola-Kirchhoff stress tensors relative to the intermediate configuration, the modified form of the dissipation inequality becomes

$$
\begin{aligned}
{\left[\mathcal{D}^{\text {red }}\right]^{\mathrm{k}} } & =\left[\boldsymbol{C}_{\mathrm{e}}^{\mathrm{k}} \cdot \overline{\boldsymbol{S}}^{\mathrm{k}} \cdot\left[\boldsymbol{F}_{\mathrm{v}}^{\mathrm{k}}\right]^{-\mathrm{T}}\right]: \mathrm{D}_{t} \boldsymbol{F}_{\mathrm{v}}^{\mathrm{k}} \\
& =\left[\boldsymbol{C}_{\mathrm{e}}^{\mathrm{k}} \cdot \overline{\boldsymbol{S}}^{\mathrm{k}}\right]:\left[\mathrm{D}_{t} \boldsymbol{F}_{\mathrm{v}}^{\mathrm{k}} \cdot\left[\boldsymbol{F}_{\mathrm{v}}^{\mathrm{k}}\right]^{-1}\right] \\
& =\left[\boldsymbol{C}_{\mathrm{e}}^{\mathrm{k}} \cdot \overline{\boldsymbol{S}}^{\mathrm{k}}\right]: \boldsymbol{L}_{\mathrm{v}}^{\mathrm{k}} \geq 0 .
\end{aligned}
$$

Here the viscous velocity gradient in the intermediate configuration is defined as

$$
\boldsymbol{L}_{\mathrm{v}}^{\mathrm{k}}=\mathrm{D}_{t} \boldsymbol{F}_{\mathrm{v}}^{\mathrm{k}} \cdot\left[\boldsymbol{F}_{\mathrm{v}}^{\mathrm{k}}\right]^{-1} .
$$

Since $\overline{\boldsymbol{S}}^{\mathrm{k}}$ and $\boldsymbol{C}_{\mathrm{e}}^{\mathrm{k}}$ commute for isotropy the expression $\boldsymbol{M}^{\mathrm{k}}=\boldsymbol{C}_{\mathrm{e}}^{\mathrm{k}} \cdot \overline{\boldsymbol{S}}^{\mathrm{k}}$ must be symmetric and represents a stress of Mandel type which is work conjugate to $\boldsymbol{L}_{\mathrm{v}}^{\mathrm{k}}$. Since $\overline{\boldsymbol{S}}^{\mathrm{k}}$ is symmetric, $\left[\mathcal{D}^{\text {red }}\right]^{\mathrm{k}}$ may also be written as

$$
\begin{aligned}
{\left[\mathcal{D}_{\mathrm{v}}^{r e d}\right]^{\mathrm{k}} } & =\left[\boldsymbol{C}_{\mathrm{e}}^{\mathrm{k}} \cdot \overline{\boldsymbol{S}}^{\mathrm{k}}\right]: \boldsymbol{L}_{\mathrm{v}}^{\mathrm{k}} \\
& =\overline{\boldsymbol{S}}^{\mathrm{k}}:\left[\boldsymbol{C}_{\mathrm{e}}^{\mathrm{k}} \cdot \boldsymbol{L}_{\mathrm{v}}^{\mathrm{k}}\right]^{\mathrm{sym}} \geq 0 .
\end{aligned}
$$

The viscoelastic model shall be described in the current configuration. Pushforward operation of the reduced inequality (3.20) renders

$$
\begin{aligned}
{\left[\mathcal{D}_{t}^{r e d}\right]^{\mathrm{k}} } & =\boldsymbol{F}_{\mathrm{e} \star}\left(\left[\boldsymbol{C}_{\mathrm{e}}^{\mathrm{k}} \cdot \overline{\boldsymbol{S}}^{\mathrm{k}}\right]: \boldsymbol{L}_{\mathrm{v}}^{\mathrm{k}}\right) \\
& =\left[\left[\boldsymbol{F}_{\mathrm{e}}^{\mathrm{k}}\right]^{-\mathrm{T}} \cdot\left[\left[\boldsymbol{F}_{\mathrm{e}}^{\mathrm{k}}\right]^{\mathrm{T}} \cdot \boldsymbol{g} \cdot \boldsymbol{F}_{\mathrm{e}}^{\mathrm{k}} \cdot \overline{\boldsymbol{S}}^{\mathrm{k}}\right] \cdot\left[\boldsymbol{F}_{\mathrm{e}}^{\mathrm{k}}\right]^{\mathrm{T}}\right]:\left[\boldsymbol{F}_{\mathrm{e}}^{\mathrm{k}} \cdot \boldsymbol{L}_{\mathrm{v}}^{\mathrm{k}} \cdot\left[\boldsymbol{F}_{\mathrm{e}}^{\mathrm{k}}\right]^{-1}\right],
\end{aligned}
$$


with the corresponding viscous velocity gradient

$$
\boldsymbol{l}_{\mathrm{v}}^{\mathrm{k}}=\boldsymbol{F}_{\mathrm{e}}^{\mathrm{k}} \cdot \boldsymbol{L}_{\mathrm{v}}^{\mathrm{k}} \cdot\left[\boldsymbol{F}_{\mathrm{e}}^{\mathrm{k}}\right]^{-1} .
$$

In terms of the spatial Kirchhoff stresses $\tau^{\mathrm{k}}$ and alternatively the spatial Mandel stresses $\boldsymbol{m}^{\mathrm{k}}=\boldsymbol{g} \cdot \boldsymbol{\tau}^{\mathrm{k}}$ one obtains,

$$
\begin{aligned}
{\left[\mathcal{D}_{t}^{r e d}\right]^{\mathrm{k}} } & =\left[\boldsymbol{g} \cdot \boldsymbol{F}_{\mathrm{e}}^{\mathrm{k}} \cdot \overline{\boldsymbol{S}}^{\mathrm{k}} \cdot\left[\boldsymbol{F}_{\mathrm{e}}^{\mathrm{k}}\right]^{\mathrm{T}}\right]: \boldsymbol{l}_{\mathrm{v}}^{\mathrm{k}}=2\left[\boldsymbol{g} \cdot \boldsymbol{F}_{\mathrm{e}}^{\mathrm{k}} \cdot \frac{\partial \Psi^{\mathrm{k}}}{\partial \boldsymbol{C}_{\mathrm{e}}^{\mathrm{k}}} \cdot\left[\boldsymbol{F}_{\mathrm{e}}^{\mathrm{k}}\right]^{\mathrm{T}}\right]: \boldsymbol{l}_{\mathrm{v}}^{\mathrm{k}} \\
& =2\left[\boldsymbol{g} \cdot \frac{\partial \Psi^{\mathrm{k}}}{\partial \boldsymbol{g}}\right]: \boldsymbol{l}_{\mathrm{v}}^{\mathrm{k}} \\
& =\left[\boldsymbol{g} \cdot \boldsymbol{\tau}^{\mathrm{k}}\right]: \boldsymbol{l}_{\mathrm{v}}^{\mathrm{k}} \\
& =\boldsymbol{m}^{\mathrm{k}}: \boldsymbol{l}_{\mathrm{v}}^{\mathrm{k}} .
\end{aligned}
$$

Using the elastic left Cauchy-Green tensor $\boldsymbol{b}_{\mathrm{e}}^{\mathrm{k}}=\boldsymbol{F}_{\mathrm{e}}^{\mathrm{k}} \cdot \boldsymbol{G}_{\mathrm{v}}^{-1} \cdot\left[\boldsymbol{F}_{\mathrm{e}}^{\mathrm{k}}\right]^{\mathrm{T}}$ it follows that

$$
\begin{aligned}
{\left[\mathcal{D}_{t}^{r e d}\right]^{\mathrm{k}} } & =\boldsymbol{m}^{\mathrm{k}}: \boldsymbol{l}_{\mathrm{v}}^{\mathrm{k}} \\
& =\left[\boldsymbol{m}^{\mathrm{k}} \cdot\left[\boldsymbol{b}_{\mathrm{e}}^{\mathrm{k}}\right]^{-1}\right]:\left[\boldsymbol{l}_{\mathrm{v}}^{\mathrm{k}} \cdot \boldsymbol{b}_{\mathrm{e}}^{\mathrm{k}}\right] \\
& =\left[\boldsymbol{m}^{\mathrm{k}} \cdot\left[\boldsymbol{b}_{\mathrm{e}}^{\mathrm{k}}\right]^{-1}\right]:\left[\boldsymbol{l}_{\mathrm{v}}^{\mathrm{k}} \cdot \boldsymbol{b}_{\mathrm{e}}^{\mathrm{k}}\right]^{\mathrm{sym}},
\end{aligned}
$$

since $\boldsymbol{m}^{\mathrm{k}}$ and $\boldsymbol{b}_{\mathrm{e}}^{\mathrm{k}}$ commute for isotropy.

Remark 3.2.1 Since isotropy is assumed it follows that

$$
\boldsymbol{m}^{\mathrm{k}}=\boldsymbol{g} \cdot \frac{\partial \Psi^{\mathrm{k}}}{\partial \boldsymbol{g}}=\frac{\partial \Psi^{\mathrm{k}}}{\partial \boldsymbol{b}_{\mathrm{e}}^{\mathrm{k}}} \cdot \boldsymbol{b}_{\mathrm{e}}^{\mathrm{k}} .
$$

(see equation (2.25)).

Now it shall be shown that the following relation holds

$$
\left[\boldsymbol{l}_{\mathrm{v}}^{\mathrm{k}} \cdot \boldsymbol{b}_{\mathrm{e}}^{\mathrm{k}}\right]^{\mathrm{sym}}=-\frac{1}{2} \mathrm{~L}_{t} \boldsymbol{b}_{\mathrm{e}}^{\mathrm{k}},
$$

whereby $\mathrm{L}_{t} \boldsymbol{b}_{\mathrm{e}}^{\mathrm{k}}$ is the Lie-derivative of the elastic left Cauchy-Green tensor along the velocity field of the material motion.

Proof 3.2.1 Pullback operation of $\boldsymbol{b}_{\mathrm{e}}^{\mathrm{k}}$ to the reference configuration is computed which yields $\left[\boldsymbol{C}_{\mathrm{v}}^{\mathrm{k}}\right]^{-1}$, whereon the material time derivative is taken and the result pushed forward to the spatial configuration. This is the definition of the Lie-derivative of the tensor $\boldsymbol{b}_{\mathrm{e}}^{\mathrm{k}}$ (see subsection 3.1.1)

$$
\begin{aligned}
\mathrm{L}_{t} \boldsymbol{b}_{\mathrm{e}}^{\mathrm{k}} & =\boldsymbol{F} \cdot \mathrm{D}_{t}\left(\boldsymbol{F}^{-1} \cdot \boldsymbol{F}_{\mathrm{e}}^{\mathrm{k}} \cdot \boldsymbol{G}_{\mathrm{v}}^{-1} \cdot\left[\boldsymbol{F}_{\mathrm{e}}^{\mathrm{k}}\right]^{\mathrm{T}} \cdot \boldsymbol{F}^{-\mathrm{T}}\right) \cdot \boldsymbol{F}^{\mathrm{T}} \\
& =\boldsymbol{F} \cdot \mathrm{D}_{t}\left(\left[\boldsymbol{F}_{\mathrm{v}}^{\mathrm{k}}\right]^{-1} \cdot \boldsymbol{G}_{\mathrm{v}}^{-1} \cdot\left[\boldsymbol{F}_{\mathrm{v}}^{\mathrm{k}}\right]^{-\mathrm{T}}\right) \cdot \boldsymbol{F}^{\mathrm{T}} .
\end{aligned}
$$

Taking into account equation (3.21), the computation of the material time derivatives $\left[\boldsymbol{F}_{\mathrm{v}}^{\mathrm{k}}\right]^{-1}$ and $\left[\boldsymbol{F}_{\mathrm{v}}^{\mathrm{k}}\right]^{-\mathrm{T}}$ is done using

$$
\begin{aligned}
& \mathrm{D}_{t}\left(\boldsymbol{F}_{\mathrm{v}}^{\mathrm{k}} \cdot\left[\boldsymbol{F}_{\mathrm{v}}^{\mathrm{k}}\right]^{-1}\right)=\mathbf{0} \\
\Rightarrow & \mathrm{D}_{t} \boldsymbol{F}_{\mathrm{v}}^{\mathrm{k}} \cdot\left[\boldsymbol{F}_{\mathrm{v}}^{\mathrm{k}}\right]^{-1}=-\boldsymbol{F}_{\mathrm{v}}^{\mathrm{k}} \cdot \mathrm{D}_{t}\left[\boldsymbol{F}_{\mathrm{v}}^{\mathrm{k}}\right]^{-1}=\boldsymbol{L}_{\mathrm{v}}^{\mathrm{k}} \\
\Rightarrow & \mathrm{D}_{t}\left[\boldsymbol{F}_{\mathrm{v}}^{\mathrm{k}}\right]^{-1}=-\left[\boldsymbol{F}_{\mathrm{v}}^{\mathrm{k}}\right]^{-1} \cdot \boldsymbol{L}_{\mathrm{v}}^{\mathrm{k}} \cdot
\end{aligned}
$$


With definition (3.24) it follows that

$$
\mathrm{D}_{t}\left[\boldsymbol{F}_{\mathrm{v}}^{\mathrm{k}}\right]^{-1}=-\left[\boldsymbol{F}_{\mathrm{v}}^{\mathrm{k}}\right]^{-1} \cdot\left[\boldsymbol{F}_{\mathrm{e}}^{\mathrm{k}}\right]^{-1} \cdot \boldsymbol{l}_{\mathrm{v}}^{\mathrm{k}} \cdot \boldsymbol{F}_{\mathrm{e}}^{\mathrm{k}}
$$

The substitution of equation (3.31) into equation (3.29) leads to

$$
\begin{aligned}
\mathrm{L}_{t} \boldsymbol{b}_{\mathrm{e}}^{\mathrm{k}}= & -\boldsymbol{F} \cdot\left[\boldsymbol{F}_{\mathrm{v}}^{\mathrm{k}}\right]^{-1} \cdot\left[\boldsymbol{F}_{\mathrm{e}}^{\mathrm{k}}\right]^{-1} \cdot \boldsymbol{l}_{\mathrm{v}}^{\mathrm{k}} \cdot \boldsymbol{F}_{\mathrm{e}}^{\mathrm{k}} \cdot \boldsymbol{G}_{\mathrm{v}}^{-1} \cdot\left[\boldsymbol{F}_{\mathrm{v}}^{\mathrm{k}}\right]^{-\mathrm{T}} \cdot \boldsymbol{F}^{\mathrm{T}} \\
& -\boldsymbol{F} \cdot\left[\boldsymbol{F}_{\mathrm{v}}^{\mathrm{k}}\right]^{-1} \cdot \boldsymbol{G}_{\mathrm{v}}^{-1} \cdot\left[\boldsymbol{F}_{\mathrm{e}}^{\mathrm{k}}\right]^{\mathrm{T}} \cdot\left[\boldsymbol{l}_{\mathrm{v}}^{\mathrm{k}}\right]^{\mathrm{T}} \cdot\left[\boldsymbol{F}_{\mathrm{e}}^{\mathrm{k}}\right]^{-\mathrm{T}} \cdot\left[\boldsymbol{F}_{\mathrm{v}}^{\mathrm{k}}\right]^{-\mathrm{T}} \cdot \boldsymbol{F}^{\mathrm{T}} \\
= & -\boldsymbol{l}_{\mathrm{v}}^{\mathrm{k}} \cdot \boldsymbol{b}_{\mathrm{e}}^{\mathrm{k}}-\boldsymbol{b}_{\mathrm{e}}^{\mathrm{k}} \cdot\left[\boldsymbol{l}_{\mathrm{v}}^{\mathrm{k}}\right]^{\mathrm{T}} \\
= & -2\left[\boldsymbol{l}_{\mathrm{v}}^{\mathrm{k}} \cdot \boldsymbol{b}_{\mathrm{e}}^{\mathrm{k}}\right]^{\mathrm{sym}}
\end{aligned}
$$

q.e.d..

Using equation (3.28) the expression (3.29) for the reduced dissipation takes the form

$$
\begin{aligned}
{\left[\mathcal{D}_{t}^{r e d}\right]^{\mathrm{k}} } & =\left[\boldsymbol{m}^{\mathrm{k}} \cdot\left[\boldsymbol{b}_{\mathrm{e}}^{\mathrm{k}}\right]^{-1}\right]:\left[\boldsymbol{l}_{\mathrm{v}}^{\mathrm{k}} \cdot \boldsymbol{b}_{\mathrm{e}}^{\mathrm{k}}\right]^{\mathrm{sym}} \\
& =-\frac{1}{2}\left[\boldsymbol{m}^{\mathrm{k}} \cdot\left[\boldsymbol{b}_{\mathrm{e}}^{\mathrm{k}}\right]^{-1}\right]: \mathrm{L}_{t} \boldsymbol{b}_{\mathrm{e}}^{\mathrm{k}} \\
& =-\frac{1}{2} \boldsymbol{m}^{\mathrm{k}}:\left[\mathrm{L}_{t} \boldsymbol{b}_{\mathrm{e}}^{\mathrm{k}} \cdot\left[\boldsymbol{b}_{\mathrm{e}}^{\mathrm{k}}\right]^{-1}\right] \\
& =-\left[\boldsymbol{g} \cdot \boldsymbol{\tau}^{\mathrm{k}}\right]: \frac{1}{2}\left[\mathrm{~L}_{t} \boldsymbol{b}_{\mathrm{e}}^{\mathrm{k}} \cdot\left[\boldsymbol{b}_{\mathrm{e}}^{\mathrm{k}}\right]^{-1}\right] \geq 0
\end{aligned}
$$

\subsubsection{Evolution equations for multiplicative viscoelasticity}

In order to specify the proportionality between the viscous strain rates and the corresponding stresses, evolution equations are required (see Reese and Govindjee [65]). Thereby the criterion of thermodynamic admissibility has to be fulfilled which means that inequality (3.17) must be fulfilled.

\section{Intermediate configuration}

The desired evolution equation must guarantee that the dissipation inequality for the intermediate configuration (see equation (3.20))

$$
\left[\mathcal{D}^{r e d}\right]^{\mathrm{k}}=\boldsymbol{M}^{\mathrm{k}}: \boldsymbol{L}_{\mathrm{v}}^{\mathrm{k}} \geq 0
$$

is satisfied for all possible $\boldsymbol{L}_{\mathrm{v}}^{\mathrm{k}}$. Such a choice is afforded if the evolution equation for $\boldsymbol{L}_{\mathrm{v}}^{\mathrm{k}}$ renders equation (3.34) a positive quadratic form which is done as follows

$$
\boldsymbol{L}_{\mathrm{v}}^{\mathrm{k}} \doteq\left[\mathbf{W}^{\mathrm{k}}\right]^{-1}: \boldsymbol{M}^{\mathrm{k}}
$$

whereby $\mathbf{W}^{\mathrm{k}}$ is a 4 th order viscosity tensor that is chosen to be symmetric and positive definite. 


\section{Spatial configuration}

Since isotropy is assumed a spatial description for the states of stresses and strains is preferred. The dissipation inequality in $\mathcal{B}_{t}$ (see equation (3.33)) takes a positive quadratic form by choosing the following evolution equation

$$
-\frac{1}{2} \mathrm{~L}_{t} \boldsymbol{b}_{\mathrm{e}}^{\mathrm{k}} \cdot\left[\boldsymbol{b}_{\mathrm{e}}^{\mathrm{k}}\right]^{-1} \doteq\left[\mathbf{w}^{\mathrm{k}}\right]^{-1}:\left[\boldsymbol{g} \cdot \boldsymbol{\tau}^{\mathrm{k}}\right] .
$$

Equivalent to equation (3.35) the 4 th order viscosity tensor $\mathbf{w}^{\mathrm{k}}$ is chosen to be symmetric and positive definite with

$$
\left[\mathbf{w}^{\mathrm{k}}\right]^{-1}=\frac{1}{2 \eta_{\mathrm{D}}^{\mathrm{k}}} \overbrace{\left[\mathbf{i}_{i}-\frac{1}{3} \boldsymbol{i} \otimes \boldsymbol{i}\right]}^{\mathbf{i}_{\boldsymbol{i}}^{\mathrm{dev}}}+\frac{1}{3 \eta_{\mathrm{v}}^{\mathrm{k}}} \overbrace{\frac{1}{3} \boldsymbol{i} \otimes \boldsymbol{i}}^{\mathbf{i}_{\boldsymbol{i}}^{\mathrm{vol}}},
$$

$\eta_{\mathrm{D}}^{\mathrm{k}}$ and $\eta_{\mathrm{v}}^{\mathrm{k}}$ are viscosity parameters which are chosen to be constant in this work. The 4th order symmetric identity tensor $\mathbf{i}_{\boldsymbol{i}}=\mathbf{i}_{\boldsymbol{i}}^{\text {dev }}+\mathbf{i}_{\boldsymbol{i}}^{\text {vol }}$ is computed by

$$
2 \mathbf{i}_{i}=\boldsymbol{i} \bar{\otimes} \boldsymbol{i}+\boldsymbol{i} \underline{\otimes} \boldsymbol{i},
$$

whereby $\boldsymbol{i}$ is the 2 nd order identity tensor.

\subsection{Algorithmic formulation}

With regard to the implementation in a finite element code the algorithmic equations are described in this section. The implicit time integration on the basis of an operator split method and the later choice of Ogden type free energy functions for isotropy lead to a description of the stresses and strain measures in principal directions. In consequence of equation (3.12) the Kirchhoff stresses allow representation as

$$
\boldsymbol{\tau}=\boldsymbol{F} \cdot \boldsymbol{S} \cdot \boldsymbol{F}^{\mathrm{T}}=\boldsymbol{\tau}^{\mathrm{eq}}+\sum_{\mathrm{k}=1}^{\mathrm{N}} \boldsymbol{\tau}^{\mathrm{k}}
$$

Since isotropy is assumed, - as shown in chapter 2.2 and mentioned in equation (3.27) the non-equilibrium terms can be expressed as

$$
\boldsymbol{\tau}^{\mathrm{k}}=2 \boldsymbol{F}_{\mathrm{e}}^{\mathrm{k}} \cdot \frac{\partial \Psi^{\mathrm{k}}}{\partial \boldsymbol{C}_{\mathrm{e}}^{\mathrm{k}}} \cdot\left[\boldsymbol{F}_{\mathrm{e}}^{\mathrm{k}}\right]^{\mathrm{T}}=2 \boldsymbol{g}^{-1} \cdot \frac{\partial \Psi^{\mathrm{k}}}{\partial \boldsymbol{b}_{\mathrm{e}}^{\mathrm{k}}} \cdot \boldsymbol{b}_{\mathrm{e}}^{\mathrm{k}} .
$$

As mentioned in section 2.3, for isotropy the free energy function can be formulated as a function of the principle stretches of the left Cauchy-Green tensor

$$
\boldsymbol{b}=\sum_{\mathrm{a}=1}^{3} \mathrm{~b}_{\mathrm{a}} \boldsymbol{n}_{\mathrm{a}} \otimes \boldsymbol{n}_{\mathrm{a}}=\sum_{\mathrm{a}=1}^{3} \lambda_{\mathrm{a}}^{2} \boldsymbol{n}_{\mathrm{a}} \otimes \boldsymbol{n}_{\mathrm{a}}
$$

and of the elastic left Cauchy-Green tensor

$$
\boldsymbol{b}_{\mathrm{e}}^{\mathrm{k}}=\sum_{\mathrm{a}=1}^{3} \mathrm{~b}_{\mathrm{a}}^{\mathrm{k}} \boldsymbol{n}_{\mathrm{a}} \otimes \boldsymbol{n}_{\mathrm{a}}=\sum_{\mathrm{a}=1}^{3}\left[\lambda_{\mathrm{a}}^{\mathrm{k}}\right]^{2} \boldsymbol{n}_{\mathrm{a}} \otimes \boldsymbol{n}_{\mathrm{a}}
$$


which leads to

$$
\boldsymbol{\tau}^{\mathrm{eq}}=\sum_{\mathrm{a}=1}^{3} \tau_{\mathrm{a}}^{\mathrm{eq}} \boldsymbol{n}_{\mathrm{a}} \otimes \boldsymbol{n}_{\mathrm{a}}=\sum_{\mathrm{a}=1}^{3} \lambda_{\mathrm{a}} \frac{\partial \Psi^{\mathrm{eq}}}{\partial \lambda_{\mathrm{a}}} \boldsymbol{n}_{\mathrm{a}} \otimes \boldsymbol{n}_{\mathrm{a}}
$$

and

$$
\boldsymbol{\tau}^{\mathrm{k}}=\sum_{\mathrm{a}=1}^{3} \tau_{\mathrm{a}}^{\mathrm{k}} \boldsymbol{n}_{\mathrm{a}} \otimes \boldsymbol{n}_{\mathrm{a}}=\sum_{\mathrm{a}=1}^{3} \lambda_{\mathrm{a}_{\mathrm{e}}}^{\mathrm{k}} \frac{\partial \Psi^{\mathrm{k}}}{\partial \lambda_{\mathrm{a}_{\mathrm{e}}}^{\mathrm{k}}} \boldsymbol{n}_{\mathrm{a}} \otimes \boldsymbol{n}_{\mathrm{a}} .
$$

Note that due to isotropy $\boldsymbol{\tau}^{\mathrm{eq}}$ is coaxial to $\boldsymbol{\tau}^{\mathrm{k}}, \boldsymbol{b}$ and $\boldsymbol{b}_{\mathrm{e}}^{\mathrm{k}}$. The determinant of $\boldsymbol{F}_{\mathrm{e}}^{\mathrm{k}}$ is referred to as

$$
J_{\mathrm{e}}^{\mathrm{k}}=\lambda_{1_{\mathrm{e}}}^{\mathrm{k}} \lambda_{2_{\mathrm{e}}}^{\mathrm{k}} \lambda_{3_{\mathrm{e}}}^{\mathrm{k}} .
$$

Consequently, the eigenvalues of the isochoric elastic left Cauchy-Green tensor are given by

$$
\overline{\mathrm{b}}_{\mathrm{a}_{\mathrm{e}}}^{\mathrm{k}}=\left[J_{\mathrm{e}}^{\mathrm{k}}\right]^{-2 / 3} \mathrm{~b}_{\mathrm{a}_{\mathrm{e}}}=\left[J_{\mathrm{e}}^{\mathrm{k}}\right]^{-2 / 3}\left[\lambda_{\mathrm{a}_{\mathrm{e}}}^{\mathrm{k}}\right]^{2}
$$

with

$$
\overline{\mathbf{b}}_{\mathrm{e}}^{\mathrm{k}}=\left[J_{\mathrm{e}}^{\mathrm{k}}\right]^{-2 / 3} \mathbf{b}_{\mathrm{e}}^{\mathrm{k}},
$$

so that the deviatoric and volumetric part of the principal Kirchhoff stresses take the form

$$
\tau_{\mathrm{a}}^{\mathrm{k}}=\underbrace{\sum_{\mathrm{b}=1}^{3} \frac{\partial\left[\Psi^{\mathrm{k}}\right]^{\mathrm{dev}}}{\partial \overline{\mathrm{b}}_{\mathrm{b}_{\mathrm{e}}^{\mathrm{k}}}^{\mathrm{k}}} \frac{\partial \overline{\mathrm{b}}_{\mathrm{b}_{\mathrm{e}}^{\mathrm{k}}}^{\mathrm{k}}}{\partial \lambda_{\mathrm{a}_{\mathrm{e}}}^{\mathrm{k}}} \lambda_{\mathrm{a}_{\mathrm{e}}}^{\mathrm{k}}}_{\left[\tau_{\mathrm{a}}^{\mathrm{k}}\right]^{\mathrm{dev}}}+\underbrace{\frac{\partial\left[\Psi^{\mathrm{k}}\right]^{\mathrm{vol}}}{\partial J_{\mathrm{e}}^{\mathrm{k}}} J_{\mathrm{e}}^{\mathrm{k}}}_{\frac{1}{3} \boldsymbol{\tau}^{\mathrm{k}}: \boldsymbol{g}} .
$$

\subsubsection{Exponential time integration}

In this subsection the local computation of the non-equilibrium stresses by integration of the evolution equation is shown. Thereby, the finite time interval with $t \in\left[t_{\mathrm{n}-1}, t_{\mathrm{n}}\right]$ and $\Delta t=t_{\mathrm{n}}-t_{\mathrm{n}-1} \geq 0$ is considered and it is supposed that the actual deformation gradient $\boldsymbol{F}$ at time $t=t_{\mathrm{n}}$ is given.

\section{Predictor-corrector-split of $b_{\mathrm{e}}^{\mathrm{k}}$}

Taking into account that $\boldsymbol{\varphi}^{*} \boldsymbol{b}_{\mathrm{e}}^{\mathrm{k}}=\boldsymbol{F}^{-1} \cdot \boldsymbol{b}_{\mathrm{e}}^{\mathrm{k}} \cdot \boldsymbol{F}^{-\mathrm{T}}=\left[\boldsymbol{F}_{\mathrm{v}}^{\mathrm{k}}\right]^{-1} \cdot\left[\boldsymbol{G}_{\mathrm{v}}^{\mathrm{k}}\right]^{-1} \cdot\left[\boldsymbol{F}_{\mathrm{v}}^{\mathrm{k}}\right]^{-\mathrm{T}}=\left[\boldsymbol{C}_{\mathrm{v}}^{\mathrm{k}}\right]^{-1}$ holds, the material time derivative of the internal variable ${ }^{1}$ is considered

$$
\begin{aligned}
\mathrm{D}_{t} \boldsymbol{b}_{\mathrm{e}}^{\mathrm{k}} & =\mathrm{L}_{t} \boldsymbol{b}_{\mathrm{e}}^{\mathrm{k}}+\boldsymbol{l} \cdot \boldsymbol{b}_{\mathrm{e}}^{\mathrm{k}}+\boldsymbol{b}_{\mathrm{e}}^{\mathrm{k}} \cdot \boldsymbol{l}^{\mathrm{T}} \\
& =\boldsymbol{F} \cdot \mathrm{D}_{t}\left(\left[\boldsymbol{C}_{\mathrm{v}}^{\mathrm{k}}\right]^{-1}\right) \cdot \boldsymbol{F}^{\mathrm{T}}+\mathrm{D}_{t} \boldsymbol{F} \cdot\left[\boldsymbol{C}_{\mathrm{v}}^{\mathrm{k}}\right]^{-1} \cdot \boldsymbol{F}^{\mathrm{T}}+\boldsymbol{F} \cdot\left[\boldsymbol{C}_{\mathrm{v}}^{\mathrm{k}}\right]^{-1} \cdot \mathrm{D}_{t} \boldsymbol{F}^{\mathrm{T}},
\end{aligned}
$$

Remark 3.3.1 : With $\mathrm{D}_{t} \boldsymbol{F}^{-1}=-\boldsymbol{F}^{-1} \cdot \boldsymbol{l}$ it follows that

$$
\begin{aligned}
\mathrm{L}_{t} \boldsymbol{b}_{\mathrm{e}}^{\mathrm{k}} & =\boldsymbol{F} \cdot \mathrm{D}_{t}\left(\boldsymbol{F}^{-1} \cdot \boldsymbol{b}_{\mathrm{e}}^{\mathrm{k}} \cdot \boldsymbol{F}^{-\mathrm{T}}\right) \cdot \boldsymbol{F}^{\mathrm{T}} \\
& =\boldsymbol{F} \cdot \mathrm{D}_{t}\left(\boldsymbol{F}^{-1}\right) \cdot \boldsymbol{b}_{\mathrm{e}}^{\mathrm{k}}+\mathrm{D}_{t} \boldsymbol{b}_{\mathrm{e}}^{\mathrm{k}}+\boldsymbol{b}_{\mathrm{e}}^{\mathrm{k}} \cdot \mathrm{D}_{t}\left(\boldsymbol{F}^{-\mathrm{T}}\right) \cdot \boldsymbol{F}^{\mathrm{T}} \\
& =\mathrm{D}_{t} \boldsymbol{b}_{\mathrm{e}}^{\mathrm{k}}-\boldsymbol{l} \cdot \boldsymbol{b}_{\mathrm{e}}^{\mathrm{k}}-\boldsymbol{b}_{\mathrm{e}}^{\mathrm{k}} \cdot \boldsymbol{l}^{\mathrm{T}},
\end{aligned}
$$


which motivates the use of a predictor-corrector integration method (see also Weber and Anand [73]). The elastic predictor

$$
\mathrm{D}_{t} \boldsymbol{b}_{\mathrm{e}}^{\mathrm{k}}=\mathrm{D}_{t} \boldsymbol{F} \cdot\left[\boldsymbol{C}_{\mathrm{v}}^{\mathrm{k}}\right]^{-1} \cdot \boldsymbol{F}^{\mathrm{T}}+\boldsymbol{F} \cdot\left[\boldsymbol{C}_{\mathrm{v}}^{\mathrm{k}}\right]^{-1} \cdot \mathrm{D}_{t} \boldsymbol{F}^{\mathrm{T}}
$$

has as solution the trial values,

$$
\left[\boldsymbol{b}_{\mathrm{e}}^{\mathrm{k}}\right]_{\mathrm{tr}}=[\boldsymbol{F}]_{t=t_{\mathrm{n}}} \cdot\left[\left[\boldsymbol{C}_{\mathrm{v}}^{\mathrm{k}}\right]^{-1}\right]_{t=t_{\mathrm{n}-1}} \cdot\left[\boldsymbol{F}^{\mathrm{T}}\right]_{t=t_{\mathrm{n}}}
$$

where $\mathrm{n}$ denotes the actual time step. The elastic predictor physically means an elastic step at time $t_{\mathrm{n}}$ in which the viscous flow is frozen. Thus the inverse viscous right CauchyGreen tensor $\left[\boldsymbol{C}_{\mathrm{v}}^{\mathrm{k}}\right]^{-1}$ is a field in which the load history - as the irreversible part of the viscoelastic deformation - is stored. Now a viscous corrector step follows in which $\mathrm{D}_{t} \boldsymbol{F}=0$ holds with

$$
\mathrm{D}_{t} \boldsymbol{b}_{\mathrm{e}}^{\mathrm{k}}=\boldsymbol{F} \cdot \mathrm{D}_{t}\left(\left[\boldsymbol{C}_{\mathrm{v}}^{\mathrm{k}}\right]^{-1}\right) \cdot \boldsymbol{F}^{\mathrm{T}}=\mathrm{L}_{t} \boldsymbol{b}_{\mathrm{e}}^{\mathrm{k}},
$$

and together with the viscous flow function (see equation (3.36)) one obtains

$$
\mathrm{D}_{t} \boldsymbol{b}_{\mathrm{e}}^{\mathrm{k}}=-\left[2\left[\mathbf{w}^{\mathrm{k}}\right]^{-1}:\left[\boldsymbol{g} \cdot \boldsymbol{\tau}^{\mathrm{k}}\right]\right] \cdot \boldsymbol{b}_{\mathrm{e}}^{\mathrm{k}},
$$

which can be solved by means of an implicit Euler backward method with exponential shift to result in

$$
\left[\boldsymbol{b}_{\mathrm{e}}^{\mathrm{k}}\right]_{t=t_{\mathrm{n}}} \approx \exp \left(-2\left[t_{\mathrm{n}}-t_{\mathrm{n}-1}\right]\left[\left[\mathbf{w}^{\mathrm{k}}\right]^{-1}:\left[\boldsymbol{g} \cdot \boldsymbol{\tau}^{\mathrm{k}}\right]\right]_{t=t_{\mathrm{n}}}\right) \cdot\left[\boldsymbol{b}_{\mathrm{e}}^{\mathrm{k}}\right]_{\mathrm{tr}}
$$

Since for isotropy the principal axes are the same for $\boldsymbol{\tau}^{\mathrm{k}}, \boldsymbol{b}_{\mathrm{e}}^{\mathrm{k}}$, and $\left[\boldsymbol{b}_{\mathrm{e}}^{\mathrm{k}}\right]_{\operatorname{tr}}$ with

$$
\left[\boldsymbol{b}_{\mathrm{e}}^{\mathrm{k}}\right]_{\mathrm{tr}}=\sum_{\mathrm{a}=1}^{3}\left[\mathrm{~b}_{\mathrm{a}_{\mathrm{e}}^{\mathrm{e}}}^{\mathrm{k}}\right]_{\mathrm{tr}} \boldsymbol{n}_{\mathrm{a}} \otimes \boldsymbol{n}_{\mathrm{a}}=\sum_{\mathrm{a}=1}^{3}\left[\lambda_{\mathrm{a}_{\mathrm{e}}}^{\mathrm{k}}\right]_{\mathrm{tr}}^{2} \boldsymbol{n}_{\mathrm{a}} \otimes \boldsymbol{n}_{\mathrm{a}},
$$

this leads to the relation

$$
\left[\lambda_{\mathrm{a}_{\mathrm{e}}}^{\mathrm{k}}\right]^{2}=\exp \left(-\Delta t\left[\frac{1}{\eta_{\mathrm{D}}^{\mathrm{k}}}\left[\tau_{\mathrm{a}}^{\mathrm{k}}\right]^{\mathrm{dev}}+\frac{2}{9 \eta_{\mathrm{v}}^{\mathrm{k}}} \boldsymbol{\tau}^{\mathrm{k}}: \boldsymbol{g}\right]\right)\left[\lambda_{\mathrm{a}_{\mathrm{e}}}^{\mathrm{k}}\right]_{\mathrm{tr}}^{2} .
$$

In terms of logarithmic stretches $\varepsilon_{\mathrm{a}_{\mathrm{e}}}^{\mathrm{k}}=\ln \lambda_{\mathrm{a}_{\mathrm{e}}}^{\mathrm{k}}$ the evolution equations read

$$
\varepsilon_{\mathrm{a}_{\mathrm{e}}}^{\mathrm{k}}=-\Delta t\left[\frac{1}{2 \eta_{\mathrm{D}}^{\mathrm{k}}}\left[\tau_{\mathrm{a}}^{\mathrm{k}}\right]^{\mathrm{dev}}+\frac{1}{9 \eta_{\mathrm{v}}^{\mathrm{k}}} \boldsymbol{\tau}^{\mathrm{k}}: \boldsymbol{g}\right]+\left[\varepsilon_{\mathrm{a}_{\mathrm{e}}}^{\mathrm{k}}\right]_{\mathrm{tr}} .
$$

These nonlinear equations are solved by a local Newton scheme, see algorithm 3.3.1 for further details. 
Algorithm 3.3.1 Local Newton-iteration.

(1) Nonlin. eq. $\mathrm{r}_{\mathrm{a}}^{\mathrm{k}}=\varepsilon_{\mathrm{a}_{\mathrm{e}}}^{\mathrm{k}}+\Delta t\left[\frac{1}{2 \eta_{\mathrm{D}}^{\mathrm{k}}}\left[\tau_{\mathrm{a}}^{\mathrm{k}}\right]^{\mathrm{dev}}+\frac{1}{9 \eta_{\mathrm{v}}^{\mathrm{k}}} \boldsymbol{\tau}^{\mathrm{k}}: \boldsymbol{g}\right]-\left[\varepsilon_{\mathrm{a}_{\mathrm{e}}}^{\mathrm{k}}\right]_{\mathrm{tr}}=0$

(2) Linearization $\mathrm{r}_{\mathrm{a}}^{\mathrm{k}} \approx \underbrace{\left.\mathrm{r}_{\mathrm{a}}^{\mathrm{k}}\right|_{\left[\varepsilon_{\mathrm{a}}^{\mathrm{k}}\right]_{l}}}_{\left[\mathrm{r}_{\mathrm{a}}^{\mathrm{k}}\right]_{l}}+\sum_{\mathrm{b}=1}^{3} \underbrace{\left.\frac{\partial \mathrm{r}_{\mathrm{a}}^{\mathrm{k}}}{\partial \varepsilon_{\mathrm{b}_{\mathrm{e}}}^{\mathrm{k}}}\right|_{\left[\varepsilon_{\mathrm{a}_{\mathrm{e}}}^{\mathrm{k}}\right]_{l}}}_{\left[K_{\mathrm{ab}}^{\mathrm{k}}\right]_{l}}\left[\Delta \varepsilon_{\mathrm{b}_{\mathrm{e}}}^{\mathrm{k}}\right]_{l}=0$

(3) Solution

$$
\sum_{\mathrm{b}=1}^{3}\left[K_{\mathrm{ab}}^{\mathrm{k}}\right]_{l}\left[\Delta \varepsilon_{\mathrm{b}_{\mathrm{e}}}^{\mathrm{k}}\right]_{l}=-\left[\overline{\mathrm{r}}_{\mathrm{a}}^{\mathrm{k}}\right]_{l}
$$

(4) Update

$$
\left[\varepsilon_{\mathrm{ae}}^{\mathrm{k}}\right]_{l+1}=\left[\varepsilon_{\mathrm{ae}_{\mathrm{e}}}^{\mathrm{k}}\right]_{l}+\left[\Delta \varepsilon_{\mathrm{ae}}^{\mathrm{k}}\right]_{l}, l=l+1
$$

repeat (1-4) until $\left|\mathrm{r}_{\mathrm{a}}^{\mathrm{k}}\right|<$ tol

Since $\eta_{\mathrm{D}}$ and $\eta_{\mathrm{v}}$ are constants, the matrix $K_{\mathrm{ab}}^{\mathrm{k}}$ in the local Newton-iteration is calculated by

$$
K_{\mathrm{ab}}^{\mathrm{k}}=\frac{\partial \mathrm{r}_{\mathrm{a}}^{\mathrm{k}}}{\partial \varepsilon_{\mathrm{b}_{\mathrm{e}}}^{\mathrm{k}}}=\delta_{\mathrm{ab}}+\Delta t \frac{1}{2 \eta_{\mathrm{D}}^{\mathrm{k}}} \frac{\partial\left[\tau_{\mathrm{a}}^{\mathrm{k}}\right]^{\mathrm{dev}}}{\partial \varepsilon_{\mathrm{b}_{\mathrm{e}}}^{\mathrm{k}}}+\Delta t \frac{1}{9 \eta_{\mathrm{v}}^{\mathrm{k}}} \frac{\partial \boldsymbol{\tau}^{\mathrm{k}}: \boldsymbol{g}}{\partial \varepsilon_{\mathrm{b}_{\mathrm{e}}}^{\mathrm{k}}}
$$

\subsubsection{Free energy functions}

The equilibrium term and the non-equilibrium terms are chosen as Ogden free energy functions described in subsection 2.3.2. For the equilibrium term it is defined as

$$
\begin{aligned}
\Psi^{\mathrm{eq}}= & \underbrace{\sum_{\mathrm{r}=1}^{N_{\mathrm{T}}} \frac{\left[\mu_{\mathrm{eq}}\right]_{\mathrm{r}}}{\left[\alpha_{\mathrm{eq}}\right]_{\mathrm{r}}}\left[\overline{\mathrm{b}}_{1}^{\left[\alpha_{\mathrm{eq}}\right]_{\mathrm{r}} / 2}+\overline{\mathrm{b}}_{2}^{\left[\alpha_{\mathrm{eq}}\right]_{\mathrm{r}} / 2}+\overline{\mathrm{b}}_{3}^{\left[\alpha_{\mathrm{eq}}\right]_{\mathrm{r}} / 2}-3\right]}_{\left[\Psi^{\mathrm{eq}}\right]^{\mathrm{dev}}} \\
& +\underbrace{\frac{\kappa_{\mathrm{eq}}}{4}\left[J^{2}-2 \ln J-1\right]}_{\left[\Psi^{\mathrm{eq}}\right]^{\mathrm{vol}}},
\end{aligned}
$$

with $J=\lambda_{1} \lambda_{2} \lambda_{3}$ and $\overline{\mathrm{b}}_{\mathrm{a}}=J^{-2 / 3} \mathrm{~b}_{\mathrm{a}}$. Therefore, for $\mathrm{a} \neq \mathrm{b}, \mathrm{a} \neq \mathrm{c}, \mathrm{b} \neq \mathrm{c}$ the principal Kirchhoff stresses are computed by (see subsection 2.3.2)

$$
\tau_{\mathrm{a}}^{\mathrm{eq}}=\underbrace{\sum_{\mathrm{r}=1}^{\mathrm{N}_{\mathrm{T}}}\left[\mu_{\mathrm{eq}}\right]_{\mathrm{r}}\left[\frac{2}{3} \overline{\mathrm{b}}_{\mathrm{a}}^{\left[\alpha_{\mathrm{eq}}\right]_{\mathrm{r}} / 2}-\frac{1}{3} \overline{\mathrm{b}}_{\mathrm{b}}^{\left[\alpha_{\mathrm{eq}}\right]_{\mathrm{r}} / 2}-\frac{1}{3} \overline{\mathrm{b}}_{\mathrm{c}}^{\left[\alpha_{\mathrm{eq}}\right]_{\mathrm{r}} / 2}\right]}_{\left[\tau_{\mathrm{a}}^{\mathrm{eq}}\right]}+\underbrace{\frac{\kappa_{\mathrm{eq}}}{2}\left[J^{2}-1\right]}_{\frac{1}{3} \boldsymbol{\tau}^{\mathrm{eq}}: \boldsymbol{g}} .
$$


For the non-equilibrium terms the free energy function is equivalently chosen as

$$
\begin{aligned}
\Psi^{\mathrm{k}}= & \underbrace{\sum_{\mathrm{r}=1}^{N_{\mathrm{T}}^{\mathrm{k}}} \frac{\left[\mu_{\mathrm{neq}}^{\mathrm{k}}\right]_{\mathrm{r}}}{\left[\alpha_{\mathrm{neq}}^{\mathrm{k}}\right]_{\mathrm{r}}}\left[\left[\overline{\mathrm{b}}_{1_{\mathrm{e}}^{\mathrm{k}}}^{\mathrm{k}}\right]^{\left[\alpha_{\text {neq }}^{\mathrm{k}}\right]_{\mathrm{r}} / 2}+\left[\overline{\mathrm{b}}_{2_{\mathrm{e}}}^{\mathrm{k}}\right]_{\text {neq }}^{\left[\alpha_{\mathrm{r}}^{\mathrm{k}} / 2\right.}+\left[\overline{\mathrm{b}}_{3_{\mathrm{e}}}^{\mathrm{k}}\right]^{\left[\alpha_{\mathrm{neq}}^{\mathrm{k}}\right]_{\mathrm{r}} / 2}-3\right]}_{\left[\Psi^{\mathrm{k}}\right]^{\mathrm{dev}}} \\
& +\underbrace{\frac{\kappa_{\text {neq }}^{\mathrm{k}}\left[\left[J_{\mathrm{e}}^{\mathrm{k}}\right]^{2}-2 \ln J_{\mathrm{e}}^{\mathrm{k}}-1\right]}{4}}_{\left[\Psi^{\mathrm{k}}\right]^{\mathrm{vol}}}
\end{aligned}
$$

which leads to a calculation of the principal Kirchhoff stresses for $a \neq b, a \neq c, b \neq c$ by

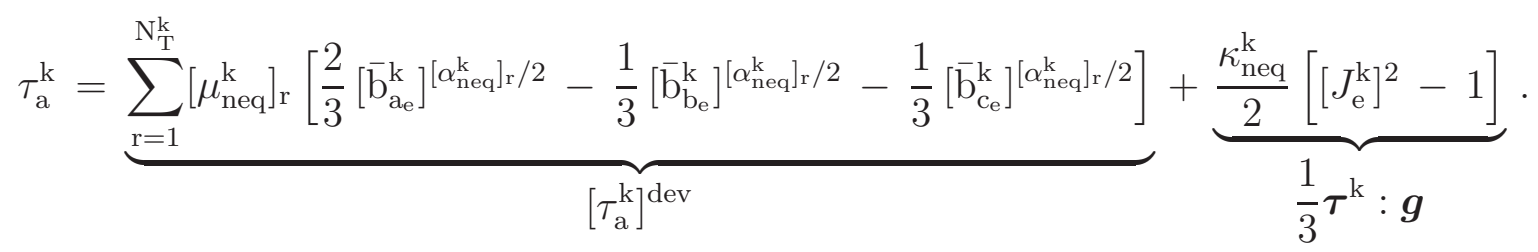

Note that $\mathrm{N}_{\mathrm{T}}$ and $\mathrm{N}_{\mathrm{T}}^{\mathrm{k}}$ are positive integers, $\left[\mu_{\mathrm{eq}}\right]_{\mathrm{r}}$ and $\left[\mu_{\mathrm{neq}}^{\mathrm{k}}\right]_{\mathrm{r}}$ are constant shear moduli, and $\left[\alpha_{\mathrm{eq}}\right]_{\mathrm{r}}$ and $\left[\alpha_{\text {neq }}^{\mathrm{k}}\right]_{\mathrm{r}}$ are dimensionless constants.

With the material parameters $\hat{\tau}^{\mathrm{k}}$ as relaxation times, the deviatoric and volumetric viscosities $\eta_{\mathrm{D}}^{\mathrm{k}}$ and $\eta_{\mathrm{v}}^{\mathrm{k}}$ - introduced in equation 3.37 - are calculated by

$$
\eta_{\mathrm{D}}^{\mathrm{k}}=\hat{\tau}^{\mathrm{k}} \sum_{\mathrm{r}=1}^{\mathrm{N}_{\mathrm{T}}^{\mathrm{k}}}\left[\mu_{\mathrm{neq}}^{\mathrm{k}}\right]_{\mathrm{r}}\left[\alpha_{\mathrm{neq}}^{\mathrm{k}}\right]_{\mathrm{r}}
$$

and

$$
\eta_{\mathrm{v}}^{\mathrm{k}}=\hat{\tau}^{\mathrm{k}} \kappa_{\mathrm{neq}}^{\mathrm{k}}
$$

For the calculation of the matrix $K_{\mathrm{ab}}^{\mathrm{k}}$ needed in the local Newton-iteration it remains to determine the derivatives $\left(\partial\left[\tau_{\mathrm{a}}^{\mathrm{k}}\right]^{\operatorname{dev}}\right) /\left(\partial \varepsilon_{\mathrm{b}_{\mathrm{e}}}^{\mathrm{k}}\right)$ and $\left(\partial \boldsymbol{\tau}^{\mathrm{k}}: \boldsymbol{g}\right) /\left(\partial \varepsilon_{\mathrm{b}_{\mathrm{e}}}^{\mathrm{k}}\right)$ for the used Ogden type free energy function. Taking into account that

$$
\begin{aligned}
& \frac{\partial \overline{\mathrm{b}}_{a_{\mathrm{e}}}^{\mathrm{k}}}{\partial \varepsilon_{\mathrm{a}_{\mathrm{e}}}^{\mathrm{k}}}=\frac{\partial \overline{\mathrm{b}}_{\mathrm{a}_{\mathrm{e}}^{\mathrm{k}}}^{\mathrm{k}}}{\partial \lambda_{\mathrm{a}_{\mathrm{e}}}^{\mathrm{k}}} \lambda_{\mathrm{a}_{\mathrm{e}}}^{\mathrm{k}}=\frac{4}{3} \overline{\mathrm{b}}_{a_{\mathrm{e}}}^{\mathrm{k}}, \\
& \frac{\partial \overline{\mathrm{b}}_{a_{\mathrm{e}}}^{\mathrm{k}}}{\partial \varepsilon_{\mathrm{b}_{\mathrm{e}}}^{\mathrm{k}}}=\frac{\partial \overline{\mathrm{b}}_{a_{\mathrm{e}}}^{\mathrm{k}}}{\partial \lambda_{\mathrm{b}_{\mathrm{e}}}^{\mathrm{k}}} \lambda_{\mathrm{b}_{\mathrm{e}}}^{\mathrm{k}}=-\frac{2}{3} \overline{\mathrm{b}}_{a_{\mathrm{e}}}^{\mathrm{k}}, \quad(\mathrm{a} \neq \mathrm{b}), \\
& \frac{\partial J_{\mathrm{e}}^{\mathrm{k}}}{\partial \varepsilon_{\mathrm{a}_{\mathrm{e}}}^{\mathrm{k}}}=J_{\mathrm{e}}^{\mathrm{k}},
\end{aligned}
$$

for the applied Ogden material law these derivatives yield $(a \neq b, a \neq c, b \neq c)$

$$
\frac{\partial\left[\tau_{\mathrm{a}}^{\mathrm{k}}\right]^{\mathrm{dev}}}{\partial \varepsilon_{\mathrm{a}_{\mathrm{e}}}^{\mathrm{k}}}=\sum_{\mathrm{r}=1}^{\mathrm{N}_{\mathrm{T}}^{\mathrm{k}}}\left[\mu_{\mathrm{neq}}^{\mathrm{k}}\right]_{\mathrm{r}}\left[\alpha_{\text {neq }}^{\mathrm{k}}\right]_{\mathrm{r}}\left[\frac{4}{9}\left[\overline{\mathrm{b}}_{\mathrm{a}_{\mathrm{e}}}^{\mathrm{k}}\right]^{\left[\alpha_{\mathrm{neq}}^{\mathrm{k}}\right]_{\mathrm{r}} / 2}+\frac{1}{9}\left[\overline{\mathrm{b}}_{\mathrm{b}_{\mathrm{e}}}^{\mathrm{k}}\right]^{\left[\alpha_{\text {neq }}^{\mathrm{k}}\right]_{\mathrm{r}} / 2}+\frac{1}{9}\left[\overline{\mathrm{b}}_{\mathrm{ce}_{\mathrm{e}}}^{\mathrm{k}}\right]^{\left[\alpha_{\text {neq }}^{\mathrm{k}}\right]_{\mathrm{r}} / 2}\right],
$$




$$
\begin{aligned}
& \frac{\partial\left[\tau_{\mathrm{a}}^{\mathrm{k}}\right]^{\mathrm{dev}}}{\partial \varepsilon_{\mathrm{b}_{\mathrm{e}}}^{\mathrm{k}}}=\sum_{\mathrm{r}=1}^{\mathrm{N}_{\mathrm{T}}^{\mathrm{k}}}\left[\mu_{\mathrm{neq}}^{\mathrm{k}}\right]_{\mathrm{r}}\left[\alpha_{\mathrm{neq}}^{\mathrm{k}}\right]_{\mathrm{r}}\left[-\frac{2}{9}\left[\overline{\mathrm{b}}_{\mathrm{a}_{\mathrm{e}}}^{\mathrm{k}}\right]^{\left[\alpha_{\text {neq }}^{\mathrm{k}}\right]_{\mathrm{r}} / 2}-\frac{2}{9}\left[\overline{\mathrm{b}}_{\mathrm{b}_{\mathrm{e}}}^{\mathrm{k}}\right]^{\left[\alpha_{\text {neq }}^{\mathrm{k}}\right]_{\mathrm{r}} / 2}+\frac{1}{9}\left[\overline{\mathrm{b}}_{\mathrm{c}_{\mathrm{e}}}^{\mathrm{k}}\right]^{\left[\alpha_{\mathrm{neq}}^{\mathrm{k}}\right]_{\mathrm{r}} / 2}\right], \\
& \frac{\partial\left(\boldsymbol{\tau}^{\mathrm{k}}: \boldsymbol{g}\right)}{\partial \varepsilon_{\mathrm{a} \mathrm{e}}^{\mathrm{k}}}=3 \kappa_{\mathrm{neq}}^{\mathrm{k}}\left[J_{\mathrm{e}}^{\mathrm{k}}\right]^{2}
\end{aligned}
$$

\subsubsection{Tangent operator}

The application of a nonlinear material law in the context of a Finite Element formulation requires the derivation of a material tangent operator used in a global Newton-Raphson iteration algorithm (see, e.g. Ogden [59], Hughes [26], and Zienkewicz and Taylor [76]). In the spatial configuration the total tangent operator conjugate to the Kirchhoff stresses is defined by

$$
\mathbf{c}=\mathbf{c}^{\mathrm{eq}}+\sum_{\mathrm{k}=1}^{\mathrm{N}} \mathbf{c}^{\mathrm{k}}
$$

In the following this operator is computed for the isotropic tensor functions $\Psi^{\mathrm{eq}}$ and $\Psi^{\mathrm{k}}$ presented in equations (3.60) and (3.62), respectively.

\section{Equilibrium tangent operator}

For the material formulation the equilibrium term yields (see Ogden [59] or Holzapfel [23])

$$
\begin{aligned}
\mathbf{C}^{\mathrm{eq}}= & 2 \frac{\partial \boldsymbol{S}^{\mathrm{eq}}}{\partial \boldsymbol{C}}=4 \frac{\partial^{2} \Psi^{\mathrm{eq}}}{\partial \boldsymbol{C} \otimes \partial \boldsymbol{C}} \\
= & \sum_{\mathrm{a}, \mathrm{b}=1}^{3} \frac{1}{\lambda_{\mathrm{b}}} \frac{\partial S_{\mathrm{a}}^{\mathrm{eq}}}{\partial \lambda_{\mathrm{b}}} \boldsymbol{N}_{\mathrm{a}} \otimes \boldsymbol{N}_{\mathrm{a}} \otimes \boldsymbol{N}_{\mathrm{b}} \otimes \boldsymbol{N}_{\mathrm{b}} \\
& +\sum_{\substack{\mathrm{a}, \mathrm{b}=1 \\
\mathrm{a} \neq \mathrm{b}}}^{3} \frac{S_{\mathrm{b}}^{\mathrm{eq}}-S_{\mathrm{a}}^{\mathrm{eq}}}{\lambda_{\mathrm{b}}^{2}-\lambda_{\mathrm{a}}^{2}} \boldsymbol{N}_{\mathrm{a}} \otimes \boldsymbol{N}_{\mathrm{b}} \otimes\left[\boldsymbol{N}_{\mathrm{a}} \otimes \boldsymbol{N}_{\mathrm{b}}+\boldsymbol{N}_{\mathrm{b}} \otimes \boldsymbol{N}_{\mathrm{a}}\right]
\end{aligned}
$$

whereby $S_{\mathrm{a}}^{\mathrm{eq}}$ are the principal stresses of the second Piola-Kirchhoff stress tensor $\boldsymbol{S}^{\mathrm{eq}}$ with

$$
\boldsymbol{S}^{\mathrm{eq}}=\sum_{\mathrm{a}=1}^{3} \frac{1}{\lambda_{\mathrm{a}}} \frac{\partial \Psi^{\mathrm{eq}}}{\partial \lambda_{\mathrm{a}}} \boldsymbol{N}_{\mathrm{a}} \otimes \boldsymbol{N}_{\mathrm{a}}=\sum_{\mathrm{a}=1}^{3} S_{\mathrm{a}}^{\mathrm{eq}} \boldsymbol{N}_{\mathrm{a}} \otimes \boldsymbol{N}_{\mathrm{a}}
$$

and $\boldsymbol{N}_{\mathrm{a}}$ is the set of orthogonal eigenvectors of the right Cauchy-Green tensor (see equation (2.30)). By isotropy it follows that if two or all three eigenvalues $\lambda_{\text {a }}^{2}$ of $\boldsymbol{C}$ (and thus also of $\boldsymbol{b}$ ) are equal, the corresponding principal stresses $S_{\mathrm{a}}^{\mathrm{eq}}$ are equal, too. For these cases the application of l'Hôpital's rule on the difference $\left[S_{\mathrm{b}}^{\mathrm{eq}}-S_{\mathrm{a}}^{\mathrm{eq}}\right] /\left[\lambda_{\mathrm{b}}^{2}-\lambda_{\mathrm{a}}^{2}\right]$ in equation 
(3.74) yields ${ }^{2}$

$$
\lim _{\lambda_{\mathrm{b}} \rightarrow \lambda_{\mathrm{a}}} \frac{S_{\mathrm{b}}^{\mathrm{eq}}-S_{\mathrm{a}}^{\mathrm{eq}}}{\lambda_{\mathrm{b}}^{2}-\lambda_{\mathrm{a}}^{2}}=\frac{\partial S_{\mathrm{b}}^{\mathrm{eq}}}{\partial \lambda_{\mathrm{b}}^{2}}-\frac{\partial S_{\mathrm{a}}^{\mathrm{eq}}}{\partial \lambda_{\mathrm{b}}^{2}} .
$$

Thereby, also for the cases $\lambda_{1} \neq \lambda_{2}=\lambda_{3}$ and $\lambda_{1}=\lambda_{2}=\lambda_{3}$ the tangent operator is defined properly. Pushforward operation renders the spatial equilibrium tangent operator c $^{\text {eq }}$ with

$$
\begin{aligned}
\mathbf{c}^{\mathrm{eq}} & =\boldsymbol{\varphi}_{*} \mathbf{C}^{\mathrm{eq}} \\
& =\sum_{\mathrm{a}, \mathrm{b}=1}^{3} \lambda_{\mathrm{a}}^{2} \lambda_{\mathrm{b}} \frac{\partial S_{\mathrm{a}}^{\mathrm{eq}}}{\partial \lambda_{\mathrm{b}}} \boldsymbol{n}_{\mathrm{a}} \otimes \boldsymbol{n}_{\mathrm{a}} \otimes \boldsymbol{n}_{\mathrm{b}} \otimes \boldsymbol{n}_{\mathrm{b}} \\
& +\sum_{\substack{\mathrm{a}, \mathrm{b}=1 \\
\mathrm{a} \neq \mathrm{b}}}^{3} \frac{\tau_{\mathrm{b}}^{\mathrm{eq}} \lambda_{\mathrm{a}}^{2}-\tau_{\mathrm{a}}^{\mathrm{eq}} \lambda_{\mathrm{b}}^{2}}{\lambda_{\mathrm{b}}^{2}-\lambda_{\mathrm{a}}^{2}} \boldsymbol{n}_{\mathrm{a}} \otimes \boldsymbol{n}_{\mathrm{b}} \otimes\left[\boldsymbol{n}_{\mathrm{a}} \otimes \boldsymbol{n}_{\mathrm{b}}+\boldsymbol{n}_{\mathrm{b}} \otimes \boldsymbol{n}_{\mathrm{a}}\right]
\end{aligned}
$$

The principal Kirchhoff stresses $\tau_{\mathrm{a}}$ is described in equation (3.43) and $\boldsymbol{n}_{\mathrm{a}}$ is the corresponding set of normed eigenvectors. Also here a consideration of the limit of the difference $\left[\tau_{\mathrm{b}} \lambda_{\mathrm{a}}^{2}-\tau_{\mathrm{a}} \lambda_{\mathrm{b}}^{2}\right] /\left[\lambda_{\mathrm{b}}^{2}-\lambda_{\mathrm{a}}^{2}\right]$ for $\lambda_{\mathrm{a}}=\lambda_{\mathrm{b}}$ for $\lambda_{\mathrm{a}}=\lambda_{\mathrm{b}}$ has to be considered. The application of l'Hôpital's rule yields

$$
\lim _{\lambda_{\mathrm{b}} \rightarrow \lambda_{\mathrm{a}}} \frac{\tau_{\mathrm{b}}^{\mathrm{eq}} \lambda_{\mathrm{a}}^{2}-\tau_{\mathrm{a}}^{\mathrm{eq}} \lambda_{\mathrm{b}}^{2}}{\lambda_{\mathrm{b}}^{2}-\lambda_{\mathrm{a}}^{2}}=\frac{1}{2} \lambda_{\mathrm{a}}\left[\frac{\partial \tau_{\mathrm{b}}^{\mathrm{eq}}}{\partial \lambda_{\mathrm{b}}}-\frac{\partial \tau_{\mathrm{a}}^{\mathrm{eq}}}{\partial \lambda_{\mathrm{b}}}\right]-\tau_{\mathrm{a}}^{\mathrm{eq}}
$$

\section{Non-equilibrium tangent operator}

For the calculation of the non-equilibrium part of the tangent operator (see Reese and Govindjee [65])

$$
\mathbf{c}^{\mathrm{k}}=\boldsymbol{\varphi}_{*} \mathbf{C}^{\mathrm{k}}=\boldsymbol{\varphi}_{*}\left(2 \frac{\partial \boldsymbol{S}^{\mathrm{k}}}{\partial \boldsymbol{C}}\right)=\boldsymbol{\varphi}_{*}\left(4 \frac{\partial^{2} \Psi^{\mathrm{k}}}{\partial \boldsymbol{C} \otimes \partial \boldsymbol{C}}\right)
$$

the stress tensor

$$
\widetilde{\boldsymbol{S}}^{\mathrm{k}}=\left[\boldsymbol{F}_{\mathrm{e}}^{\mathrm{k}}\right]_{\mathrm{tr}}^{-1} \cdot \boldsymbol{\tau}^{\mathrm{k}} \cdot\left[\boldsymbol{F}_{\mathrm{e}}^{\mathrm{k}}\right]_{\mathrm{tr}}^{-\mathrm{T}}=\sum_{\mathrm{a}=1}^{3} \underbrace{\frac{\tau_{\mathrm{a}}^{\mathrm{k}}}{\left[\lambda_{\mathrm{a}}^{\mathrm{k}}\right]_{\mathrm{tr}}^{2}}}_{\widetilde{S}_{\mathrm{a}}^{\mathrm{k}}} \widetilde{\boldsymbol{N}}_{\mathrm{a}}^{\mathrm{k}} \otimes \widetilde{\boldsymbol{N}}_{\mathrm{a}}^{\mathrm{k}}
$$

is introduced. Also the evaluation of the modulus

$$
\left[C_{\mathrm{ac}}^{\mathrm{alg}}\right]^{\mathrm{k}}=\sum_{\mathrm{b}=1}^{3} \frac{\partial \tau_{\mathrm{a}}^{\mathrm{k}}}{\partial \varepsilon_{\mathrm{b}_{\mathrm{e}}}^{\mathrm{k}}}\left[K_{\mathrm{bc}}^{\mathrm{k}}\right]^{-1}=\sum_{\mathrm{b}=1}^{3} \frac{\partial\left(\left[\tau_{\mathrm{a}}^{\mathrm{k}}\right]^{\mathrm{dev}}+\frac{1}{3} \boldsymbol{\tau}^{\mathrm{k}}: \boldsymbol{g}\right)}{\partial \varepsilon_{\mathrm{b}_{\mathrm{e}}}^{\mathrm{k}}}\left[K_{\mathrm{bc}}^{\mathrm{k}}\right]^{-1}
$$

${ }^{2}$ The application of l'Hôpital's rule yields

$$
\begin{aligned}
\lim _{\lambda_{\mathrm{b}} \rightarrow \lambda_{\mathrm{a}}} \frac{S_{\mathrm{b}}^{\mathrm{eq}}-S_{\mathrm{a}}^{\mathrm{eq}}}{\lambda_{\mathrm{b}}^{2}-\lambda_{\mathrm{a}}^{2}} & =\lim _{\lambda_{\mathrm{b}} \rightarrow \lambda_{\mathrm{a}}} \frac{\frac{\partial}{\partial \lambda_{\mathrm{b}}}\left(S_{\mathrm{b}}^{\mathrm{eq}}-S_{\mathrm{a}}^{\mathrm{eq}}\right)}{\frac{\partial}{\partial \lambda_{\mathrm{b}}}\left(\lambda_{\mathrm{b}}^{2}-\lambda_{\mathrm{a}}^{2}\right)} \\
& =\left[\frac{\partial S_{\mathrm{b}}^{\mathrm{eq}}}{\partial \lambda_{\mathrm{b}}}-\frac{\partial S_{\mathrm{a}}^{\mathrm{eq}}}{\partial \lambda_{\mathrm{b}}}\right] \frac{1}{2 \lambda_{\mathrm{b}}}=\left[\frac{\partial S_{\mathrm{b}}^{\mathrm{eq}}}{\partial \lambda_{\mathrm{b}}}-\frac{\partial S_{\mathrm{a}}^{\mathrm{eq}}}{\partial \lambda_{\mathrm{b}}}\right] \frac{\partial \lambda_{\mathrm{b}}}{\partial \lambda_{\mathrm{b}}^{2}}=\frac{\partial S_{\mathrm{b}}^{\mathrm{eq}}}{\partial \lambda_{\mathrm{b}}^{2}}-\frac{\partial S_{\mathrm{a}}^{\mathrm{eq}}}{\partial \lambda_{\mathrm{b}}^{2}} .
\end{aligned}
$$


is required which is computed by taking into account equations (3.59) and (3.69)-(3.71). Pushforward transformation of

$$
\begin{aligned}
\widetilde{\mathbf{C}}^{\mathrm{k}}= & \sum_{\mathrm{a}=1}^{3} \sum_{\mathrm{b}=1}^{3}\left[\frac{1}{\left[\lambda_{\mathrm{a}_{\mathrm{e}}^{\mathrm{e}}}^{\mathrm{k}}\right]_{\mathrm{tr}}^{2}\left[\lambda_{\mathrm{b} \mathrm{e}}^{\mathrm{k}}\right]_{\mathrm{tr}}^{2}}\left[\left[C_{\mathrm{ab}}^{\mathrm{alg}}\right]^{\mathrm{k}}-\tau_{\mathrm{a}}^{\mathrm{k}} 2 \delta_{\mathrm{ab}}\right] \widetilde{\boldsymbol{N}}_{\mathrm{a}}^{\mathrm{k}} \otimes \widetilde{\boldsymbol{N}}_{\mathrm{a}}^{\mathrm{k}} \otimes \widetilde{\boldsymbol{N}}_{\mathrm{b}}^{\mathrm{k}} \otimes \widetilde{\boldsymbol{N}}_{\mathrm{b}}^{\mathrm{k}}\right] \\
& +\frac{1}{2} \sum_{\mathrm{a}=1}^{3} \sum_{\mathrm{b}=1}^{3} 2 \frac{\widetilde{S}_{\mathrm{b}}^{\mathrm{k}}-\widetilde{S}_{\mathrm{a}}^{\mathrm{k}}}{\left[\lambda_{\mathrm{b}_{\mathrm{e}}}^{\mathrm{k}}\right]_{\mathrm{tr}}^{2}-\left[\lambda_{\mathrm{a} \mathrm{e}}^{\mathrm{k}}\right]_{\mathrm{tr}}^{2}} \widetilde{\boldsymbol{N}}_{\mathrm{a}}^{\mathrm{k}} \otimes \widetilde{\boldsymbol{N}}_{\mathrm{b}}^{\mathrm{k}} \otimes\left[\widetilde{\boldsymbol{N}}_{\mathrm{a}}^{\mathrm{k}} \otimes \widetilde{\boldsymbol{N}}_{\mathrm{b}}^{\mathrm{k}}+\widetilde{\boldsymbol{N}}_{\mathrm{b}}^{\mathrm{k}} \otimes \widetilde{\boldsymbol{N}}_{\mathrm{a}}^{\mathrm{k}}\right] \\
= & \sum_{\mathrm{a}=1}^{3} \sum_{\mathrm{b}=1}^{3} \sum_{\mathrm{c}=1}^{3} \sum_{\mathrm{d}=1}^{3} \widetilde{L}_{\mathrm{abcd}}^{\mathrm{k}} \widetilde{\boldsymbol{N}}_{\mathrm{a}}^{\mathrm{k}} \otimes \widetilde{\boldsymbol{N}}_{\mathrm{b}}^{\mathrm{k}} \otimes \widetilde{\boldsymbol{N}}_{\mathrm{c}}^{\mathrm{k}} \otimes \widetilde{\boldsymbol{N}}_{\mathrm{d}}^{\mathrm{k}}
\end{aligned}
$$

with respect to $\left[\boldsymbol{F}_{\mathrm{e}}^{\mathrm{k}}\right]_{\text {tr }}$ renders the non-equilibrium part of the tangent operator

$$
\mathbf{c}^{\mathrm{k}}=\sum_{\mathrm{a}=1}^{3} \sum_{\mathrm{b}=1}^{3} \sum_{\mathrm{c}=1}^{3} \sum_{\mathrm{d}=1}^{3} \underbrace{\widetilde{L}_{\mathrm{abcd}}^{\mathrm{k}}\left[\lambda_{\mathrm{a}_{\mathrm{e}}}^{\mathrm{k}}\right]_{\mathrm{tr}}\left[\lambda_{\mathrm{b}_{\mathrm{e}}}^{\mathrm{k}}\right]_{\mathrm{tr}}\left[\lambda_{\mathrm{c}_{\mathrm{e}}}^{\mathrm{k}}\right]_{\mathrm{tr}}\left[\lambda_{\mathrm{d}_{\mathrm{e}}}^{\mathrm{k}}\right]_{\mathrm{tr}}}_{\mathrm{c}_{\mathrm{abcd}}^{\mathrm{k}}} \boldsymbol{n}_{\mathrm{a}} \otimes \boldsymbol{n}_{\mathrm{b}} \otimes \boldsymbol{n}_{\mathrm{c}} \otimes \boldsymbol{n}_{\mathrm{d}}
$$

The limit of the divided difference in equation 3.83 is

$$
\lim _{\lambda_{\mathrm{b}} \rightarrow \lambda_{\mathrm{a}}} \frac{\widetilde{S}_{\mathrm{b}}^{\mathrm{k}}-\widetilde{S}_{\mathrm{a}}^{\mathrm{k}}}{\left[\lambda_{\mathrm{b}_{\mathrm{e}}}^{\mathrm{k}}\right]_{\mathrm{tr}}^{2}-\left[\lambda_{\mathrm{a}_{\mathrm{e}}}^{\mathrm{k}}\right]_{\mathrm{tr}}^{2}}=\frac{\partial \widetilde{S}_{\mathrm{b}}^{\mathrm{k}}}{\partial\left[\lambda_{\mathrm{b}_{\mathrm{e}}}^{\mathrm{k}}\right]_{\mathrm{tr}}^{2}}-\frac{\partial \widetilde{S}_{\mathrm{a}}^{\mathrm{k}}}{\partial\left[\lambda_{\mathrm{b}_{\mathrm{e}}}^{\mathrm{k}}\right]_{\mathrm{tr}}^{2}} .
$$




\section{Basic methods for parameter identification}

For the simulation of the deformation behavior of materials and structures in engineering applications, reliable material models and the corresponding material parameters have to be determined. The different steps within the strategy to develop simulation techniques are (see Mahnken [43]): First, a model concept is formed which is based on observations from experiments made in correspondence to the respective engineering application. Hereby the basic phenomena are characterized and the observation scale for the material model is determined. Then the constitutive model is formulated. The parameter identification based on the comparison of simulated and experimentally measured data follows. With a verification the quality of the model and accordingly the ability to simulate the experimental data from the laboratory tests used for the parameter identification is analyzed. In order to check if the material model and the identified parameters are able to simulate also experimental data from other tests made under different conditions, a validation is made finally. Thus, by validation the simulated data is compared with experimental which has not been used for the parameter identification.

The basic methods within a parameter identification process are described in this chapter. The inverse problem is formulated in section 4.1. In section 4.2 two methods for optical measurements are introduced. The interpolation of the measured displacement fields is described in subsection 4.3. In section 4.4 the methods, which are used for the numerical solution of the inverse problem in this work, are presented. In the end in section 4.5 stability problems - usually occurring during the identification process - are investigated.

\subsection{The inverse problem}

The constitutive equations for the viscoelastic material law - as presented in chapter 3 - deliver the functional relationship between the stresses and the strains. The solution of this boundary value problem (BVP) is termed the direct problem. Considering the viscoelastic material law as a model function $g(\boldsymbol{\kappa}, \boldsymbol{f}, \boldsymbol{u})$ which defines the output variable $\boldsymbol{u}$ for given input variables $\boldsymbol{f}$ and material parameters $\boldsymbol{\kappa}$, the direct problem yields (see e.g. Mahnken [43]):

$$
\text { find } \boldsymbol{u}^{*} \text {, such that } g(\boldsymbol{\kappa}, \boldsymbol{f}, \boldsymbol{u})=0 \text { for given } \boldsymbol{f} \text { and } \boldsymbol{\kappa} \text {. }
$$

For the parameter identification of the model function the corresponding inverse problem is defined as (see e.g. Banks and Kunisch [2], Mahnken [43] and Bui [13])

$$
\text { find } \boldsymbol{\kappa}^{*} \text {, such that } g(\boldsymbol{\kappa}, \boldsymbol{f}, \boldsymbol{u})=0 \text { for given } \boldsymbol{f} \text { and } \boldsymbol{u} \text {. }
$$


The aim of the parameter identification and accordingly the solution of the inverse problem for the viscoelastic material law is to optimize the material parameters so that the material model delivers simulation data which optimally agree with the experimental data. In this work the simulation data and experimental data which are compared are displacement fields. By variation of the material parameters the displacement fields are varied, whereby for the simulation the geometry of the specimen and the boundary conditions including the load forces are chosen in analogy to the experiment. For the identification process the experimentally measured displacement fields have to be made comparable to the FEM results. Therefore, the measured displacements of the specimen are interpolated on chosen identification nodes of the FE model (see section 4.3).

\subsection{Non-contacting optical strain measurement systems}

The systems applied for the displacement measurements in this work are the image correlation photogrammetry and the video extensometry. Both are non-contacting optical measurement methods which therefore have no influence on the deformation behavior of the specimen and consequently are appropriate for the displacement measurements of soft polymers. Another advantage is the high accuracy of these systems.

\subsubsection{Image correlation photogrammetry}

The principle of image correlation photogrammetry bases on the comparison of digitized images, taken in the reference configuration and the deformed configurations of a specimen in a test. For the application first a black and white stochastic pattern is applied onto the specimen's surface. Using (one or) two CCD cameras (see figure 4.1) the system tracks this pattern with sub-pixel accuracy during the tests. Therefore synchronized stereo images of the pattern are recorded at different load stages. After acquisition the images are digitized and stored for analyses.

The three-dimensional displacement fields are evaluated by using a photogrammetric evaluation procedure (ARAMIS ${ }^{1}$ ). Thereby, the images are characterized by the patterns of different levels of light intensity. The processed image for the initial step defines a set of so-called macroimage facets which are tracked in the following images with sub-pixel accuracy. The evaluation procedure calculates the two- or three dimensional coordinates of the surface of the specimen, which are related to the facets at each load step.

For details to this measurement method see, e.g. Winter [74].

\subsubsection{Video extensometry}

The method of video extensometry measures displacements by tracking contrasting gauge marks placed on the specimen. The mono and synchronized stereo images taken by one or two CCD cameras are analyzed using image processing algorithms that track the center of the marks.

\footnotetext{
${ }^{1}$ Version 5.4 (Gesellschaft für optische Messtechnik mbH)
} 


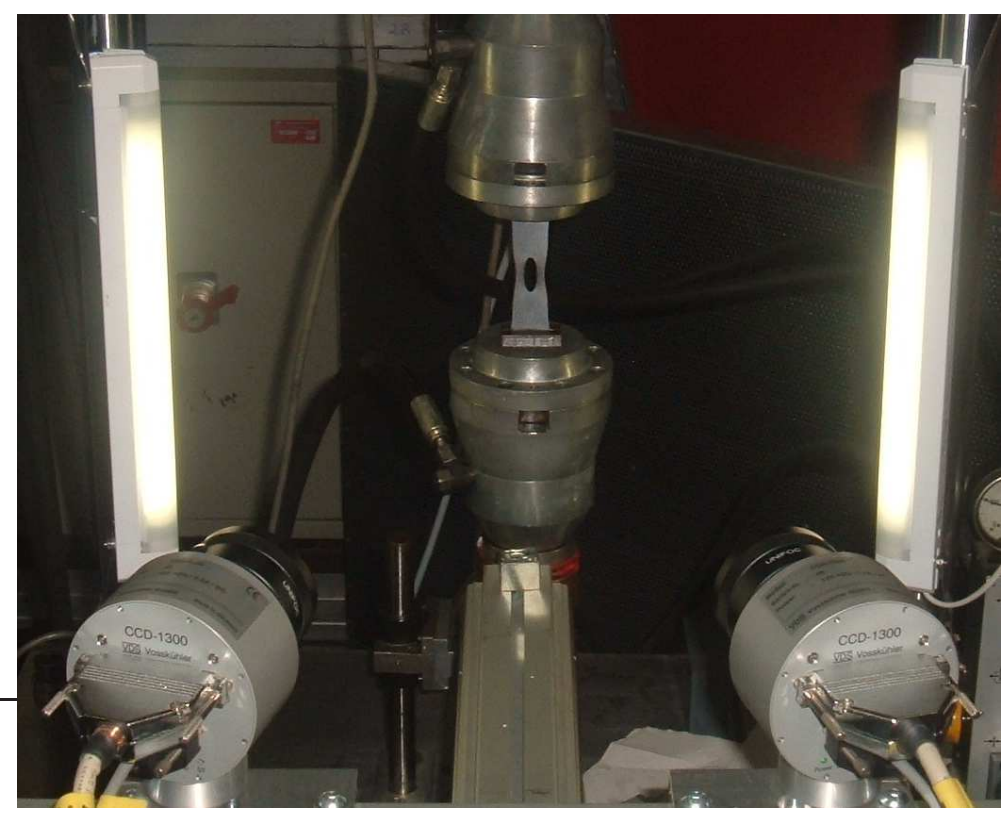

Figure 4.1: $C C D$ cameras for image correlation photogrammetry in a tensile test.

\subsection{Interpolation of experimental data}

As mentioned above the simulated displacements must be comparable to the experimentally determined displacements which means that the coordinates of the identification nodes for the reference configuration have to coincide with the respective coordinates of the measurement points which is not the case in general (see e.g. Banks and Kunisch [2]). Therefore, with an interpolation rule which satisfies the geometry of the respective specimen, the experimentally measured displacements have to be mapped onto the coordinates of the identification nodes. In this section the basic techniques for the interpolation of experimental data for parameter identification are described.

Remark 4.3.1 Note that the interpolation routines must be conducted with most possible precision. Due to the general ill-posedness of the inverse problem (see section 4.5) even small inaccuracies within the interpolation procedure will have a strong influence on the solution and can easily lead to divergence within the parameter identification iteration. On the one hand the measurement data has to be chosen carefully. I.e. in some cases some of the measurement points are scattered due to uncertainties at the boundaries of the specimen. Only without taking into account these measurement points, the later identification procedure can deliver a reliable parameter set. In addition, the interpolation of the measured displacements on the coordinates of the identification nodes has to be admissible. Therefore, the measurement points have to be chosen in an appropriate neighborhood to the respective identification node. Generally, extrapolations are not admissible.

\subsubsection{One-dimensional interpolation}

For the example of a cylinder with the radius $d$ - which is straight in the reference configuration - the one-dimensional interpolation of two-dimensional and three-dimensional 
displacement data is described. The measurement points and identification nodes lie on a straight line along the exterior of the cylinder. The piecewise linear and quadratic interpolation of the experimentally measured displacements on the identification nodes are considered. For the linear interpolation the two nearest, for the quadratic interpolation the three nearest measurement points are used. Figure 4.2 shows an example for the linear and quadratic interpolation of two-dimensional measurement data, whereas in figure 4.3 the linear and quadratic interpolation of three-dimensional measurement data is presented.

\section{Linear one-dimensional interpolation}

Let $(x, y=d)$ be the coordinates of an identification node in the reference configuration and let $\left(x_{1}, y_{1}=d\right)$ and $\left(x_{2}, y_{2}=d\right)$ be the coordinates of the two nearest measurement points in the reference configuration. Moreover, $\boldsymbol{D}_{1}^{\exp }$ and $\boldsymbol{D}_{2}^{\exp }$ are the displacement vectors associated to the measurement points. The displacement vector $\boldsymbol{D}_{\text {proj }}^{\text {exp }}(x)$, being associated to the identification node, is calculated by linear interpolation using

$$
\boldsymbol{D}_{p r o j}^{e x p}(x)=\frac{x_{2}-x}{x_{2}-x_{1}} \boldsymbol{D}_{1}^{e x p}+\frac{x-x_{1}}{x_{2}-x_{1}} \boldsymbol{D}_{2}^{e x p} .
$$

\section{Quadratic one-dimensional interpolation}

Let $(x, y=d)$ be the coordinates of an identification node in the reference configuration and let $\left(x_{1}, y_{1}=d\right),\left(x_{2}, y_{2}=d\right)$, and $\left(x_{3}, y_{3}=d\right)$ be the coordinates of the three nearest measurement points in the reference configuration. Moreover, $\boldsymbol{D}_{1}^{\exp }, \boldsymbol{D}_{2}^{\exp }$, and $\boldsymbol{D}_{3}^{e x p}$ are the displacement vectors associated to the measurement points. Therefore, the interpolated displacement vector $\boldsymbol{D}_{p r o j}^{e x p}(x)$, which is associated to the identification node, yields

$$
\begin{aligned}
\boldsymbol{D}_{\text {proj }}^{\text {exp }}(x)= & \frac{\left[x-x_{2}\right]\left[x-x_{3}\right]}{\left[x_{1}-x_{2}\right]\left[x_{1}-x_{3}\right]} \boldsymbol{D}_{1}^{\text {exp }}+\frac{\left[x-x_{1}\right]\left[x-x_{3}\right]}{\left[x_{2}-x_{1}\right]\left[x_{2}-x_{3}\right]} \boldsymbol{D}_{2}^{\text {exp }} \\
+ & \frac{\left[x-x_{1}\right]\left[x-x_{2}\right]}{\left[x_{3}-x_{1}\right]\left[x_{3}-x_{2}\right]} \boldsymbol{D}_{3}^{\text {exp }} .
\end{aligned}
$$



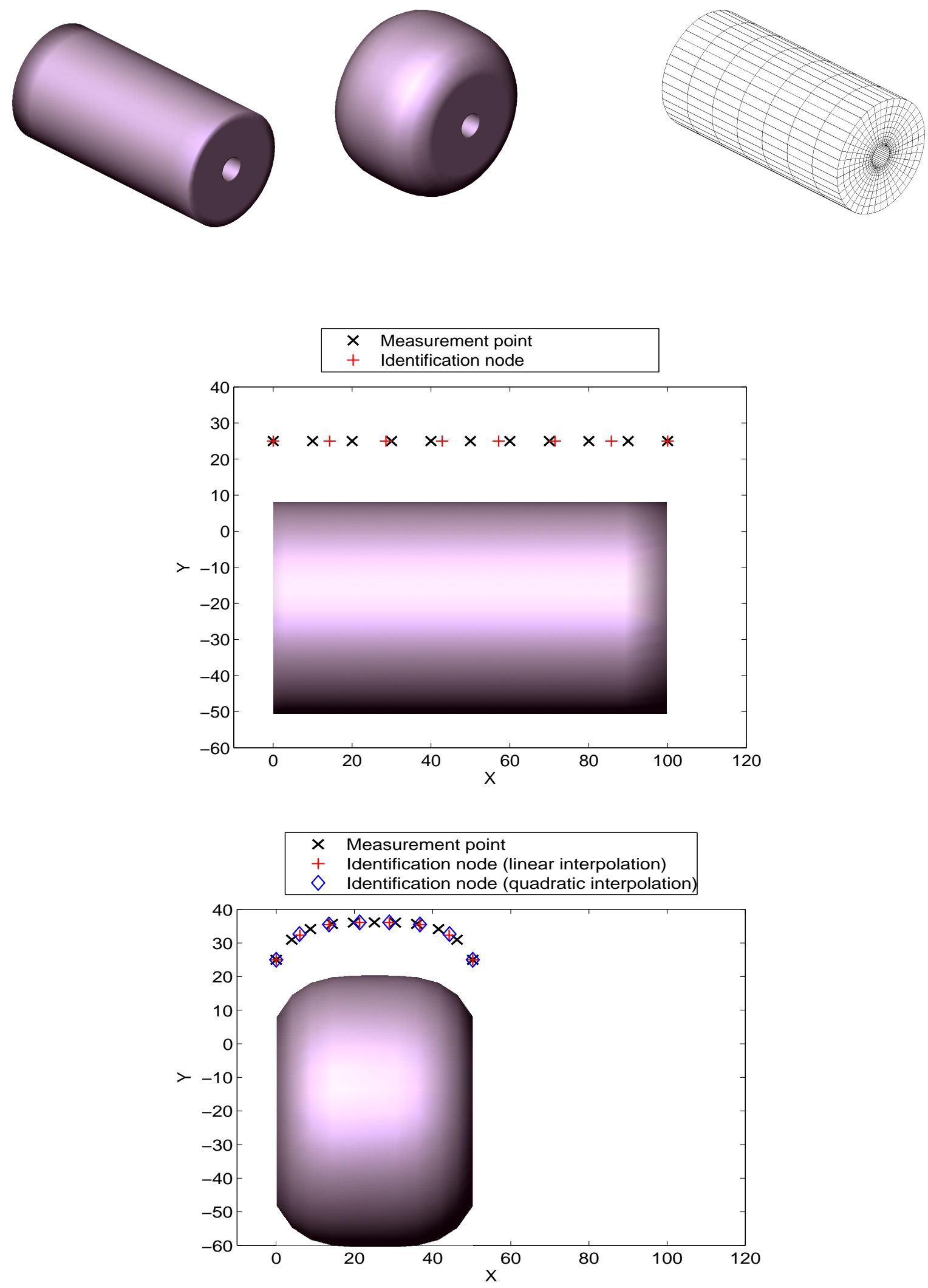

Figure 4.2: Example of a cylinder with two-dimensional measured displacements. Top: specimen in the reference configuration, specimen in the deformed configuration, and discretization of an associated FE model. Bottom: Interpolation of the displacements of the measurement points on the coordinates of the identification nodes. 

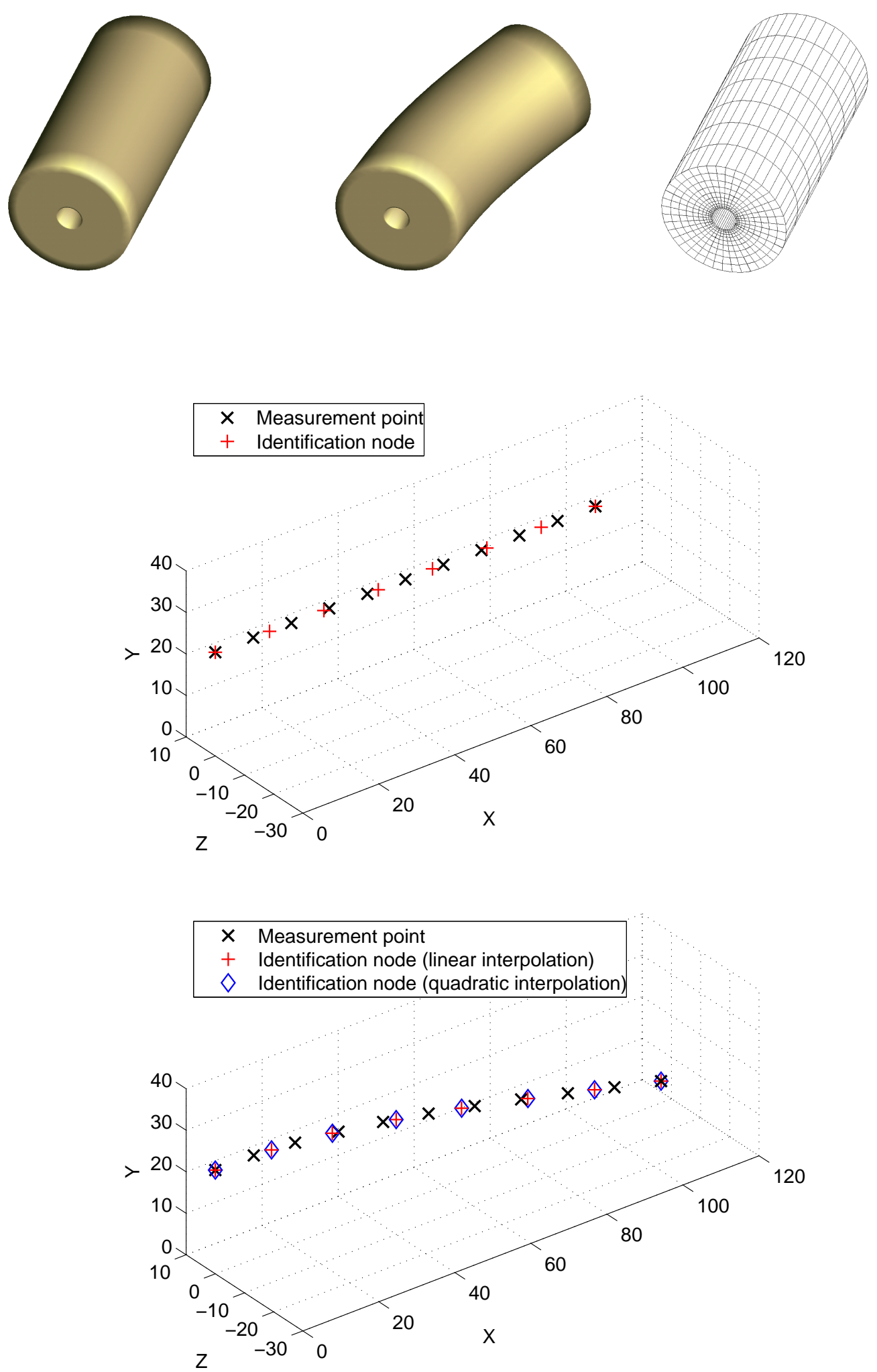

Figure 4.3: Example of a cylinder with three-dimensional measured displacements. Top: specimen in the reference configuration, specimen in the deformed configuration, and discretization of an associated FE model. Bottom: Interpolation of the displacements of the measurement points on the coordinates of the identification nodes. 


\subsubsection{Two-dimensional interpolation}

In this subsection the two-dimensional interpolation of measurement data is described. Considering the reference configuration, the measurement points and identification nodes lie on a plane. The examples in fig 4.4 and 4.5 show the interpolations of measured two-dimensional and three-dimensional displacement fields, respectively.

Within the interpolation algorithm the measured displacements are interpolated linearly on the identification nodes by triangulation (see, e.g. Scheday [66]). Let $(x, y)$ be the coordinates of an identification node in the reference configuration and $\left(x_{1}, y_{1}\right),\left(x_{2}, y_{2}\right)$, and $\left(x_{3}, y_{3}\right)$ the coordinates of the three nearest measurement points - which do not lie on a straight line - in the reference configuration. Let also $\boldsymbol{D}_{1}^{\exp }, \boldsymbol{D}_{2}^{\exp }$, and $\boldsymbol{D}_{3}^{\exp }$ be the displacement vectors associated to the measurement points. Therefore, the interpolated displacement vector $\boldsymbol{D}_{\text {proj }}^{e x p}(u, v)$, which is associated to the identification node, yields

$$
\boldsymbol{D}_{p r o j}^{e x p}(u, v)=[1-u-v] \boldsymbol{D}_{1}^{e x p}+u \boldsymbol{D}_{2}^{e x p}+v \boldsymbol{D}_{3}^{e x p},
$$

with

$$
u=\frac{\left[y_{3}-y_{1}\right]\left[x-x_{1}\right]-\left[x_{3}-x_{1}\right]\left[y-y_{1}\right]}{\left[y_{3}-y_{1}\right]\left[x_{2}-x_{1}\right]-\left[x_{3}-x_{1}\right]\left[y_{2}-y_{1}\right]}
$$

and

$$
v=\frac{\left[y_{1}-y_{2}\right]\left[x-x_{1}\right]+\left[x_{2}-x_{1}\right]\left[y-y_{1}\right]}{\left[y_{3}-y_{1}\right]\left[x_{2}-x_{1}\right]-\left[x_{3}-x_{1}\right]\left[y_{2}-y_{1}\right]}
$$



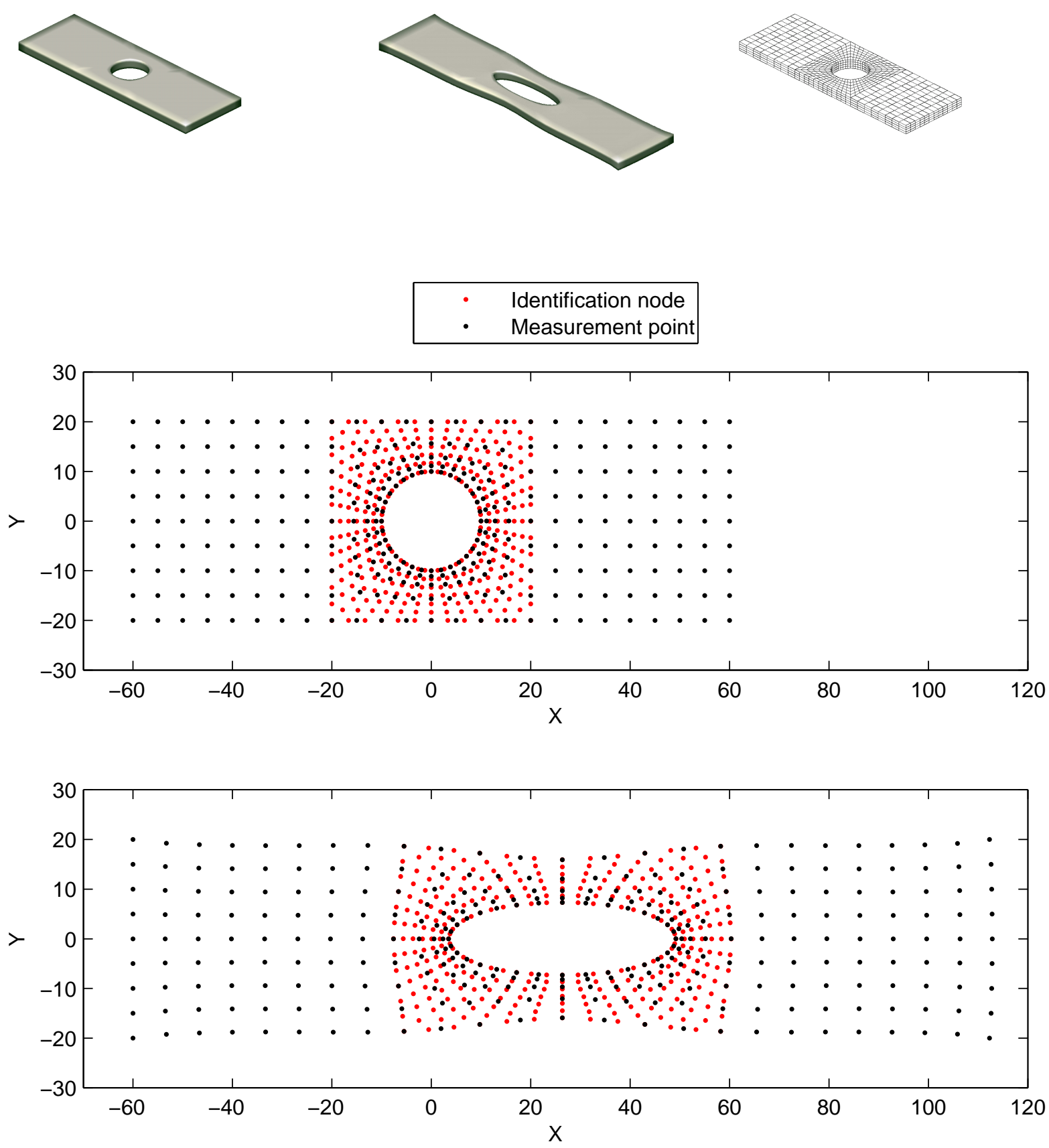

Figure 4.4: Example of a plate with a hole with two-dimensional measured displacements. Top: specimen in the reference configuration, specimen in the deformed configuration, and discretization of an associated FE model. Bottom: Interpolation of the displacements of the measurement points on the coordinates of the identification nodes. 

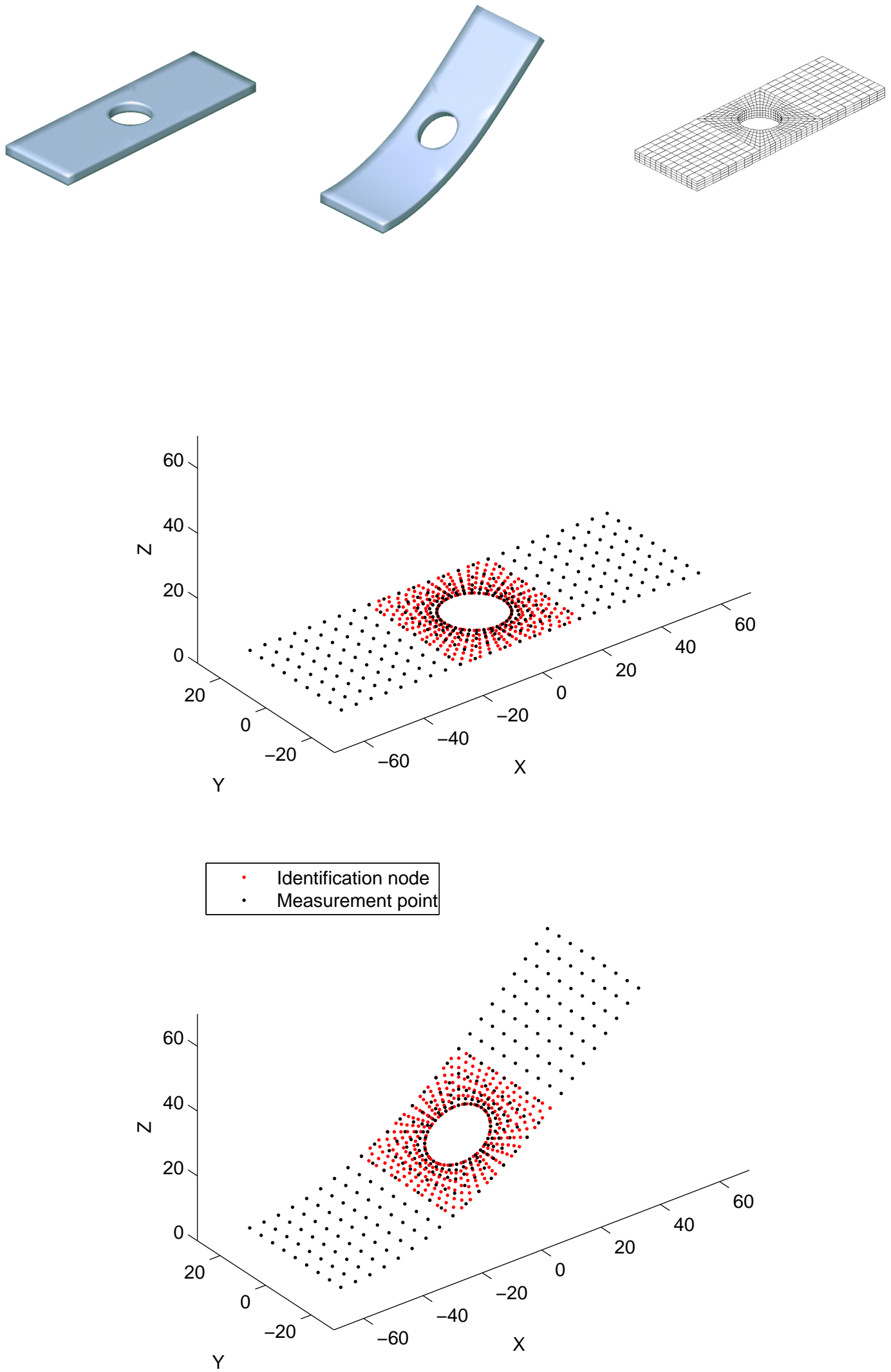

Figure 4.5: Example of a plate with a hole with three-dimensional measured displacements. Top: specimen in the reference configuration, specimen in the deformed configuration, and discretization of an associated FE model. Bottom: Interpolation of the displacements of the measurement points on the coordinates of the identification nodes. 


\subsection{Solution of the inverse problem}

The common procedure for the identification of material parameters is the minimization of a least squares problem in which the measured displacement fields and corresponding displacement fields of the FEM simulation are compared. The forces acting on the specimen in the experiment are the Neumann boundary conditions on the specimen in the FEM simulation. Before starting the identification process, the experimental results are made comparable to the FEM results which is done by an interpolation technique as described in the previous section. Then the objective function $f(\boldsymbol{\kappa})$ depending on the material parameters $\boldsymbol{\kappa}$ can be formulated as

$$
f(\boldsymbol{\kappa})=\frac{1}{2} \sum_{k=1}^{N}\left[\overline{\boldsymbol{u}}_{k}(\boldsymbol{\kappa})-\overline{\boldsymbol{u}}_{k}^{e x p}\right]^{2} .
$$

The domain on the specimen which is of interest for the identification leads to the choice of identification nodes. The corresponding displacements are assembled in the vectors $\overline{\boldsymbol{u}}_{k}(\boldsymbol{\kappa})$ and $\overline{\boldsymbol{u}}_{k}^{\text {exp }}$. The denomination $\overline{\boldsymbol{u}}_{k}(\boldsymbol{\kappa})$ denotes nodal displacements of the simulation, while $\overline{\boldsymbol{u}}_{k}^{\text {exp }}$ characterizes measured displacements after interpolation on the coordinates of the identification nodes. For simplicity, the index $k$ generally represents different timeor load steps and identification nodes.

The optimization algorithms for the minimization of the least squares functional can be classified in different ways (see, e.g. Mahnken [43]). One classification differs between algorithms which work by using only the computation of the objective function and algorithms which also necessitate the respective gradient (gradient methods). Gradient methods are referred to as Newton-like methods. Another classification is to distinguish between deterministic and stochastic methods. A deterministic algorithm will always produce the same result when given the same starting values, whereas stochastic methods are working by using random numbers for optimization. For an overview of these different optimization methods see, e.g. Mahnken [43], Bazaraa et al. [5], Bertsekas [7], Luenberger [38], Dennis and Schnabel [14], Nocedal and Wright [58], Spellucci [71] and Powell [61].

The objective function (4.8) is, in general, non-convex which means that in addition to the global minimum local minima might occur. As an advantage, stochastic methods have the ability to find the global minimum. A disadvantage is the high number of computations in comparison to deterministic methods. Since usually the computations for material models within the FE method are expensive even for the direct problem, the exclusive use of stochastic methods is not advisable. Deterministic gradient based methods, however, exhibit fast convergence but have the disadvantage of not overcoming (all) local minima. Therefore, a hybrid method - which has been applied and described in the work of, e.g. Mahnken and Stein [49] and Mahnken [43] - is used. Hereby, first the gradient descent method is applied to a number of randomly selected individual parameter sets. This combination allows to exploit the fast convergence of the Levenberg-Marquardt method to find different local minima. If the iteration process is trapped in a local minimum, the iteration is repeated with new starting points by changing individual parameters manually which is on the one hand a check for the robustness of the result. On the other hand 
this can also lead to another local minimum with a smaller value for the weighted sum of squared differences.

Using gradient based methods for the above minimization problem, the gradient of the objective function is calculated

$$
\partial \boldsymbol{\kappa} f(\boldsymbol{\kappa})=\sum_{k=1}^{N}\left[\overline{\boldsymbol{u}}_{k}(\boldsymbol{\kappa})-\overline{\boldsymbol{u}}_{k}^{e x p}\right] \cdot \partial_{\boldsymbol{\kappa}} \overline{\boldsymbol{u}}_{k}(\boldsymbol{\kappa}),
$$

which must vanish as a necessary condition for a local (or perhaps the global) minimum. The general iteration algorithm of gradient based optimization methods yields

$$
\boldsymbol{\kappa}^{(j+1)}=\boldsymbol{\kappa}^{(j)}-\alpha^{(j)} \boldsymbol{H}^{(j)} \cdot \partial \boldsymbol{\kappa} f\left(\boldsymbol{\kappa}^{(j)}\right)
$$

with the step length $\alpha^{(j)} \in[0,1]$ which we, nevertheless, assume as $\alpha^{(j)}=1$.

In subsection 4.4.1 first the Newton iteration matrix $\boldsymbol{H}=\boldsymbol{H}_{N}$ - generally giving quadratic convergence for optimization - is described and the Gauss-Newton iteration matrix $\boldsymbol{H}=\boldsymbol{H}_{G N}$ is deduced which can give quadratic convergence in special cases as well. In subsection 4.4.2 the efficient Levenberg-Marquardt iteration matrix $\boldsymbol{H}=\boldsymbol{H}_{L M}$ - a damped Gauss-Newton iteration matrix which is applied for the parameter identification in this work - is presented.

\subsubsection{The Gauss-Newton method}

The Hessian of $f(\boldsymbol{\kappa})$ yields

$$
\partial_{\boldsymbol{\kappa}}^{2} f(\boldsymbol{\kappa})=\sum_{k=1}^{N}\left[\overline{\boldsymbol{u}}_{k}(\boldsymbol{\kappa})-\overline{\boldsymbol{u}}_{k}^{e x p}\right] \cdot \partial_{\boldsymbol{\kappa}}^{2} \overline{\boldsymbol{u}}_{k}(\boldsymbol{\kappa})+\partial_{\boldsymbol{\kappa}} \overline{\boldsymbol{u}}_{k}(\boldsymbol{\kappa}) \otimes \partial_{\boldsymbol{\kappa}} \overline{\boldsymbol{u}}_{k}(\boldsymbol{\kappa}),
$$

and consequently the Newton iteration matrix is defined as $\left(\partial_{\boldsymbol{\kappa}}^{2} f(\boldsymbol{\kappa})\right)^{-1}=\boldsymbol{H}_{N}$. Therefore Newton's method for optimization is equivalent to iteratively minimizing a local quadratic approximation to the objective function. The first part of the Hessian contains information about the curvature of the objective function which are specified by the second derivatives $\partial_{\boldsymbol{\kappa}}^{2} \overline{\boldsymbol{u}}_{k}(\boldsymbol{\kappa})$. The second part of the Hessian is determined by the gradients $\partial_{\boldsymbol{\kappa}} \overline{\boldsymbol{u}}_{k}(\boldsymbol{\kappa})$. By assuming that near the solution the residua of the objective function yield small values, the first part of the Hessian can be neglected and this yields the Gauss-Newton iteration matrix

$$
\boldsymbol{H}_{G N}=\left[\sum_{k=1}^{N} \partial_{\boldsymbol{\kappa}} \overline{\boldsymbol{u}}_{k}(\boldsymbol{\kappa}) \otimes \partial_{\boldsymbol{\kappa}} \overline{\boldsymbol{u}}_{k}(\boldsymbol{\kappa})\right]^{-1} .
$$

With the Gauss-Newton method quadratic convergence can be expected near to the solution. In general at least linear convergence is achieved. Due to instabilities (see also subsection 4.5) the components of the gradient of the objective function become relatively small in the vicinity of a local or the global minimum. A flat least squares functional leads to an ill conditioned Gauss-Newton matrix. The condition number is defined as

$$
k=\frac{\lambda_{\min }}{\lambda_{\max }}
$$

whereby $\lambda_{i}, i=1, \ldots, n_{p}$ are the eigenvalues and $n_{p}$ is the number of material parameters. 


\subsubsection{The Levenberg-Marquardt method}

If the approximated Hessian $\boldsymbol{H}_{G N}^{-1}$ is ill-conditioned at the solution or not positive definite a damped Gauss-Newton method - the Levenberg-Marquardt method - can be applied. The iteration matrix yields

$$
\boldsymbol{H}_{L M}=\left[\left[\sum_{k=1}^{N} \partial_{\boldsymbol{\kappa}} \overline{\boldsymbol{u}}_{k}(\boldsymbol{\kappa}) \otimes \partial \boldsymbol{\kappa} \overline{\boldsymbol{u}}_{k}(\boldsymbol{\kappa})\right]+\mu \boldsymbol{I}\right]^{-1}
$$

wherein $\boldsymbol{I}$ denotes the identity matrix and $\mu$ is a damping factor which is chosen as small as possible with $\mu>0$ so that $\boldsymbol{H}_{L M}^{-1}$ is positive definite. Therefore - within the iteration algorithm as described in equation 4.10 - the step $\boldsymbol{h}_{L M}^{(j)}=\boldsymbol{\kappa}^{(j+1)}-\boldsymbol{\kappa}^{(j)}=\boldsymbol{H}_{L M}^{(j)} \cdot \partial_{\boldsymbol{\kappa}} f\left(\boldsymbol{\kappa}^{(j)}\right)$ is a descent direction.

The damping factor is chosen according to the iteration process (see e.g. Bazaraa et al. [5]), whereby $\mu$ are increasing if $\boldsymbol{H}_{G N}^{-1}$ is badly conditioned and if the iteration is far from a solution. By choosing larger values for $\mu$ the iteration step crosses over to a steepest descent direction which is given by $\boldsymbol{h}_{L M}^{(j)} \simeq-[1 / \mu] \partial_{\boldsymbol{\kappa}} f(\boldsymbol{\kappa})$. If the iteration process for $\boldsymbol{\kappa}$ becomes stable - near a local or the global minimum $-\mu$ can be decreased. Thereby, for very small $\mu$ the iteration matrix yields $\boldsymbol{H}_{L M}^{-1} \simeq \boldsymbol{H}_{G N}^{-1}$ and therefore quadratic convergence might be achieved.

\subsection{Stability investigations}

In contrast to the direct problem the nonlinear optimization problem (4.8) is, in general, an ill-posed problem. According to Hadamard, a mathematical problem is called wellposed if

- for all admissible data, a solution exists,

- for all admissible data, the solution is unique, and

- the solution depends continuously on the data.

If one of these properties is violated, the problem is called ill-posed. The non-convexity of the least squares functional and instabilities (the solution does not depend continuously on the data) in the solution might lead to the impracticality of finding an unique solution or even no solution (see, e.g. Banks and Kunisch [2], Baumeister [4], Bui [13], Louis [36] and Mahnken [43]). The reason for instabilities and therefore the difficulties in determining a reliable set of material parameters arise from different facts which can be summarized in three groups which are first the error of the simulation and secondly and thirdly the insufficiency and uncertainties of the experiments (see Mahnken [43]). The error of the simulation is due to the fact that a material model cannot perfectly describe all characteristics of a material. Moreover, within the solution of the direct problem usually different numerical approximations in time and space are made. In addition, the experiments for the parameter identification have to be coordinated with the characteristics of the constitutive law, so that all individual material parameters are activated within the 
identification process. Otherwise the experimental data is insufficient. The uncertainties within the experiments have two main reasons: Firstly stochastic noise in the measurement data is inevitable and depends on the chosen measurement system in combination with the dimension of the measured data. Secondly scattering of measurement data has to be taken into account. On the one hand inhomogeneities within the specimens can cause scattering. On the other hand serious scattering between different specimen due to the production process can appear. Note that usually scattering has a much bigger influence on the identification process and the identified parameters than it is the case for measurement noise.

Moreover, it needs to be mentioned that even for very small data errors the error in the solution can be large. In practical applications, exact measurement data are often not given and only a noisy scattered version is available.

One of the reasons for instabilities in least squares problems consists in possible overparametrization which can be caused since the experimental data provides too many parameters resulting in (almost) linear dependencies between the parameters (see Mahnken [40]). Moreover, instabilities can be caused since the experimental data provides too less information for all mechanical processes the model is able to describe.

A suitable indicator for the dependencies between the parameters among each other is the correlation matrix $K_{m n}$, the entries of which are calculated via the Gauss-Newton matrix by (see e.g. Ekh [15] and Kreißig et al. [31])

$$
\begin{aligned}
& \boldsymbol{T}=\boldsymbol{H}_{G N}^{-1}, \\
& K_{m n}=\frac{T_{m n}}{\sqrt{T_{m m} T_{n n}}},
\end{aligned}
$$

with $-1 \leq K_{m n} \leq 1$ and $K_{m m}=1$. The smaller the value of $\left|K_{m n}\right|$, the smaller is the correlation between the parameters $\kappa_{m}$ and $\kappa_{n}$. In contrast, if $\left|K_{m n}\right|$ approaches 1 , the dependency between the corresponding parameters increases. 


\section{Sensitivity Analysis}

The use of a gradient based iteration method for the solution of the least squares problem given in equation 4.8 necessitates the calculation of $\partial_{\boldsymbol{\kappa}} \overline{\boldsymbol{u}}_{k}\left(\boldsymbol{\kappa}^{(j)}\right)$ for each iteration step $(j)$ of the parameter identification algorithm.

Following the approach of Mahnken and Stein [48] for the analytical evaluation of sensitivities for inelastic models in the frame of the FE method in section 5.1, first the global solution of the direct problem is considered in subsection 5.1.1. In subsection 5.1.2 the computation of $\partial_{\boldsymbol{\kappa}} \overline{\boldsymbol{u}}_{k}\left(\boldsymbol{\kappa}{ }^{(j)}\right)$ within the global FE algorithm is shown. The necessary sensitivity analysis for the model of finite viscoelasticity on the constitutive level of the FE algorithm is presented in subsection 5.1.3.

The finite difference method for the model of finite viscoelasticity is considered in section 5.2 .

\subsection{Direct differentiation method}

\subsubsection{Global solution of the direct problem}

The static local spatial equilibrium equation yields

$$
\operatorname{div} \boldsymbol{\sigma}+\mathbf{b}=\mathbf{0}
$$

whereby $\boldsymbol{\sigma}$ are the Cauchy stresses and $\mathbf{b}$ are the body forces. The FE discretization yields the discretized equilibrium equations for individual element nodes $I=1,2, \ldots, n_{n d}$ to (see, e.g. Hughes [26], Wriggers [75], Reddy [62] and Zienkewicz and Taylor [76])

$$
\boldsymbol{r}_{I}=\boldsymbol{f}_{I}^{i n t}-\boldsymbol{f}_{I}^{e x t}=\mathbf{0}
$$

with the nodal residuum $\boldsymbol{r}_{I}$ and the internal and external nodal forces $\boldsymbol{f}_{I}^{\text {int }}$ and $\boldsymbol{f}_{I}^{\text {ext }}$. The internal nodal forces are computed by

$$
\boldsymbol{f}_{I}^{i n t}=\int_{\mathcal{B}_{t}} \partial_{\boldsymbol{x}}^{t} \boldsymbol{N}_{I} \cdot \boldsymbol{\sigma}(\boldsymbol{u}) d v
$$

whereby $\boldsymbol{u}$ are the displacements and $\boldsymbol{N}_{I}$ characterize the shape functions. Thus the nodal residuum yields

$$
\boldsymbol{r}_{I}(\boldsymbol{u})=\int_{\mathcal{B}_{t}} \partial_{\boldsymbol{x}}^{t} \boldsymbol{N}_{I} \cdot \boldsymbol{\sigma}(\boldsymbol{u}) d v-\boldsymbol{f}_{I}^{e x t} \quad \forall I=1,2, \ldots, n_{n d}
$$

For the computation of the displacements $\boldsymbol{u}$ which is done by the solution of the resulting global residuum by means of a Newton-Raphson method first the linearization of the element residuum is considered

$$
\sum_{I=1}^{n_{n d}} \frac{\partial \boldsymbol{r}_{I}\left(\boldsymbol{u}_{J}\right)}{\partial \boldsymbol{u}_{J}} \cdot \Delta \boldsymbol{u}_{J} \approx-\boldsymbol{r}_{I}
$$


with

$$
\frac{\partial \boldsymbol{r}_{I}\left(\boldsymbol{u}_{J}\right)}{\partial \boldsymbol{u}_{J}}=\mathbf{k}_{I J} \quad \forall I, J=1,2, \ldots, n_{n d}
$$

The Jacobian of the element residuum for dead loading is the element stiffness matrix $\mathbf{k}_{I J}$

$$
\mathbf{k}_{I J}=\mathbf{k}_{I J}^{g e o}+\mathbf{k}_{I J}^{m a t}
$$

with a geometric part

$$
\mathbf{k}_{I J}^{g e o}=\int_{\mathcal{B}_{t}} \partial_{\boldsymbol{x}}^{t} \boldsymbol{N}_{I} \cdot \boldsymbol{\sigma} \cdot \partial_{\boldsymbol{x}}^{t} \boldsymbol{N}_{J} d v \boldsymbol{I}
$$

and material part

$$
\mathbf{k}_{I J}^{m a t}=\int_{\mathcal{B}_{t}} \partial_{\boldsymbol{x}}^{t} \boldsymbol{N}_{I} \cdot \mathbf{c} \cdot \partial_{\boldsymbol{x}}^{t} \boldsymbol{N}_{J} d v
$$

Remark 5.1.1 The tangent operator $\mathbf{c}$ in $\mathcal{B}_{t}$ is computed by push-forward transformation of

$$
\mathbf{c}=J^{-1} \boldsymbol{\varphi}_{*} \mathbf{C}=J^{-1} \boldsymbol{\varphi}_{*}\left(2 \frac{\partial \boldsymbol{S}(\boldsymbol{C})}{\boldsymbol{C}}\right)
$$

with the second Piola-Kirchhoff stress tensor $\boldsymbol{S}$ and the right Cauchy-Green tensor $\boldsymbol{C}$.

The global variables are assembled in terms of the respective elemental ones by

$$
\mathbf{K}=\mathbf{A}_{e=1}^{n_{e l e m}} \mathbf{k}_{I J}, \quad \Delta \boldsymbol{u}=\mathbf{A}_{e=1}^{n_{\text {elem }}} \Delta \boldsymbol{u}_{J}, \quad \boldsymbol{R}=\mathbf{A}_{e=1}^{n_{\text {elem }}} \boldsymbol{r}_{I},
$$

with the global residual vector $\boldsymbol{R}$ and the global stiffness matrix $\mathbf{K}$ - boundary conditions already being accounted for. The system of equations for the global incremental displacements $\Delta \boldsymbol{u}$ yields

$$
\mathbf{K} \cdot \Delta \boldsymbol{u}=-\boldsymbol{R}=\mathbf{0} .
$$

The increment $\Delta \boldsymbol{u}$ is the solution of

$$
\Delta \boldsymbol{u}=-\left[\mathbf{K}^{(m)}\right]^{-1} \cdot \boldsymbol{R}^{(m)}
$$

and the displacement field is updated via

$$
\boldsymbol{u}^{(m+1)}=\boldsymbol{u}^{(m)}+\Delta \boldsymbol{u}
$$

in the global iteration algorithm. 


\subsubsection{Global solution of the inverse problem}

As mentioned above for the solution of the inverse problem by means of gradient based methods the derivative $\partial_{\kappa} \boldsymbol{u}(\boldsymbol{\kappa})$ has to be computed. Considering the total differential of the converged state of the global iteration

$$
\frac{d \boldsymbol{R}}{d \boldsymbol{\kappa}}=\frac{\partial \boldsymbol{R}}{\partial \boldsymbol{u}} \cdot \frac{\partial \boldsymbol{u}}{\partial \boldsymbol{\kappa}}+\frac{d^{p} \boldsymbol{R}}{d^{p} \boldsymbol{\kappa}}=\mathbf{0},
$$

with $\partial \boldsymbol{R} / \partial \boldsymbol{u}=\mathbf{K}$ one obtains

$$
\frac{\partial \boldsymbol{u}}{\partial \boldsymbol{\kappa}}=-\mathbf{K}^{-1} \cdot \frac{\partial^{p} \boldsymbol{R}}{\partial \boldsymbol{\kappa}},
$$

where in analogy to the work by Mahnken and Stein [48] the notation $\partial^{p}(\bullet(\boldsymbol{\kappa})) / \partial \boldsymbol{\kappa}$ emphasizes that this derivative excludes the implicit dependence of $\boldsymbol{\kappa}$ via the displacements $\boldsymbol{u}(\boldsymbol{\kappa})$. Thus, the above partial parameter derivative for the global residual results in

$$
\begin{aligned}
\frac{\partial^{p} \boldsymbol{R}}{\partial \boldsymbol{\kappa}}=\frac{\partial^{p} \boldsymbol{F}^{\text {int }}}{\partial^{p} \boldsymbol{\kappa}} & =\mathbf{A}_{e=1}^{n_{\text {elem }}} \int_{\mathcal{B}_{t}} \nabla_{\boldsymbol{x}}^{\mathrm{T}} \boldsymbol{N}_{I} \cdot \frac{\partial^{p}}{\partial \boldsymbol{\kappa}}(\boldsymbol{\sigma}(\boldsymbol{u}, \boldsymbol{\kappa}) d v) \\
& =\mathbf{A}_{e=1}^{n_{\text {elem }}} \int_{\mathcal{B}_{t}} \nabla_{\boldsymbol{x}}^{\mathrm{T}} \boldsymbol{N}_{I} \cdot \frac{\partial^{p} \boldsymbol{\tau}(\boldsymbol{\kappa})}{\partial \boldsymbol{\kappa}} \frac{1}{J} d v,
\end{aligned}
$$

whereby $\boldsymbol{\tau}=J \boldsymbol{\sigma}$ are the Kirchhoff stresses with $J=\operatorname{det} \boldsymbol{F}$ denoting the local volume ratio performing a volume change $d v=J d V$ between the initial and the spatial configuration. Together with equation (5.16) the partial parameter derivative for the global displacement vector yields

$$
\frac{\partial \boldsymbol{u}}{\partial \boldsymbol{\kappa}}=-\mathbf{K}^{-1} \cdot \mathbf{A}_{e=1}^{n_{\text {elem }}} \int_{\mathcal{B}_{t}} \nabla_{\boldsymbol{x}}^{\mathrm{T}} \boldsymbol{N}_{I} \cdot \frac{\partial^{p} \boldsymbol{\tau}(\boldsymbol{\kappa})}{\partial \boldsymbol{\kappa}} \frac{1}{J} d v
$$

which shows that it remains to compute the partial parameter derivative for the Kirchhoff stresses which is done at the constitutive level of the FE algorithm.

\subsubsection{Sensitivity analysis for the model of finite viscoelasticity}

Equivalent to equation (3.39), the partial parameter derivative for the Kirchhoff stresses takes the form

$$
\frac{\partial^{p} \boldsymbol{\tau}}{\partial \kappa_{1}}=\frac{\partial^{p} \boldsymbol{\tau}^{\mathrm{eq}}}{\partial \kappa_{1}}+\sum_{\mathrm{k}=1}^{\mathrm{N}} \frac{\partial^{p} \boldsymbol{\tau}^{\mathrm{k}}}{\partial \kappa_{1}} .
$$

Obviously, the equilibrium Kirchhoff stresses $\boldsymbol{\tau}^{\mathrm{eq}}=\boldsymbol{\tau}^{\mathrm{eq}}(\boldsymbol{u}, \boldsymbol{\kappa})$ do not directly depend on internal variables. The related partial parameter derivative consequently reads

$$
\begin{aligned}
& \frac{\partial^{p} \tau_{\mathrm{a}}^{\mathrm{eq}}}{\partial \kappa_{\mathrm{1}}}=\frac{\partial^{p}}{\partial \kappa_{1}}\left(\frac{\partial \Psi^{\mathrm{eq}}}{\partial \lambda_{\mathrm{a}}} \lambda_{\mathrm{a}}\right), \\
& \frac{\partial^{p} \boldsymbol{\tau}^{\mathrm{eq}}}{\partial \kappa_{1}}=\sum_{\mathrm{a}=1}^{3} \frac{\partial^{p} \tau_{\mathrm{a}}^{\mathrm{eq}}}{\partial \kappa_{1}} \boldsymbol{n}_{\mathrm{a}} \otimes \boldsymbol{n}_{\mathrm{a}} .
\end{aligned}
$$


For the evaluation of the partial parameter derivative for the non-equilibrium parts of the Kirchhoff stresses the history dependency has to be taken into account (compare Mahnken and Stein [49], [42]). Consequently $\partial\left(\left[\boldsymbol{C}_{\mathrm{v}}^{\mathrm{k}}\right]^{-1}\right)_{t=t_{\mathrm{n}-1}} / \partial \boldsymbol{\kappa}$ is needed for the computation of $\partial^{p} \boldsymbol{\tau}^{\mathrm{k}}(\boldsymbol{\kappa}) / \partial \boldsymbol{\kappa}$ where $\left(\left[\boldsymbol{C}_{\mathrm{v}}^{\mathrm{k}}\right]^{-1}\right)_{t=t_{\mathrm{n}-1}}$ is the inverse of the viscous right Cauchy-Green tensor and $n-1$ indicates the previous time step. Thus at each time step $n$ the parameter derivative $\partial\left(\left[\boldsymbol{C}_{\mathrm{v}}^{\mathrm{k}}\right]^{-1}\right)_{t=t_{\mathrm{n}}} / \partial \boldsymbol{\kappa}$ has to be computed and stored for the next time step.

In summary, for the computation of $\partial^{p} \boldsymbol{\tau}^{\mathrm{k}}(\boldsymbol{\kappa}) / \partial \kappa_{1}$ the following steps [A1]-[A6] must be performed at time step $\mathrm{n}$ for each relaxation module $\mathrm{k}$ (the index $\mathrm{n}$ for the actual time step is omitted):

[A1] Partial parameter derivative for the elastic left Cauchy-Green trial tensor

$$
\frac{\partial^{p}\left[\boldsymbol{b}_{\mathrm{e}}^{\mathrm{k}}\right]_{\mathrm{tr}}}{\partial \kappa_{\mathrm{1}}}=\boldsymbol{F} \cdot \frac{\partial\left[\left[\boldsymbol{C}_{\mathrm{v}}^{\mathrm{k}}\right]^{-1}\right]_{t=t_{\mathrm{n}-1}}}{\partial \kappa_{1}} \cdot \boldsymbol{F}^{\mathrm{T}}
$$

[A2] Partial parameter derivative for the tensor basis ${ }^{1}$

$$
\frac{\partial^{p}\left(\boldsymbol{n}_{\mathrm{a}} \otimes \boldsymbol{n}_{\mathrm{a}}\right)}{\partial \kappa_{1}}=\frac{\partial\left(\boldsymbol{n}_{\mathrm{a}} \otimes \boldsymbol{n}_{\mathrm{a}}\right)}{\partial\left[\boldsymbol{b}_{\mathrm{e}}^{\mathrm{k}}\right]_{\mathrm{tr}}}: \frac{\partial^{p}\left[\boldsymbol{b}_{\mathrm{e}}^{\mathrm{k}}\right]_{\mathrm{tr}}}{\partial \kappa_{1}}
$$

[A3] Partial parameter derivative for the principal logarithmic elastic trial stretches

$$
\frac{\partial^{p}\left[\varepsilon_{\mathrm{a}_{\mathrm{e}}}^{\mathrm{k}}\right]_{\mathrm{tr}}}{\partial \kappa_{\mathrm{l}}}=\frac{1}{2} \frac{1}{\left[\lambda_{\mathrm{a}_{\mathrm{e}}}^{\mathrm{k}}\right]_{\mathrm{tr}}^{2}}\left[\boldsymbol{n}_{\mathrm{a}} \otimes \boldsymbol{n}_{\mathrm{a}}\right]: \frac{\partial^{p}\left[\boldsymbol{b}_{\mathrm{e}}^{\mathrm{k}}\right]_{\mathrm{tr}}}{\partial \kappa_{1}}
$$

[A4] Derivatives for the calculation of the principal elastic stretches. The matrix $K_{\mathrm{ab}}^{\mathrm{k}}$ is computed for the converged state of the local Newton-iteration (see equation 3.24)

$$
\begin{aligned}
& \frac{\partial \mathrm{r}_{\mathrm{a}}^{\mathrm{k}}}{\partial \eta_{\mathrm{v}}^{\mathrm{k}}}=-\Delta t \frac{1}{9\left[\eta_{\mathrm{v}}^{\mathrm{k}}\right]^{2}} \boldsymbol{\tau}^{\mathrm{k}}: \boldsymbol{g} \\
& \frac{\partial \mathrm{r}_{\mathrm{a}}^{\mathrm{k}}}{\partial \eta_{\mathrm{D}}^{\mathrm{k}}}=-\Delta t \frac{1}{2\left[\eta_{\mathrm{D}}^{\mathrm{k}}\right]^{2}}\left[\tau_{\mathrm{a}}^{\mathrm{k}}\right]^{\mathrm{dev}} \\
& \frac{\partial \mathrm{r}_{\mathrm{a}}^{\mathrm{k}}}{\partial\left[\varepsilon_{\mathrm{b}_{\mathrm{e}}^{\mathrm{k}}}^{\mathrm{k}}\right]_{\mathrm{tr}}}=-\delta_{\mathrm{ab}} \\
& \frac{\partial \mathrm{r}_{\mathrm{a}}^{\mathrm{k}}}{\partial\left[\tau_{\mathrm{a}}^{\mathrm{k}}\right]^{\mathrm{dev}}}=\Delta t \frac{1}{2 \eta_{\mathrm{D}}^{\mathrm{k}}} \\
& \frac{\partial \mathrm{r}_{\mathrm{a}}^{\mathrm{k}}}{\partial\left(\boldsymbol{\tau}^{\mathrm{k}}: \boldsymbol{g}\right)}=\Delta t \frac{1}{9 \eta_{\mathrm{v}}^{\mathrm{k}}} \\
& \frac{\partial\left[\tau_{\mathrm{a}}^{\mathrm{k}}(\boldsymbol{\kappa})\right]^{\mathrm{dev}}}{\partial \kappa_{1}}=\sum_{\mathrm{b}=1}^{3} \frac{\partial}{\partial \overline{\mathrm{b}}_{\mathrm{b}_{\mathrm{e}}^{\mathrm{k}}}^{\mathrm{k}}}\left(\frac{\partial\left[\Psi^{\mathrm{k}}(\boldsymbol{\kappa})\right]^{\mathrm{dev}}}{\partial \kappa_{1}}\right) \frac{\partial \overline{\mathrm{b}}_{b_{\mathrm{e}}}^{\mathrm{k}}}{\partial \lambda_{\mathrm{a}_{\mathrm{e}}}^{\mathrm{k}}} \lambda_{\mathrm{a}_{\mathrm{e}}}^{\mathrm{k}} \\
& \frac{\partial\left(\boldsymbol{\tau}^{\mathrm{k}}(\boldsymbol{\kappa}): \boldsymbol{g}\right)}{\partial \kappa_{1}}=3 \frac{\partial}{\partial J_{\mathrm{e}}^{\mathrm{k}}}\left(\frac{\partial\left[\Psi^{\mathrm{k}}(\boldsymbol{\kappa})\right]^{\mathrm{vol}}}{\partial \kappa_{1}}\right) J_{\mathrm{e}}^{\mathrm{k}}
\end{aligned}
$$

\footnotetext{
${ }^{1}$ See Appendix A.1 for the calculation of $\partial\left(\boldsymbol{n}_{\mathrm{a}} \otimes \boldsymbol{n}_{\mathrm{a}}\right) / \partial\left[\boldsymbol{b}_{\mathrm{e}}^{\mathrm{k}}\right]_{\mathrm{tr}}$.
} 


$$
\begin{aligned}
\frac{\partial^{p} \varepsilon_{\mathrm{b}_{\mathrm{e}}}^{\mathrm{k}}}{\partial \kappa_{1}}= & -\left[K_{\mathrm{ab}}^{\mathrm{k}}\right]^{-1}\left[\frac{\partial \mathrm{r}_{\mathrm{a}}^{\mathrm{k}}}{\partial \eta_{\mathrm{v}}^{\mathrm{k}}} \frac{\partial \eta_{\mathrm{v}}^{\mathrm{k}}}{\partial \kappa_{1}}+\frac{\partial \mathrm{r}_{\mathrm{a}}^{\mathrm{k}}}{\partial \eta_{\mathrm{D}}^{\mathrm{k}}} \frac{\partial \eta_{\mathrm{D}}^{\mathrm{k}}}{\partial \kappa_{1}}+\frac{\partial \mathrm{r}_{\mathrm{a}}^{\mathrm{k}}}{\partial\left[\varepsilon_{\mathrm{b}_{\mathrm{e}}}^{\mathrm{k}}\right]_{\mathrm{tr}}} \frac{\partial^{p}\left[\varepsilon_{\mathrm{b}_{\mathrm{e}}}^{\mathrm{k}}\right]_{\mathrm{tr}}}{\partial \kappa_{1}}\right. \\
& \left.+\frac{\partial \mathrm{r}_{\mathrm{a}}^{\mathrm{k}}}{\partial\left[\tau_{\mathrm{a}}^{\mathrm{k}}\right]^{\operatorname{dev}}} \frac{\partial\left[\tau_{\mathrm{a}}^{\mathrm{k}}(\boldsymbol{\kappa})\right]^{\mathrm{dev}}}{\partial \kappa_{1}}+\frac{\partial \mathrm{r}_{\mathrm{a}}^{\mathrm{k}}}{\partial\left(\boldsymbol{\tau}^{\mathrm{k}}: \boldsymbol{g}\right)} \frac{\partial\left(\boldsymbol{\tau}^{\mathrm{k}}(\boldsymbol{\kappa}): \boldsymbol{g}\right)}{\partial \kappa_{1}}\right] \\
\frac{\partial^{p} \lambda_{\mathrm{a}_{\mathrm{e}}}^{\mathrm{k}}=}{\partial \kappa_{1}}= & \lambda_{\mathrm{a}_{\mathrm{e}}}^{\mathrm{k}} \frac{\partial^{p} \varepsilon_{\mathrm{a}_{\mathrm{e}}}^{\mathrm{k}}}{\partial \kappa_{1}}
\end{aligned}
$$

[A5] Partial parameter derivative for the principal Kirchhoff stresses

$$
\begin{aligned}
& \frac{\partial \tau_{\mathrm{a}}^{\mathrm{k}}(\boldsymbol{\kappa})}{\partial \kappa_{1}}=\frac{\partial\left[\tau_{\mathrm{a}}^{\mathrm{k}}(\boldsymbol{\kappa})\right]^{\operatorname{dev}}}{\partial \kappa_{1}}+\frac{1}{3} \frac{\partial\left(\boldsymbol{\tau}^{\mathrm{k}}(\boldsymbol{\kappa}): \boldsymbol{g}\right)}{\partial \kappa_{1}} \\
& \frac{\partial^{p} \tau_{\mathrm{a}}^{\mathrm{k}}\left(\boldsymbol{\kappa}, \lambda_{\mathrm{b}_{\mathrm{e}}}^{\mathrm{k}}\right)}{\partial \kappa_{1}}=\frac{\partial \tau_{\mathrm{a}}^{\mathrm{k}}(\boldsymbol{\kappa})}{\partial \kappa_{1}}+\frac{\partial \tau_{\mathrm{a}}^{\mathrm{k}}\left(\lambda_{\mathrm{b}_{\mathrm{e}}}^{\mathrm{k}}\right)}{\partial \lambda_{\mathrm{b}_{\mathrm{e}}}^{\mathrm{k}}} \frac{\partial^{p} \lambda_{\mathrm{b}_{\mathrm{e}}}^{\mathrm{k}}}{\partial \kappa_{1}}
\end{aligned}
$$

[A6] Partial parameter derivative for the Kirchhoff stresses

$$
\frac{\partial^{p} \boldsymbol{\tau}^{\mathrm{k}}}{\partial \kappa_{1}}=\sum_{\mathrm{a}=1}^{3}\left[\frac{\partial^{p} \tau_{\mathrm{a}}^{\mathrm{k}}}{\partial \kappa_{1}} \boldsymbol{n}_{\mathrm{a}} \otimes \boldsymbol{n}_{\mathrm{a}}+\tau_{\mathrm{a}_{\mathrm{e}}}^{\mathrm{k}} \frac{\partial^{p}\left(\boldsymbol{n}_{\mathrm{a}} \otimes \boldsymbol{n}_{\mathrm{a}}\right)}{\partial \kappa_{1}}\right]
$$

Furthermore, the computation of $\partial\left[\left[\boldsymbol{C}_{\mathrm{v}}^{\mathrm{k}}\right]^{-1}\right]_{t=t_{\mathrm{n}}} / \partial \kappa_{1}$ is based on the following steps [B1][B6] referred to time step $\mathrm{n}$ for each relaxation module $\mathrm{k}$ (the index $\mathrm{n}$ for the actual time step is omitted):

[B1] Parameter derivative for the elastic left Cauchy-Green trial tensor

$$
\frac{\partial\left[\boldsymbol{b}_{\mathrm{e}}^{\mathrm{k}}\right]_{\mathrm{tr}}}{\partial \kappa_{\mathrm{1}}}=\boldsymbol{F} \cdot \frac{\partial\left[\left[\boldsymbol{C}_{\mathrm{v}}^{\mathrm{k}}\right]^{-1}\right]_{t=t_{\mathrm{n}-1}}}{\partial \kappa_{1}} \cdot \boldsymbol{F}^{\mathrm{T}}+2 \operatorname{sym}\left(\frac{\partial \boldsymbol{F}}{\partial \kappa_{1}} \cdot \boldsymbol{F}^{-1} \cdot\left[\boldsymbol{b}_{\mathrm{e}}^{\mathrm{k}}\right]_{\mathrm{tr}}\right)
$$

[B2] Parameter derivative for the tensor basis ${ }^{2}$

$$
\frac{\partial\left(\boldsymbol{n}_{\mathrm{a}} \otimes \boldsymbol{n}_{\mathrm{a}}\right)}{\partial \kappa_{1}}=\frac{\partial\left(\boldsymbol{n}_{\mathrm{a}} \otimes \boldsymbol{n}_{\mathrm{a}}\right)}{\partial\left[\boldsymbol{b}_{\mathrm{e}}^{\mathrm{k}}\right]_{\mathrm{tr}}}: \frac{\partial\left[\boldsymbol{b}_{\mathrm{e}}^{\mathrm{k}}\right]_{\mathrm{tr}}}{\partial \kappa_{1}}
$$

[B3] Parameter derivative for the principal logarithmic elastic trial stretches

$$
\frac{\partial\left[\varepsilon_{\mathrm{a}_{\mathrm{e}}}^{\mathrm{k}}\right]_{\mathrm{tr}}}{\partial \kappa_{1}}=\frac{1}{2} \frac{1}{\left[\lambda_{\mathrm{a}_{\mathrm{e}}}^{\mathrm{k}}\right]_{\mathrm{tr}}^{2}}\left[\boldsymbol{n}_{\mathrm{a}} \otimes \boldsymbol{n}_{\mathrm{a}}\right]: \frac{\partial\left[\boldsymbol{b}_{\mathrm{e}}^{\mathrm{k}}\right]_{\mathrm{tr}}}{\partial \kappa_{1}}
$$

[B4] Parameter derivative for the principal elastic stretches [see [A4]]

$$
\begin{aligned}
\frac{\partial \varepsilon_{\mathrm{b}_{\mathrm{e}}}^{\mathrm{k}}}{\partial \kappa_{\mathrm{l}}}= & -\left[K_{\mathrm{ab}}^{\mathrm{k}}\right]^{-1}\left[\frac{\partial \mathrm{r}_{\mathrm{a}}^{\mathrm{k}}}{\partial \eta_{\mathrm{v}}^{\mathrm{k}}} \frac{\partial \eta_{\mathrm{v}}^{\mathrm{k}}}{\partial \kappa_{1}}+\frac{\partial \mathrm{r}_{\mathrm{a}}^{\mathrm{k}}}{\partial \eta_{\mathrm{D}}^{\mathrm{k}}} \frac{\partial \eta_{\mathrm{D}}^{\mathrm{k}}}{\partial \kappa_{1}}+\frac{\partial \mathrm{r}_{\mathrm{a}}^{\mathrm{k}}}{\partial\left[\varepsilon_{\mathrm{b}_{\mathrm{e}}}^{\mathrm{k}}\right]_{\mathrm{tr}}} \frac{\partial\left[\varepsilon_{\mathrm{b}_{\mathrm{e}}}^{\mathrm{k}}\right]_{\mathrm{tr}}}{\partial \kappa_{1}}\right. \\
& \left.+\frac{\partial \mathrm{r}_{\mathrm{a}}^{\mathrm{k}}}{\partial\left[\tau_{\mathrm{a}}^{\mathrm{k}}\right]^{\operatorname{dev}}} \frac{\partial\left[\tau_{\mathrm{a}}^{\mathrm{k}}(\boldsymbol{\kappa})\right]^{\operatorname{dev}}}{\partial \kappa_{1}}+\frac{\partial \mathrm{r}_{\mathrm{a}}^{\mathrm{k}}}{\partial\left(\boldsymbol{\tau}^{\mathrm{k}}: \boldsymbol{g}\right)} \frac{\partial\left(\boldsymbol{\tau}^{\mathrm{k}}(\boldsymbol{\kappa}): \boldsymbol{g}\right)}{\partial \kappa_{1}}\right]
\end{aligned}
$$

\footnotetext{
${ }^{2}$ See Appendix A.1 for the calculation of $\partial\left(\boldsymbol{n}_{\mathrm{a}} \otimes \boldsymbol{n}_{\mathrm{a}}\right) / \partial\left[\boldsymbol{b}_{\mathrm{e}}^{\mathrm{k}}\right]_{\mathrm{tr}}$.
} 
[B5] Parameter derivative for the elastic eigenstretches

$$
\frac{\partial\left[\lambda_{\mathrm{a}_{\mathrm{e}}}^{\mathrm{k}}\right]^{2}}{\partial \kappa_{1}}=2\left[\lambda_{\mathrm{a}_{\mathrm{e}}}^{\mathrm{k}}\right]^{2} \frac{\partial \varepsilon_{\mathrm{a}_{\mathrm{e}}}^{\mathrm{k}}}{\partial \kappa_{1}}
$$

[B6] Parameter derivative for the elastic left Cauchy-Green tensor

$$
\frac{\partial \boldsymbol{b}_{\mathrm{e}}^{\mathrm{k}}}{\partial \kappa_{1}}=\sum_{\mathrm{a}=1}^{3}\left[\frac{\partial\left[\lambda_{\mathrm{a}_{\mathrm{e}}}^{\mathrm{k}}\right]^{2}}{\partial \kappa_{1}} \boldsymbol{n}_{\mathrm{a}} \otimes \boldsymbol{n}_{\mathrm{a}}+\left[\lambda_{\mathrm{a}_{\mathrm{e}}}^{\mathrm{k}}\right]^{2} \frac{\partial\left(\boldsymbol{n}_{\mathrm{a}} \otimes \boldsymbol{n}_{\mathrm{a}}\right)}{\partial \kappa_{1}}\right]
$$

[B7] Parameter derivative for the inverse of the viscous right Cauchy-Green tensor

$$
\frac{\partial\left[\left[\boldsymbol{C}_{\mathrm{v}}^{\mathrm{k}}\right]^{-1}\right]_{t=t_{\mathrm{n}}}}{\partial \kappa_{1}}=-2 \operatorname{sym}\left(\boldsymbol{F}^{-1} \cdot \frac{\partial \boldsymbol{F}}{\partial \kappa_{1}} \cdot\left[\left[\boldsymbol{C}_{\mathrm{v}}^{\mathrm{k}}\right]^{-1}\right]_{t=t_{\mathrm{n}-1}}\right)+\boldsymbol{F}^{-1} \cdot \frac{\partial \boldsymbol{b}_{\mathrm{e}}^{\mathrm{k}}}{\partial \kappa_{1}} \cdot \boldsymbol{F}^{-T}
$$

\section{Optimization scheme}

The optimization scheme for the solution of the inverse problem applying the direct differentiation method is illustrated in figure 5.1 (see also Mahnken and Stein [49]).

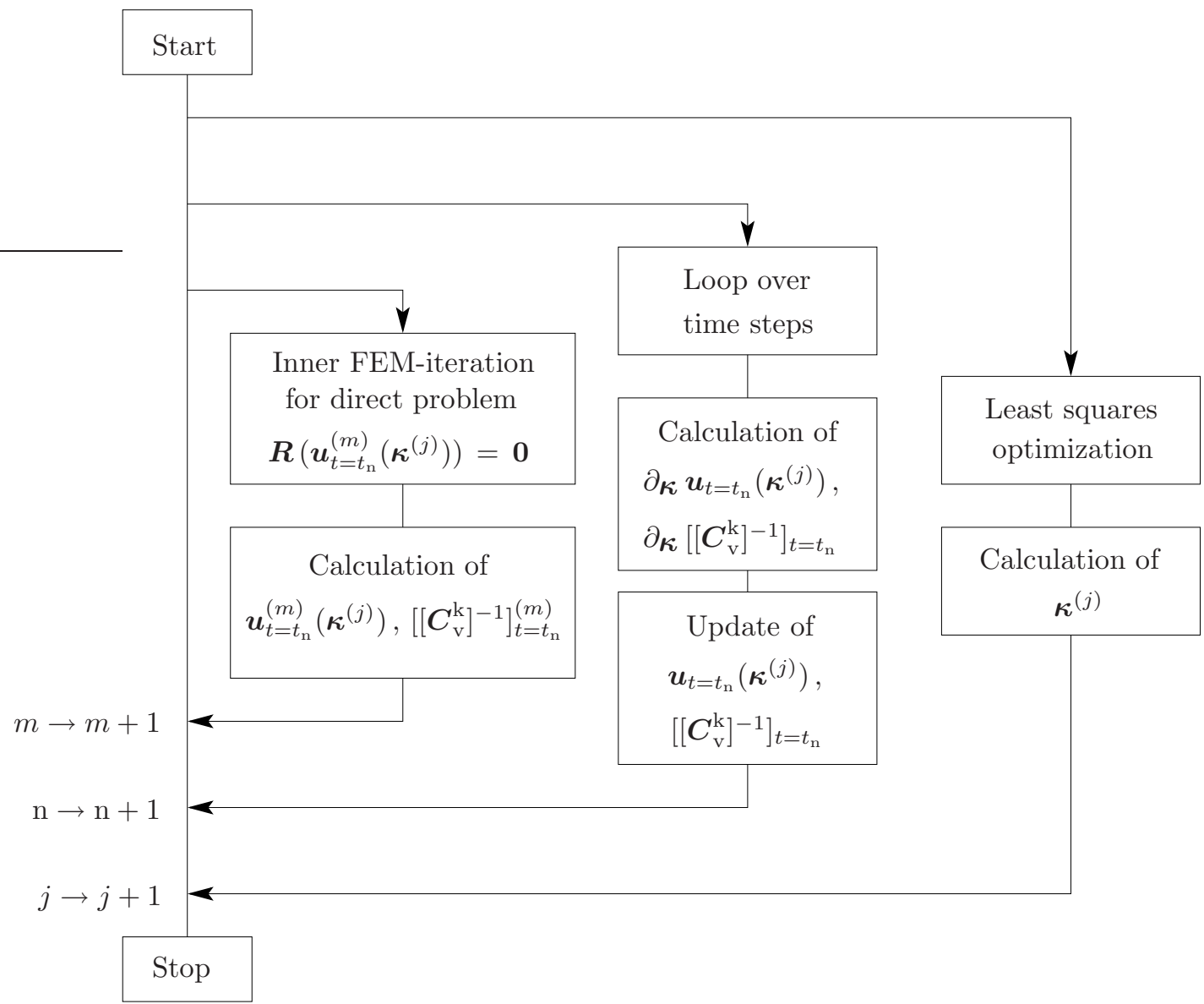

Figure 5.1: Optimization scheme 


\subsection{Finite difference method}

The numerical calculation of the parameter sensitivities $\partial_{\boldsymbol{\kappa}} \overline{\boldsymbol{u}}_{i j}(\boldsymbol{\kappa})$ yields

$$
\partial_{\kappa_{m}} \overline{\boldsymbol{u}}_{i j}(\boldsymbol{\kappa}) \approx \frac{\overline{\boldsymbol{u}}_{i j}\left(\boldsymbol{\kappa}+\epsilon \boldsymbol{e}_{m}\right)-\overline{\boldsymbol{u}}_{i j}(\boldsymbol{\kappa})}{\epsilon}
$$

with $m=1,2, \ldots, n_{M P}$ for the number of parameters of the applied viscoelastic material law. Here $\boldsymbol{e}_{m}$ is an unit vector with the entry 1 at position $m$ and the entries 0 for the other positions. The numerical pertubation is computed with sufficiently small $\epsilon$. 


\section{Parameter identification on the basis of virtual measurement data}

The consititutive routine for finite viscoelasticity - which dates back to the work by Reese and Govindjee [65] and has been reviewed in chapter 3 - is applied for the parameter identification for elastomeric materials in this work (see chapter 7). Since real measurement data is always subject to uncertainties which complicate the solution of the inverse problem (see section 4.5), it is advisable to conduct numerical studies for the respective material model first. Thereby, the possibility of instabilities within the identification iteration - arising from the structure of the viscoelastic model itself - can be assessed and therefore overparametrization might be avoided in the following identifications for real materials.

The constitutive routine for finite viscoelasticity bases on the hyperelastic Ogden material law as shown in subsection 2.3.2. In the following two sections the aim is to investigate, if the necessity of programming the Ogden material model with multiple terms $N_{T}>1$ for the isochoric part (see equation (2.32)) is given or if a simulation can also achieve good agreement to (virtual) measurement data by only programming the Ogden model with one term for the isochoric part. If a reduction of the material parameters does not lead to a deterioration of the quality of the respective simulation, it automatically helps to avoid instability problems due to overparametrization. Moreover, by application of a model structure with less material parameters, the computational costs for both, the direct and the inverse problem, can be reduced significantly.

In section 6.1 a parameter identification for hyperelasticity is conducted whereby for the simulation of the virtual measurement data an Ogden material with three isochoric terms is used and the identification is made for an Ogden material with only one respective term. Consequently, in subsection 6.2 for the viscoelastic model with one relaxation term the virtual measurement data is simulated with three terms for the equilibrium Ogden model and three terms for the Ogden model within the non-equilibrium part. The identification is conducted with only one term for each of the respective Ogden models.

Remark 6.0.1 It needs to be mentioned that with these two studies, if at all, only a tendency can be specified. The comparison of different structures of Ogden terms in the respective re-identification problem is made only for the chosen deformation range, FE model geometry and material parameters for the simulation of virtual measurement data.

Remark 6.0.2 Note that for all respective identification procedures the same FE discretization is used as for the respective simulation of the virtual measurement data. Therefore no interpolation of the (virtual) measurement data is necessary. 


\subsection{Finite Ogden hyperelasticity}

The virtual measurement data are the displacements $\overline{\boldsymbol{u}}_{i j}^{\exp }=\overline{\boldsymbol{u}}_{i j}^{\text {exp }}(\widetilde{\boldsymbol{\kappa}})$ which are simulated with the Ogden material with three terms $\left(N_{T}=3\right.$, see equation (2.32)). Thereby, the set of material parameters is given by $\widetilde{\boldsymbol{\kappa}}=\left[\widetilde{\mu}_{1}, \widetilde{\mu}_{2}, \widetilde{\mu}_{3}, \widetilde{\alpha}_{1}, \widetilde{\alpha}_{2}, \widetilde{\alpha}_{3}, \widetilde{\kappa}_{0}\right]^{\mathrm{T}}$ with the parameter values shown in table (6.1).

Table 6.1: Material parameter set $\widetilde{\kappa}$ for the simulation of virtual measurement data with Ogden hyperelasticity

\begin{tabular}{|l|l|l|l|l|l|l|}
\hline$\widetilde{\mu}_{1}$ & $\widetilde{\mu}_{2}$ & $\widetilde{\mu}_{3}$ & $\widetilde{\alpha}_{1}$ & $\widetilde{\alpha}_{2}$ & $\widetilde{\alpha}_{3}$ & $\widetilde{\kappa}_{0}$ \\
\hline \hline 9.0 & -9.0 & 15.0 & 2.8 & 2.2 & 1.2 & 350.0 \\
\hline$[\mathrm{MPa}]$ & {$[\mathrm{MPa}]$} & {$[\mathrm{MPa}]$} & {$[-]$} & {$[-]$} & {$[-]$} & {$[\mathrm{MPa}]$} \\
\hline
\end{tabular}

The simulation within the identification is carried out for an Ogden model with one term $\left(N_{T}=1\right)$ with the parameter set $\boldsymbol{\kappa}=\left[\mu_{1}, \alpha_{1}, k\right]^{\mathrm{T}}$.

For the simulations of the virtual three-dimensional measurement data and for the simulations within the identification routine the FE model shown in figure (6.1) is used and both simulations are identically force controlled with ten load steps with equal force load increments for each step.

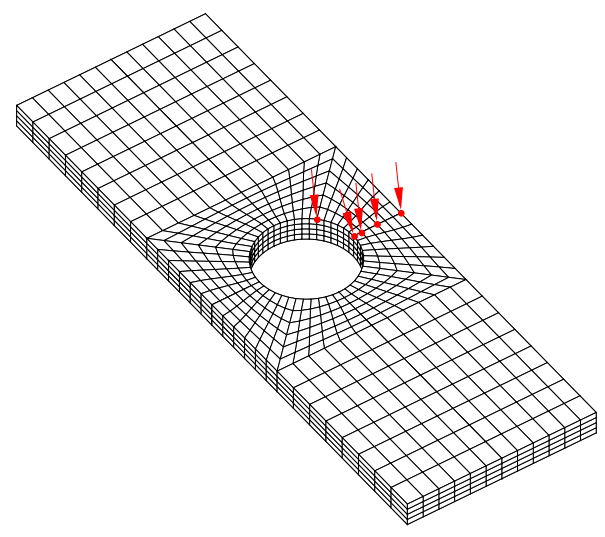

Figure 6.1: FE model with marked identification nodes.

The objective function for the inverse problem yields

$$
f(\boldsymbol{\kappa})=\frac{1}{2} \sum_{i=1}^{T} \sum_{j=1}^{N_{g}}\left[\overline{\boldsymbol{u}}_{i j}(\boldsymbol{\kappa})-\overline{\boldsymbol{u}}_{i j}^{\exp }\right]^{2}
$$

and is minimized by means of the Levenberg-Marquardt method. $T$ is the number of load steps with $T=10$ and $N_{g}$ is the number of identification nodes. The five $\left(N_{g}=5\right)$ identification nodes are marked in figure (6.1). The three-dimensional displacements $\overline{\boldsymbol{u}}_{i j}(\boldsymbol{\kappa})$ are simulated by the Ogden material for which $N_{T}=1$ holds. In tabular (6.2) and figure (6.5) the iteration is shown in which after eight iteration steps a local or maybe global minimum is determined. Since the solution is stable it might be assumed that the global minimum has been found. 
Table 6.2: Iteration for the identification for finite Ogden hyperelasticity.

\begin{tabular}{|r||l|l|l|l|}
\hline$n$ & $\mu_{1}$ & $\alpha_{1}$ & $\kappa_{0}$ & $f(\boldsymbol{\kappa})$ \\
\hline 0 & 15 & 5 & 1000 & 59.3111 \\
\hline 1 & 43.6343 & 1.3 & 10 & 1398.71 \\
\hline 2 & 60.4819 & 1.3 & 19.6197 & 52.7586 \\
\hline 3 & 27.8854 & 1.74212 & 42.2743 & 150.304 \\
\hline 4 & 39.4168 & 1.46877 & 72.2105 & 30.0739 \\
\hline 5 & 43.6623 & 1.44323 & 125.343 & 3.20162 \\
\hline 6 & 38.1302 & 1.61177 & 203.391 & 0.766273 \\
\hline 7 & 37.4445 & 1.65646 & 275.806 & 0.0553081 \\
\hline 8 & 37.179 & 1.6674 & 307.166 & 0.0325014 \\
\hline 9 & 37.1682 & 1.66802 & 309.662 & 0.0323618 \\
\hline 10 & 37.1682 & 1.66804 & 309.599 & 0.0323618 \\
\hline 11 & 37.1682 & 1.66804 & 309.601 & 0.0323618 \\
\hline & {$[\mathrm{MPa}]$} & {$[-]$} & {$[\mathrm{MPa}]$} & {$\left[\mathrm{mm}^{2}\right]$} \\
\hline
\end{tabular}

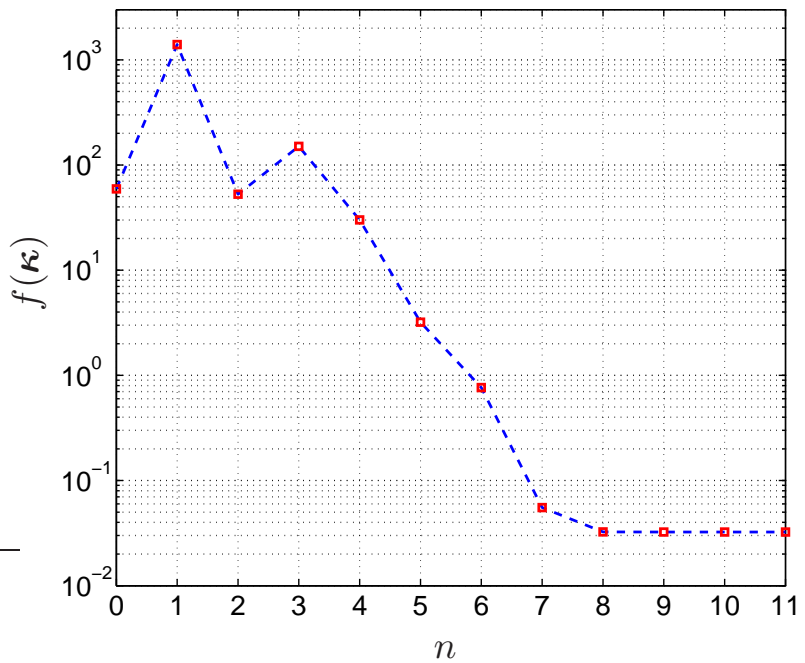

Figure 6.2: Sum of squared differences versus iteration steps for the identification of Ogden hyperelasticity.

Figure (6.3) shows that the deformed FE models for the virtual measurement data and for the solution of the identification problem optically (almost) coincide. Moreover, as presented in figure (6.4) for the tenth load step both FE simulations have almost the same distribution of stresses (here: euclidean norm of the deviatoric Cauchy stresses). The results of the identification show that an extension of the Ogden model to multiple terms within the sum for the deviatoric part of the energy might not be necessary for the later parameter identification for real materials. For the deformation range considered in this example the Ogden model with only three parameters can almost describe the same deformation behavior. Therefore it also follows that more terms with more parameters might lead to overparametrization and hence lead to instabilities very easily. Moreover, with a higher number of parameters the computational costs are increasing. 


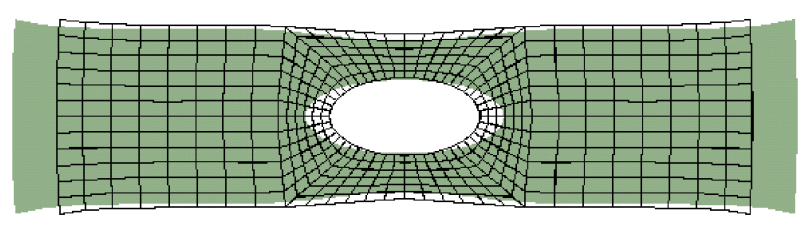

(a) Start of the identification iteration $(n=0)$.

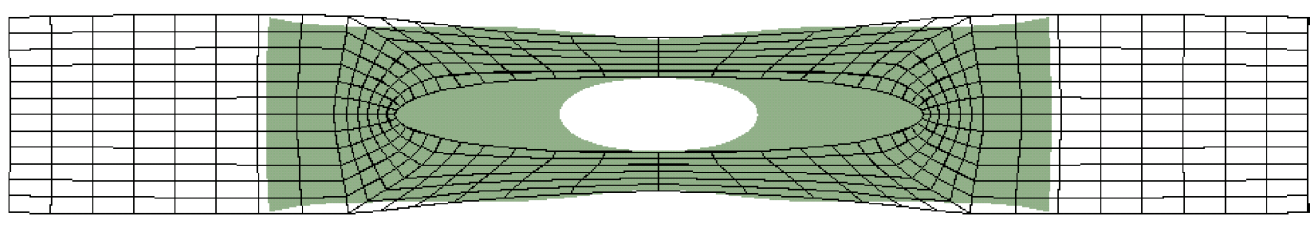

(b) After two iteration steps.

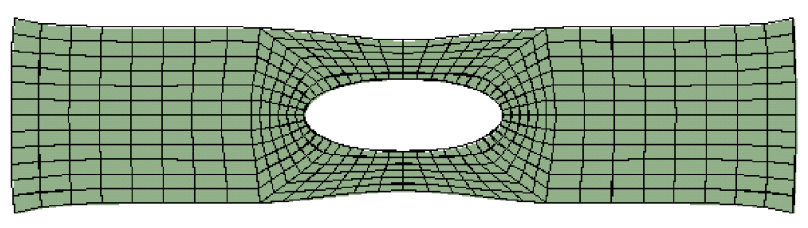

(c) End of the identification iteration $(n=11)$.

Figure 6.3: FE model of the Ogden material with three parameters over the virtual specimen during the iteration at the tenth load step.
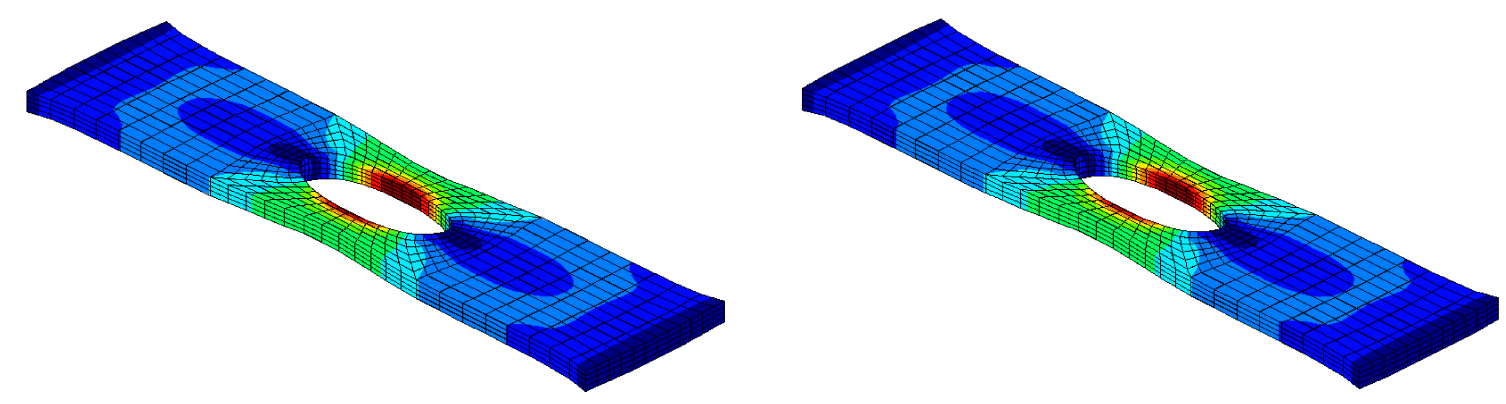

(a) FE simulation for the virtual measurement (b) FE simulation for the identified optimal matedata. rial parameters.

Figure 6.4: Comparison of the euclidean norm of the deviatoric Cauchy stresses at the tenth load step. 


\subsection{Finite viscoelasticity with one relaxation term}

The virtual measurement data are the displacements $\overline{\boldsymbol{u}}_{i j}^{\text {exp }}=\overline{\boldsymbol{u}}_{i j}^{\text {exp }}(\widetilde{\boldsymbol{\kappa}})$ which are simulated with the viscoelastic material with one relaxation term $(N=1$ in equation (3.8)). Thereby, within the equilibrium term of the free energy function $\Psi^{\mathrm{eq}}$ - as defined in equation $(3.60)$ - the part $\left[\Psi^{\mathrm{eq}}\right]^{\mathrm{dev}}$ consists of three terms, so that $\left(N_{T}=3\right)$ holds. For the one non-equilibrium part $\Psi^{k=1}$ the number of terms in $\left[\Psi^{\mathrm{k}=1}\right]^{\mathrm{dev}}$ is given by $\mathrm{N}_{\mathrm{T}}^{k=1}=3$ as well. Hence, the set of material parameters for the identification problem is $\widetilde{\kappa}=\left[\left[\widetilde{\mu}_{\text {eq }}\right]_{1},\left[\widetilde{\mu}_{\text {eq }}\right]_{2},\left[\widetilde{\mu}_{\text {eq }}\right]_{3},\left[\widetilde{\alpha}_{\text {eq }}\right]_{1},\left[\widetilde{\alpha}_{\text {eq }}\right]_{2},\left[\widetilde{\alpha}_{\text {eq }}\right]_{3}, \widetilde{\kappa}_{\text {eq }},\left[\widetilde{\mu}_{\text {neq }}^{\mathrm{k}=1}\right]_{1},\left[\widetilde{\mu}_{\text {neq }}^{\mathrm{k}=1}\right]_{2},\left[\widetilde{\mu}_{\text {neq }}^{\mathrm{k}=1}\right]_{3},\left[\widetilde{\alpha}_{\text {neq }}^{\mathrm{k}=1}\right]_{1},\left[\widetilde{\alpha}_{\text {neq }}^{\mathrm{k}=1}\right]_{2},\left[\widetilde{\alpha}_{\text {neq }}^{\mathrm{k}=1}\right]_{3}, \widetilde{\kappa}_{\text {neq }}^{\mathrm{k}=1}, \widetilde{\tau}^{\mathrm{k}=1}\right]^{\mathrm{T}}$ with the parameter values shown in table (6.3).

Table 6.3: Material parameter set $\widetilde{\kappa}$ for the simulation of virtual measurement data with finite viscoelasticity with one relaxation term.

\begin{tabular}{|c|c|c|c|c|c|c|c|}
\hline$\left[\widetilde{\mu}_{\mathrm{eq}}\right]_{1}$ & {$\left[\widetilde{\mu}_{\mathrm{eq}}\right]_{2}$} & {$\left[\widetilde{\mu}_{\mathrm{eq}}\right]_{3}$} & {$\left[\widetilde{\alpha}_{\text {eq }}\right]_{1}$} & {$\left[\widetilde{\alpha}_{\mathrm{eq}}\right]_{2}$} & {$\left[\widetilde{\alpha}_{\mathrm{eq}}\right]_{3}$} & $\widetilde{\kappa}_{\text {eq }}$ & \\
\hline 5.0 & -4.0 & $\begin{array}{l}1.0 \\
\end{array}$ & $\begin{array}{l}1.8 \\
\end{array}$ & $\begin{array}{l}-2.0 \\
\end{array}$ & 7.0 & 100.0 & \\
\hline$[\mathrm{MPa}]$ & {$[\mathrm{MPa}]$} & {$[\mathrm{MPa}]$} & {$[-]$} & {$[-]$} & {$[-]$} & {$[\mathrm{MPa}]$} & \\
\hline$\left[\widetilde{\mu}_{\text {neq }}^{\mathrm{k}=1}\right]_{1}$ & {$\left[\widetilde{\mu}_{\mathrm{neq}}^{\mathrm{k}=1}\right]_{2}$} & {$\left[\widetilde{\mu}_{\mathrm{neq}}^{\mathrm{k}=1}\right]_{3}$} & {$\left[\widetilde{\alpha}_{\mathrm{neq}}^{\mathrm{k}=1}\right]_{1}$} & {$\left[\widetilde{\alpha}_{\mathrm{neq}}^{\mathrm{k}=1}\right]_{2}$} & {$\left[\widetilde{\alpha}_{\mathrm{neq}}^{\mathrm{k}=1}\right]_{3}$} & $\begin{array}{c}\widetilde{\kappa}_{\text {neq }}^{\mathrm{k}=1} \\
\end{array}$ & $\widetilde{\tau}^{\mathrm{k}=1}$ \\
\hline 10.0 & -12.0 & 2.0 & 5.0 & $\begin{array}{l}-2.0 \\
\end{array}$ & 7 & 1.1 & 7.5 \\
\hline$[\mathrm{MPa}]$ & {$[\mathrm{MPa}]$} & {$[\mathrm{MPa}]$} & {$[-]$} & {$[-]$} & {$[-]$} & {$[\mathrm{MPa}]$} & {$[\mathrm{S}]$} \\
\hline
\end{tabular}

For the simulations of the virtual three-dimensional measurement data and for the simulations within the identification routine the FE model shown in figure (6.5) is applied. The simulated measurement data describes a creep test. Both simulations are identically force controlled with ten time steps with a time step length of $\Delta t=10 \mathrm{~s}$ as shown in figure (6.6).

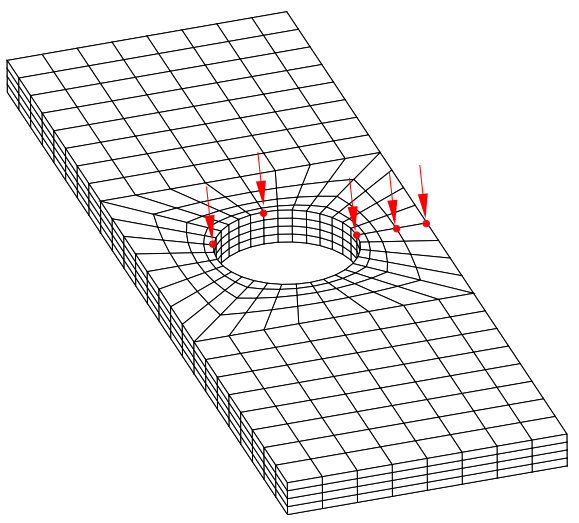

Figure 6.5: FE model with marked identification nodes.

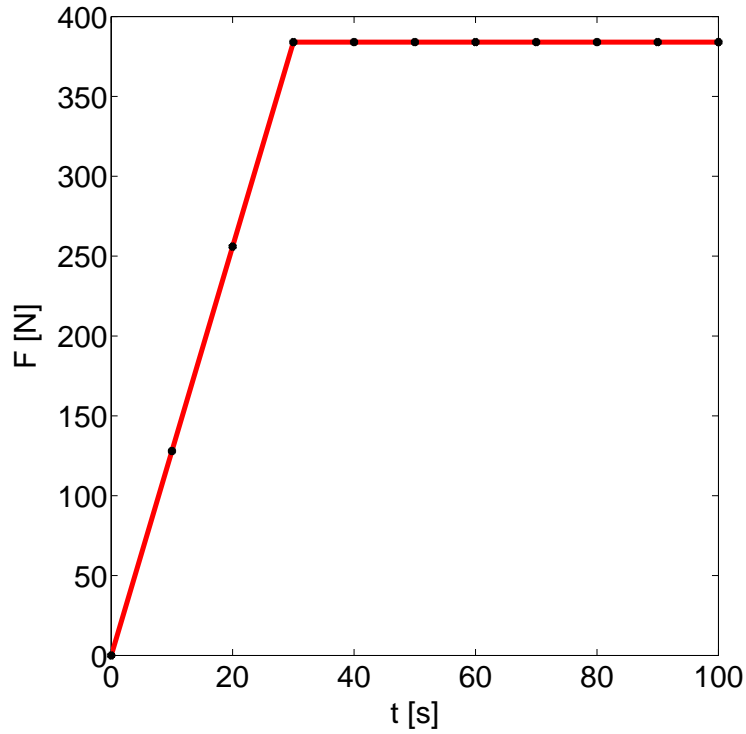

Figure 6.6: Force versus time for the simulation of the measurement data and the simulation within the identification. 
The parameter identification is carried out for the viscoelastic material with one relaxation term $(N=1$ in equation (3.8)). Thereby, within the equilibrium term of the free energy function $\Psi^{\mathrm{eq}}$ - as defined in equation $(3.60)$ - the part $\left[\Psi^{\mathrm{eq}}\right]^{\mathrm{dev}}$ consists of one term, so that $\left(N_{T}=1\right)$ holds. For the one non-equilibrium part $\Psi^{k=1}$ the number of terms in $\left[\Psi^{\mathrm{k}=1}\right]^{\mathrm{dev}}$ is given by $\mathrm{N}_{\mathrm{T}}^{k=1}=1$ as well, so that the corresponding parameter set is given by $\boldsymbol{\kappa}=\left[\left[\mu_{\text {eq }}\right]_{1},\left[\alpha_{\text {eq }}\right]_{1}, \kappa_{\text {eq }},\left[\mu_{\text {neq }}^{\mathrm{k}=1}\right]_{1},\left[\alpha_{\text {neq }}^{\mathrm{k}=1}\right]_{1}, \kappa_{\text {neq }}, \widehat{\tau}^{\mathrm{k}=1}\right]^{\mathrm{T}}$.

The objective function for the inverse problem yields

$$
f(\boldsymbol{\kappa})=\frac{1}{2} \sum_{i=1}^{T} \sum_{j=1}^{N_{g}}\left[\overline{\boldsymbol{u}}_{i j}(\boldsymbol{\kappa})-\overline{\boldsymbol{u}}_{i j}^{\exp }\right]^{2}
$$

and is minimized by means of the Levenberg-Marquardt method. $T$ is the number of load steps with $T=10$ and $N_{g}$ is the number of identification nodes. The five $\left(N_{g}=5\right)$ identification nodes are marked in figure (6.1). The three-dimensional displacements $\overline{\boldsymbol{u}}_{i j}(\boldsymbol{\kappa})$ are simulated by the viscoelastic material described above with seven parameters. In table (6.4) and figure (6.7) the iteration is shown in which after eight iteration steps a local or maybe even global minimum is determined. Since the solution is stable it

Table 6.4: Iteration for the identification for finite viscoelasticity with one relaxation term.

\begin{tabular}{|c||l|l|l|l|l|l|l||c|}
\hline$n$ & {$\left[\mu_{\text {eq }}\right]_{1}$} & {$\left[\alpha_{\text {eq }}\right]_{1}$} & $\kappa_{\text {eq }}$ & {$\left[\mu_{\text {neq }}^{\mathrm{k}=1}\right]_{1}$} & {$\left[\alpha_{\text {neq }}^{\mathrm{k}=1}\right]_{1}$} & $\kappa_{\text {neq }}^{\mathrm{k}=1}$ & $\widehat{\tau}^{\mathrm{k}=1}$ & $f(\boldsymbol{\kappa})$ \\
\hline \hline 0 & 14 & 1.5 & 14 & 14 & 1.5 & 2 & 15 & 24038.5 \\
\hline 1 & 20 & 1.5 & 23.5236 & 15.4605 & 2.23086 & 5.43559 & 10.232 & 2082.95 \\
\hline 2 & 13.0391 & 2.03173 & 37.3725 & 26.4843 & 2.39137 & 13.2734 & 7.2115 & 866.314 \\
\hline 3 & 6.92452 & 2.95216 & 53.5538 & 36.9208 & 2.17134 & 31.2128 & 7.3423 & 625.218 \\
\hline 4 & 5.81038 & 3.73383 & 65.6918 & 39.6741 & 2.19011 & 59.3563 & 7.8656 & 48.7199 \\
\hline 5 & 4.97352 & 4.32744 & 80.3276 & 44.6039 & 1.9545. & 64.0535 & 7.8020 & 5.52809 \\
\hline 6 & 4.93564 & 4.43388 & 86.2499 & 46.6722 & 1.91372 & 55.2726 & 7.6610 & 0.08398 \\
\hline 7 & 4.93197 & 4.43925 & 86.9473 & 46.3039 & 1.93621 & 52.0508 & 7.6357 & 0.06743 \\
\hline 8 & 4.93157 & 4.4395 & 86.9894 & 46.335 & 1.93617 & 51.6013 & 7.6308 & 0.06741 \\
\hline 9 & 4.93149 & 4.43954 & 86.9949 & 46.3377 & 1.93619 & 51.5454 & 7.6302 & 0.06741 \\
\hline 10 & 4.93147 & 4.43954 & 86.9959 & 46.3401 & 1.93612 & 51.5342 & 7.6301 & 0.06741 \\
\hline & {$[\mathrm{MPa}]$} & {$[-]$} & {$[\mathrm{MPa}]$} & {$[\mathrm{MPa}]$} & {$[-]$} & {$[\mathrm{MPa}]$} & {$[\mathrm{s}]$} & {$\left[\mathrm{mm}^{2}\right]$} \\
\hline
\end{tabular}

might be assumed that the global minimum has been found. In addition figure (6.8) shows - for the example of the tenth time step - that the deformed FE models for the virtual measurement data and for the solution of the identification problem optically (almost) coincide. Moreover, as presented in figure (6.11) for different load steps both FE simulations have almost the same distribution of stresses (here: euclidean norm of the viscous part of the Cauchy stresses).

Also for the identification of large strain viscoelasticity based on Ogden models the results show that the necessity of the use of multiple terms within the sum for the respective deviatoric part of the energy might not be given for the later parameter identification for real materials. Therefore - as well as in order to avoid instabilities due to overparametrization and in order to save computational costs - within the viscoelastic material law only one term for each deviatoric part of the energy will be used $\left(\mathrm{N}_{T}=\mathrm{N}_{\mathrm{T}}^{k}=1 \forall k\right)$. 


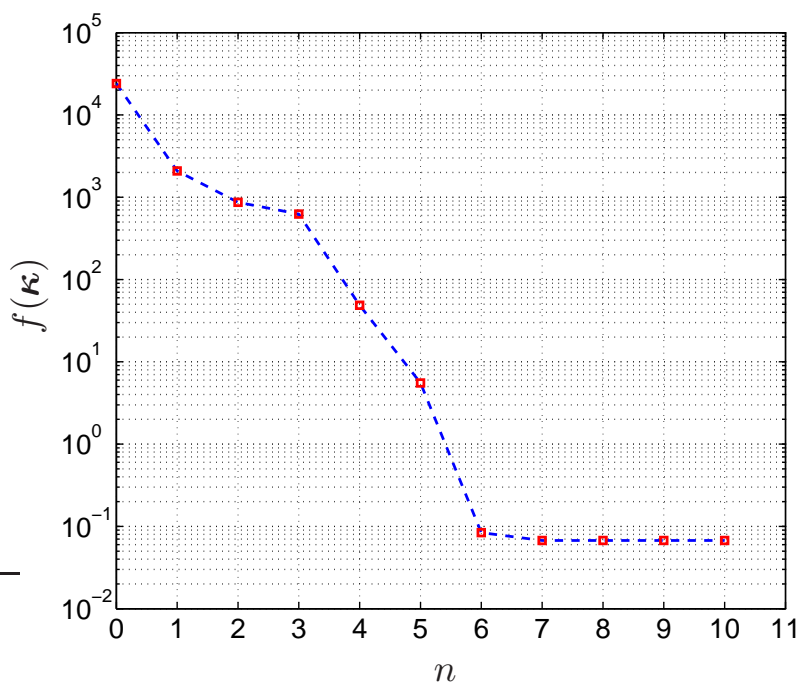

Figure 6.7: Sum of squared differences versus iteration steps for the identification of finite viscoelasticity.

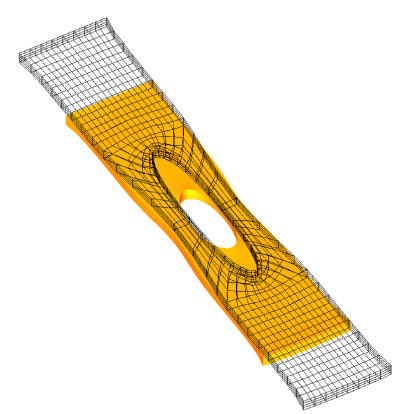

(a) Start of the identification iteration $(n=0)$.

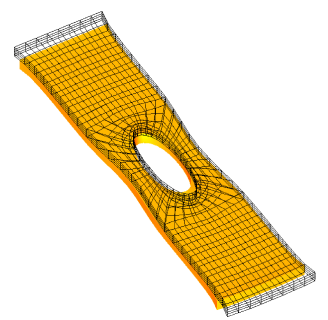

(d) After three iteration steps.

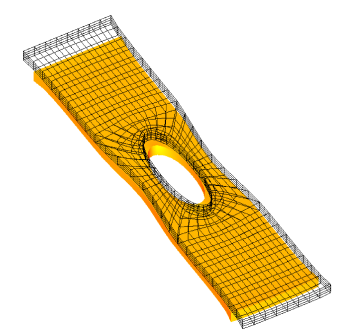

(b) After one iteration step.

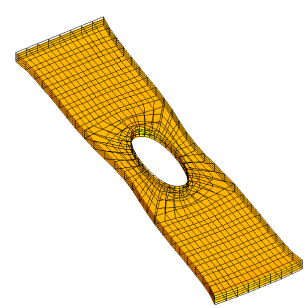

(e) After four iteration steps.

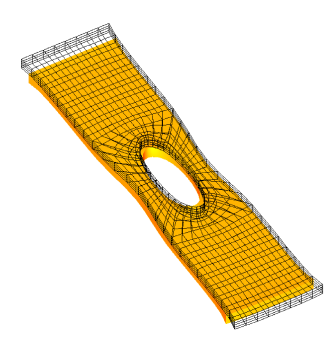

(c) After two iteration steps.

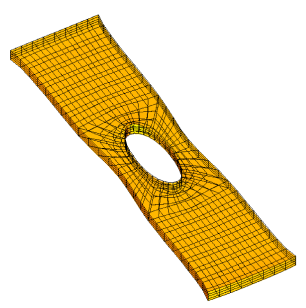

(f) After six iteration steps.

Figure 6.8: FE model of the viscoelastic material with seven parameters over the virtual specimen during the iteration at the tenth time step. 


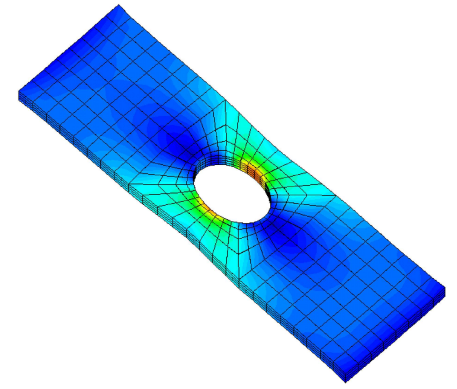

(a) Second time step.

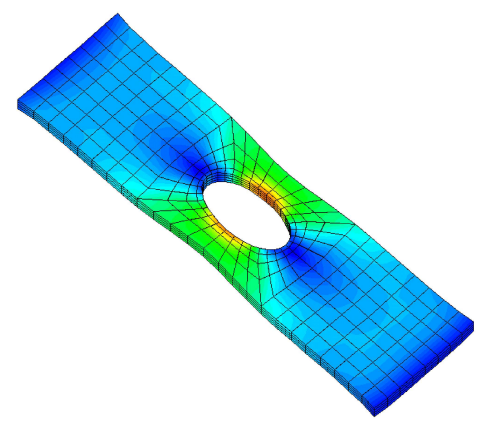

(b) Fourth time step.

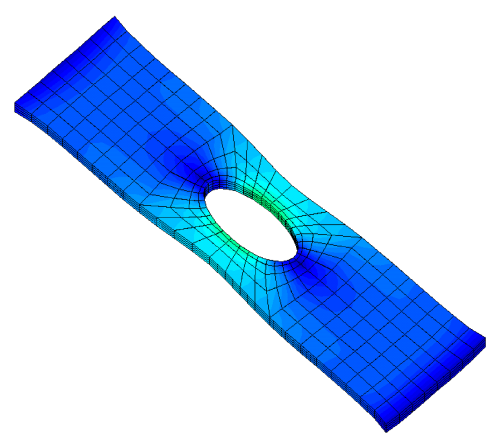

(c) Sixth time step.

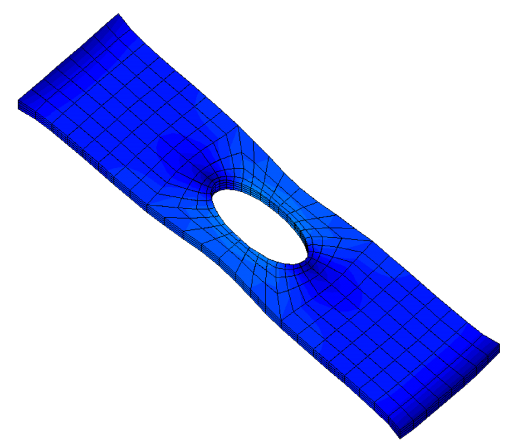

(d) Eighth time step.

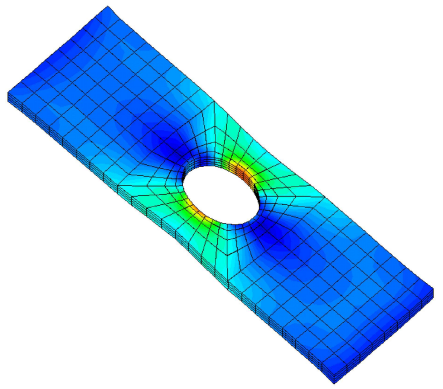

(a) Second time step.

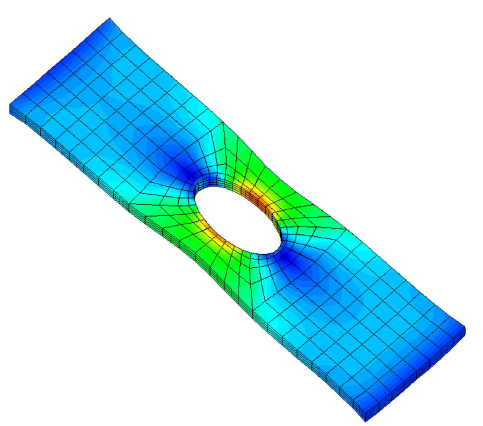

(b) Fourth time step.

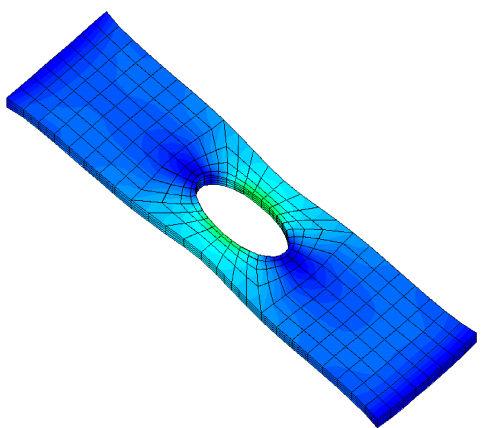

(c) Sixth time step.

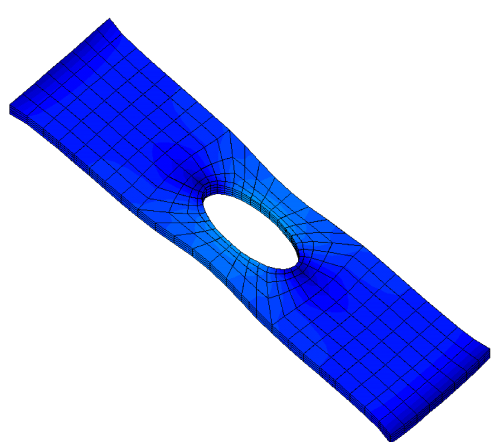

(d) Eighth time step.
Figure 6.9: FE simulation for the virtual measurement data (15 parameters).
Figure 6.10: FE simulation for the identified material parameters (7 parameters) after six iteration steps.

Figure 6.11: Comparison of the euclidian norm of the viscous part of the Cauchy stresses. 


\section{Parameter identification for finite viscoelasticity on the basis of real measurement data}

In this chapter the experimental observations on different elastomeric materials and the respective parameter identification for the finite viscoelastic material (see chapter (3)) are described. In section 7.1 the parameter identification for a polyurethane adhesive is considered, whereby only the short time viscoelastic behavior - with one relaxation term for the material model - shall be investigated by taking into account the experimental data of a single relaxation test. The parameter estimation for a cellular polyurethane follows in section 7.2 , whereby we aim at identifying the parameters of the viscoelastic law with one relaxation term by means of three different experimental test programs. In order to describe the viscoelastic behavior of a compact polyurethane universally for short and long time loads, in section (7.3) the parameter identification for two short- and one long time tests is conducted.

Note, that within the experiments the influence of damage effects has to be excluded. A typical damage effect of elastomeric materials is the Mullins effect. This strain-induced stress softening is occurring mainly in the first load cycle. In order to cause a saturation of damage, before the conduction of the first test each specimen is loaded in a number of load cycles with the maximal displacement used in the later tests. The number of necessary load cycles depends on the kind of elastomer, especially on its chemical composition. Sufficiently long before the conduction of each test, the specimen is not loaded in order to exclude relaxation effects of the previous test.

\subsection{Polyurethane adhesive}

The simulation of strength difference for adhesive materials in finite deformation elastoplasticity has recently been investigated by Mahnken [44] and Mahnken and Schlimmer [46]. Considering an adhesive material with viscoelastic material behavior, in this section the parameter identification for the polyurethane adhesive with the trade name KÖRAPUR $125^{1}$ is presented. This one component polyurethane which is fully curing with humidity is used as adhesive and sealant for, e.g. primered and varnished metals, aluminum and wooden materials. The cured adhesive has, referring to the data sheet, a density of approx. $1.2 \mathrm{~g} / \mathrm{cm}^{3}$, a Shore A hardness of approx. 45 and a very high elongation at tear of circa $450 \%$.

By verification it can be shown that the material model with the identified parameters

${ }^{1}$ KÖMMERLING CHEMISCHE FABRIK GMBH 
delivers a very good agreement between experimental and simulated data for the investigated test.

\subsubsection{Experiments}

The experimental data was determined by D. Vogt at the 'Work Group for Materials and Surface Technology (AWOK)', Jun.-Prof. Dr.-Ing. P. Geiß, Technical University of Kaiserslautern.

For the manufacture of a plate of the cured adhesive, the pasty material has been applied with a blade and a putty gun to a substrate holder. The plate has a thickness of $1.5 \mathrm{~mm}$, is $25 \mathrm{~mm}$ wide and has a clamping length of $60 \mathrm{~mm}$. For the later identification the test has to deliver sufficient information about the deformation behavior of the material. Thus for the specimen a geometry is to be chosen which immediately leads to inhomogeneous displacement fields in a tensile test. For this reason a hole of $10 \mathrm{~mm}$ is punched in the middle of the cured specimen.

The tensile test is conducted in force control. In the photography in figure 7.1 the specimen is shown whereby for the optical deformation analysis by means of video extensometry gauge marks have been applied onto the specimen's surface (see also section 4.2). In

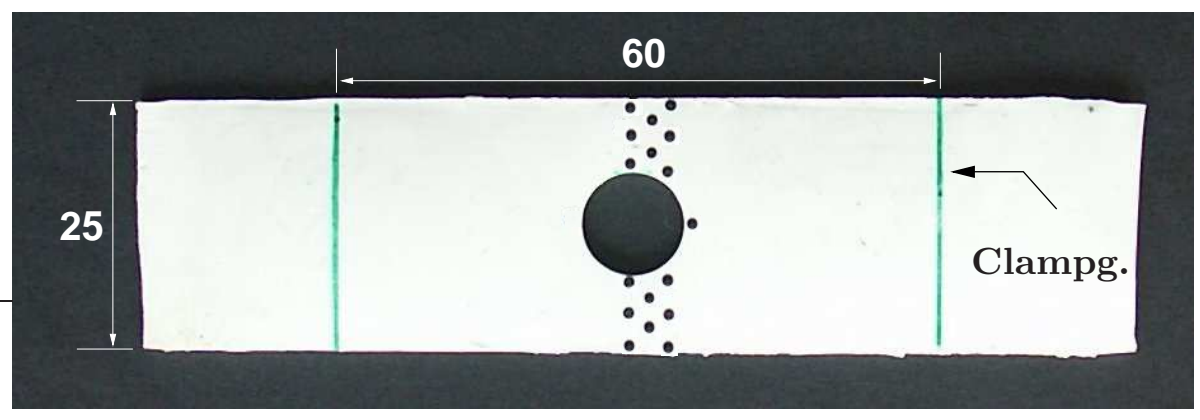

Figure 7.1: Specimen with gauge marks for video extensometry measurement.

analogy to the picture in figure 7.2 the far ends of the specimen are clamped between steel plates of a tensile testing machine which is driven by an engine of variable speed and rolled ball screws. The CCD camera of the measurement system is positioned perpendicular to the measured surface of the specimen.

The test data are the force-time curve and the two-dimensional displacement field on the surface of the specimen. The respective measurements are synchronized whereby every $\Delta t^{m s}=0.3 s$ the force is measured and an image of the specimen is taken by the CCD cameras for the displacement measurement. By means of photogrammetric evaluation procedures for the image processing, the measuring system evaluates the two-dimensional displacement field associated with each measured time step. The corresponding force-time curve is shown in figure 7.3, whereby $F$ is the total force in loading direction.

\subsubsection{Interpolation of experimental data}

For the simulation of the tests four FEM discretizations of the specimen with 48, 192, 432 and 2400 eight-node standard displacement-based elements (Q1) are used whereby 


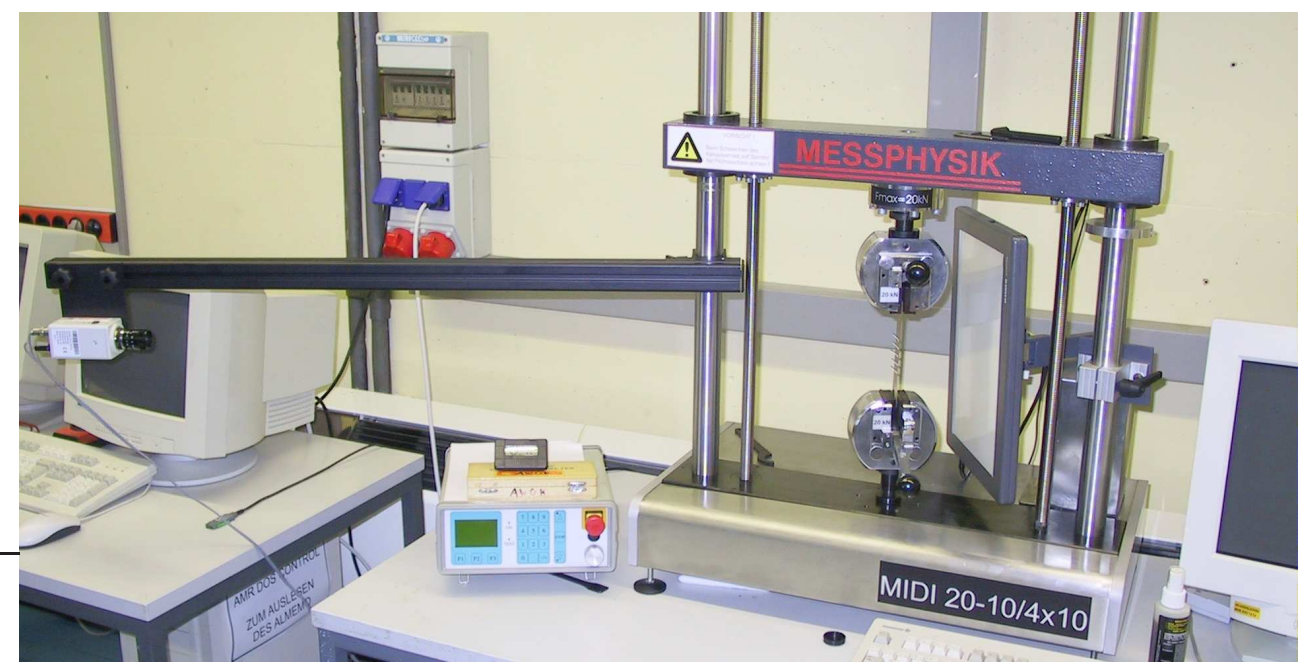

Figure 7.2: $C C D$ camera for video extensometry in a tensile test.

for the first three models the whole specimen is discretized. For the model with 2400 elements symmetry conditions in transverse direction are applied and hence only half of the width is discretized. The boundary conditions for both ending planes normal to the longitudinal direction are chosen in correspondence with the experiments whereby the nodes on one plane are fixed in longitudinal and transverse direction. The nodes on the other plane are fixed in transverse direction and in longitudinal direction uniaxial normal forces are applied whereby warping of the plane is prevented. In addition, for both planes the edges and straight lines through the nodes along the transverse direction are prevented from curvature and hence can only move parallel in thickness direction. Rigid body motion is excluded. Figure 7.4 shows the entire FEM mesh with the discretization of the respective corresponding FEM model with 2400 elements. In order to reduce the computational costs for the identification routine these FEM discretizations are used in ascending order within a multi level strategy (see Mahnken and Stein [47]). The optimal material parameters determined with the 48-element model at level one are used as initial parameters for the optimization based on the 192-element model at level two and so forth.

Since the coordinates of the measurement points generally do not coincide with the coordinates of the identification nodes, the measured displacements are interpolated linearly on the identification nodes by triangulation (see section 4.3). For the triangulation the displacements of the three measurement points being nearest to the respective identification node are used. As only half of the width is discretized for the FE model of level four, the measured displacements of the opposite side of the longitudinal centerline are mirrored onto the considered side and two separate triangulations for both displacement fields on the identification nodes are performed. For each identification node and displacement direction the mean value of these two data sets is taken into account. 


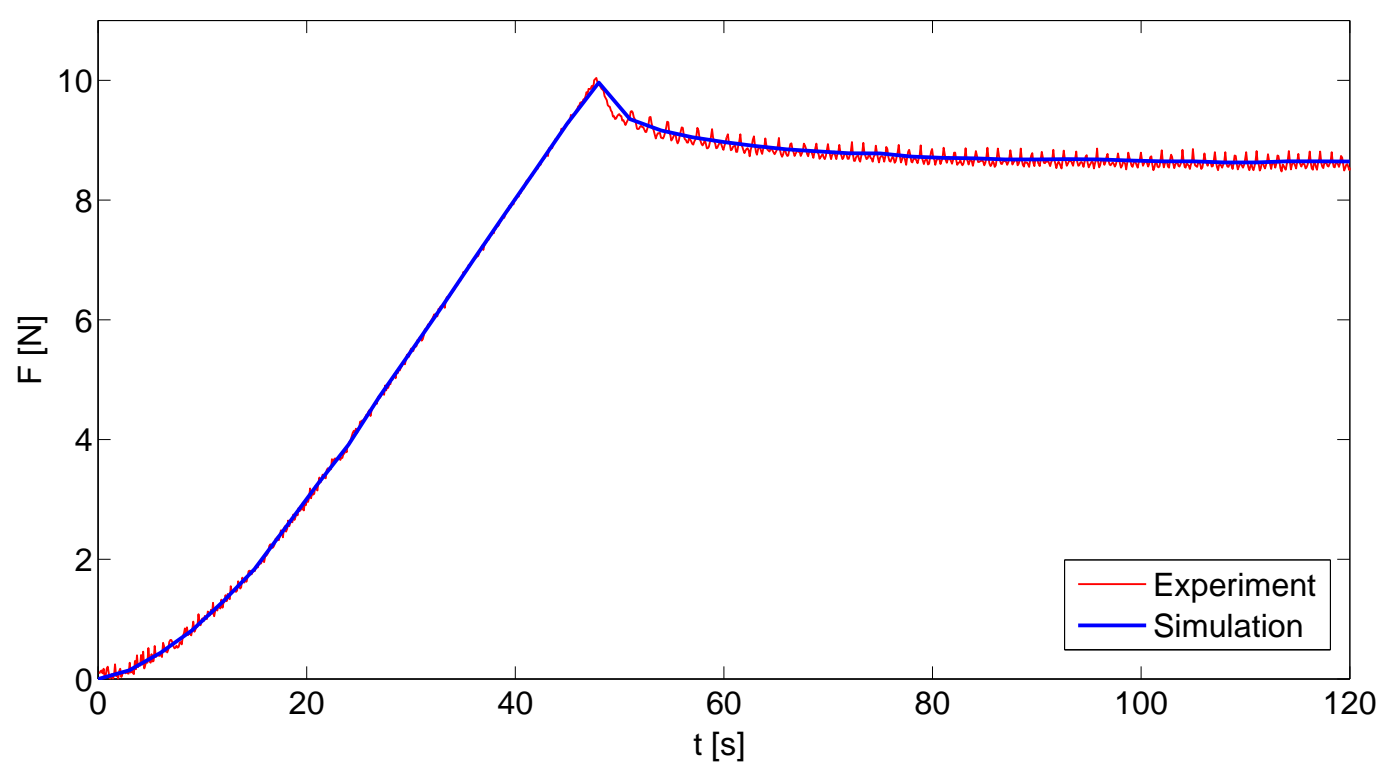

Figure 7.3: Force versus time curve in experiment and for the force control of the identification simulation.

\subsubsection{Choice of the model structure and parameter identification process}

Since the test shows that the material exhibits one defined relaxation process in the considered time range, the identification is carried out for the viscoelastic material with one relaxation term $(N=1$ in equation (3.8)). Thereby, within the equilibrium term of the free energy function $\Psi^{\mathrm{eq}}$ - as defined in equation $(3.60)$ - the part $\left[\Psi^{\mathrm{eq}}\right]^{\mathrm{dev}}$ consists of one term, so that $\left(N_{T}=1\right)$ holds. For the one non-equilibrium part $\Psi^{k=1}$ the number of terms in $\left[\Psi^{\mathrm{k}=1}\right]^{\mathrm{dev}}$ is given by $\mathrm{N}_{\mathrm{T}}^{k=1}=1$ as well ${ }^{2}$, so that the corresponding parameter set is given by $\boldsymbol{\kappa}=\left[\left[\mu_{\text {eq }}\right]_{1},\left[\alpha_{\text {eq }}\right]_{1}, \kappa_{\text {eq }},\left[\mu_{\text {neq }}^{\mathrm{k}=1}\right]_{1},\left[\alpha_{\text {neq }}^{\mathrm{k}=1}\right]_{1}, \kappa_{\text {neq }}^{\mathrm{k}=1}, \hat{\tau}^{\mathrm{k}=1}\right]^{\mathrm{T}}$.

As mentioned before the time intervals for the measurements of the forces and displacements are $\Delta t^{m s}=0.3 \mathrm{~s}$. The total time for the test is $120 \mathrm{~s}$. Numerical pre studies have been conducted in order to determine a sufficient time step length for the viscoelastic material law with one relaxation term in combination with the considered time dependent material behavior in the experiment. It turned out that a time step length of $\Delta t=3 \mathrm{~s}$ is small enough to guarantee a sufficiently accurate computation of the viscous internal variable within the material law for the (up to now estimated bandwidth of the) material parameters. Hence the number of load steps for the simulation of the test yields $T=40$. The objective function for the inverse problem yields

$$
f(\boldsymbol{\kappa})=\frac{1}{2} \sum_{i=1}^{T} \sum_{j=1}^{N_{g}}\left[\overline{\boldsymbol{u}}_{i j}(\boldsymbol{\kappa})-\overline{\boldsymbol{u}}_{i j}^{\text {exp }}\right]^{2} .
$$

\footnotetext{
${ }^{2}$ As described in subsection 6.2 the implementation of more than one term will not lead to a significant improvement (lowering of the sum of squared differences) but might lead to an instable or non-unique solution for the identified material parameters. In addition, the parameters for the extra terms implicate extra computational costs.
} 


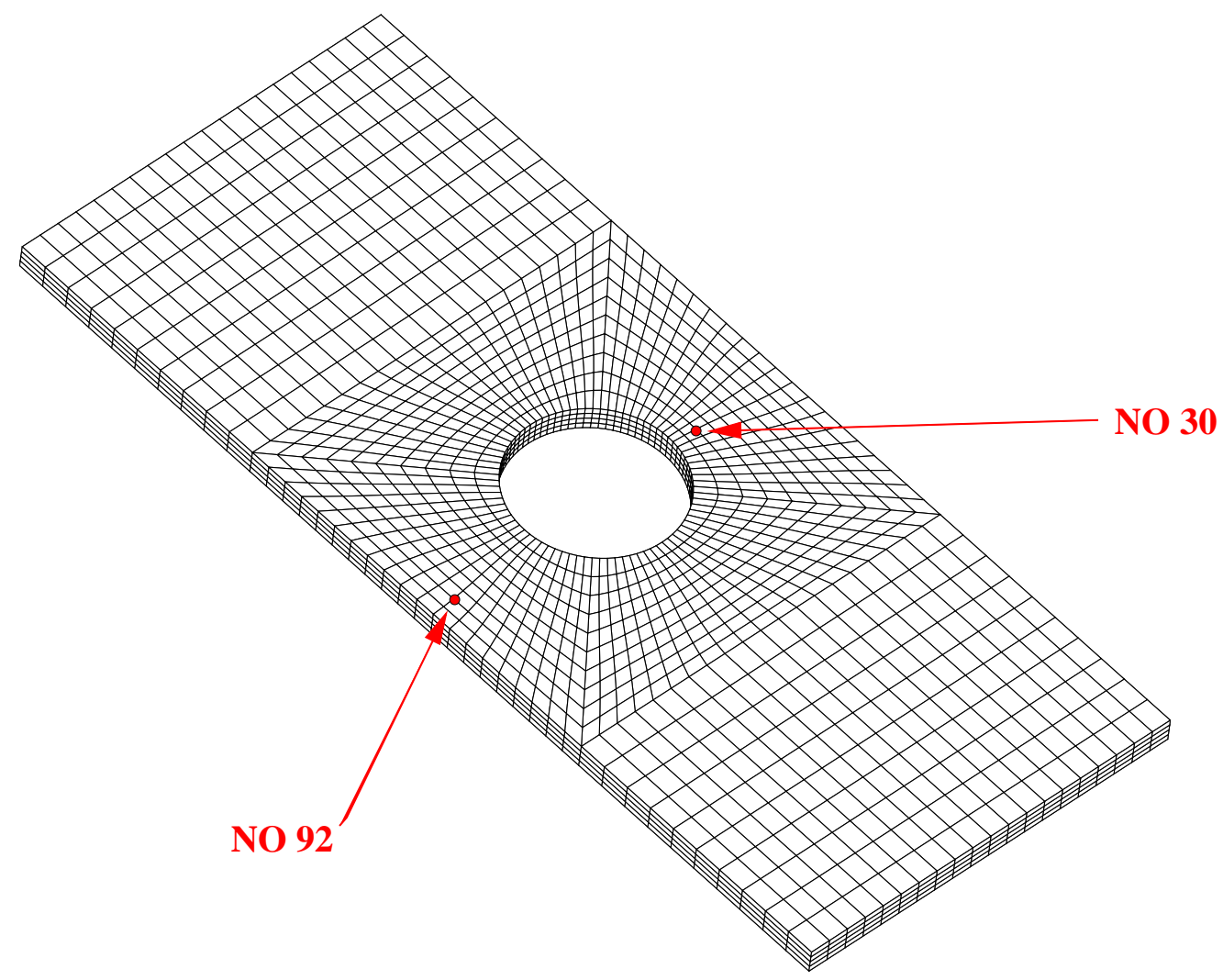

Figure 7.4: Entire FEM model with the discretization of the respective corresponding FEM model with 2400 elements; Identification nodes NO 30 and NO 92.

For the simulation of the displacement fields $\overline{\boldsymbol{u}}_{i j}(\boldsymbol{\kappa})$ the FEM simulation is force-controlled based on the measured force signals shown in figure 7.3. Moreover, $N_{g}$ denotes the number of identification nodes which is dependent on the discretization for the different $\mathrm{FE}$ models in the identification process as described in subsection 7.1.2. For level four the discretization yields $N_{g}=N_{g}^{l e v_{4}}=59$.

The displacements calculated by FEM $\overline{\boldsymbol{u}}_{i j}(\boldsymbol{\kappa})$ and the measured displacements $\overline{\boldsymbol{u}}_{i j}^{\text {exp }}$ have, respectively, two displacement directions. Thus for the FEM discretization with 2400 elements the number of terms in the least square sum adds up to $59 \times 40 \times 2=4720$ per iteration step of the optimization algorithm. By taking into account that the displacement data of the experiments has been mirrored, the total number of displacement data compared within the optimization algorithm is $2 \times 4720=9440$ per iteration step.

Because of the non-convexity of the objective function and the existence of (many) local minima the hybrid method of application of the Levenberg-Marquard method to find different local minima for stochastically chosen start parameter sets is used (see section 4.4). Basically it can be observed that for each new starting point a number of eight iteration steps are enough to detect if the starting point leads to a local minimum or 
whether this iteration can be discarded because of divergence. In order to save computing time the above described hybrid method is mainly applied in the first and second level, whereby the FEM models with 48 and 192 elements are used for the identification routine. The start parameter set leading to the smallest of all investigated local minima is shown in table 7.1 .

Table 7.1: Start parameter set.

\begin{tabular}{|l|l|l|l|l|l|l|}
\hline$\left[\mu_{\text {eq }}\right]_{1}$ & {$\left[\alpha_{\text {eq }}\right]_{1}$} & $\kappa_{\text {eq }}$ & {$\left[\mu_{\text {neq }}^{\mathrm{k}=1}\right]_{1}$} & {$\left[\alpha_{\text {neq }}^{\mathrm{k}=1}\right]_{1}$} & $\kappa_{\text {neq }}^{\mathrm{k}=1}$ & $\hat{\tau}^{\mathrm{k}=1}$ \\
\hline \hline 0.5 & 1.0 & 10 & 0.5 & 1.0 & 1.0 & 20 \\
\hline$[\mathrm{MPa}]$ & {$[-]$} & {$[\mathrm{MPa}]$} & {$[\mathrm{MPa}]$} & {$[-]$} & {$[\mathrm{MPa}]$} & {$[\mathrm{s}]$} \\
\hline
\end{tabular}

Note that in contrast to the parameter identification for, e.g. hyperelasticity, the identification of time dependent problems is generally more complex. One the one hand the extra effort is due to the increased number of material parameters, which make the simultaneous identification for all parameters increasingly difficult due to instabilities. On the other hand the identification of the relaxation time is complicated since it is strongly affecting all other parameters. If $\hat{\tau}^{\mathrm{k}=1}$ is increasing, the non-equilibrium term of the energy will increasingly behave like an extra equilibrium term which can be confused with the original equilibrium term in the identification process. In contrast, if $\hat{\tau}^{\mathrm{k}=1}$ becomes too small the non-equilibrium term will vanish too quickly. Then the identification algorithm could use this term in order to fit the simulation even better to a part of the deformation in which no distinction between the pure elastic and the pure viscous part of the deformation can be determined. This might happen, if a constitutive routine does not fit adequate neither in the elastic nor in the viscous regime of the material behavior and moreover the experiments do not (also) have distinctive relaxation or creep processes. Only if the material model has the capability to be fitted to the considered real material and the conducted experiment is appropriate, reliable results for the relaxation time affecting the whole behavior of the material simulation - can be determined.

In order to underline the necessity of time ranges within the experiments which only serve for the distinction between the viscous and the elastic parameters, figures 7.5 and 7.6 demonstrate the simulated material behavior for one of the starting points of the identification iteration. For representation - in order to compare the results for the starting point to the later verification, which is conducted on the basis of the identified parameters - the simulation for the starting point is carried out for the FE discretization of the fourth level, too. The corresponding identification nodes are marked in figure 7.4, whereby the identification node NO 30 is belonging to the number of identification nodes $\left(N_{g}=N_{g}^{l e v_{4}}=59\right)$ for half of the FE model (with 2400 elements) and identification node NO 92 has been mirrored back for the whole discretization with 118 identification nodes for representation. Although the simulation for the first fourty seconds of the test (during the beginning of the loading) already delivers good agreement to the experimental observations, the following creep of the specimen does not give any accordance. Moreover, an elastomeric material always exhibits several different superposed relaxation effects within the long- and several short time ranges, which could only be simulated by several relaxation mechanisms, and the parameters of which could only be identified in combination with several tests in these time ranges. Therefore, it needs to be mentioned that the equi- 
librium parameters to be determined within this identification only present parameters for an 'pseudo'-equilibrium state, referring to a time range not (very) much longer than the time range of the considered experiment.
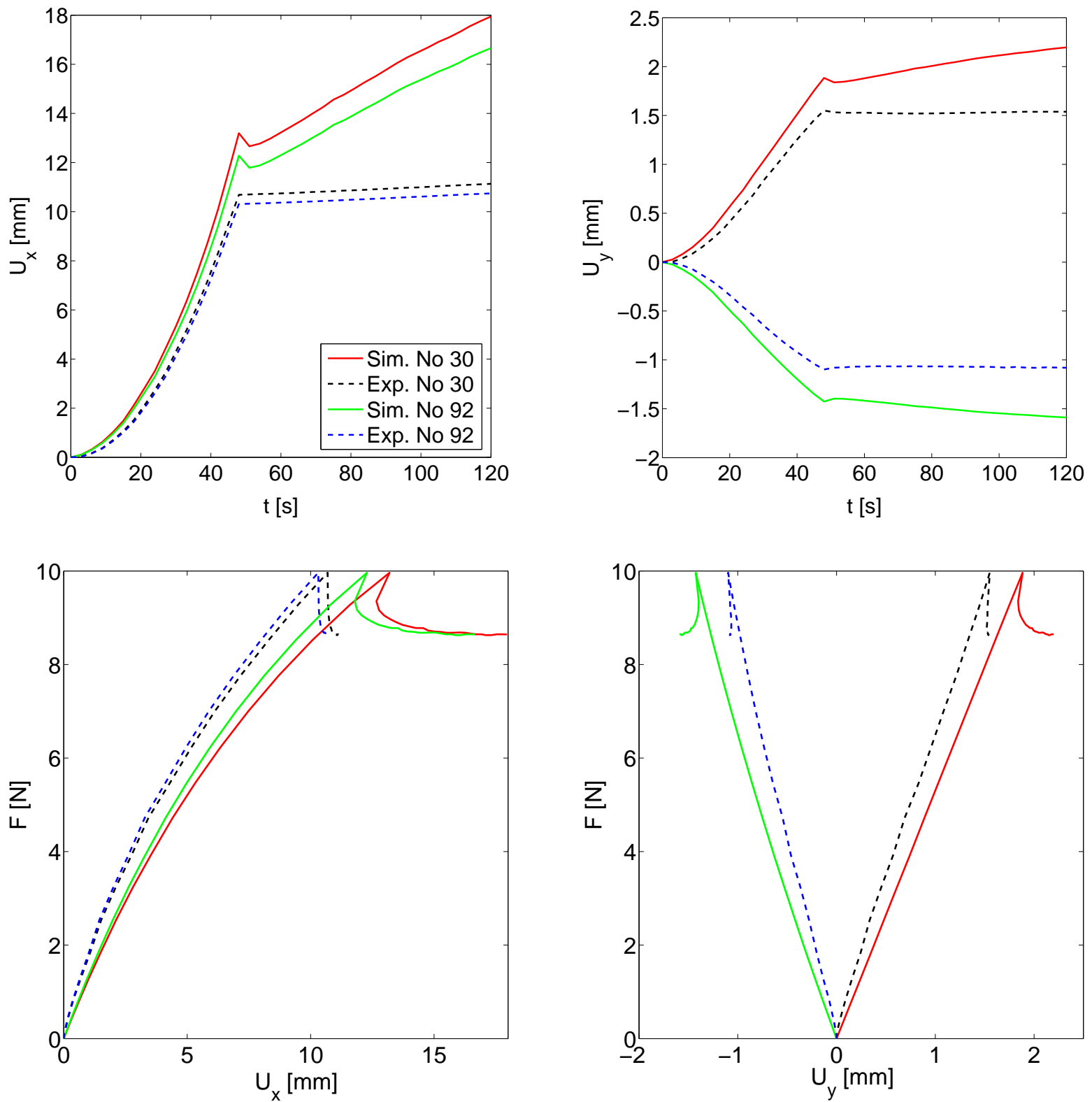

Figure 7.5: Start of the identification iteration. Displacement versus time curves and force versus displacement curves for two representative identification nodes for the experimental and simulated data. 


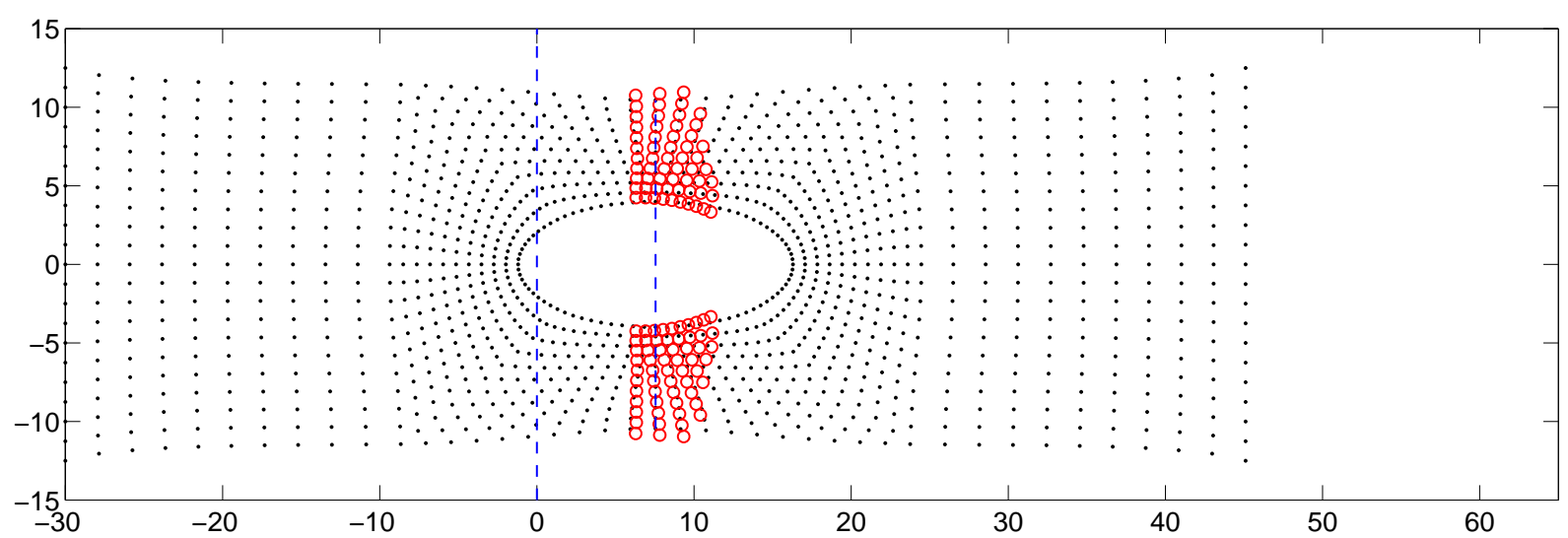

(a) 13th time step.

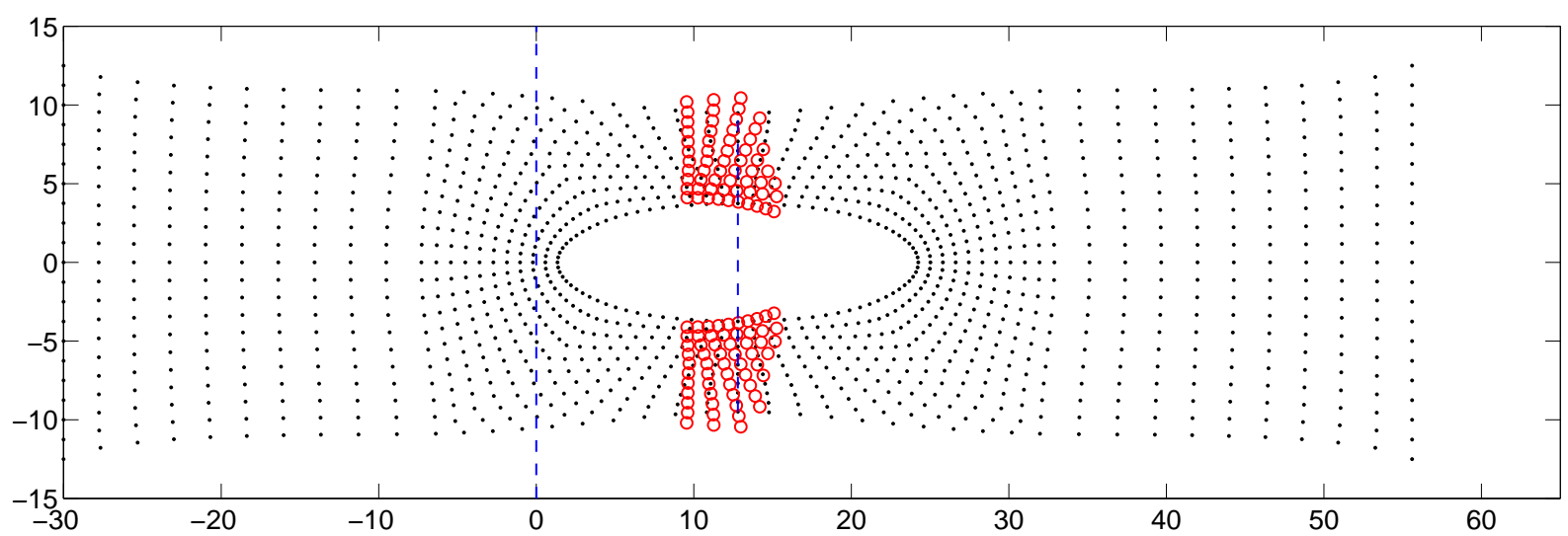

(b) 27 th time step.

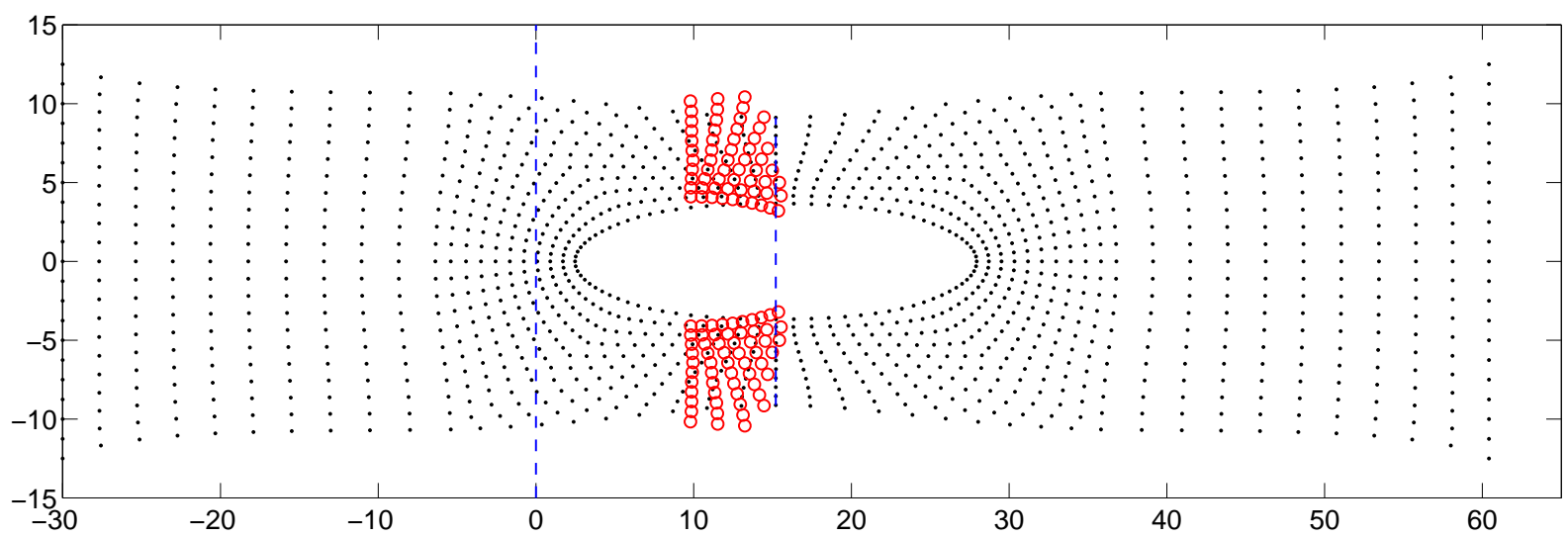

(c) 40th time step.

Figure 7.6: Starting point of the iteration: Interpolated measured displacements (red) over the identification nodes. 
The set of material parameters with the smallest sum of squared differences - chosen out of all determined local minima and therefore denominated 'optimal' - is listed in table 7.2 .

Table 7.2: Parameter set with 'optimal' material parameters.

\begin{tabular}{|l|l|l|l|l|l|l|}
\hline$\left[\mu_{\text {eq }}\right]_{1}$ & {$\left[\alpha_{\text {eq }}\right]_{1}$} & $\kappa_{\text {eq }}$ & {$\left[\mu_{\text {neq }}^{\mathrm{k}=1}\right]_{1}$} & {$\left[\alpha_{\text {neq }}^{\mathrm{k}=1}\right]_{1}$} & $\kappa_{\text {neq }}^{\mathrm{k}=1}$ & $\hat{\tau}^{\mathrm{k}=1}$ \\
\hline \hline 0.317966 & 2.32189 & 2.03756 & 1.14988 & 1.29458 & 0.888432 & 2.17529 \\
\hline$[\mathrm{MPa}]$ & {$[-]$} & {$[\mathrm{MPa}]$} & {$[\mathrm{MPa}]$} & {$[-]$} & {$[\mathrm{MPa}]$} & {$[\mathrm{s}]$} \\
\hline
\end{tabular}

It needs to be mentioned that due to instabilities in the identification iteration there exist several parameter sets which lead to a insignificantly higher least squares sum. In order to evaluate the significance for the quality of the solution, the correlation matrix for the optimal parameters shown in table 7.3 (see also equations (4.15) and (4.16)) is considered. The absolute values of most of the correlation numbers are small. There are

Table 7.3: Correlation matrix for the set of optimal parameters at level two.

\begin{tabular}{|c||l|l|l|l|l|l|l|}
\hline & {$\left[\mu_{\text {eq }}\right]_{1}$} & {$\left[\alpha_{\text {eq }}\right]_{1}$} & $\kappa_{\text {eq }}$ & {$\left[\mu_{\text {neq }}^{\mathrm{k}=1}\right]_{1}$} & {$\left[\alpha_{\text {neq }}^{\mathrm{k}=1}\right]_{1}$} & $\kappa_{\text {neq }}^{\mathrm{k}=1}$ & $\hat{\tau}^{\mathrm{k}=1}$ \\
\hline \hline$\left[\mu_{\mathrm{eq}}\right]_{1}$ & 1.000 & -0.997 & -0.205 & 0.189 & -0.195 & 0.253 & 0.190 \\
\hline$\left[\alpha_{\mathrm{eq}}\right]_{1}$ & -0.997 & 1.000 & 0.126 & -0.173 & 0.178 & -0.225 & -0.187 \\
\hline$\kappa_{\mathrm{eq}}$ & -0.205 & 0.126 & 1.000 & -0.180 & 0.184 & -0.407 & -0.172 \\
\hline$\left[\mu_{\text {neq }}^{\mathrm{k}=1}\right]_{1}$ & 0.189 & -0.173 & -0.180 & 1.000 & -1.000 & 0.390 & -0.595 \\
\hline$\left[\alpha_{\text {neq }}^{\mathrm{k}=1}\right]_{1}$ & -0.195 & 0.178 & 0.184 & -1.000 & 1.000 & -0.401 & 0.585 \\
\hline$\kappa_{\text {neq }}^{\mathrm{k}=1}$ & 0.253 & -0.225 & -0.407 & 0.390 & -0.401 & 1.000 & 0.431 \\
\hline$\hat{\tau}^{\mathrm{k}=1}$ & 0.190 & -0.187 & -0.172 & -0.595 & 0.585 & 0.431 & 1.000 \\
\hline
\end{tabular}

three categories of parameters which correlate.

Firstly, the pairs of parameters $\left(\left[\mu_{\mathrm{eq}}\right]_{1},\left[\alpha_{\mathrm{eq}}\right]_{1}\right)$ and $\left(\left[\mu_{\mathrm{neq}}^{\mathrm{k}=1}\right]_{1},\left[\alpha_{\mathrm{neq}}^{\mathrm{k}=1}\right]_{1}\right)$ have correlation numbers close to \pm 1 . This is due to the structure of the Ogden-model which can lead to overparametrization even for only one deviatoric term within the corresponding part of the energy. Since only parameters within the particular deviatoric terms are affected, these correlations - though the respective parameters are instable in relation to each other will not have a significant influence on the quality of the solution.

Secondly, the parameters for the deviatoric part of the relaxation module have comparatively high correlation numbers in relation to the first relaxation time $\left[\left(\left[\mu_{\text {neq }}^{\mathrm{k}=1}\right]_{1}, \hat{\tau}^{\mathrm{k}=1}\right)\right.$ and $\left.\left(\left[\alpha_{\mathrm{neq}}^{\mathrm{k}=1}\right]_{1}, \hat{\tau}^{\mathrm{k}=1}\right)\right]$. As mentioned before, instabilities in finding one fixed relaxation time are due to the general complex viscoelastic behavior of elastomers. Moreover, the values are still too small to indicate the respective parameters as instable ones.

Remark 7.1.1 The tests might have too few information about the (viscoelastic) behavior under compression (only parts around the hole are loaded under compression). The validity of the identified set of parameters for compression-dominated loading, however, is not investigated in this example but rather constitutes future work.

Remark 7.1.2 Since only one test for one specimen is considered in this study, no conclusion about the validity of the identified parameters can be made. The method of parameter identification for multiple tests, however, will be described in the following examples. 


\section{Verification}

Within the verification procedure the simulated data - calculated as based on the optimal parameters for the FEM model with 2400 elements - is compared with the experimental data used for the parameter identification. By this the quality of the identified parameters and also the general ability of the constitutive model to describe the viscoelastic behavior of the material is analyzed. In particular, two representative identification nodes out of $N_{g}=59$ are chosen. The first is referred to by number 30 and the second has been mirrored back for representation and is referred by number 103 as shown in figure 7.31. The verification shows that a very good qualitative and quantitative agreement between the experiment and the simulation can be observed (see figures 7.7-7.9).
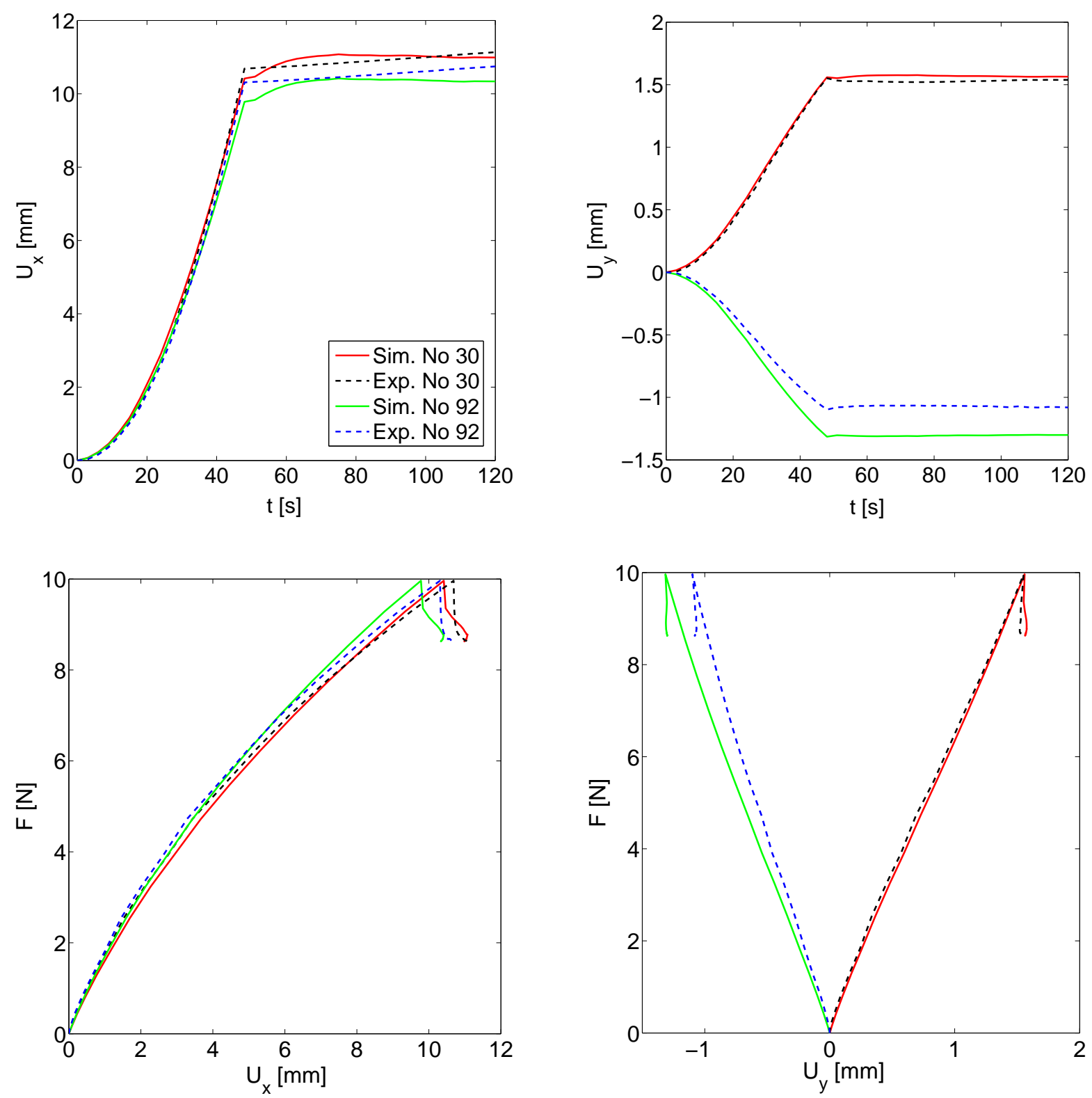

Figure 7.7: End of the identification iteration. Displacement versus time curves and force versus displacement curves for two representative identification nodes for the experimental and simulated data. 


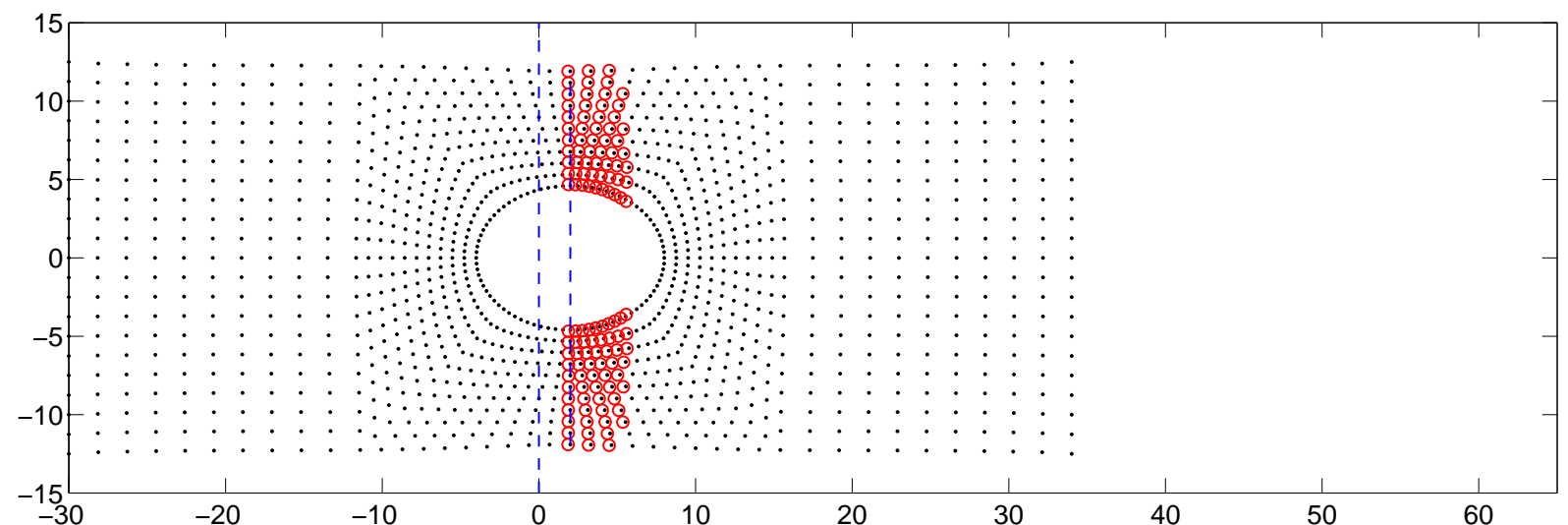

(a) 7 th time step.

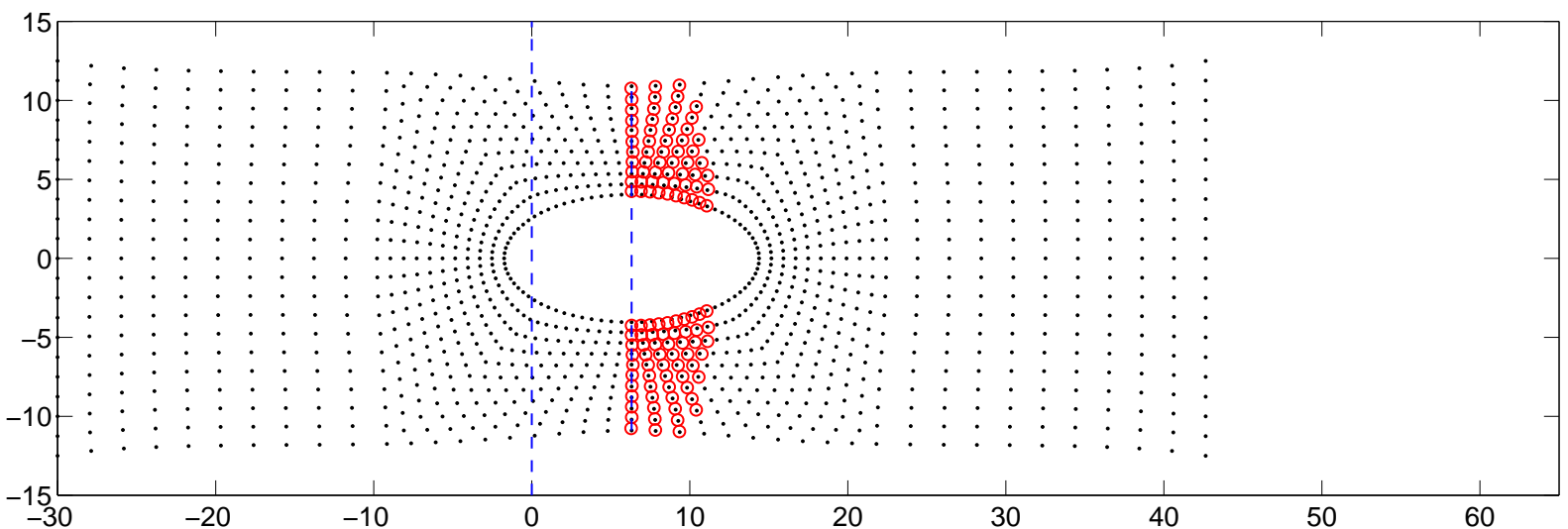

(b) 13th time step.

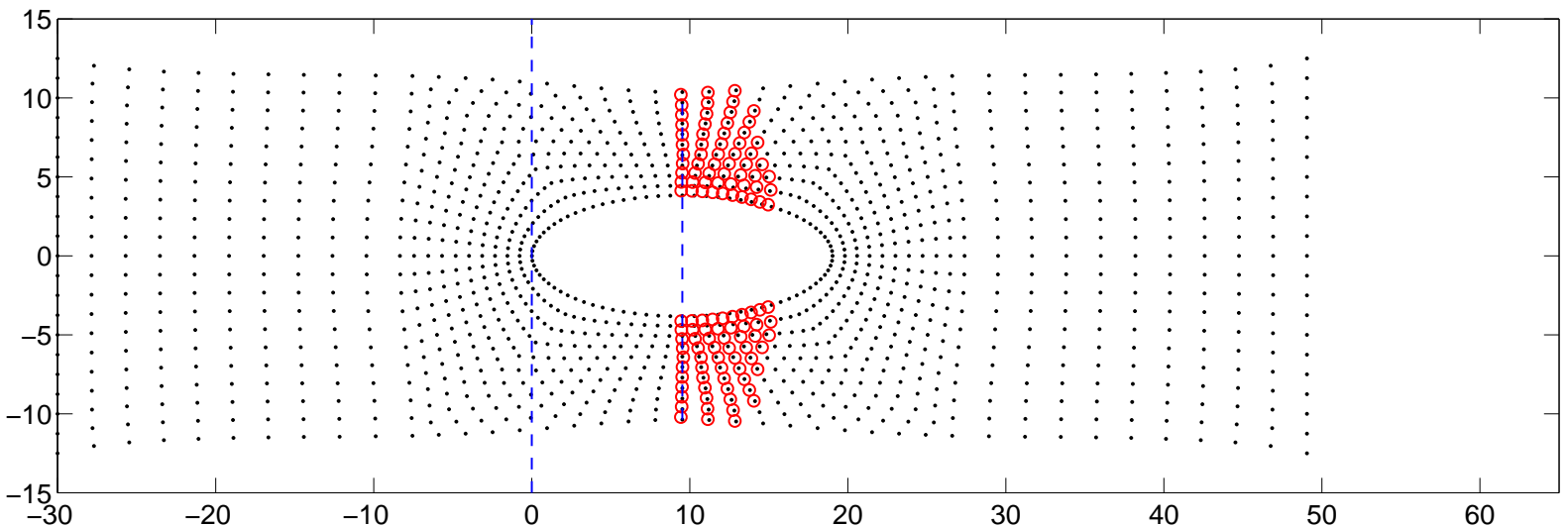

(c) 20th time step.

Figure 7.8: End of the iteration: Interpolated measured displacements (red) over the identification nodes. 


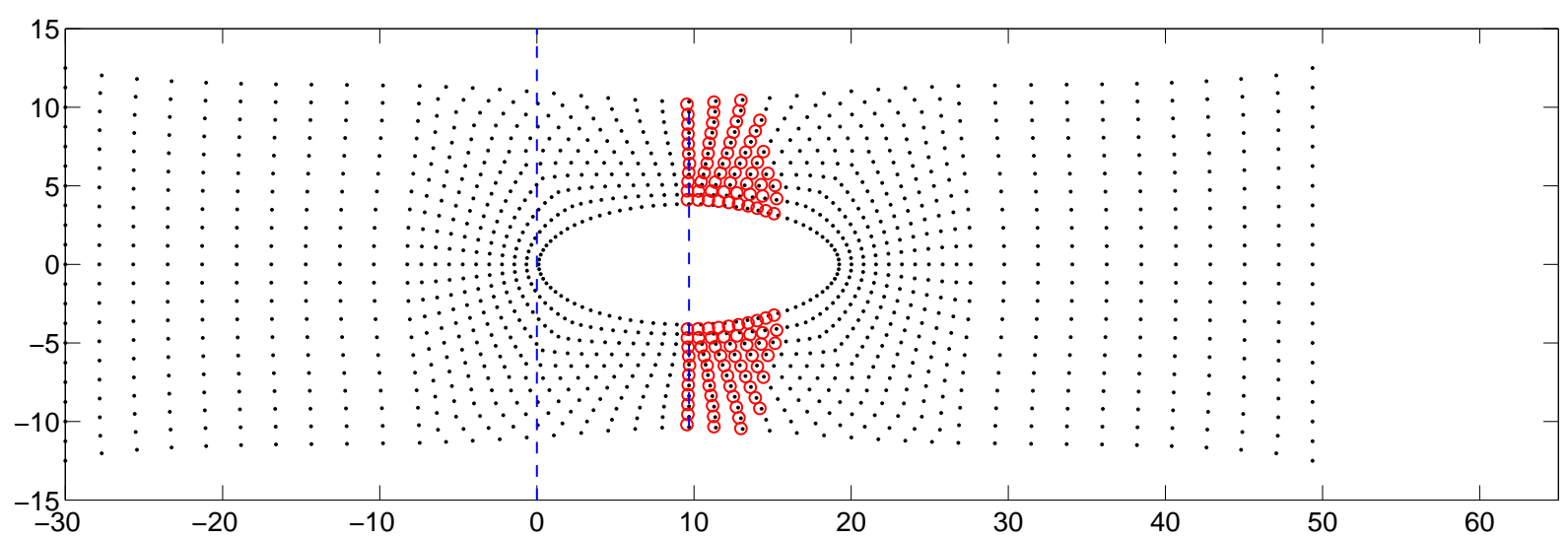

(a) 27 th time step.

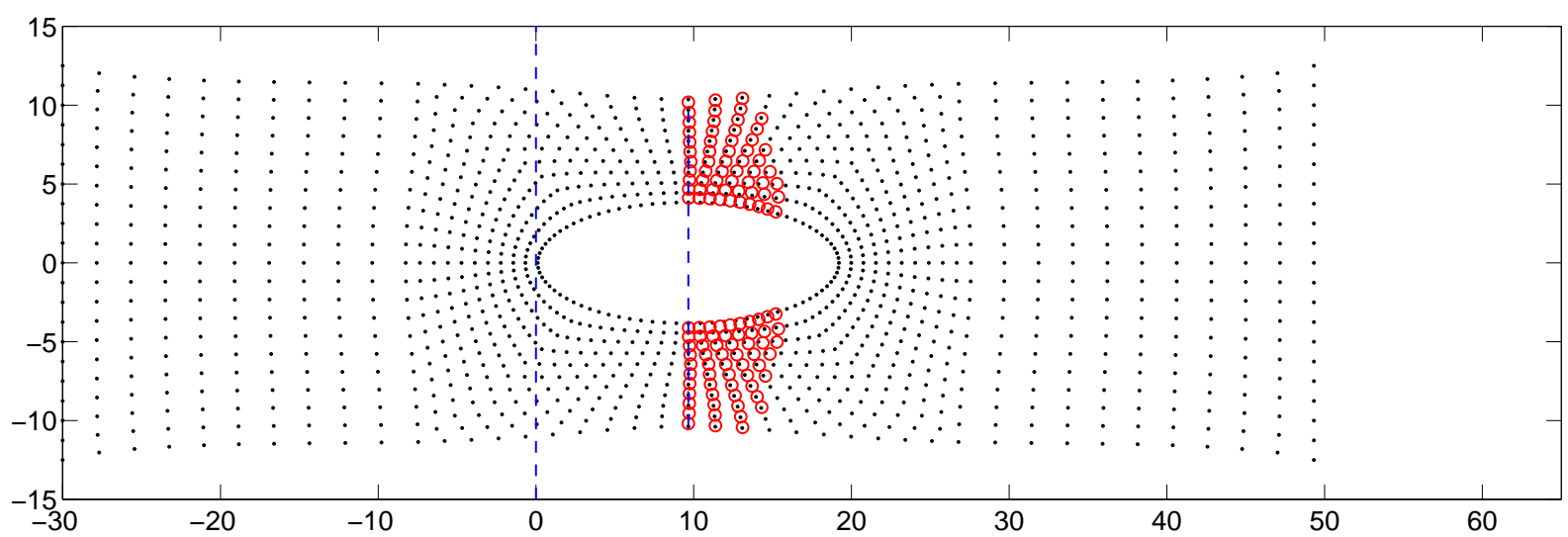

(b) 33rd time step.

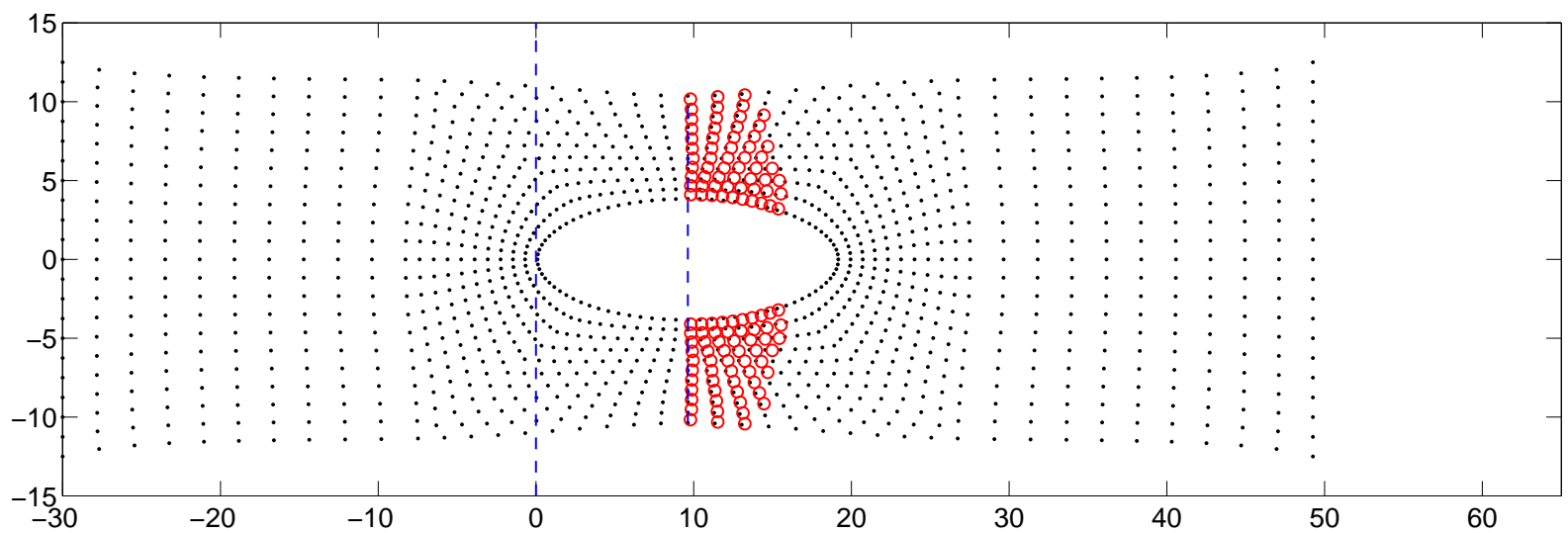

(c) 40th time step.

Figure 7.9: End of the iteration: Interpolated measured displacements (red) over the identification nodes. 
In figure 7.10 the entire FEM model with 4800 elements is shown for different time steps.

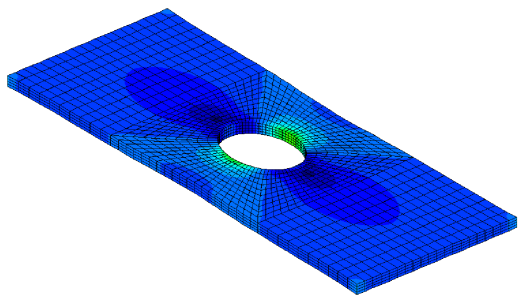

(a) 8th time step.

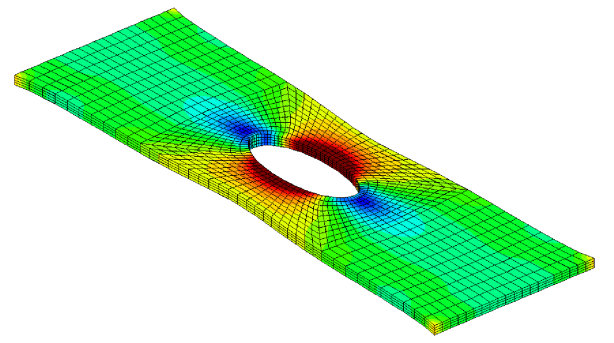

(b) 16th time step.

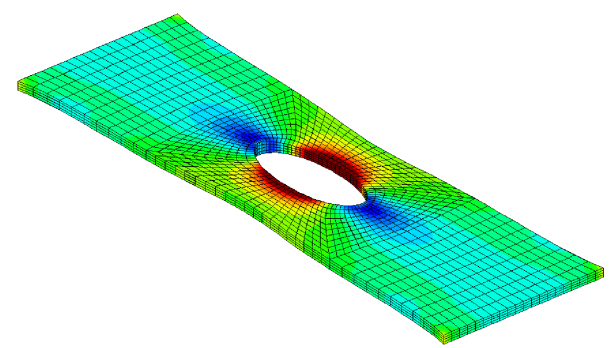

(c) 40th time step.

Figure 7.10: End of the iteration: Euclidean norm of the total Cauchy stresses. 


\subsection{Closed cell polyurethane}

In this example a parameter identification for a polyurethane foam is presented. Microcellular polyurethane elastomers exhibit a predominant closed cell structure and can be produced - with regard to the designated application area - with different densities between circa $0.35 \mathrm{~g} / \mathrm{cm}^{3}$ and $0.65 \mathrm{~g} / \mathrm{cm}^{3}$. These materials are used as, e.g. vibration damping systems, as auxiliary springs in the suspension, as damper bearings, as steel spring supports, as buffer stops, and as torque arms. Due to the cellular structure these materials display slight transverse elongation and high volume compressibility (for further reading about polyurethane chemistry and processing see, e.g. Leppkes [33], Becker et al. [6] and Klempner [29]).

A common application for polyurethane foams within automotive construction is as spring aids and bump stops (see figure 7.11). The prediction of the mechanical material behavior
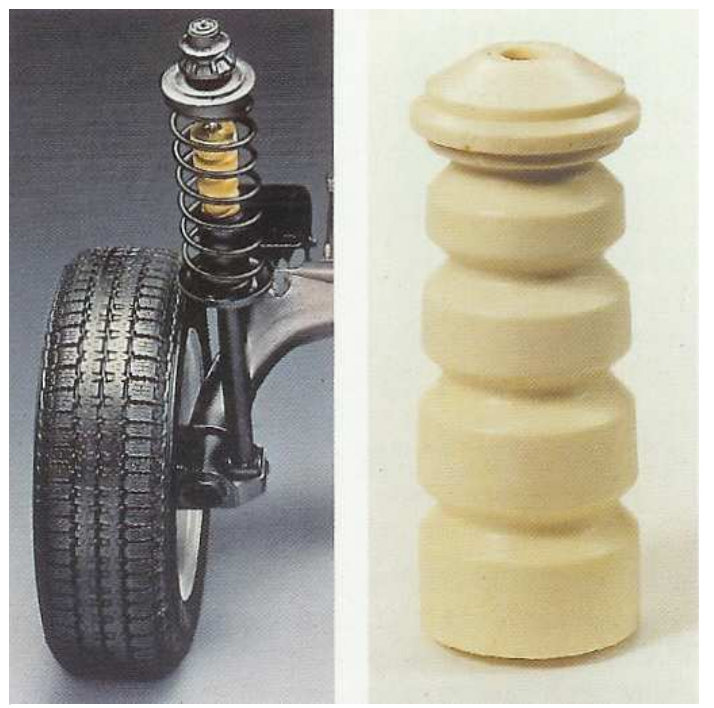

Figure 7.11: Spring aid in a spring leg (Elastogran $\mathrm{GmbH}$ ). Photo taken from R. Leppkes [33] by permission of Verlag Moderne Industrie.

of these components by means of the FE analysis becomes increasingly more important due to the increasing relevance of these parts as a contribution to the overall handling of the car. Since they are often used as secondary springs in order to control on the limit handling, especially the short time mechanical behavior is of interest for the simulation. The cellular PUR investigated in this study has been provided by VIBRACOUSTIC GMBH. Based on three different compression tests - conducted on two samples from different charges with the same density and the same chemical composition - the identification of the material parameters of the viscoelastic material law (see chapter (3)) with one relaxation module shall be estimated.

It needs to be mentioned that - due to the closed cell structure - in the first step of a compression deformation the material behavior is dominated by the deformation of the volume of the cells, and therefore is primarily dominated by the fluid part of the material. Afterwards the deformation of the solid part mainly determines the deformation characteristics. However, for the material law no particular approach for the description of the 
mechanical behavior of the cellular microstructure is made. Nevertheless the applicability of this constitutive model to the considered foam shall be investigated.

\subsubsection{Experiments}

The experimental data was determined by M. Bosseler of the 'Institute of Resource centered Product Development' under the supervision of Prof. Dr.-Ing. R. Renz, University of Kaiserslautern (see Bosseler et al. [11]).

The material is processed in straight cylinders with a diameter of $40 \mathrm{~mm}$. The specimens are $40 \mathrm{~mm}$ long, and for clamping on the ends steel round bars with the same diameter are bond (see the right photography in figure 7.12). In a compression test this geometry of the specimen in combination with its clamping will lead to inhomogeneous displacement fields.

For the measurement of the displacement field on a part of the lateral surface of the specimens the three dimensional image correlation photogrammetry (see subsection 4.2) is used. Therefore a black and white stochastic pattern is applied onto the specimen's surface. During the tests synchronized stereo images of the pattern are recorded at different load stages using CCD cameras (Vosskühler 1300, resolution 1024 x 1280 pixels) with 50mm objectives (Schneider Kreuznach). The three dimensional displacement field is computed by using a photogrammetric evaluation procedure (ARAMIS ${ }^{3}$ ).

The compression tests are conducted on a Zwick 1454 universal testing machine (load cell $\pm 20 k N)$. The load is applied in displacement control and transmitted by two parallel steel plates as shown in the left photography in figure 7.12. In order to achieve a continuous deformation measurement a smooth and bright illumination of the specimen is necessary. Therefore two fluorescent tubes from the left and the right and a small cold light spot from the front are installed.
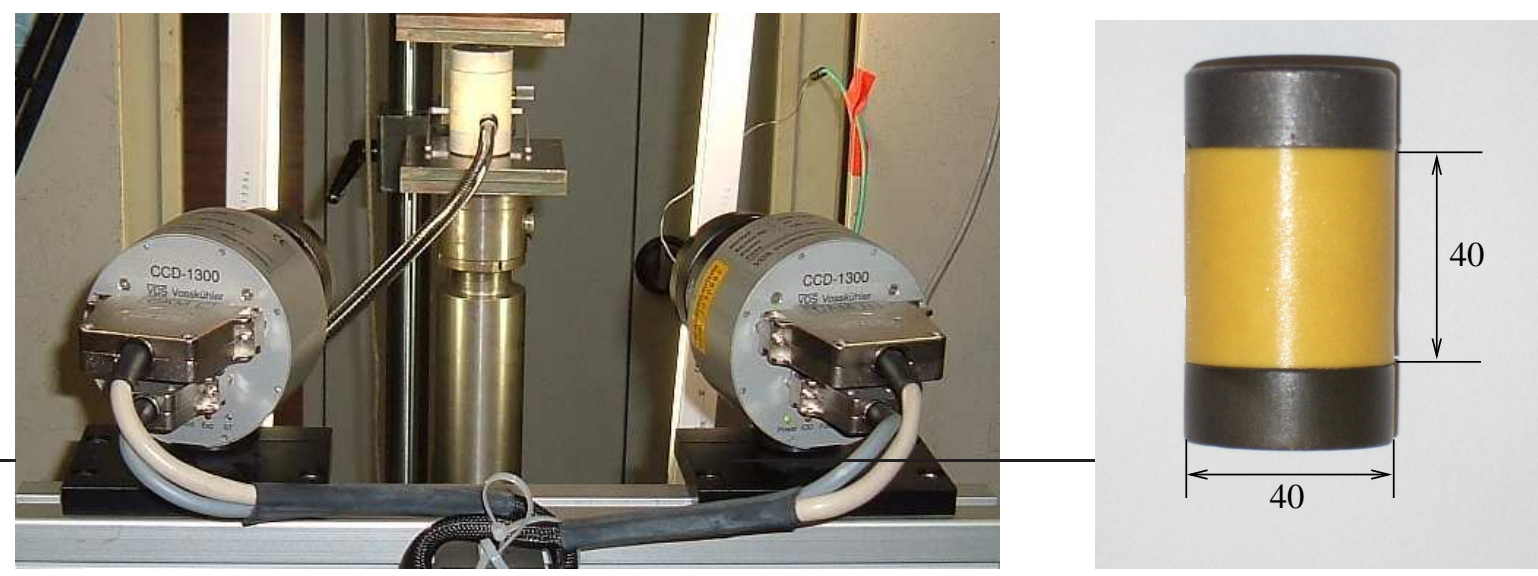

Figure 7.12: Left: CCD cameras and testing machine. Right: specimen.

Before the conduction of the test, each specimen is loaded in six load cycles with the maximal total displacement used in the respective later test. It turned out that this number of load cycles is enough to cause a saturation of damage (Mullins effect). Figure 7.13 shows the stress softening in the first four load cycles. Sufficiently long before the

\footnotetext{
${ }^{3}$ Version 5.4 (Gesellschaft für optische Messtechnik mbH)
} 
conduction of each test, the specimen is not loaded in order to exclude relaxation effects of the previous test.

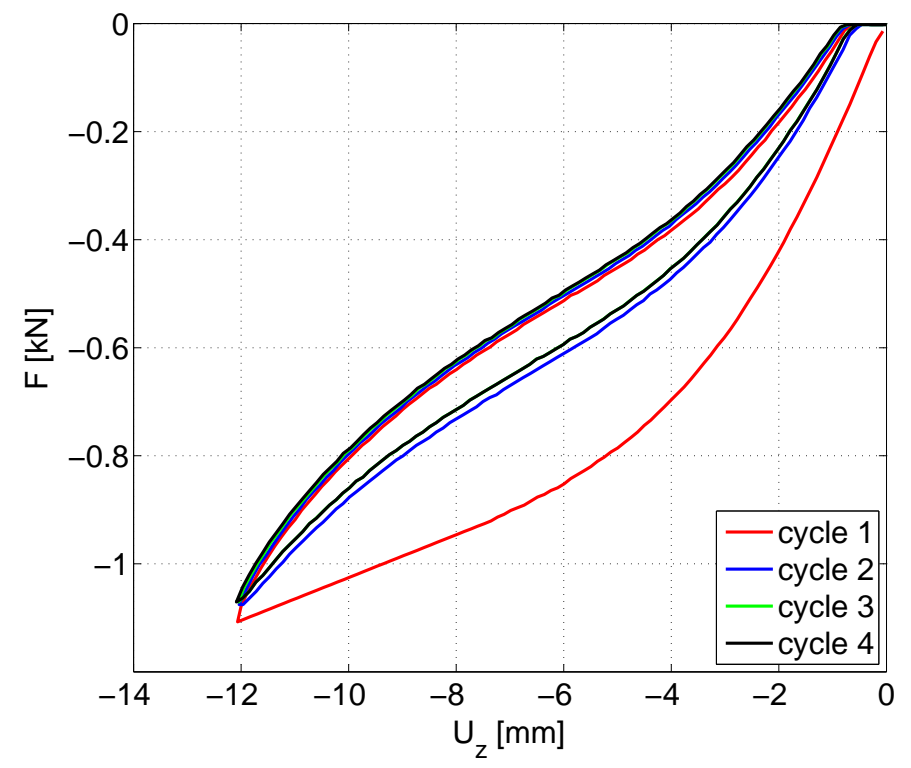

Figure 7.13: Stress softening in the first four load cycles (Mullins effect) for the cellular PUR.

The test data are the force-time curves and the three-dimensional displacement fields on the surface of the specimens. Three test programs $A, B$, and $C$ performed on two specimens are chosen.

Test $A$ is a compression test. The maximal absolute value of the displacement of $25 \mathrm{~mm}$ is applied in $75 \mathrm{~s}$. Every three seconds an image of the specimen is taken by the CCD cameras for the displacement measurement.

Test $B$ is a compression test with one load cycle. The maximal absolute value of the displacement of $11.7 \mathrm{~mm}$ is applied in $6 \mathrm{~s}$ and hold for $6 \mathrm{~s}$. Then the absolute value of the total displacement is reduced in the following $38 \mathrm{~s}$ with a rate of $-0.25 \mathrm{~mm} / \mathrm{s}$. Each $0.5 \mathrm{~s}$ an image of the specimen is taken by the CCD cameras for the displacement measurement.

Test $C$ reflects 6 loading steps with hold times up to almost full stress relaxation. The applied absolute values of the total displacements for the respective steps are $2.0 \mathrm{~mm}, 4.0 \mathrm{~mm}, 6.0 \mathrm{~mm}, 8.0 \mathrm{~mm}, 10.0 \mathrm{~mm}$ and $12.0 \mathrm{~mm}$. At the end of each loading step an image of the specimen is taken by the CCD cameras for the displacement measurement.

For the tests $B$ and $C$ the same sample is applied, whereas the sample for the test $A$ derives from another charge - nevertheless it exhibits the same density, chemical composition and pore structure. With the choice of the test programs listed above the material parameters of the viscoelastic constitutive law shall be identified for a large range of loadings within different time domains. As mentioned above, for the simulation of, e.g. spring aids and bump stops made out of the considered material, the material behavior for small loads as well as for extreme loadings is of interest. Moreover, the averaging of 
the material parameters respecting the scattering of the mechanical properties between different samples shall be included.

The corresponding force-time curves for test $A$ and test $B$ are shown in figure 7.14. In figure 7.15 the values for the measured forces after one hour of relaxation time in each of the six loading steps are marked. Note that $F$ is the total force in loading direction.

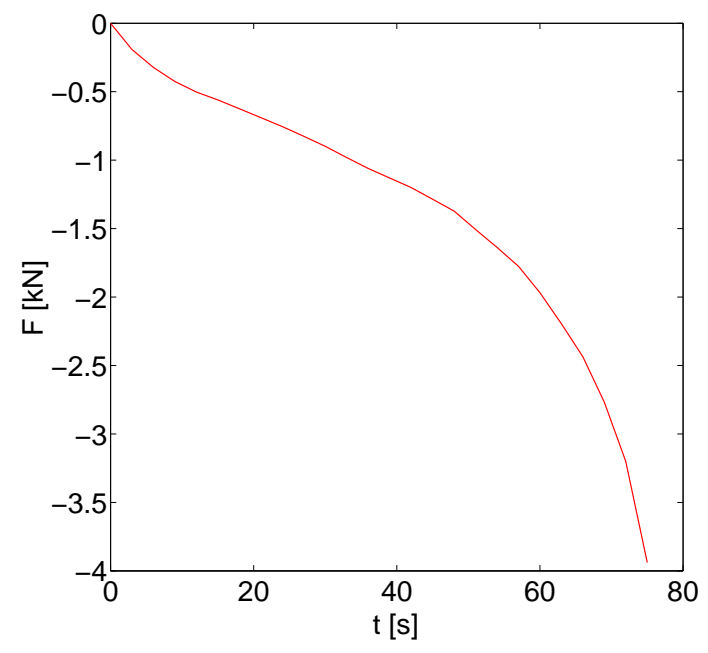

(a) Test $A$.

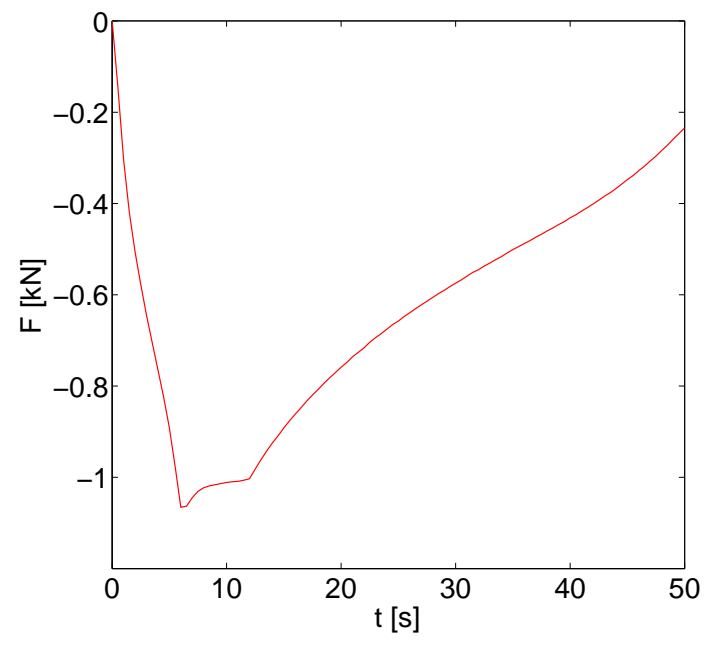

(b) Test $B$.

Figure 7.14: Measured force-time curves for the tests $A$ and $B$. These force vs. time data are used for the (force-) control of the corresponding simulations.

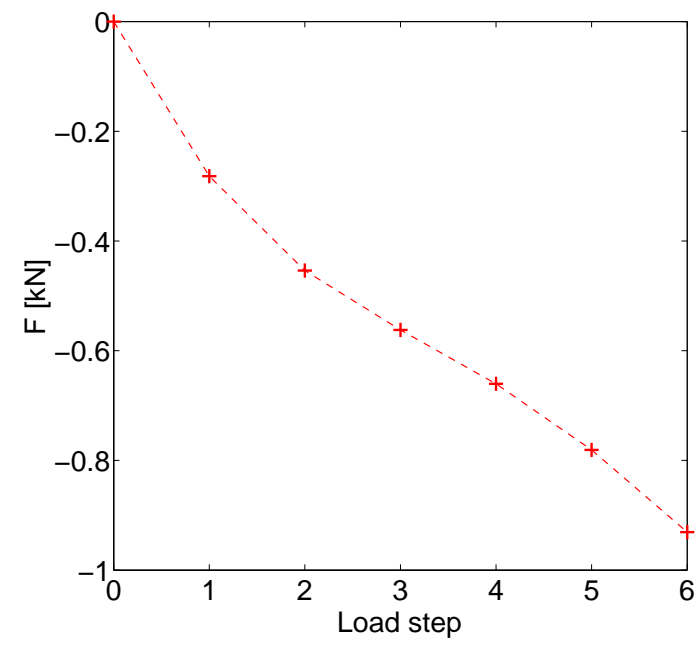

Figure 7.15: Measured force after (almost) complete relaxation in six loading levels in test $C$. These force levels are used for the (force-) control of the corresponding simulation.

\subsubsection{Interpolation of experimental data}

For the simulation of the tests two FEM discretizations of the specimen with 162 and 1296 eight-node standard displacement-based elements (Q1) are used. Thereby, symmetry 
conditions in radial direction are applied for the FE models and hence only a quarter of the base is discretized. The boundary conditions for both bases are chosen in correspondence with the experiments whereby the nodes on one base are fixed in all directions. The nodes on the other plane are fixed in the directions in the plane of the base ( $x$ and $y)$ and in longitudinal $(z)$ direction uniaxial normal forces are applied whereby warping of the plane is prevented. Rigid body motion is excluded. Figure 7.16 shows the entire FEM mesh with the discretization of the respective corresponding FEM model with 1296 elements.

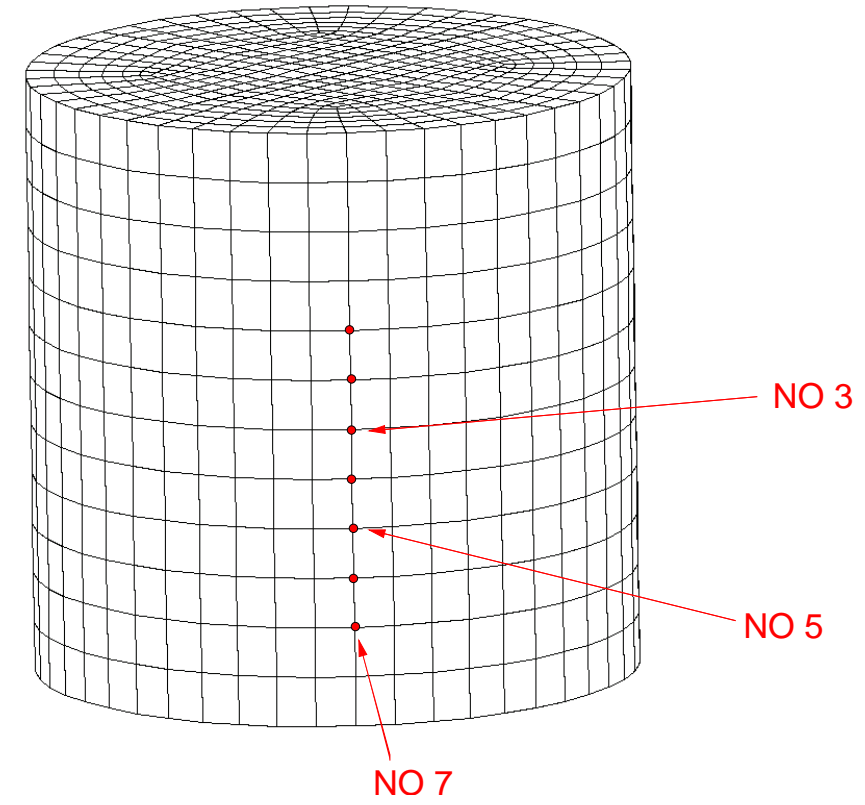

Figure 7.16: Entire FEM model with the discretization of the respective corresponding FEM model with 1296 elements; Identification nodes NO 3, NO 5 and NO 7.

In order to reduce the computational costs for the identification routine the optimal material parameters determined with the 162-element model at level one are used as initial parameters for the optimization based on the 1296-element model at level two.

Since the coordinates of the measurement points generally do not coincide with the coordinates of the identification nodes, the measured displacements are interpolated linearly on the identification nodes. For the symmetric cylinder the two dimensional displacements in longitudinal and radial direction for the measurement points on a straight line along the exterior of the specimens are interpolated on the corresponding identification nodes on the straight line along the exterior of the FE model whereby the two nearest measurement points to the respective identification node are used (see section 4.3).

In order to make the three tests $A, B$, and $C$ comparable for the later identification throughout identical identification nodes are chosen for all three tests.

\subsubsection{Least squares problem}

The identification shall be carried out concentrating on the short time viscoelastic behavior. Considering the short loading level of $6 s-$ which defines this relaxation process most 
explicitly - it becomes obvious that the material exhibits - amongst others - this very short relaxation time. Without taking into account the whole relaxation process for long time loads but instead only considering the material behavior for six loading levels with (almost) full relaxation, the identification is carried out for the viscoelastic material with one relaxation term $(N=1$ in equation (3.8)). Thereby, within the equilibrium term of the free energy function $\Psi^{\mathrm{eq}}$ - as defined in equation $(3.60)$ - the part $\left[\Psi^{\mathrm{eq}}\right]^{\mathrm{dev}}$ consists of one term, so that $\left(N_{T}=1\right)$ holds. For the one non-equilibrium part $\Psi^{k=1}$ the number of terms in $\left[\Psi^{\mathrm{k}=1}\right]^{\mathrm{dev}}$ is given by $\mathrm{N}_{\mathrm{T}}^{k=1}=1$ as well, so that the corresponding parameter set is given by $\boldsymbol{\kappa}=\left[\left[\mu_{\text {eq }}\right]_{1},\left[\alpha_{\text {eq }}\right]_{1}, \kappa_{\text {eq }},\left[\mu_{\text {neq }}^{\mathrm{k}=1}\right]_{1},\left[\alpha_{\text {neq }}^{\mathrm{k}=1}\right]_{1}, \kappa_{\text {neq }}^{\mathrm{k}=1}, \hat{\tau}^{\mathrm{k}=1}\right]^{\mathrm{T}}$.

By means of solving a combined sum of least squares for all three tests, all parameters shall be identified simultaneously. Therewith we aim at decreasing the instability problems described in section 4.5. As already mentioned, an insufficient number of testing data can cause instable or non-unique parameter estimates. If the data is incomplete it cannot cover the whole range of intended model applications (see Mahnken [40]). In addition, by simultaneous identification an optimal set of parameters in the sense of an optimal adjustment of the constitutive model - being subject to a model error itself - to all tests is achieved. As already mentioned, the consideration of all tests in one optimization iteration also leads to an improved adjustment of the imbalances which arise from scattering of experimental data.

In order to identify the parameters for the tests $A, B$, and $C$ simultaneously, the least squares approach in equation (4.8) is modified, namely

$$
\begin{aligned}
f(\boldsymbol{\kappa})= & \frac{1}{2} \sum_{i=1}^{N_{g}}[\underbrace{\sum_{j=1}^{T_{A}}\left[\boldsymbol{W}_{i j} \cdot\left[\overline{\boldsymbol{u}}_{i j}(\boldsymbol{\kappa})-\overline{\boldsymbol{u}}_{i j}^{e x p}\right]\right]^{2}}_{A}+\underbrace{\sum_{k=1}^{T_{B}}\left[\boldsymbol{W}_{i k} \cdot\left[\overline{\boldsymbol{u}}_{i k}(\boldsymbol{\kappa})-\overline{\boldsymbol{u}}_{i k}^{e x p}\right]\right]^{2}}_{B} \\
& +\underbrace{\left.\sum_{l=1}^{T_{C}}\left[\boldsymbol{W}_{i l} \cdot\left[\overline{\boldsymbol{u}}_{i l}(\boldsymbol{\kappa})-\overline{\boldsymbol{u}}_{i l}^{\exp }\right]\right]^{2}\right]}_{C},
\end{aligned}
$$

whereby $\boldsymbol{W}_{i j}, \boldsymbol{W}_{i k}$, and $\boldsymbol{W}_{i l}$ are weighting matrices (see e.g. Bard [3]) with $\boldsymbol{W}_{i j}=$ $\operatorname{diag}\left(\left[W_{i j}\right]_{1},\left[W_{i j}\right]_{2}\right), \boldsymbol{W}_{i k}=\operatorname{diag}\left(\left[W_{i k}\right]_{1},\left[W_{i k}\right]_{2}\right)$, and $\boldsymbol{W}_{i l}=\operatorname{diag}\left(\left[W_{i l}\right]_{1},\left[W_{i l}\right]_{2}\right)$. Thereby $\left[W_{i j}\right]_{1},\left[W_{i k}\right]_{1}$, and $\left[W_{i l}\right]_{1}$ are the weights for the respective displacements in longitudinal direction whereas $\left[W_{i j}\right]_{2},\left[W_{i k}\right]_{2}$, and $\left[W_{i l}\right]_{2}$ are the weights for the respective displacements in transverse (radial) direction. For the simulation of the displacement fields $\overline{\boldsymbol{u}}_{i j}(\boldsymbol{\kappa})$, $\overline{\boldsymbol{u}}_{i k}(\boldsymbol{\kappa})$ and $\overline{\boldsymbol{u}}_{i l}(\boldsymbol{\kappa})$ the FEM simulation is force-controlled based on the measured force signals shown in figures 7.14 and 7.15. Moreover, $N_{g}$ denotes the number of identification nodes which is identical for all tests. Nevertheless $N_{g}$ is dependent on the discretization for the different FE models in the identification process as described in subsection 7.3.2. The number of identification nodes at level one yields $N_{g}=N_{g}^{l e v_{1}}=4$ and at level two it yields $\left(N_{g}=N_{g}^{l e v_{2}}=7\right)$.

In test $A$ the number of time steps is $T_{A}=25$ with a time step length of $\Delta t=3 \mathrm{~s}$. For $B$ the number of time steps corresponds to $T_{B}=100$ with a time step length of $\Delta t=0.5 \mathrm{~s}$. Within test $C$ we account for a total number of 6 time steps with a time step length of $\Delta t \rightarrow \infty$, which means that only the equilibrium terms are contributing to the simulation results for these steps. The displacements calculated by FEM $\overline{\boldsymbol{u}}_{i j}(\boldsymbol{\kappa}), \overline{\boldsymbol{u}}_{i k}(\boldsymbol{\kappa}), \overline{\boldsymbol{u}}_{i l}(\boldsymbol{\kappa})$ 
and the measured displacements $\overline{\boldsymbol{u}}_{i j}^{\text {exp }}, \overline{\boldsymbol{u}}_{i k}^{\text {exp }}, \overline{\boldsymbol{u}}_{i l}^{\text {exp }}$ have, respectively, two displacement directions. Thus for the FEM discretization with 1296 elements the number of terms in the least square sum adds up to $7 \times[25+100+6] \times 2=1834$ per iteration step of the optimization algorithm.

The longitudinal displacements are larger compared with the transverse (radial) displacements. In addition, we want to emphasize some particular time steps of the tests as well as the tests relative to each other. Accordingly, the matrices $\boldsymbol{W}_{i j}, \boldsymbol{W}_{i k}$, and $\boldsymbol{W}_{i l}$ referring to each time step of the tests are incorporated.

For all time steps $1 \leq j \leq T_{A}$ and all identification nodes $1 \leq i \leq N_{g}$ within test $A$ we assume

$$
\boldsymbol{W}_{i j}=\operatorname{diag}(4,6) \text {. }
$$

Moreover, the weights for test $B$ are

$$
\boldsymbol{W}_{i k}=\operatorname{diag}(1,8) \quad \forall 1 \leq i \leq N_{g} \wedge 1 \leq j \leq T_{B}
$$

and for test $\mathrm{C}$ we apply

$$
\boldsymbol{W}_{i l}=\operatorname{diag}(1,4) \quad \forall 1 \leq i \leq N_{g} \wedge 1 \leq k \leq T_{C}
$$

Note that different weights will lead to a different solution.

Due to the non-convexity of the objective function and the existence of (many) local minima the hybrid method of application of the Levenberg-Marquard method to find different local minima for stochastically chosen start parameter sets is used (see section 4.4). Basically it can be observed that for each new starting point a number of eight iteration steps are enough to detect if the starting point leads to a local minimum or whether this iteration can be discarded because of divergence. In order to save computing time the hybrid method described above is mainly applied in the first level, whereby the FEM model with 162 elements is used for the identification routine.

\subsubsection{Identified parameter sets}

\section{Identified material parameters}

First the first level is considered, whereby the FEM model with 162 elements is used for the identification routine. Out of all investigated local minima the two parameter sets $\boldsymbol{\kappa}=\boldsymbol{\kappa}_{l m_{1}}^{l e v_{1}}$ and $\boldsymbol{\kappa}=\boldsymbol{\kappa}_{l m_{2}}^{l e v_{1}}$ are generating the smallest weighted sums of squared differences, whereby these sums are almost equal (see table 7.4). For the identification at level two

Table 7.4: Determined local minima at level one.

\begin{tabular}{|l|l|l|l|l|l|l|l|}
\hline & $\begin{array}{l}{\left[\mu_{\text {eq }}\right]_{1}} \\
{[\mathrm{MPa}]}\end{array}$ & $\begin{array}{l}{\left[\alpha_{\text {eq }}\right]_{1}} \\
{[-]}\end{array}$ & $\begin{array}{l}\kappa_{\text {eq }} \\
{[\mathrm{MPa}]}\end{array}$ & $\begin{array}{l}{\left[\mu_{\text {neq }}^{\mathrm{k}=1}\right]_{1}} \\
{[\mathrm{MPa}]}\end{array}$ & $\begin{array}{l}{\left[\alpha_{\text {neq }}^{\mathrm{k}=1}\right]_{1}} \\
{[-]}\end{array}$ & $\begin{array}{l}\kappa_{\text {neq }}^{\mathrm{k}=1} \\
{[\mathrm{MPa}]}\end{array}$ & $\begin{array}{l}\hat{\tau}^{\mathrm{k}=1} \\
{[\mathrm{~s}]}\end{array}$ \\
\hline \hline$\kappa_{l m_{1}}^{\text {lev }_{1}}$ & 0.80232 & 1.69847 & 1.19092 & 0.62331 & 19.83460 & 9.63142 & 0.14589 \\
\hline$\kappa_{l m_{2}}^{\text {lev }_{1}}$ & 0.76338 & 1.75274 & 1.22298 & 0.71792 & 10.04860 & 10.17334 & 0.21500 \\
\hline
\end{tabular}


these parameter sets are chosen as start parameters. The iterations at the second level with 1296 elements yield only little decrease of the sum of squared differences and also do not change the respective parameter sets significantly as it can be seen in table 7.5. In the following the parameter set $\boldsymbol{\kappa}_{l m_{2}}^{\text {lev }}$ will be denominated as 'optimal' because its

Table 7.5: Determined local minima at level two.

\begin{tabular}{|l|l|l|l|l|l|l|l|}
\hline & $\begin{array}{l}{\left[\mu_{\mathrm{eq}}\right]_{1}} \\
{[\mathrm{MPa}]}\end{array}$ & $\begin{array}{l}{\left[\alpha_{\mathrm{eq}}\right]_{1}} \\
{[-]}\end{array}$ & $\begin{array}{l}\kappa_{\text {eq }} \\
{[\mathrm{MPa}]}\end{array}$ & $\begin{array}{l}{\left[\mu_{\text {neq }}^{\mathrm{k}=1}\right]_{1}} \\
{[\mathrm{MPa}]}\end{array}$ & $\begin{array}{l}{\left[\alpha_{\text {neq }}^{\mathrm{k}=1}\right]_{1}} \\
{[-]}\end{array}$ & $\begin{array}{l}\kappa_{\text {neq }}^{\mathrm{k}=1} \\
{[\mathrm{MPa}]}\end{array}$ & $\begin{array}{l}\hat{\tau}^{\mathrm{k}=1} \\
{[\mathrm{~s}]}\end{array}$ \\
\hline \hline $\boldsymbol{\kappa}_{l m_{1}}^{\text {lev }}$ & 0.78486 & 1.73661 & 1.17537 & 0.61717 & 19.83313 & 9.62710 & 0.14547 \\
\hline $\boldsymbol{\kappa}_{l m_{2}}^{l e v_{2}}$ & 0.77276 & 1.77816 & 1.16421 & 0.72819 & 10.04931 & 10.17078 & 0.21958 \\
\hline
\end{tabular}

corresponding verification shows that the simulation of the tests with these parameters yields a better time dependent behavior - especially in test $B$ - than with the parameters $\kappa_{l m_{1}}^{l e v_{2}}$

Remark 7.2.1 Since the considered material is mainly used for components which are loaded under compression within their application areas, only compression tests are considered. Hence, the tests have too few information about the (viscoelastic) behavior under tension. The validity of the identified set of parameters for tension-dominated loading, however, is not investigated in this work.

Remark 7.2.2 Note that the identified compression modulus for the equilibrium term $\kappa_{\mathrm{eq}}$ is - in combination with the parameters for the deviatoric part of the equilibrium energy and in comparison to the respective parameters for compact elastomers - small, which underlines the well known fact that these kinds of cellular materials display only slight transverse strain and high volume compressibilities.

\section{Instabilities in the solution}

As mentioned above, due to instabilities there exist several parameter sets which lead to a insignificantly higher least squares sum, which is - as discussed in section 4.5 - a common problem in parameter estimation. In order to evaluate the significance for the quality of the solution, the correlation matrix for the optimal parameters shown in table 7.6 (see also equations (4.15) and (4.16)) is considered. There are two categories of parameters

Table 7.6: Correlation matrix for the set of optimal parameters at level two.

\begin{tabular}{|c||l|l|l|l|l|l|l|}
\hline & {$\left[\mu_{\mathrm{eq}}\right]_{1}$} & {$\left[\alpha_{\mathrm{eq}}\right]_{1}$} & $\kappa_{\mathrm{eq}}$ & {$\left[\mu_{\text {neq }}^{\mathrm{k}=1}\right]_{1}$} & {$\left[\alpha_{\text {neq }}^{\mathrm{k}=1}\right]_{1}$} & $\kappa_{\text {neq }}^{\mathrm{k}=1}$ & $\hat{\tau}^{\mathrm{k}=1}$ \\
\hline \hline$\left[\mu_{\mathrm{eq}}\right]_{1}$ & 1.000 & -1.000 & -0.027 & 0.310 & -0.305 & -0.164 & 0.172 \\
\hline$\left[\alpha_{\mathrm{eq}}\right]_{1}$ & -1.000 & 1.000 & 0.021 & -0.313 & 0.308 & 0.169 & -0.177 \\
\hline$\kappa_{\mathrm{eq}}$ & -0.027 & 0.021 & 1.000 & -0.018 & 0.020 & -0.006 & -0.055 \\
\hline$\left[\mu_{\text {neq }}^{\mathrm{k}=1}\right]_{1}$ & 0.310 & -0.313 & -0.018 & 1.000 & -1.000 & -0.896 & 0.897 \\
\hline$\left[\alpha_{\text {neq }}^{\mathrm{k}=1}\right]_{1}$ & -0.305 & 0.308 & 0.020 & -1.000 & 1.000 & 0.904 & -0.905 \\
\hline$\kappa_{\text {neq }}^{\mathrm{k}=1}$ & -0.164 & 0.169 & -0.006 & -0.896 & 0.904 & 1.000 & -0.995 \\
\hline$\hat{\tau}^{\mathrm{k}=1}$ & 0.172 & -0.177 & -0.055 & 0.897 & -0.905 & -0.995 & 1.000 \\
\hline
\end{tabular}


which correlate.

Firstly, the pairs of parameters $\left(\left[\mu_{\mathrm{eq}}\right]_{1},\left[\alpha_{\mathrm{eq}}\right]_{1}\right)$ and $\left(\left[\mu_{\mathrm{neq}}^{\mathrm{k}=1}\right]_{1},\left[\alpha_{\mathrm{neq}}^{\mathrm{k}=1}\right]_{1}\right)$ have correlation numbers equal to \pm 1 . This is due to the structure of the Ogden-model which can lead to overparametrization even for only one deviatoric term within the corresponding part of the energy. Since only parameters within the particular deviatoric terms are affected, these correlations - though the respective parameters are instable in relation to each other will not have a significant influence on the quality of the solution.

Secondly, all parameters for the relaxation module have very high correlation numbers in relation to each other. Generally - as mentioned in subsection 7.1.3 - instabilities in finding one fixed relaxation time are due to the complex viscoelastic behavior of elastomers. On the one hand, within the constitutive law there is no explicit approach for the description of the cellular structure, and a constitutive law cannot perfectly describe all characteristics of a material anyhow. In addition these parameters have to fit to the viscoelastic behavior in one load cycle (test $B$ ) and a monotonic compression test (test $B$ ) with different load rates at the same time. On the other hand, experimental data is always subject to errors (measurement errors, scattering), especially for time-dependent material behavior, which makes it rather difficult to determine one fixed relaxation time $\hat{\tau}^{\mathrm{k}=1}$ for multiple affected tests. Nevertheless it needs to be mentioned, that these correlation numbers are so high, that the parameters for the whole viscous part of the constitutive model can be specified as being indifferent. The following verification in which the results of the simulation are compared to the experimental measurements gives conclusion about the significance of these instabilities for the quality of the identified parameters.

Remark 7.2.3 Note that the correlation coefficients of the equilibrium compression modulus $\kappa_{\mathrm{eq}}$ between all the other parameters are very small which is an indicator for the good stability of $\kappa_{\mathrm{eq}}$ at the solution. Moreover, this is an important result for the evaluation of the reliability of the identified parameters since the considered material is under compression in the tests - as well as in the mechanical application area as, e.g. a bump stop.

\subsubsection{Verification}

Within the verification procedure the simulated data - calculated as based on the optimal parameters $\boldsymbol{\kappa}_{l m_{2}}^{l e v_{2}}$ for the FEM model with 1296 elements - is compared with the experimental data used for the parameter identification. By this the quality of the identified parameters and also the general ability of the constitutive model to describe the viscoelastic behavior of the material is analyzed. In particular, three representative identification nodes out of $N_{g}=7$ are chosen which are referred to by numbers 3,5 and 7 as shown in figure 7.16. Note that $U_{z}$ is the displacement in longitudinal (axial) direction and $U_{r}$ is the displacement in transverse (radial) direction. 


\section{Verification for test A}
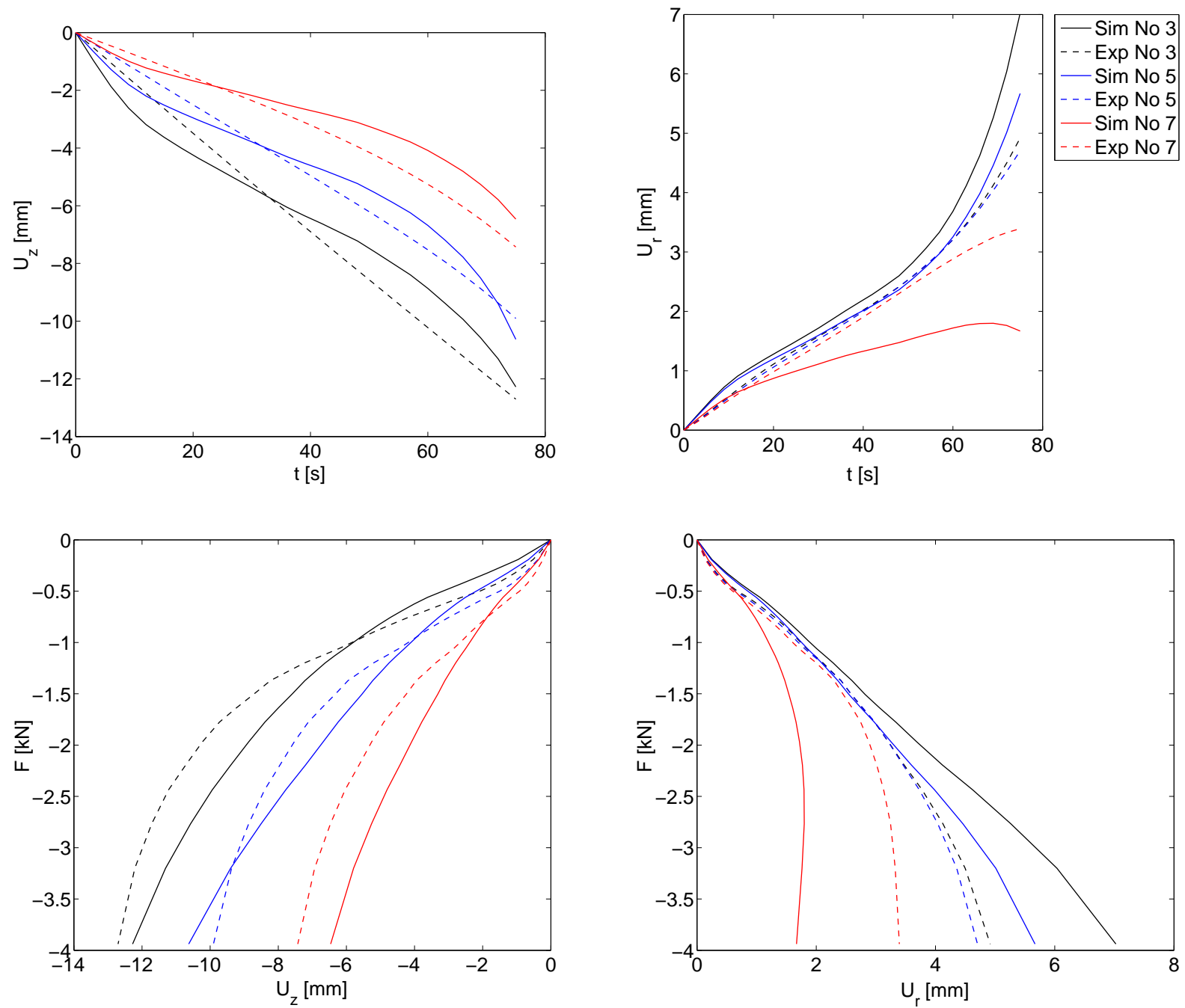

Figure 7.17: End of the identification iteration for test $A$. Displacement versus time curves and force versus displacement curves for three representative identification nodes for the experimental and simulated data. 


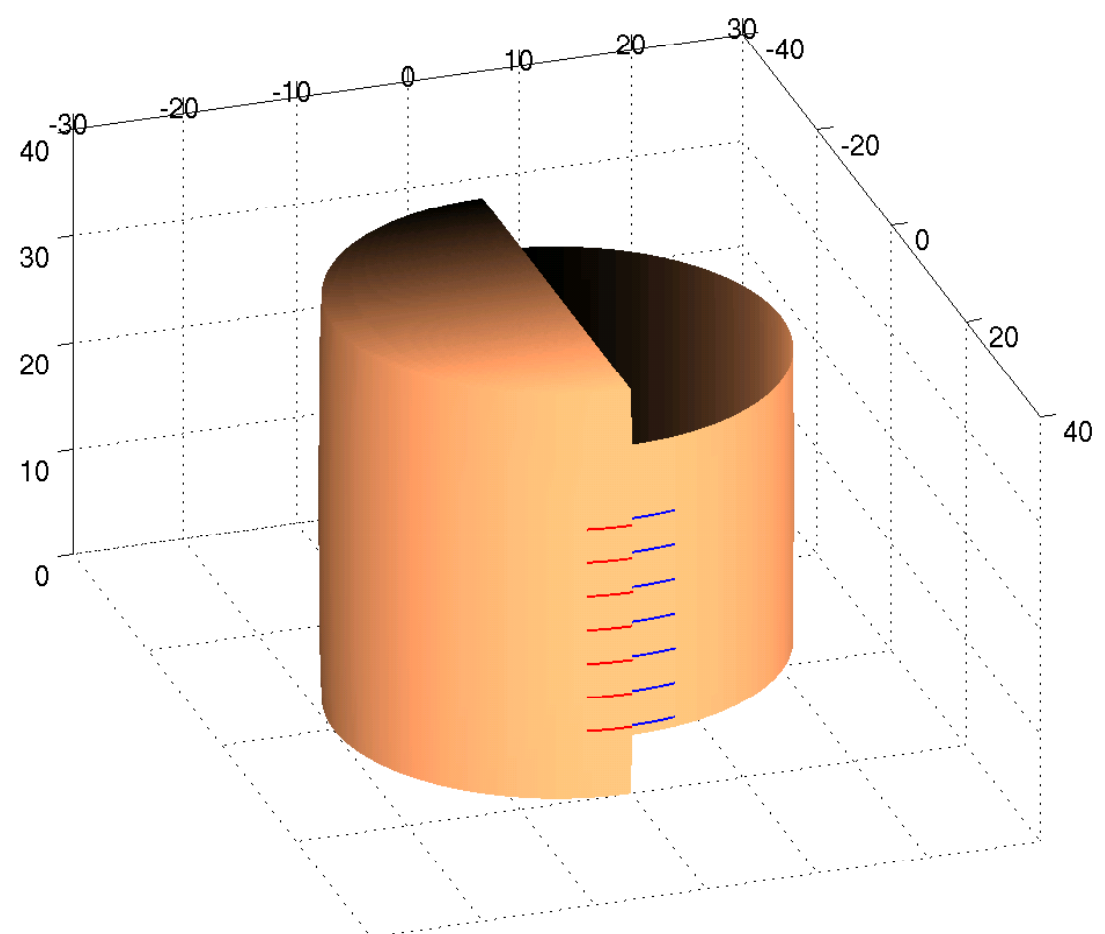

(a) 1th time step.

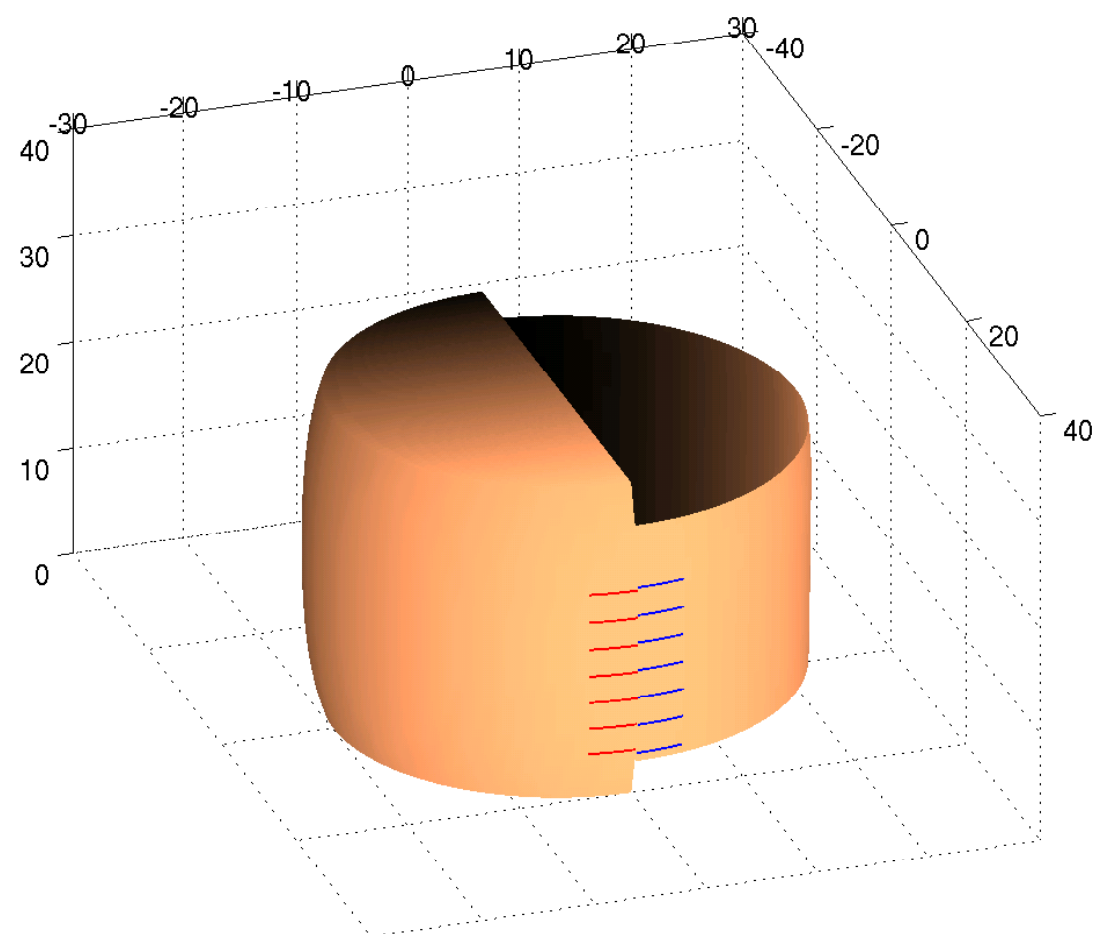

(b) 10th time step.

Figure 7.18: End of the iteration for test $A$ : Left sides of the lateral surface areas: Simulation of the specimen with red marked identification nodes; Right sides of the lateral surface areas: Measured displacement field in the experiment (only a part of the specimen is measured) with blue marked interpolated measured displacements. 


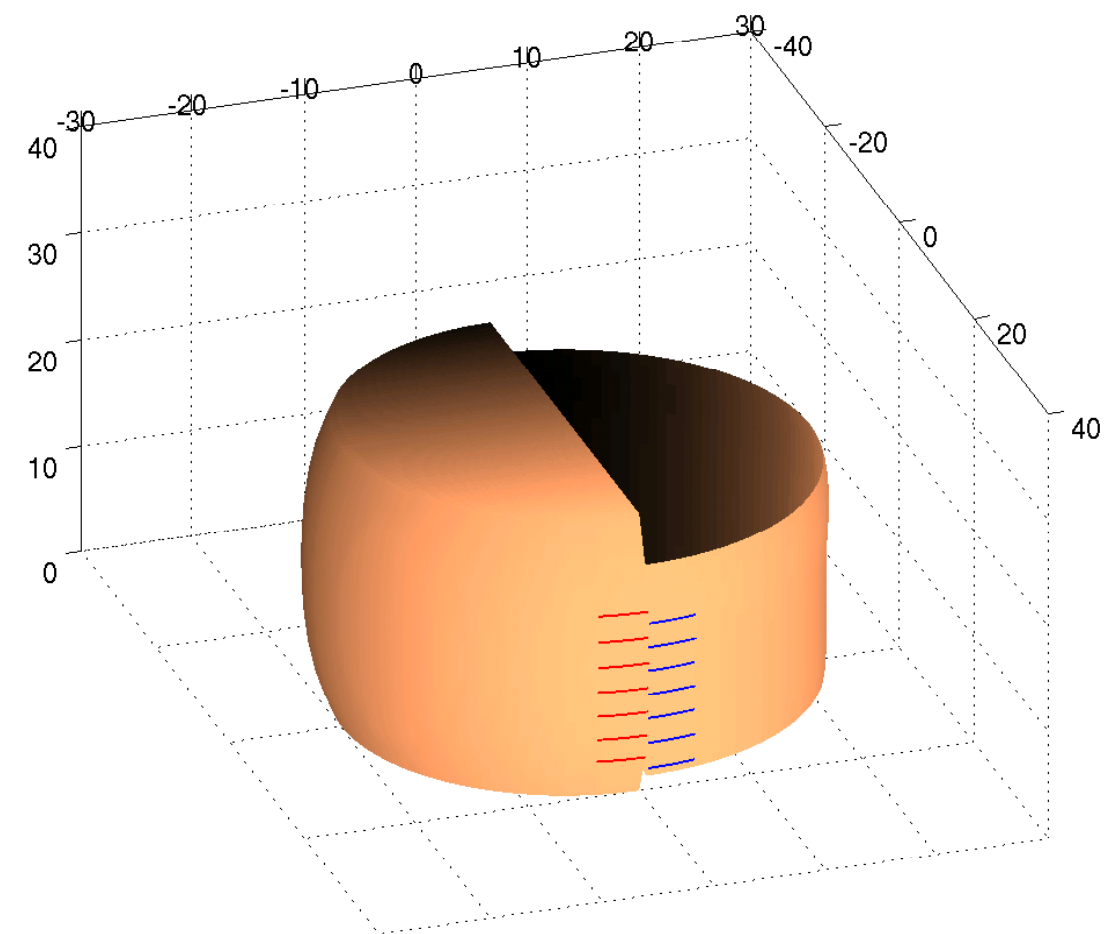

(a) 15th time step.

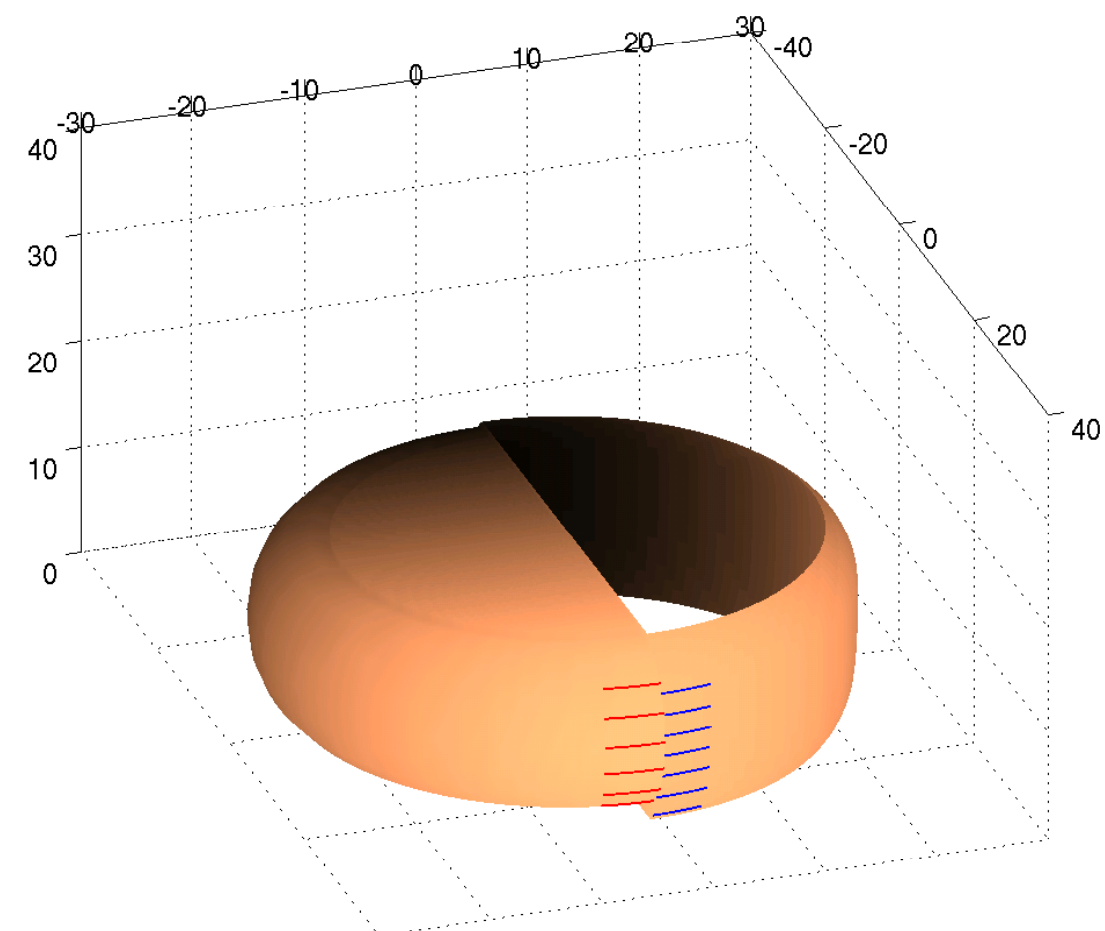

(b) 25th time step.

Figure 7.19: End of the iteration for test $A$ : Left sides of the lateral surface areas: Simulation of the specimen with red marked identification nodes; Right sides of the lateral surface areas: Measured displacement field in the experiment (only a part of the specimen is measured) with blue marked interpolated measured displacements. 


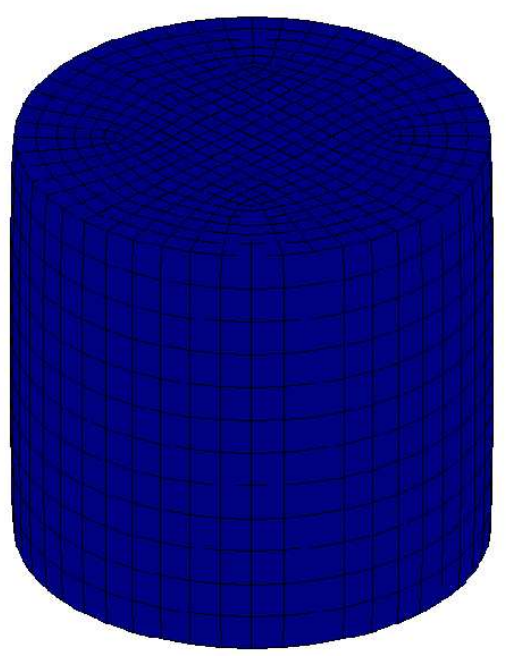

(a) 1th time step.

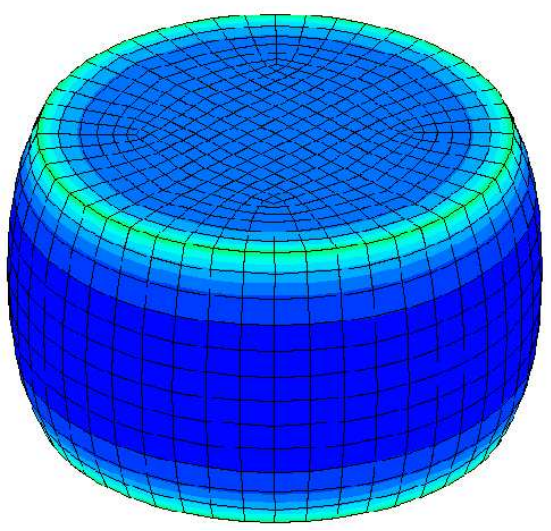

(c) 15 th time step.

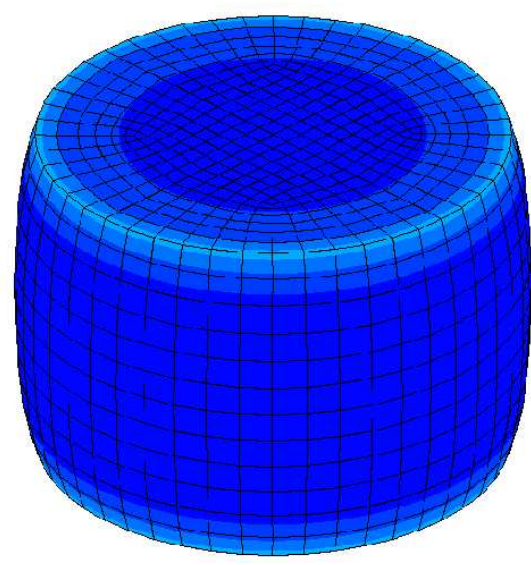

(b) 10th time step.

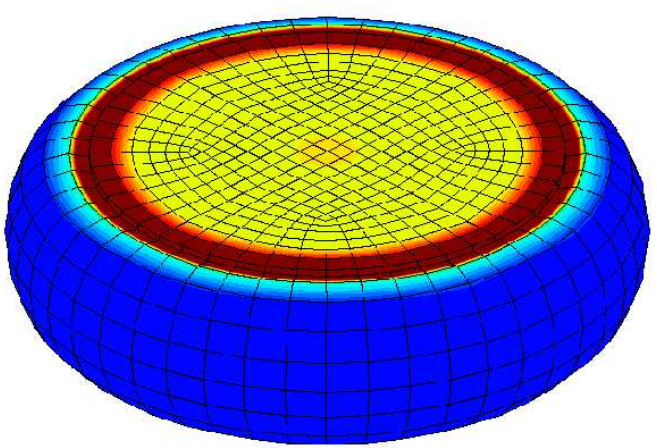

(d) 25th time step.

Figure 7.20: End of the iteration for test $A$ : Euclidean norm of the total Cauchy stresses. 


\section{Verification for test $B$}
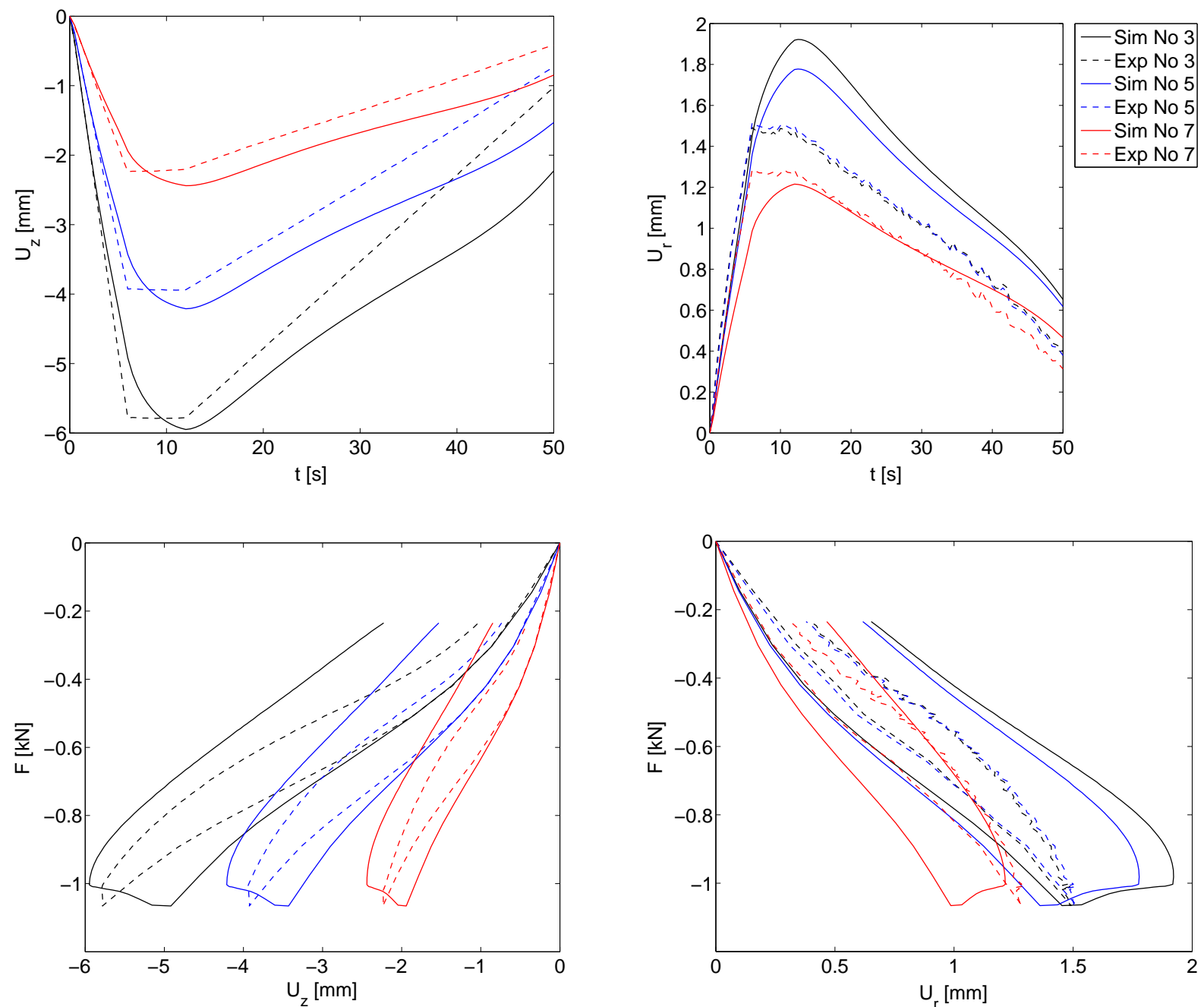

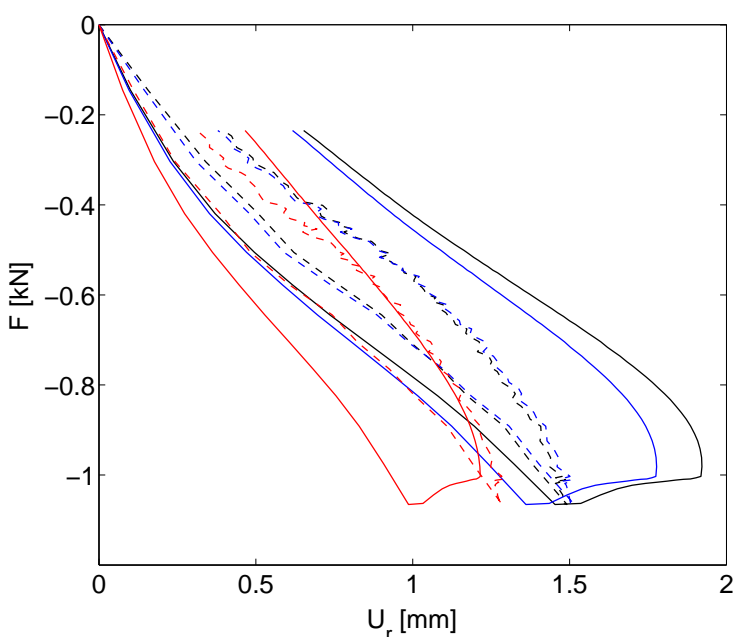

Figure 7.21: End of the identification iteration for test $B$. Displacement versus time curves and force versus displacement curves for three representative identification nodes for the experimental and simulated data. 


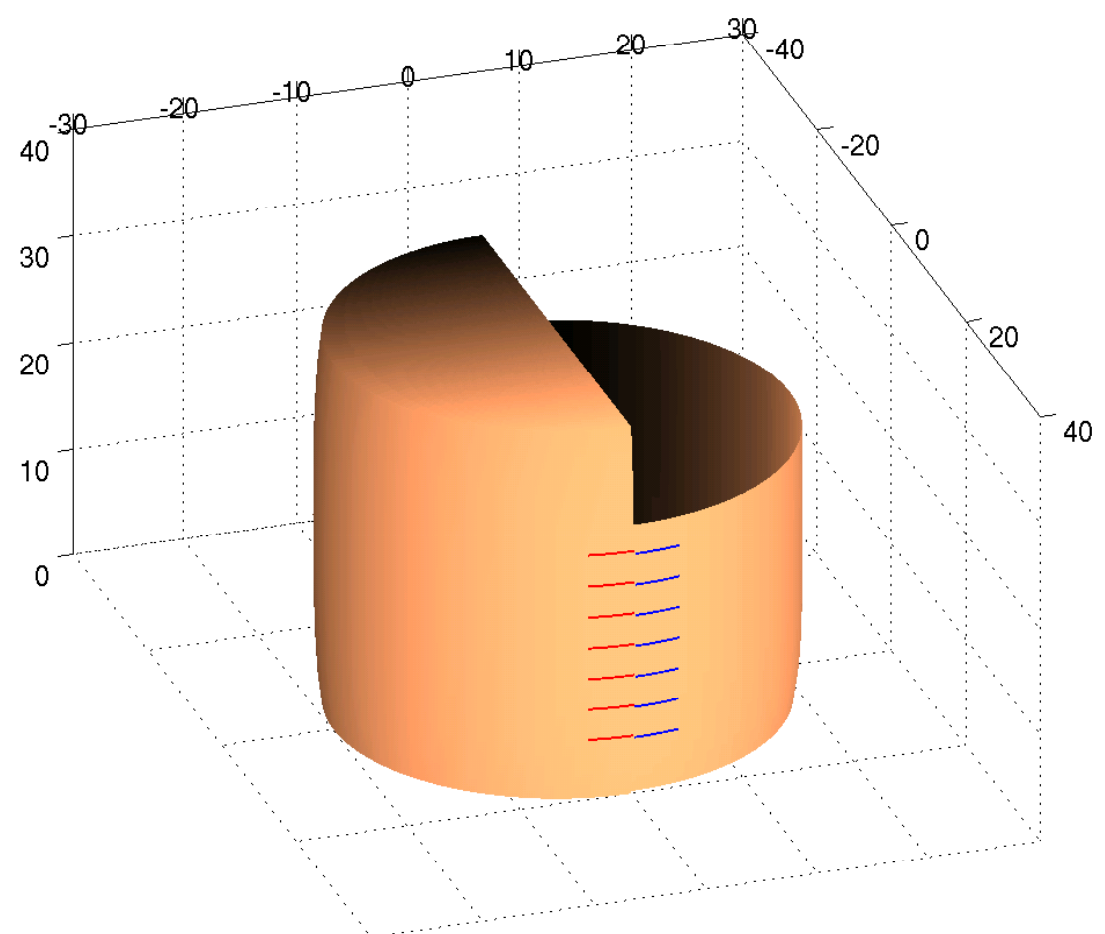

(a) 6th time step.

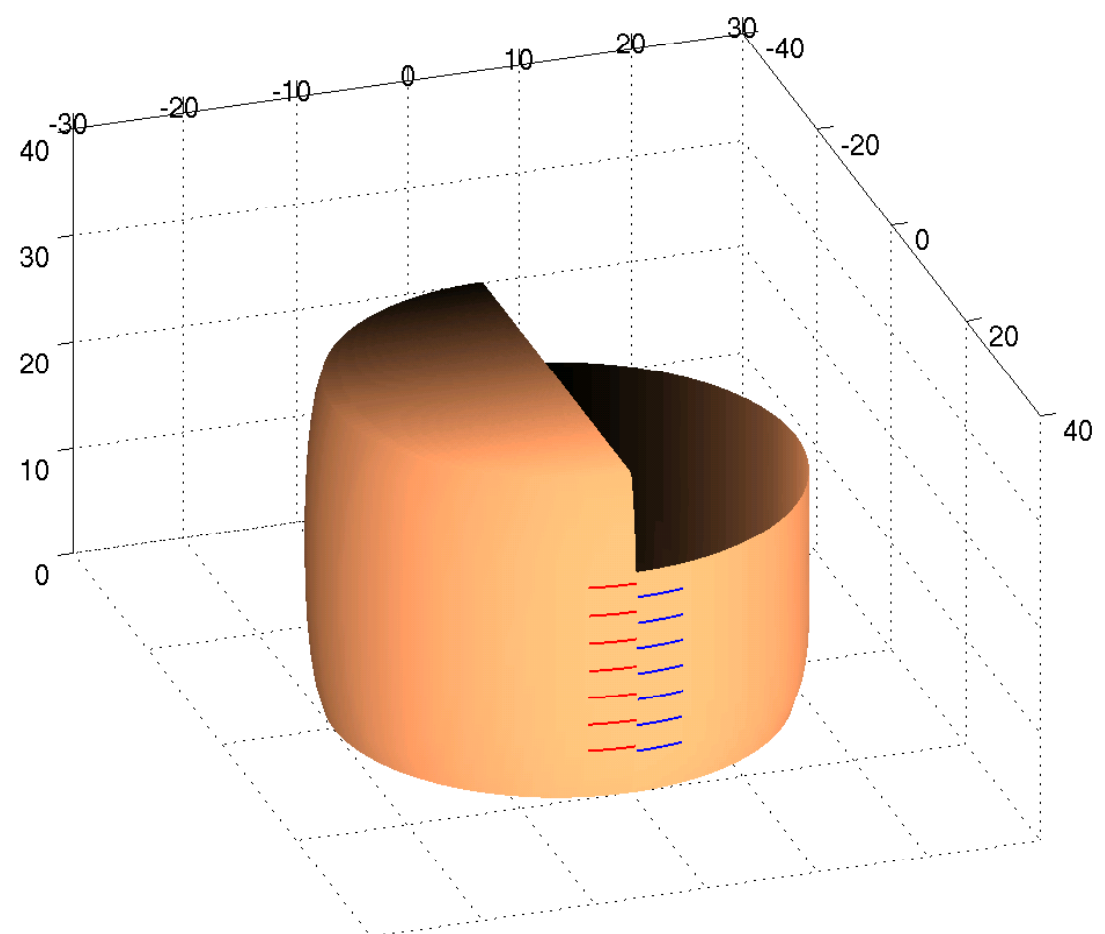

(b) 12 th time step.

Figure 7.22: End of the iteration for test $B$ : Left sides of the lateral surface areas: Simulation of the specimen with red marked identification nodes; Right sides of the lateral surface areas: Measured displacement field in the experiment (only a part of the specimen is measured) with blue marked interpolated measured displacements. 


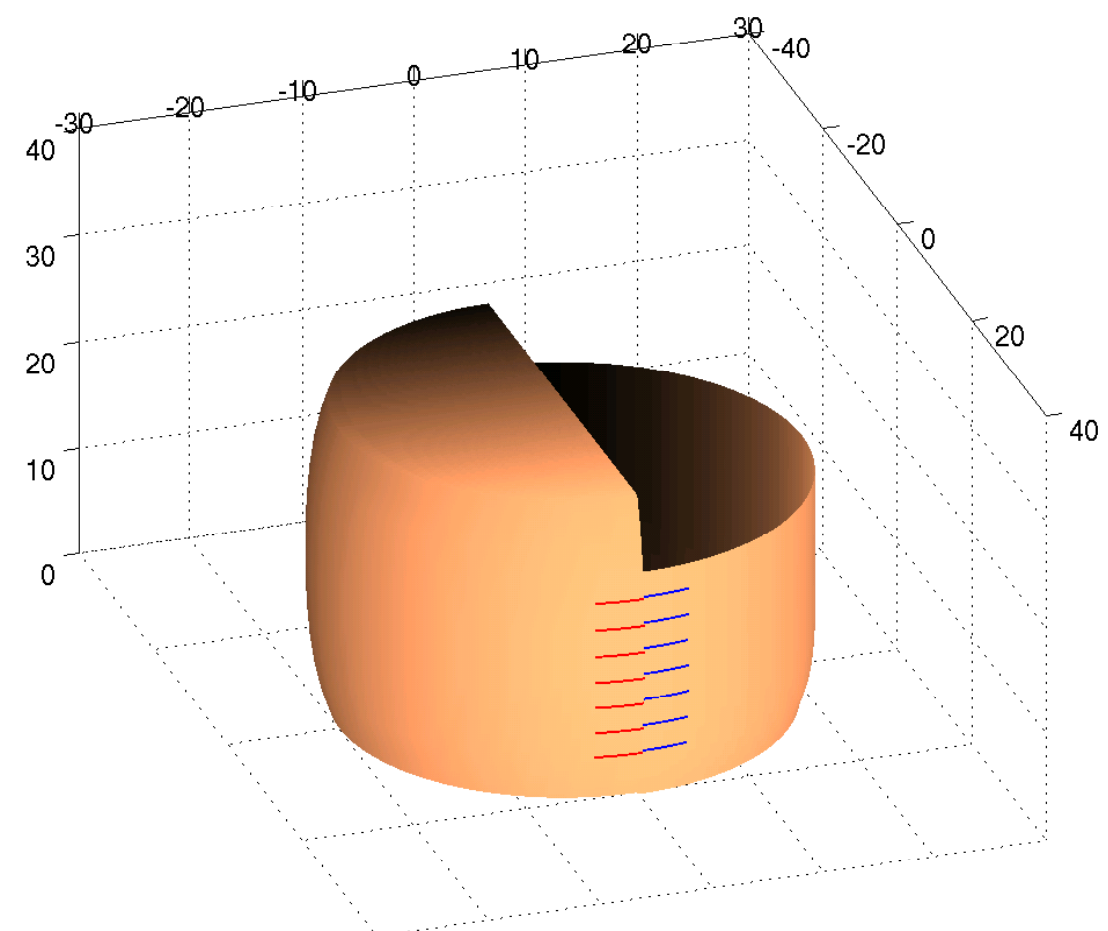

(a) 24th time step.

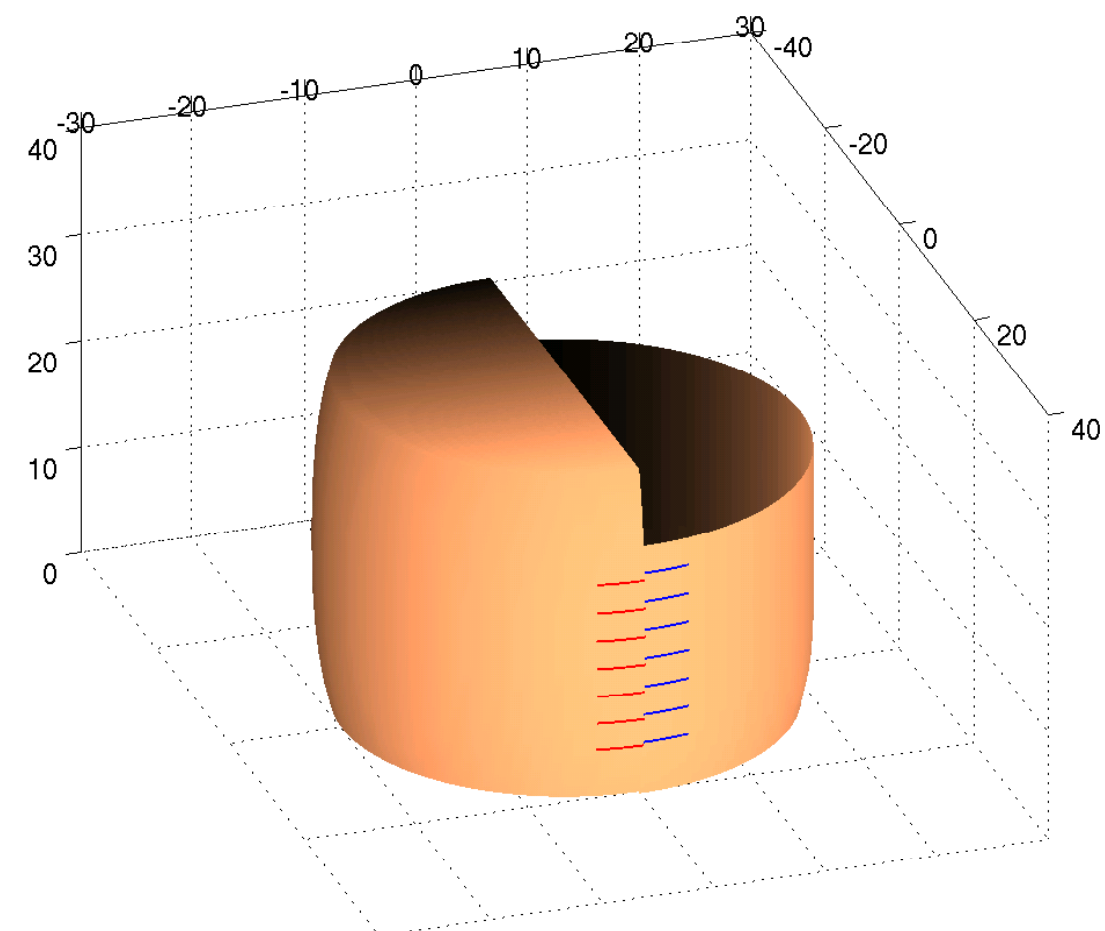

(b) 48th time step.

Figure 7.23: End of the iteration for test $B$ : Left sides of the lateral surface areas: Simulation of the specimen with red marked identification nodes; Right sides of the lateral surface areas: Measured displacement field in the experiment (only a part of the specimen is measured) with blue marked interpolated measured displacements. 


\section{Verification for test C}
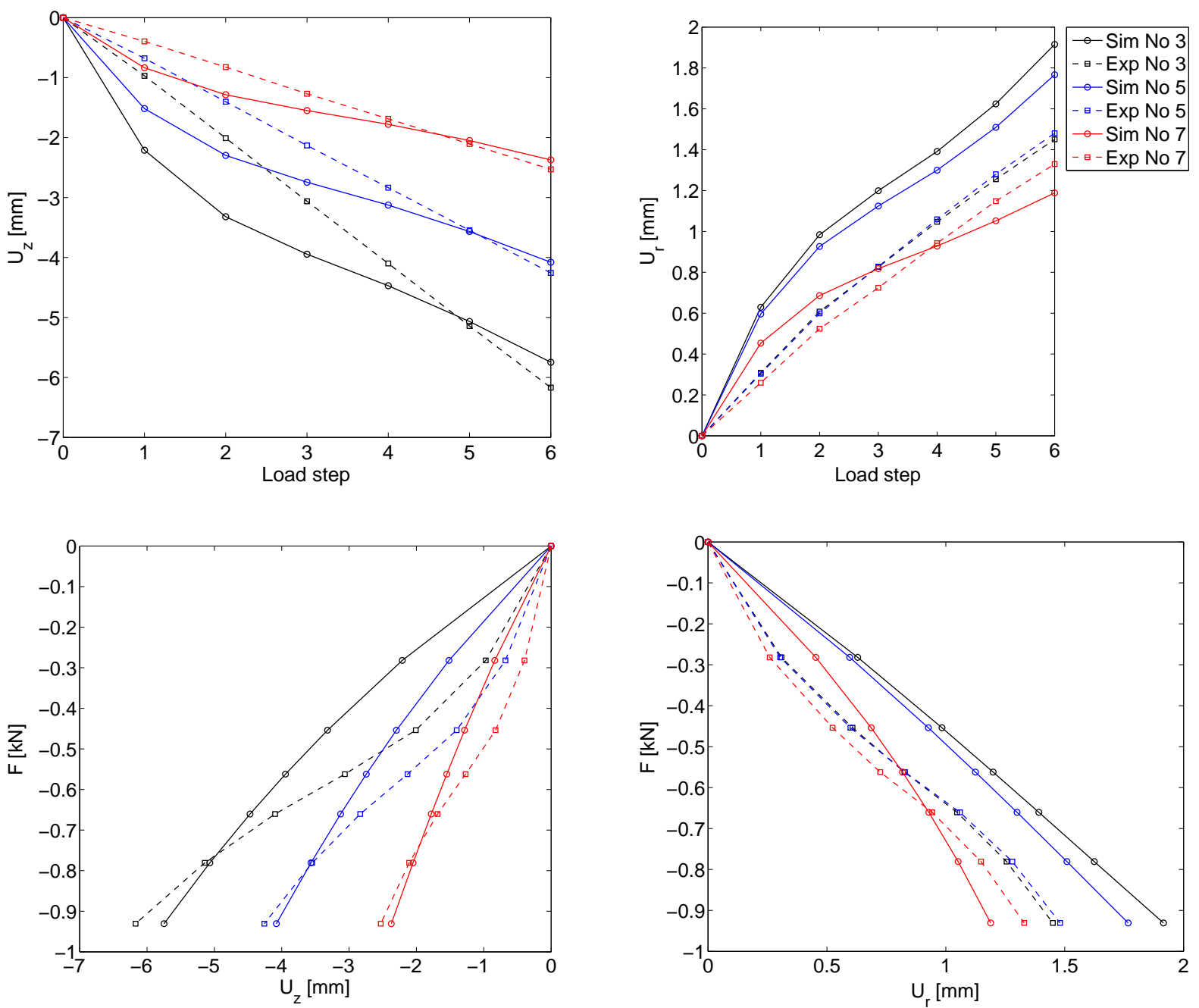

Figure 7.24: End of the identification iteration for test $C$. Displacement versus time curves and force versus displacement curves for three representative identification nodes for the experimental and simulated data. 


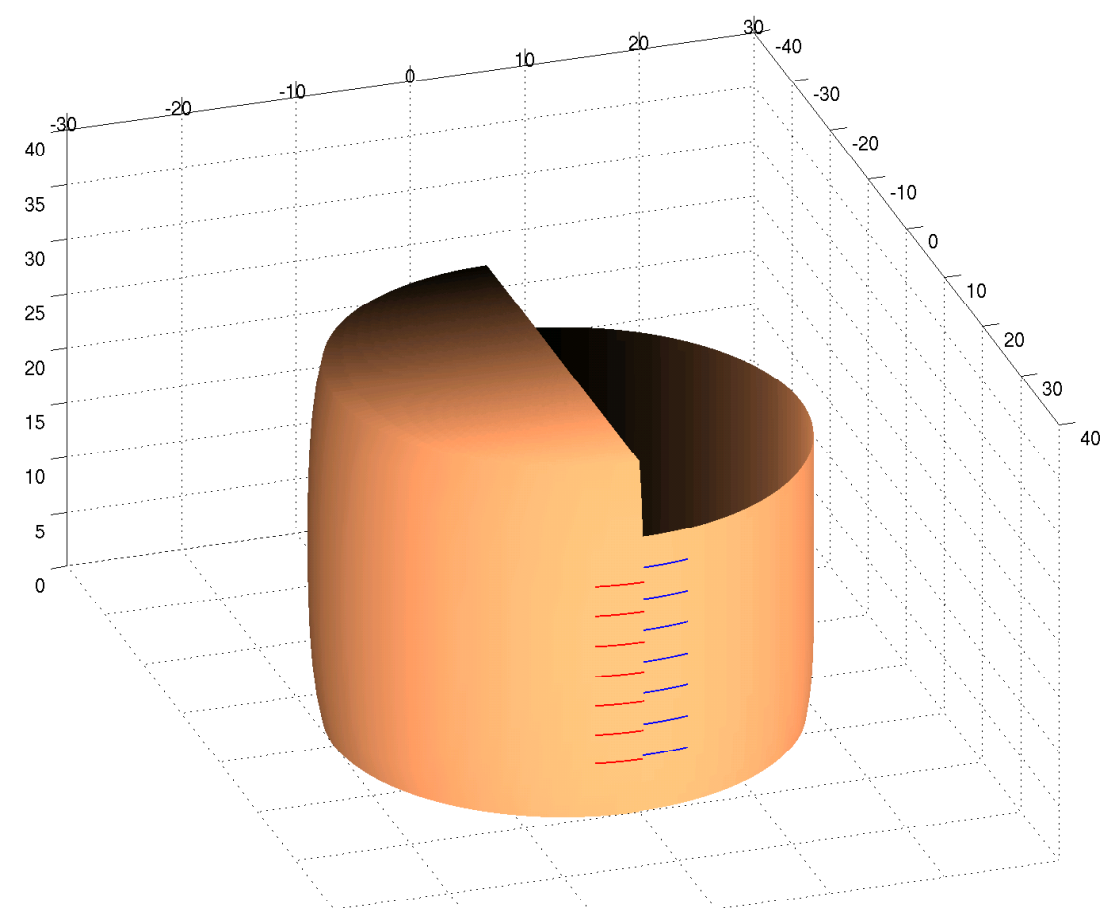

(a) 3th time step.

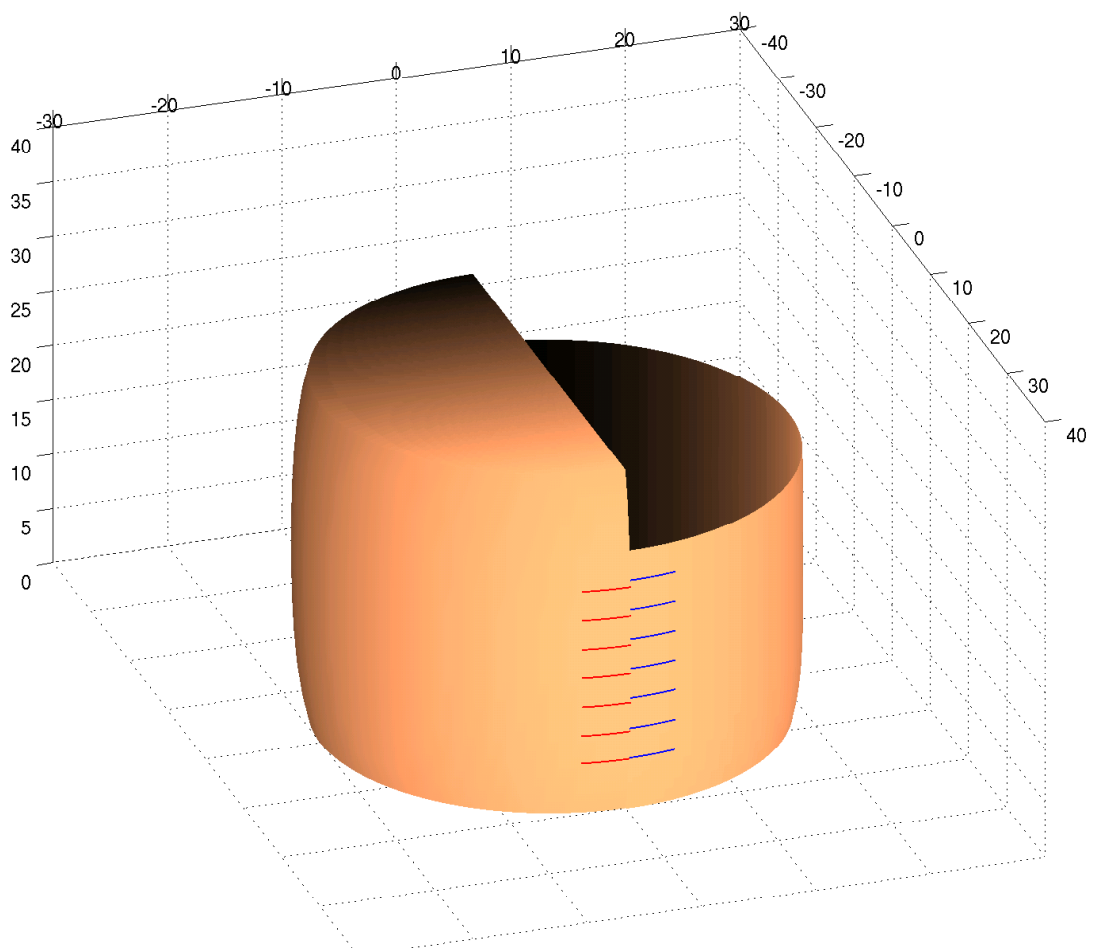

(b) 4th time step.

Figure 7.25: End of the iteration for test $C$ : Left sides of the lateral surface areas: Simulation of the specimen with red marked identification nodes; Right sides of the lateral surface areas: Measured displacement field in the experiment (only a part of the specimen is measured) with blue marked interpolated measured displacements. 


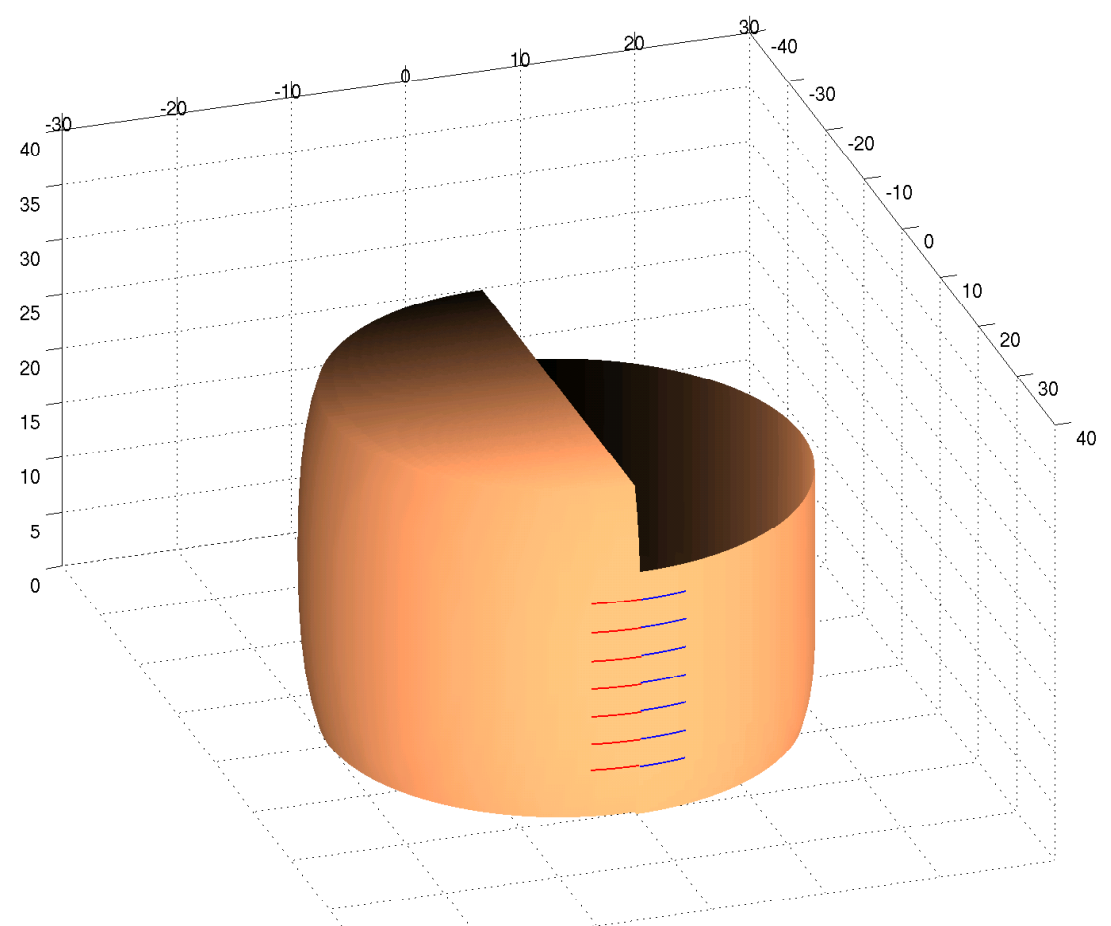

(a) 5th time step.

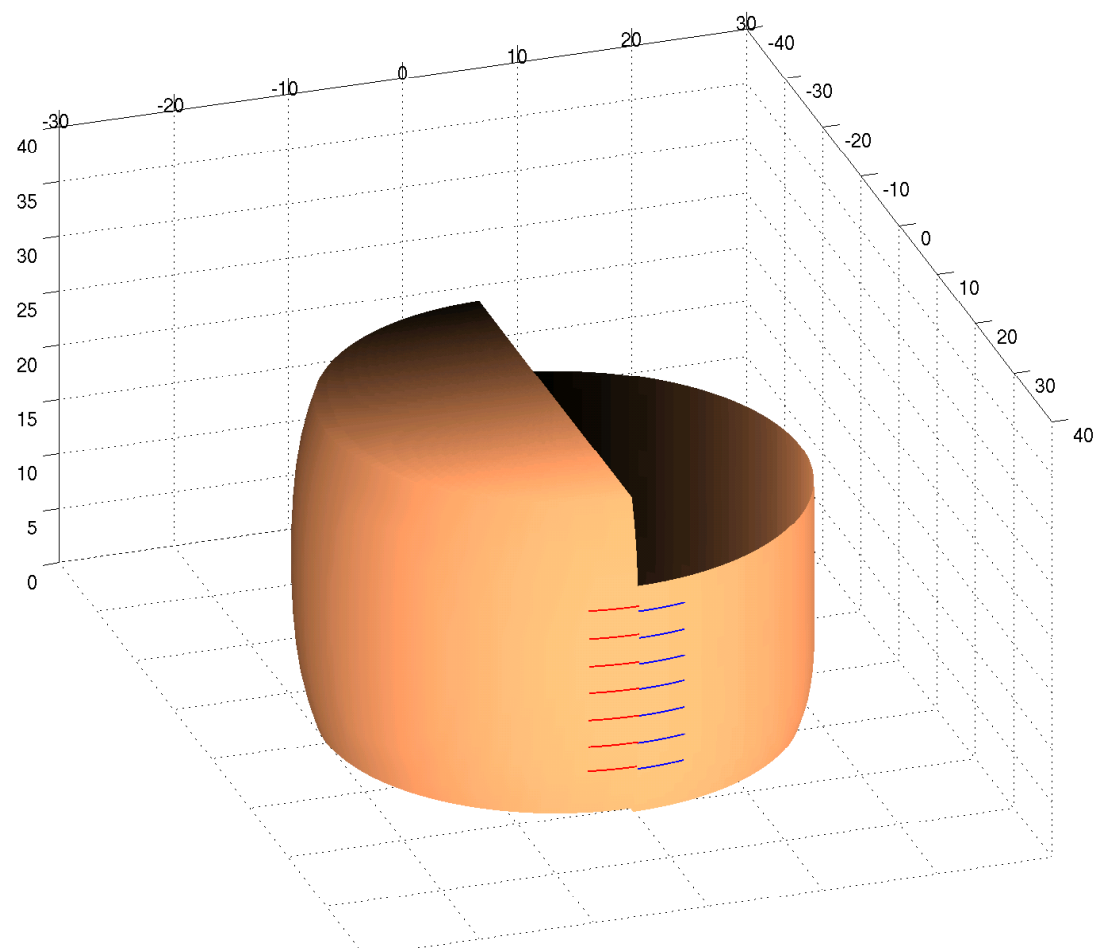

(b) 6th time step.

Figure 7.26: End of the iteration for test $C$ : Left sides of the lateral surface areas: Simulation of the specimen with red marked identification nodes; Right sides of the lateral surface areas: Measured displacement field in the experiment (only a part of the specimen is measured) with blue marked interpolated measured displacements. 
As it can be seen in the verification, the applied viscoelastic material law with the identified parameters is only conditionally appropriate to simulate the mechanical time dependent behavior of the considered polyurethane. The identification has also been carried out with two $\left(N_{T}=2\right)$ and three $\left(N_{T}=3\right)$ terms for the isochoric part [ $\left.\Psi^{\mathrm{eq}}\right]^{\mathrm{dev}}$ of the equilibrium free energy $\Psi^{\mathrm{eq}}$. Thereby, no significant further reduction of the sum of squared differences can be observed. These additional parts of $\left[\Psi^{\mathrm{eq}}\right]^{\mathrm{dev}}$ lead to overparametrization with the instability problems as already mentioned in chapter 6 . Nevertheless, the verification shows that the local minimization of the weighted least squares functional delivers a parameter set which enables to fit the simulations to the respective experiments for all three tests in an average (weighted) way. Moreover, it needs to be mentioned that the identification has been carried out considering a large loading range. Note that the identified parameters are only valid in the considered time ranges of the tests $A$ and $B$ and accordingly at (almost) full relaxation. 


\subsection{Compact polyurethane}

Considering the short- and long time behavior of an elastomer with the aim of describing different relaxation processes, multiple relaxation mechanisms have to be implemented within the constitutive routine. For the parameter identification of these viscoelastic material laws the parameters are usually split into different sets which are each identified individually by one test. In doing so, in order to identify the parameters of the equilibrium part, the experiments have to deliver test data with fully completed stress relaxation. Thereafter the material parameters of the non-equilibrium term(s) often are identified by a single test where the viscous stress relaxation is investigated over a chosen time period while the parameters of the equilibrium term are fixed.

As already mentioned in the previous example by simultaneous identification of all material parameters for different tests an optimal set of parameters in the sense of an optimal adjustment of the constitutive model - being subject to a model error itself - to all tests is achieved. The consideration of all tests in one optimization iteration also leads to an improved adjustment of the imbalances which arise from scattering of experimental data. Moreover the difficulties due to an insufficient number of testing data, which can cause instable or non-unique parameter estimates, might be avoided by taking into account several tests. Then all intended model applications and the respective material parameters can be activated by the experimental data (see Mahnken [40]).

The procedure will be presented for the viscoelastic material law for the example of a compact polyurethane in the following subsections. The material with the trade name PU D44 has a Shore A hardness of 80 and, referring to the data sheet, a very high elongation at break of circa 500\%. Moreover it features a high abrasion resistance and good damping characteristics. For this reason PU D44 has multi-functional application areas and is used for instance as spring-elements, coupling elements, rolls, gaskets and bumpers.

By verification it can be shown that the proposed procedure delivers a very good agreement between experimental and simulated data for all investigated tests. Moreover, as the viscoelastic behavior for long-time intervals is considered, with this approach timeconsuming tests with fully completed stress relaxation are no longer required.

\subsubsection{Experiments}

The experimental data was determined by M. Bosseler of the 'Institute of Resource centered Product Development' under the supervision of Prof. Dr.-Ing. R. Renz, University of Kaiserslautern (see Bosseler et al. [11]).

The material is PU D44 (polyurethane) processed in plates with a thickness of $2.2 \mathrm{~mm}$. The specimens are $25 \mathrm{~mm}$ wide and have a clamping length of $60 \mathrm{~mm}$. For the later identification the tests have to deliver sufficient information about the deformation behavior of the material. Thus for the specimens a geometry is to be chosen which immediately leads to inhomogeneous displacement fields in a tensile test. For this reason a hole of $10 \mathrm{~mm}$ is punched in the middle of the specimens.

The tensile tests are conducted in displacement control. Before the conduction of the first test, the specimen is loaded in six load cycles with the maximal displacement used in the 
later tests. It turned out that this number of load cycles is enough to cause a saturation of damage (Mullins effect). Sufficiently long before the conduction of each test, the specimen is not loaded in order to exclude relaxation effects of the previous test.

In the right photography in figure 7.27 the specimen in a tensile test is shown whereby for the optical deformation analysis by means of image correlation photogrammetry a stochastic pattern has been applied onto the specimen's surface. The test data are the
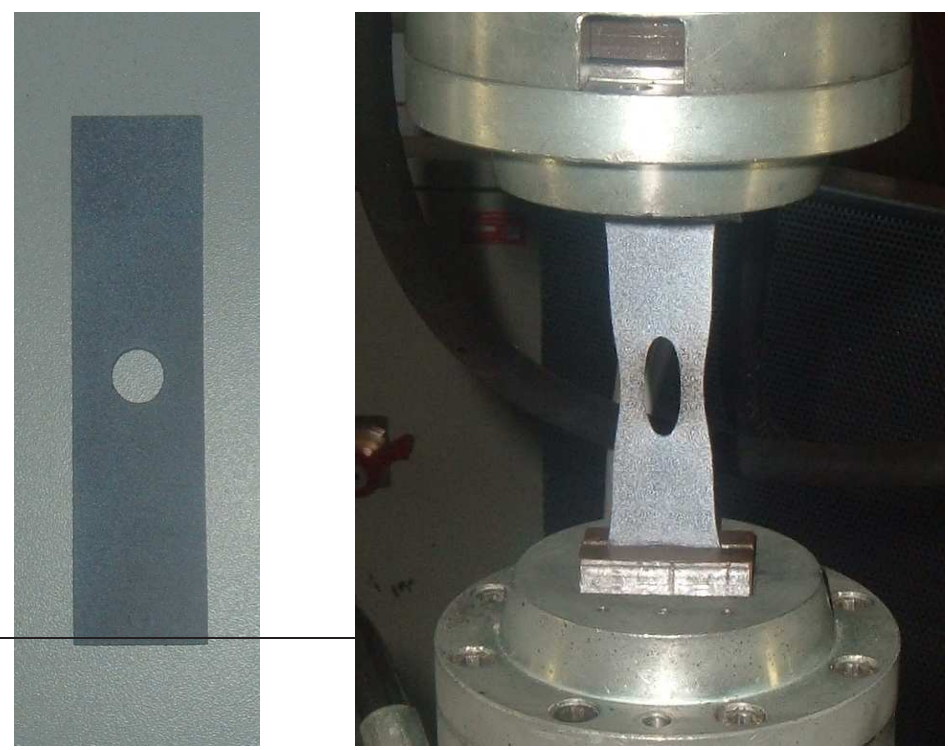

Figure 7.27: Specimen; Specimen with a stochastic pattern in a tensile test.

force-time curves and the three-dimensional displacement field on the surface of the specimen measured by the system ARAMIS ${ }^{4}$. Thereby, using two CCD cameras ${ }^{5}$ with $50 \mathrm{~mm}$ objectives (see the photography in figure 4.1), synchronized stereo images of the specimen are recorded at different time steps of the tests. By means of photogrammetric evaluation procedures for the image processing, the measuring system evaluates the three dimensional displacement field associated with each respective time step.

For the later identification only the (two-dimensional) displacements measured along the longitudinal and transverse direction are used.

Three tests $A, B$, and $C$ performed on one specimen are chosen. The corresponding force time curves are shown in figure 7.28 , whereby $F$ is the total force in loading direction.

Test $A$ is a tension test with one load cycle. The maximal displacement of $30 \mathrm{~mm}$ is applied in $30 \mathrm{~s}$ and held for $6 \mathrm{~s}$. Then the total displacement is reduced in the following $53 \mathrm{~s}$ with a displacement rate of $-0.5 \mathrm{~mm} / \mathrm{s}$. Each second an image of the specimen is taken by the CCD cameras for the displacement measurement.

Test $B$ is a relaxation test. The maximal displacement of $30 \mathrm{~mm}$ is applied in $31 \mathrm{~s}$ and hold for $113 \mathrm{~s}$. Each second an image of the specimen is taken by the CCD cameras for the displacement measurement.

\footnotetext{
${ }^{4}$ Version 5.4 (Gesellschaft für optische Messtechnik mbH)

${ }^{5}$ Type: CCD 1300 (Vosskühler)
} 

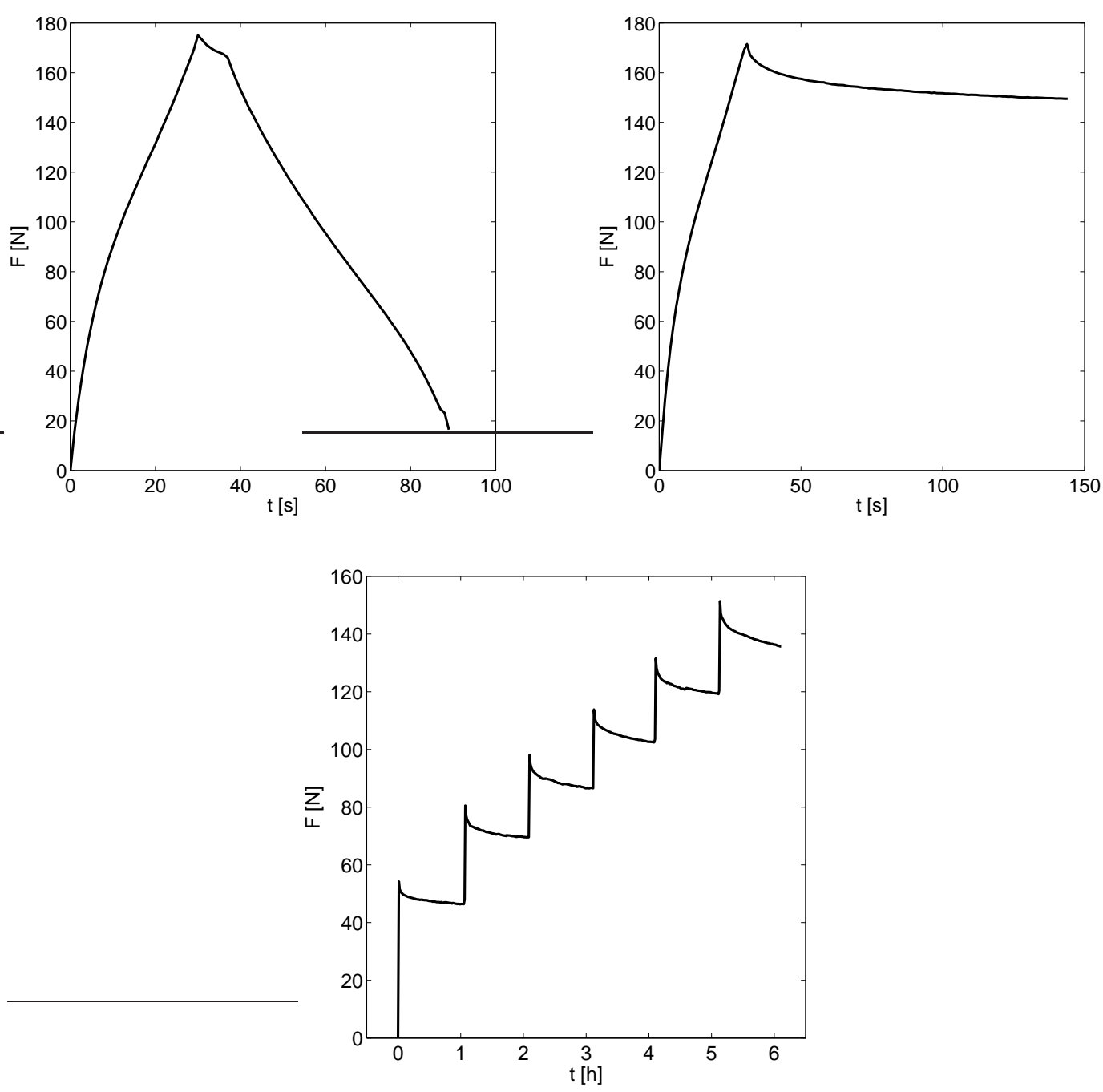

Figure 7.28: Measured force-time curves for test $A$ (top left), $B$ (top right) and $C$ (bottom). These force vs. time data are used for the (force-) control of the corresponding simulations.

Test $C$ reflects six loading steps. The applied total displacements of the specimen are $5.25 \mathrm{~mm}, 10.50 \mathrm{~mm}, 15.75 \mathrm{~mm}, 21.00 \mathrm{~mm}, 26.25 \mathrm{~mm}$ and $31.70 \mathrm{~mm}$. At the end of each loading step an image of the specimen is taken by the CCD cameras for the displacement measurement. The time for the load application between each of these steps is $45 \mathrm{~s}$.

\subsubsection{Interpolation of experimental data}

For the simulation of the tests four FEM discretizations of the specimen with 96, 216, 864 and 2400 eight-node enhanced elements (Q1E9) (see Simo and Armero [69]) are used. Thereby, symmetry conditions in transverse and thickness direction are applied for the FE models and hence only half of the width and half of the thickness is discretized. The boundary conditions for both ending planes normal to the longitudinal direction are chosen in correspondence with the experiments whereby the nodes on one plane are fixed in longitudinal and transverse direction. The nodes on the other plane are fixed in transverse 
direction and in longitudinal direction uniaxial normal forces are applied whereby warping of the plane is prevented. In addition for both planes the edges and straight lines through the nodes along the transverse direction are prevented from curvature and hence can only move parallel in thickness direction. Rigid body motion is excluded. Figure 7.29 shows the entire FEM meshes with the discretizations of the respective corresponding FEM models which serve for the identification routine. In order to reduce the computational costs for

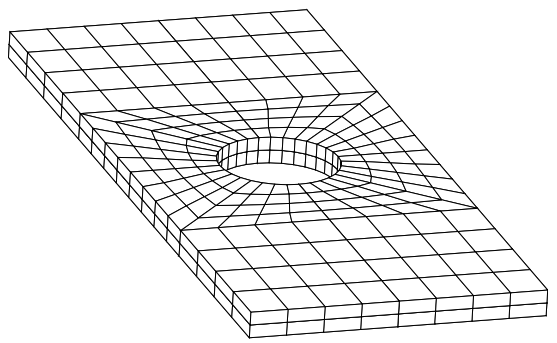

(a) Level one $(4 \times 96$ elements $)$.

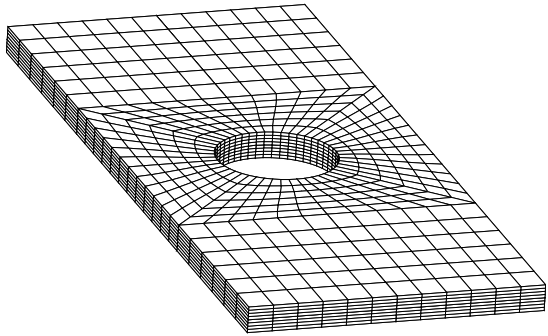

(c) Level three $(4 \times 864$ elements $)$.

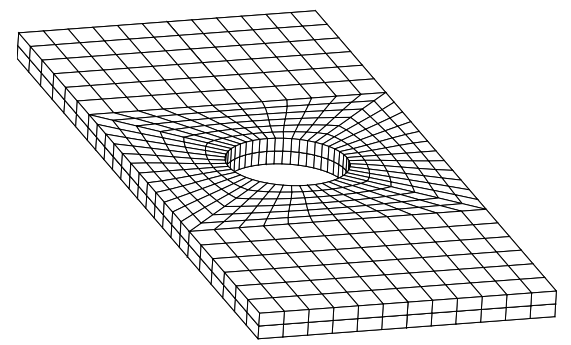

(b) Level two $(4 \times 216$ elements $)$.

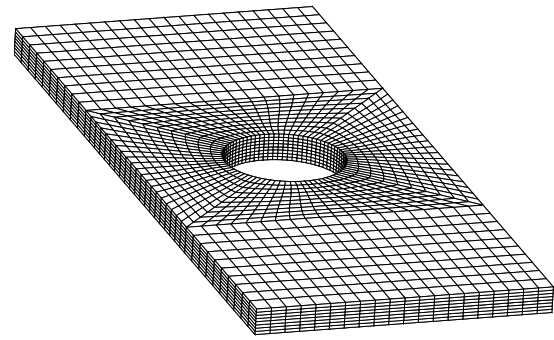

(d) Level four $(4 \times 2400$ elements $)$.

Figure 7.29: Entire FEM models with the discretizations of the respective corresponding FEM models which serve for the identification routine.

the identification routine these FEM discretizations are used in ascending order within a multi level strategy (see Mahnken and Stein [47]). The optimal material parameters determined with the 96-element model at level one are used as initial parameters for the optimization based on the 216-element model at level two and so forth.

Since the coordinates of the measurement points generally do not coincide with the coordinates of the identification nodes, the measured displacements are interpolated linearly on the identification nodes by triangulation (see subsection 4.3.2). For the triangulation the displacements of the three measurement points being nearest to the respective identification node are used. As only half of the width is discretized, the measured displacements of the opposite side of the longitudinal centerline are mirrored onto the considered side and two separate triangulations for both displacement fields on the identification nodes are performed. For each identification node and displacement direction the mean value of these two data sets is taken into account. 
In order to make the three tests $A, B$, and $C$ comparable for the later identification identical identification nodes are chosen throughout for all three tests.

\subsubsection{Least squares problem}

In order to identify the parameters for the tests $A, B$, and $C$ simultaneously, the least squares approach in equation (4.8) is modified, namely

$$
\begin{aligned}
f(\boldsymbol{\kappa})= & \frac{1}{2} \sum_{i=1}^{N_{g}}[\underbrace{\sum_{j=1}^{T_{A}}\left[\boldsymbol{W}_{i j} \cdot\left[\overline{\boldsymbol{u}}_{i j}(\boldsymbol{\kappa})-\overline{\boldsymbol{u}}_{i j}^{e x p}\right]\right]^{2}}_{A}+\underbrace{\sum_{k=1}^{T_{B}}\left[\boldsymbol{W}_{i k} \cdot\left[\overline{\boldsymbol{u}}_{i k}(\boldsymbol{\kappa})-\overline{\boldsymbol{u}}_{i k}^{e x p}\right]\right]^{2}}_{B} \\
& +\underbrace{\left.\sum_{l=1}^{T_{C}}\left[\boldsymbol{W}_{i l} \cdot\left[\overline{\boldsymbol{u}}_{i l}(\boldsymbol{\kappa})-\overline{\boldsymbol{u}}_{i l}^{e x p}\right]\right]^{2}\right]}_{C} .
\end{aligned}
$$

whereby $\boldsymbol{W}_{i j}, \boldsymbol{W}_{i k}$, and $\boldsymbol{W}_{i l}$ are weighting matrices with $\boldsymbol{W}_{i j}=\operatorname{diag}\left(\left[W_{i j}\right]_{1},\left[W_{i j}\right]_{2}\right)$, $\boldsymbol{W}_{i k}=\operatorname{diag}\left(\left[W_{i k}\right]_{1},\left[W_{i k}\right]_{2}\right)$, and $\boldsymbol{W}_{i l}=\operatorname{diag}\left(\left[W_{i l}\right]_{1},\left[W_{i l}\right]_{2}\right)$. Thereby $\left[W_{i j}\right]_{1},\left[W_{i k}\right]_{1}$, and $\left[W_{i l}\right]_{1}$ are the weights for the respective displacements in longitudinal direction whereas $\left[W_{i j}\right]_{2},\left[W_{i k}\right]_{2}$, and $\left[W_{i l}\right]_{2}$ are the weights for the respective displacements in transverse direction. For the simulation of the displacement fields $\overline{\boldsymbol{u}}_{i j}(\boldsymbol{\kappa}), \overline{\boldsymbol{u}}_{i k}(\boldsymbol{\kappa})$ and $\overline{\boldsymbol{u}}_{i l}(\boldsymbol{\kappa})$ the FEM simulation is force-controlled based on the measured force signals shown in figure 7.28. Moreover, $N_{g}$ denotes the number of identification nodes which is identical for all tests. Nevertheless $N_{g}$ is dependent on the discretization for the different FE models in the identification process as described in subsection 7.3.2. The number of identification nodes is at level one: $N_{g}=N_{g}^{l e v_{1}}=19$. At the levels two $\left(N_{g}=N_{g}^{l e v_{2}}=43\right)$ and three $\left(N_{g}=N_{g}^{\text {lev }_{3}}=43\right)$ the discretizations only differ in the number of elements in thickness direction. For level four the discretization yields $N_{g}=N_{g}^{l e v_{4}}=116$.

In test $A$ the number of time steps is $T_{A}=89$ with a time step length of $\Delta t=1 \mathrm{~s}$. For $B$ the number of time steps corresponds to $T_{B}=144$ with a time step length of $\Delta t=1 \mathrm{~s}$. Within test $C$ we account for a total number of 489 time steps with a time step length of $\Delta t=45 \mathrm{~s}$. However, not all of these time steps contribute to the least squares sum. Due to the fact that the clamping displacements are fixed during the individual loading steps use is made of the displacement field measured at the end of each loading step for 77 supporting points so that $T_{C}=77 \times 6=462$. Although the first 76 supporting points are not exact measurement data, they can be used to specify these displacement fields with a sufficient accuracy. This is also reflected by the later on presented displacement curves for the short-time relaxation test $B$. Moreover, this small reduction of accuracy is balanced by smaller weights $\boldsymbol{W}_{i l}$ on the first 76 supporting points in each loading step (see below). The displacements calculated by FEM $\overline{\boldsymbol{u}}_{i j}(\boldsymbol{\kappa}), \overline{\boldsymbol{u}}_{i k}(\boldsymbol{\kappa}), \overline{\boldsymbol{u}}_{i l}(\boldsymbol{\kappa})$ and the measured displacements $\overline{\boldsymbol{u}}_{i j}^{\text {exp }}, \overline{\boldsymbol{u}}_{i k}^{\text {exp }}, \overline{\boldsymbol{u}}_{i l}^{\text {exp }}$ have, respectively, two displacement directions. Thus for the FEM discretization with 2400 elements the number of terms in the least square sum adds up to $116 \times[89+144+462] \times 2=161240$ per iteration step of the optimization algorithm. By taking into account that the displacement data of the experiments has been mirrored, the total number of displacement data compared within the optimization algorithm is $2 \times 161240=322480$ per iteration step. 
The longitudinal displacements are much larger compared with the transverse displacements. In addition, we want to emphasize some particular time steps of the tests as well as the tests relative to each other. Accordingly, the matrices $\boldsymbol{W}_{i j}, \boldsymbol{W}_{i k}$, and $\boldsymbol{W}_{i l}$ referring to each time step of the tests are incorporated.

For all time steps $1 \leq j \leq T_{A}$ and all identification nodes $1 \leq i \leq N_{g}$ within test $A$ we assume

$$
\boldsymbol{W}_{i j}=\operatorname{diag}(1,8) .
$$

The weights for test $B$ are

$$
\boldsymbol{W}_{i k}=\operatorname{diag}(1,8) \quad \forall 1 \leq i \leq N_{g} \wedge 1 \leq k \leq 31
$$

and

$$
\boldsymbol{W}_{i k}=\operatorname{diag}(2,8) \quad \forall 1 \leq i \leq N_{g} \wedge 32 \leq k \leq T_{B} .
$$

The weights for test $\mathrm{C}$ are for the first 76 supporting points in each loading step

$$
\boldsymbol{W}_{i l}=\operatorname{diag}(1,4) \quad \forall 1 \leq i \leq N_{g} \wedge l \in[77,154,231,308,385,462]
$$

and at the end of each loading step

$$
\begin{aligned}
\boldsymbol{W}_{i l}=\operatorname{diag}(2,8) \quad \forall 1 \leq i \leq N_{g} & \wedge 1 \leq l \leq T_{C} \\
& \wedge l \notin[77,154,231,308,385,462] .
\end{aligned}
$$

\subsubsection{Choice of the model structure}

Apparently, the structure of the viscoelastic model has to be adjusted for the simulation of the tested material. The comparison of tests $A, B$, and $C$ shows that the material exhibits two defined relaxation processes in the considered time ranges. Thus two relaxation terms $\mathrm{k}=\{1,2\}$ are implemented. Secondly the number of terms in the sum of the Ogdenmodels for the equilibrium and the two non-equilibrium terms has to be chosen. From the authors experience for the considered deformation ranges the implementation of more than one term will not lead to a significant improvement (lowering of the least squares sum in parameter identification) but might lead to an instable or non-unique solution for the identified material parameters (see also chapter 6). In addition, the parameters for the extra terms implicate extra computational costs. Hence for the equilibrium term, for the first relaxation module and for the second relaxation module we accept $\mathrm{N}_{\mathrm{T}}=\mathrm{N}_{\mathrm{T}}^{\mathrm{k}=1}=$ $\mathrm{N}_{\mathrm{T}}^{\mathrm{k}=2}=1$.

Hence, the set of material parameters for the identification problem is

$$
\boldsymbol{\kappa}=\left[\left[\mu_{\text {eq }}\right]_{1},\left[\alpha_{\text {eq }}\right]_{1}, \kappa_{\text {eq }},\left[\mu_{\text {neq }}^{\mathrm{k}=1}\right]_{1},\left[\alpha_{\text {neq }}^{\mathrm{k}=1}\right]_{1}, \kappa_{\text {neq }}^{\mathrm{k}=1}, \hat{\tau}^{\mathrm{k}=1},\left[\mu_{\text {neq }}^{\mathrm{k}=2}\right]_{1},\left[\alpha_{\text {neq }}^{\mathrm{k}=2}\right]_{1}, \kappa_{\text {neq }}^{\mathrm{k}=2}, \hat{\tau}^{\mathrm{k}=2}\right]^{\mathrm{T}} .
$$

For simplicity, the subindex for the sum terms mentioned above is omitted

$$
\boldsymbol{\kappa}=\left[\mu_{\text {eq }}, \alpha_{\text {eq }}, \kappa_{\text {eq }}, \mu_{\text {neq }}^{\mathrm{k}=1}, \alpha_{\text {neq }}^{\mathrm{k}=1}, \kappa_{\text {neq }}^{\mathrm{k}=1}, \hat{\tau}^{\mathrm{k}=1}, \mu_{\text {neq }}^{\mathrm{k}=2}, \alpha_{\text {neq }}^{\mathrm{k}=2}, \kappa_{\text {neq }}^{\mathrm{k}=2}, \hat{\tau}^{\mathrm{k}=2}\right]^{\mathrm{T}} .
$$

The rheological network of the chosen model structure might be represented in the form of two Maxwell elements in parallel with an equilibrium spring as shown in figure 7.30. The springs stand for the respective nonlinear functions of the corresponding strains $\boldsymbol{b}, \boldsymbol{b}_{\mathrm{e}}^{\mathrm{k}=1}$ and $\boldsymbol{b}_{\mathrm{e}}^{\mathrm{k}=2}$. The respective parameter sets which are associated directly to these nonlinear functions are assigned to the springs in figure 7.30. 


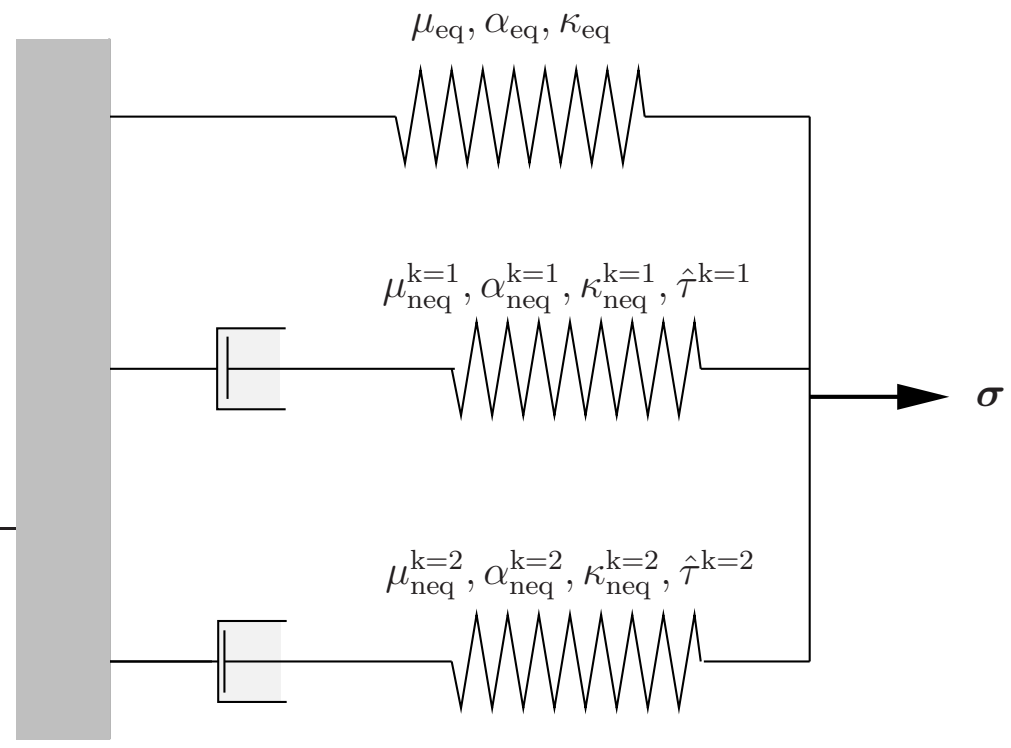

Figure 7.30: Rheological network of the model structure.

\subsubsection{Parameter identification process}

Because of the non-convexity of the objective function and the existence of (many) local minima the hybrid method of application of the Levenberg-Marquard method to find different local minima for stochastically chosen start parameter sets is used (see section 4.4).

First level (FEM model with 96 elements): In order to save computing time the above described hybrid method described above is mainly applied in the first level, whereby the FEM model with 96 elements is used for the identification routine. As an example, table 7.7 shows a part of the iteration leading to the smallest of all investigated local minima in $\boldsymbol{\kappa}=\boldsymbol{\kappa}_{l m_{1}}^{l e v_{1}}$. Hereby, the weighted sum of squared differences for the start of the iteration $f_{\text {start }}^{l e v_{1}}$ and for the end of iteration $f_{l m_{1}}^{l e v_{1}}$ (after seven iteration steps) have the relation $f_{l m_{1}}^{l e v_{1}} \approx 0.0502 f_{\text {start }_{1}}^{\text {lev }}$.

Table 7.7: Example of a start parameter set and end parameter set $\kappa_{l m_{1}}^{l e v_{1}}$ within the iteration of the first level.

\begin{tabular}{|l||l|l|l|l|l|l|l|l|l|l|l|}
\hline & $\begin{array}{l}\mu_{\text {eq }} \\
{[\mathrm{MPa}]}\end{array}$ & $\begin{array}{l}\alpha_{\mathrm{eq}} \\
{[-]}\end{array}$ & $\begin{array}{l}\kappa_{\mathrm{eq}} \\
{[\mathrm{MPa}]}\end{array}$ & $\begin{array}{l}\mu_{\text {neq }}^{\mathrm{k}=1} \\
{[\mathrm{MPa}]}\end{array}$ & $\begin{array}{l}\alpha_{\text {neq }}^{\mathrm{k}=1} \\
{[-]}\end{array}$ & $\begin{array}{l}\kappa_{\text {neq }}^{\mathrm{k}=1} \\
{[\mathrm{MPa}]}\end{array}$ & $\begin{array}{l}\hat{\tau}^{\mathrm{k}=1} \\
{[\mathrm{~s}]}\end{array}$ & $\begin{array}{l}\mu_{\text {neq }}^{\mathrm{k}=2} \\
{[\mathrm{MPa}]}\end{array}$ & $\begin{array}{l}\alpha_{\text {neq }}^{\mathrm{k}=2} \\
{[-]}\end{array}$ & $\begin{array}{l}\kappa_{\text {neq }}^{\mathrm{k}=2} \\
{[\mathrm{MPa}]}\end{array}$ & $\begin{array}{l}\hat{\tau}^{\mathrm{k}=2} \\
{[\mathrm{~s}]}\end{array}$ \\
\hline \hline Start & 1.4968 & 2.6458 & 7.2937 & 2.9747 & 1.5146 & 0.1 & 4.2497 & 2 & 2 & 0.1 & 9814.0 \\
\hline End $\left(\kappa_{l m_{1}}^{\text {lev }}\right)$ & 1.6136 & 2.4982 & 9.1494 & 3.0075 & 1.5718 & 0.1527 & 4.1338 & 1.9504 & 1.2481 & 0.6154 & 9846.8 \\
\hline
\end{tabular}

The parameter sets $\boldsymbol{\kappa}_{l m_{i}}^{l e v_{1}}$ for the four local minima with the smallest weighted sum of squared errors determined at level one are listed in table 7.8. Thereby, as the least squares functional is weighted we do not designate the values of the weighted sum of squared errors $f_{l m_{i}}^{l e v_{i}}$. These errors for different parameter sets are compared by referring to $f_{l m_{1}}^{l e v_{1}}$ for the parameter set $\boldsymbol{\kappa}_{l m_{1}}^{l e v_{1}}$ by defining $\Delta f_{l m_{i}}^{l e v_{1}}=100\left(f_{l m_{i}}^{l e v_{1}}-f_{l m_{1}}^{l e v_{1}}\right) / f_{l m_{1}}^{l e v_{1}}$.

Second, third, and fourth level (FEM models with 216, 814, and 2400 elements): At the end of the first level the two sets of parameters $\boldsymbol{\kappa}_{l m_{1}}^{l e v_{1}}$ and $\boldsymbol{\kappa}_{l m_{2}}^{l e v_{1}}$, which generate the 
Table 7.8: Determined local minima at level one

\begin{tabular}{|c|c||c|c|c|c|c|c|l|l|l|l|l|}
\hline & $\Delta f_{l m_{i}}^{l e v_{1}}$ & $\begin{array}{l}\mu_{\mathrm{eq}} \\
{[\mathrm{MPa}]}\end{array}$ & $\begin{array}{l}\alpha_{\mathrm{eq}} \\
{[-]}\end{array}$ & $\begin{array}{l}\kappa_{\text {eq }} \\
{[\mathrm{MPa}]}\end{array}$ & $\begin{array}{l}\mu_{\text {neq }}^{\mathrm{k}=1} \\
{[\mathrm{MPa}]}\end{array}$ & $\begin{array}{l}\alpha_{\text {neq }}^{\mathrm{k}=1} \\
{[-]}\end{array}$ & $\begin{array}{l}\kappa_{\text {neq }}^{\mathrm{k}=1} \\
{[\mathrm{MPa}]}\end{array}$ & $\begin{array}{l}\hat{\tau}^{\mathrm{k}=1} \\
{[\mathrm{~s}]}\end{array}$ & $\begin{array}{l}\mu_{\text {neq }}^{\mathrm{k}=2} \\
{[\mathrm{MPa}]}\end{array}$ & $\begin{array}{l}\alpha_{\text {neq }}^{\mathrm{k}=2} \\
{[-]}\end{array}$ & $\begin{array}{l}\kappa_{\text {neq }}^{\mathrm{k}=2} \\
{[\mathrm{MPa}]}\end{array}$ & $\begin{array}{l}\hat{\tau}^{\mathrm{k}=2} \\
{[\mathrm{~s}]}\end{array}$ \\
\hline \hline $\boldsymbol{\kappa}_{l m_{1}}^{l e v_{1}}$ & 0 & 1.6136 & 2.4982 & 9.1494 & 3.0075 & 1.5718 & 0.1527 & 4.1338 & 1.9504 & 1.2481 & 0.6154 & 9846.8 \\
\hline $\boldsymbol{\kappa}_{l m_{2}}^{l e v_{1}}$ & $2.2 \%$ & 1.2686 & 2.7052 & 9.7860 & 2.0348 & 1.6451 & 0.0411 & 6.7217 & 3.1622 & 0.9609 & 0.1008 & 12956.9 \\
\hline $\boldsymbol{\kappa}_{l m_{3}}^{l e m_{1}}$ & $3.6 \%$ & 1.4331 & 2.5806 & 9.8934 & 2.0186 & 1.5051 & 0.2380 & 7.7610 & 2.8944 & 0.9530 & 0.2323 & 11765.0 \\
\hline $\boldsymbol{\kappa}_{l m_{4}}^{l e v_{1}}$ & $4.8 \%$ & 1.9386 & 2.3250 & 9.2410 & 2.7570 & 1.1119 & 0.0741 & 7.7249 & 1.6730 & 1.1775 & 0.4444 & 7497.17 \\
\hline
\end{tabular}

smallest sums of squared differences out of all investigated local minima, are chosen as start parameters for the following level whereby $\boldsymbol{\kappa}_{l m_{1}}^{l e v_{1}}$ remains to feature the parameter set with the smaller weighted sum of squared errors. The following identifications with the FEM models with 216, 864, and 2400 elements finally yield the optimal solution shown in table 7.9. Although the number of identification nodes at level four is circa 2.7 times

Table 7.9: Multi level iteration for $\kappa_{l m_{1}}^{l e v_{1}}$.

\begin{tabular}{|c||c|c|c|c|c|c|c|c|c|c|c|}
\hline & $\begin{array}{l}\mu_{\mathrm{eq}} \\
{[\mathrm{MPa}]}\end{array}$ & $\begin{array}{l}\alpha_{\mathrm{eq}} \\
{[-]}\end{array}$ & $\begin{array}{l}\kappa_{\mathrm{eq}} \\
{[\mathrm{MPa}]}\end{array}$ & $\begin{array}{l}\mu_{\text {neq }}^{\mathrm{k}=1} \\
{[\mathrm{MPa}]}\end{array}$ & $\begin{array}{l}\alpha_{\text {neq }}^{\mathrm{k}=1} \\
{[-]}\end{array}$ & $\begin{array}{l}\kappa_{\text {neq }}^{\mathrm{k}=1} \\
{[\mathrm{MPa}]}\end{array}$ & $\begin{array}{l}\hat{\tau}^{\mathrm{k}=1} \\
{[\mathrm{~s}]}\end{array}$ & $\begin{array}{l}\mu_{\text {neq }}^{\mathrm{k}=2} \\
{[\mathrm{MPa}]}\end{array}$ & $\begin{array}{l}\alpha_{\text {neq }}^{\mathrm{k}=2} \\
{[-]}\end{array}$ & $\begin{array}{l}\kappa_{\text {neq }}^{\mathrm{k}=2} \\
{[\mathrm{MPa}]}\end{array}$ & $\begin{array}{l}\hat{\tau}^{\mathrm{k}=2} \\
{[\mathrm{~s}]}\end{array}$ \\
\hline \hline$\kappa_{l m_{1}}^{\text {lev }}$ & 1.6136 & 2.4982 & 9.1494 & 3.0075 & 1.5718 & 0.1527 & 4.1338 & 1.9504 & 1.2481 & 0.6154 & 9846.8 \\
\hline$\kappa_{l m_{1}}^{l e v_{2}}$ & 1.6503 & 2.4757 & 9.0033 & 3.0314 & 1.5873 & 0.1628 & 4.4070 & 2.0290 & 1.2012 & 0.6300 & 9823.86 \\
\hline$\kappa_{l m_{1}}^{\text {lev }}$ & 1.6487 & 2.4780 & 8.9790 & 3.0246 & 1.5601 & 0.1678 & 4.5127 & 2.0597 & 1.1847 & 0.6408 & 9810.65 \\
\hline$\kappa_{l m_{1}}^{\text {lev }}$ & 1.6618 & 2.4688 & 8.9079 & 3.0272 & 1.5599 & 0.1694 & 4.5530 & 2.0773 & 1.1795 & 0.6390 & 9806.90 \\
\hline
\end{tabular}

higher than at level three, the change between these levels does not have a big influence on the weighted sum of squared differences. Hereby, the weighted sum of squared differences for the start of the iteration $f_{\text {start }_{1}}^{l e v_{4}}$ and for the end of iteration $f_{l m_{1}}^{l e v_{4}}$ (after two iteration steps) have the relation $f_{l m_{1}}^{l e v_{1}} \approx 0.9935 f_{\text {start }_{1}}^{\text {lev }}$. After four more iteration steps no further (significant) reduction of $f^{l e v_{1}}$ can be observed.

It is to be mentioned that different weights as shown in subsection 7.3 .3 will lead to a different solution. The later verification by comparison of the calculated displacements with the parameter set $\boldsymbol{\kappa}_{l m_{1}}^{l e v_{4}}$ and measured displacements for the identification nodes gives information about the error pattern of the individual parts of the sum of squared differences. This allows to review if the weighting of the three tests, time steps and displacement directions among each other has been chosen reasonably.

Secondly, in the identification iteration some instabilities occurred (see also section 4.5). The consequence of these instabilities is that there exist several parameter sets which lead to a insignificantly higher least squares sum. In order to evaluate the significance for the quality of the solution, the correlation matrix for the optimal parameters shown in table 7.10 (see also equations (4.15) and (4.16)) is considered.

The absolute values of most of the correlation numbers are small. There are three categories of parameters which correlate.

Firstly, the pairs of parameters $\left(\mu_{\mathrm{eq}}, \alpha_{\mathrm{eq}}\right),\left(\mu_{\mathrm{neq}}^{\mathrm{k}=1}, \alpha_{\text {neq }}^{\mathrm{k}=1}\right)$ and $\left(\mu_{\text {neq }}^{\mathrm{k}=2}, \alpha_{\text {neq }}^{\mathrm{k}=2}\right)$ have correlation numbers close to \pm 1 . This is due to the structure of the Ogden-model which can lead to overparametrization even for only one deviatoric term within the corresponding part 
Table 7.10: Correlation matrix for the set of optimal parameters $\kappa_{l m_{1}}^{l e v_{4}}$.

\begin{tabular}{|c||l|l|l|l|l|l|l|l|l|l|l|}
\hline & $\mu_{\text {eq }}$ & $\alpha_{\text {eq }}$ & $\mu_{\text {neq }}^{\mathrm{k}=1}$ & $\alpha_{\text {neq }}^{\mathrm{k}=1}$ & $\kappa_{\text {eq }}$ & $\kappa_{\text {neq }}^{\mathrm{k}=1}$ & $\hat{\tau}^{\mathrm{k}=1}$ & $\mu_{\text {neq }}^{\mathrm{k}=2}$ & $\alpha_{\text {neq }}^{\mathrm{k}=2}$ & $\kappa_{\text {neq }}^{\mathrm{k}=2}$ & $\hat{\tau}^{\mathrm{k}=2}$ \\
\hline \hline$\mu_{\mathrm{eq}}$ & 1.000 & -0.985 & 0.175 & -0.175 & 0.304 & 0.035 & -0.194 & -0.720 & 0.476 & -0.246 & -0.876 \\
\hline$\alpha_{\mathrm{eq}}$ & -0.985 & 1.000 & -0.111 & 0.110 & -0.388 & -0.034 & 0.135 & 0.802 & -0.592 & 0.344 & 0.795 \\
\hline$\mu_{\text {neq }}^{\mathrm{k}=1}$ & 0.175 & -0.111 & 1.000 & -1.000 & -0.009 & 0.205 & -0.909 & 0.268 & -0.403 & 0.113 & -0.409 \\
\hline$\alpha_{\text {neq }}^{\mathrm{k}=1}$ & -0.175 & 0.110 & -1.000 & 1.000 & 0.009 & -0.216 & 0.901 & -0.270 & 0.405 & -0.114 & 0.409 \\
\hline$\kappa_{\text {eq }}$ & 0.304 & -0.388 & -0.009 & 0.009 & 1.000 & 0.074 & 0.004 & -0.421 & 0.429 & -0.937 & -0.236 \\
\hline$\kappa_{\text {neq }}^{\mathrm{k}=1}$ & 0.035 & -0.034 & 0.205 & -0.216 & 0.074 & 1.000 & -0.020 & -0.015 & 0.010 & -0.128 & -0.058 \\
\hline$\hat{\tau}^{\mathrm{k}=1}$ & -0.194 & 0.135 & -0.909 & 0.901 & 0.004 & -0.020 & 1.000 & -0.199 & 0.325 & -0.100 & 0.396 \\
\hline$\mu_{\text {neq }}^{\mathrm{k}=2}$ & -0.720 & 0.802 & 0.268 & -0.270 & -0.421 & -0.015 & -0.199 & 1.000 & -0.952 & 0.519 & 0.327 \\
\hline$\alpha_{\text {neq }}^{\mathrm{k}=2}$ & 0.476 & -0.592 & -0.403 & 0.405 & 0.429 & 0.010 & 0.325 & -0.952 & 1.000 & -0.576 & -0.031 \\
\hline$\kappa_{\text {neq }}^{\mathrm{k}=2}$ & -0.246 & 0.344 & 0.113 & -0.114 & -0.937 & -0.128 & -0.100 & 0.519 & -0.576 & 1.000 & 0.104 \\
\hline$\hat{\tau}^{\mathrm{k}=2}$ & -0.876 & 0.795 & -0.409 & 0.409 & -0.236 & -0.058 & 0.396 & 0.327 & -0.031 & 0.104 & 1.000 \\
\hline
\end{tabular}

of the energy. Since only parameters within the particular deviatoric terms are affected, these correlations will not have a significant influence on the quality of the solution.

Secondly, the parameters for the deviatoric part of the first relaxation module strongly correlate with the first relaxation time $\left[\left(\mu_{\text {neq }}^{\mathrm{k}=1}, \hat{\tau}^{\mathrm{k}=1}\right)\right.$ and $\left.\left(\alpha_{\mathrm{neq}}^{\mathrm{k}=1}, \hat{\tau}^{\mathrm{k}=1}\right)\right]$ which arises from different facts: On the one hand, a constitutive law cannot perfectly describe all characteristics of a material and these parameters have to fit to the viscoelastic behavior in one load cycle (test $A$ ) and a relaxation (test $B$ ) at the same time. On the other hand, experimental data is always subject to errors (measurement errors, scattering), especially for time-dependent material behavior, which makes it rather difficult to determine one fixed relaxation time $\left(\hat{\tau}^{\mathrm{k}=1}\right)$ for multiple affected tests.

Thirdly, the parameters for the deviatoric part of the equilibrium part strongly correlate with the second relaxation time $\left[\left(\mu_{\mathrm{eq}}, \hat{\tau}^{\mathrm{k}=2}\right)\right.$ and $\left.\left(\alpha_{\mathrm{eq}}, \hat{\tau}^{\mathrm{k}=2}\right)\right]$. In addition the compression modules $\left(\kappa_{\text {eq }}, \kappa_{\text {neq }}^{\mathrm{k}=2}\right)$ and the parameters $\left(\alpha_{\text {eq }}, \mu_{\text {neq }}^{\mathrm{k}=2}\right)$ for the equilibrium part and the second relaxation part have high correlation numbers. Within the experimental data of test $C$ six loading steps are considered each one delivering contributions to identify the second relaxation time. Since primarily the relaxation of the second relaxation term leads to the identification of the equilibrium parameters, the above mentioned difficulty of determining one fixed relaxation time for multiple affected tests (loading steps) causes this instability. The high variances of $\hat{\tau}^{\mathrm{k}=2}$ for the different local minima at level one (see table 7.9) are the result of this instability.

Remark 7.3.1 Since the scope of this work is the parameter identification for viscoelasticity at long-time intervals under tension, the tests might have too few information about the (viscoelastic) behavior under compression (only parts around the hole are loaded under compression). The validity of the identified set of parameters for compression-dominated loading, however, is not investigated in this work but rather constitutes future work.

Remark 7.3.2 Since the order of magnitude of the parameters differs, a parameter weight $\boldsymbol{V}^{(j)}$ is employed to the calculation of the iteration step (see also equation 4.10)

$$
\Delta \boldsymbol{\kappa}=\boldsymbol{\kappa}^{(j+1)}-\boldsymbol{\kappa}^{(j)}=-\boldsymbol{V}^{(j)} \cdot\left[\alpha^{(j)} \boldsymbol{H}_{L M}^{(j)} \cdot \partial_{\boldsymbol{\kappa}} f\left(\boldsymbol{\kappa}^{(j)}\right)\right] .
$$

Conceptually speaking, the diagonal matrix $\boldsymbol{V}^{(j)}$ weights the step $\Delta \kappa_{1}$ for each parameter corresponding to the magnitude of $\kappa_{1}^{(j)}$, whereby

$$
\boldsymbol{V}^{(j)}=\operatorname{diag}\left(a_{1}^{(j)}, \ldots, a_{11}^{(j)}\right) .
$$


For the elaborated iteration process we choose $a_{11}=1000$ while all other weights we set to $a_{1}=1$.

\subsubsection{Verification}

Within the verification procedure the simulated data - calculated as based on the optimal parameters $\boldsymbol{\kappa}_{l m_{1}}^{l e v_{4}}$ for the FEM model with 2400 elements - is compared with the experimental data used for the parameter identification. By this the quality of the identified parameters and also the general ability of the constitutive model to describe the viscoelastic behavior of the material is analyzed. In particular, two representative identification nodes out of $N_{g}=116$ are chosen which are referred to by numbers 60 and 103 as shown in figure 7.31. The verification shows that the local minimization of the weighted least

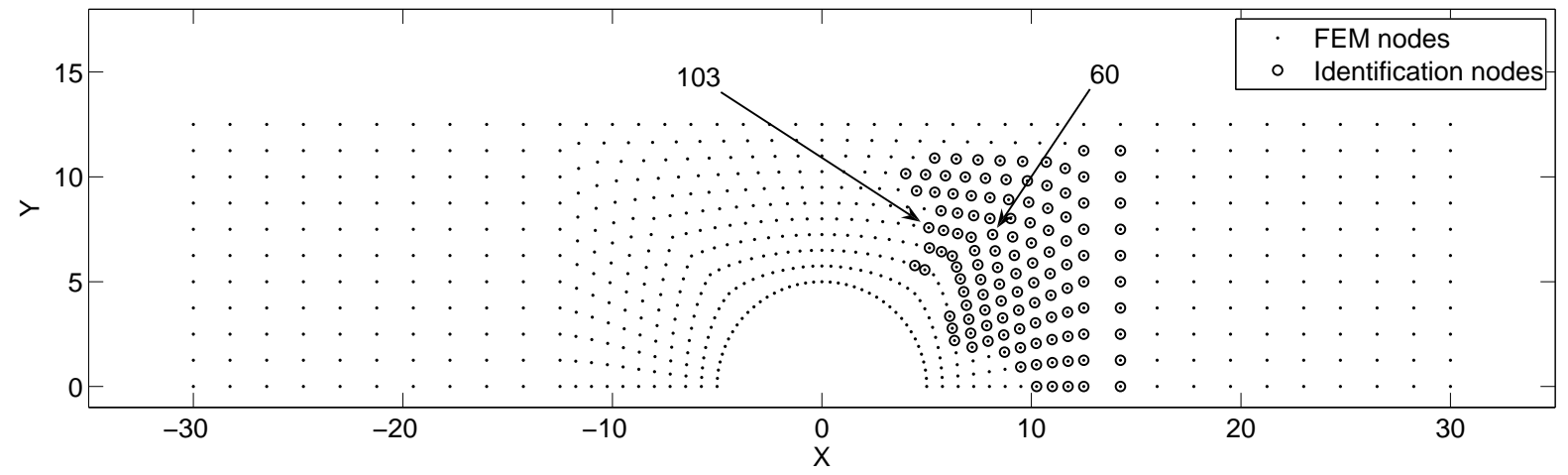

Figure 7.31: Identification nodes for the tests $A, B$, and $C$ in the FEM model with 2400 elements; representative identification nodes 60 and 103 .

squares functional delivers a parameter set which enables to fit the simulations to the respective experiments for all three tests in an average (weighted) way. Generally for the tests $A, B$ and $C$ a good qualitative and quantitative agreement between the experiment and the simulation can be observed (see figures $7.32-7.37$ ). 

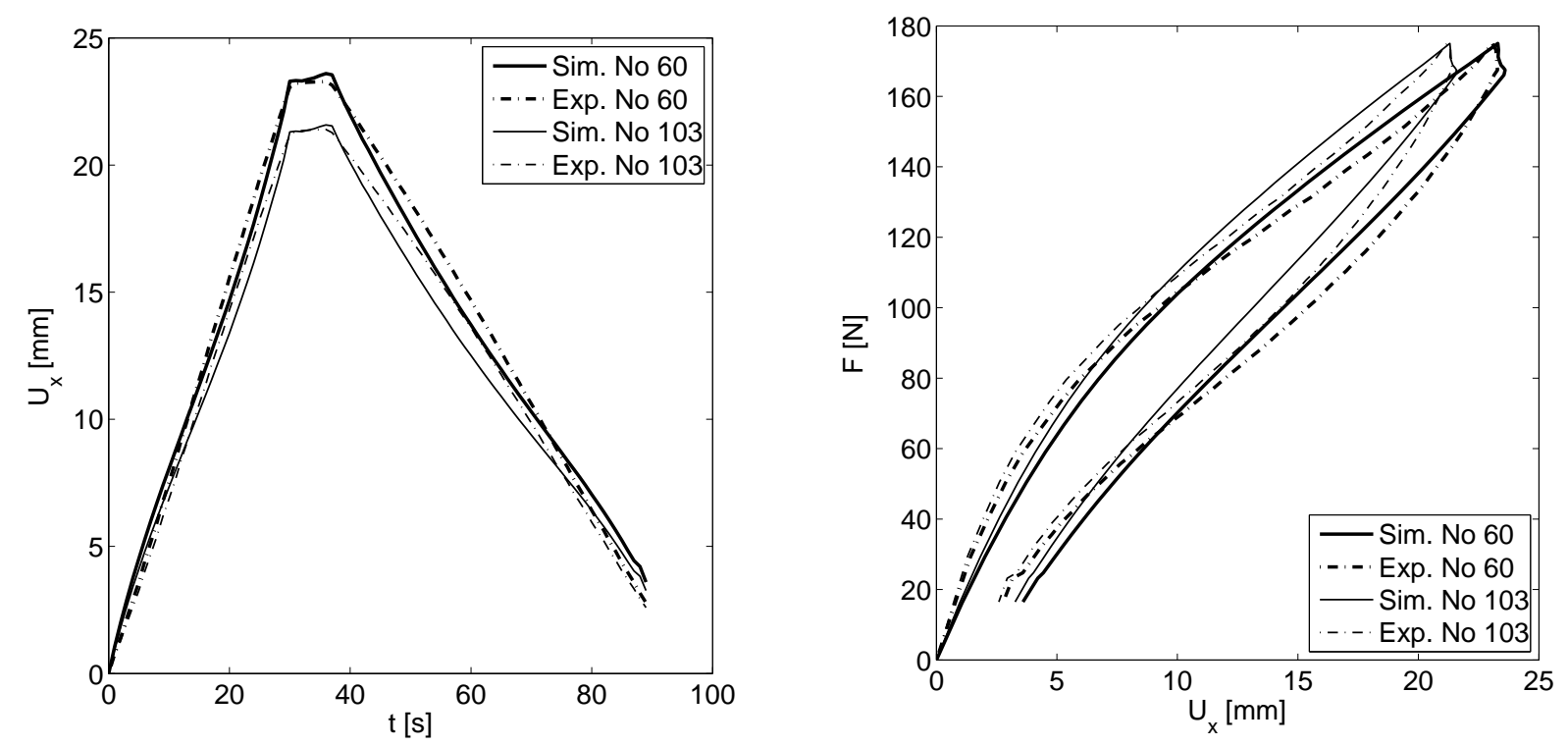

Figure 7.32: Verification for test $A$; displacements in longitudinal direction.
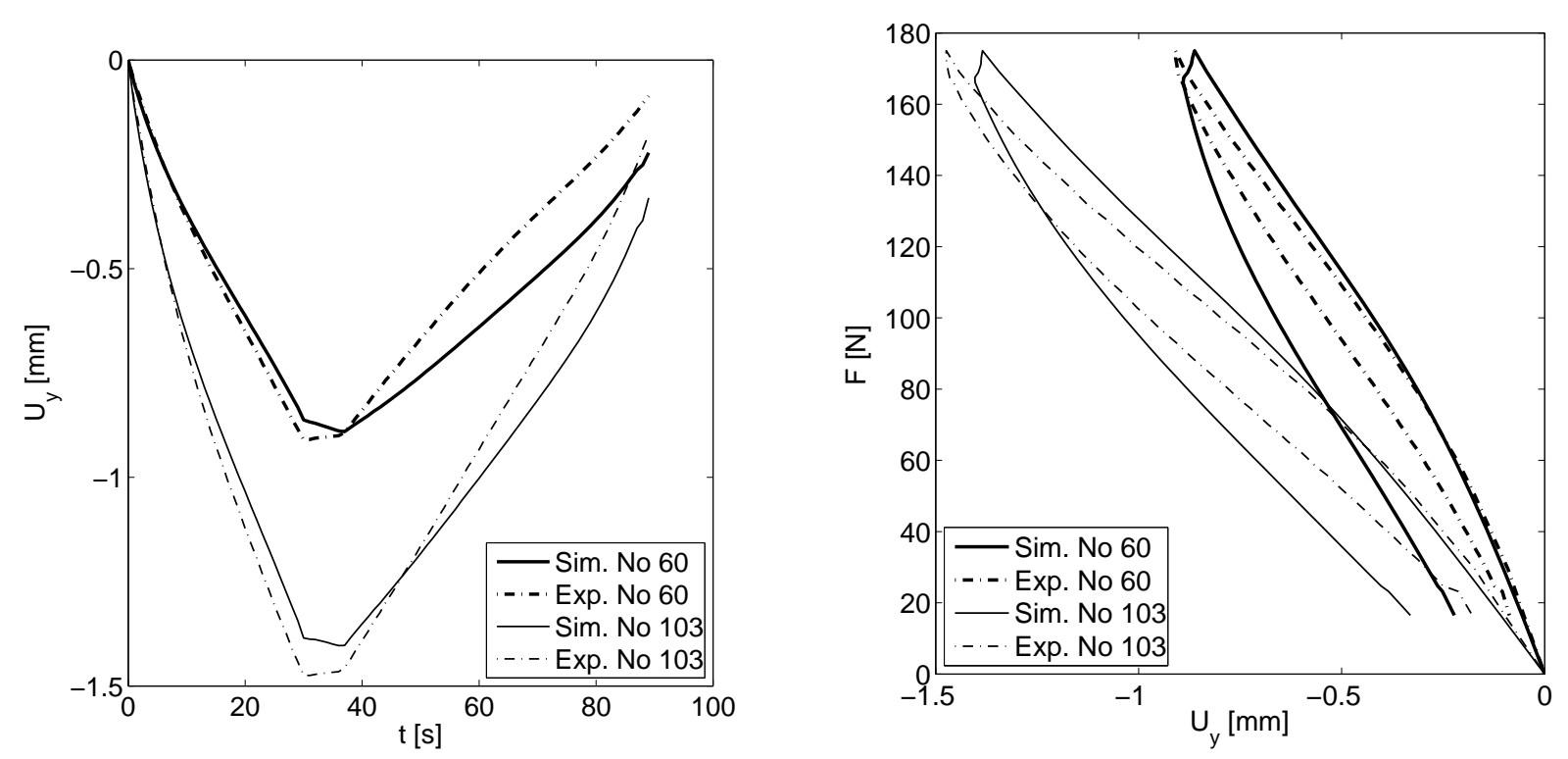

Figure 7.33: Verification for test $A$; displacements in transverse direction. 

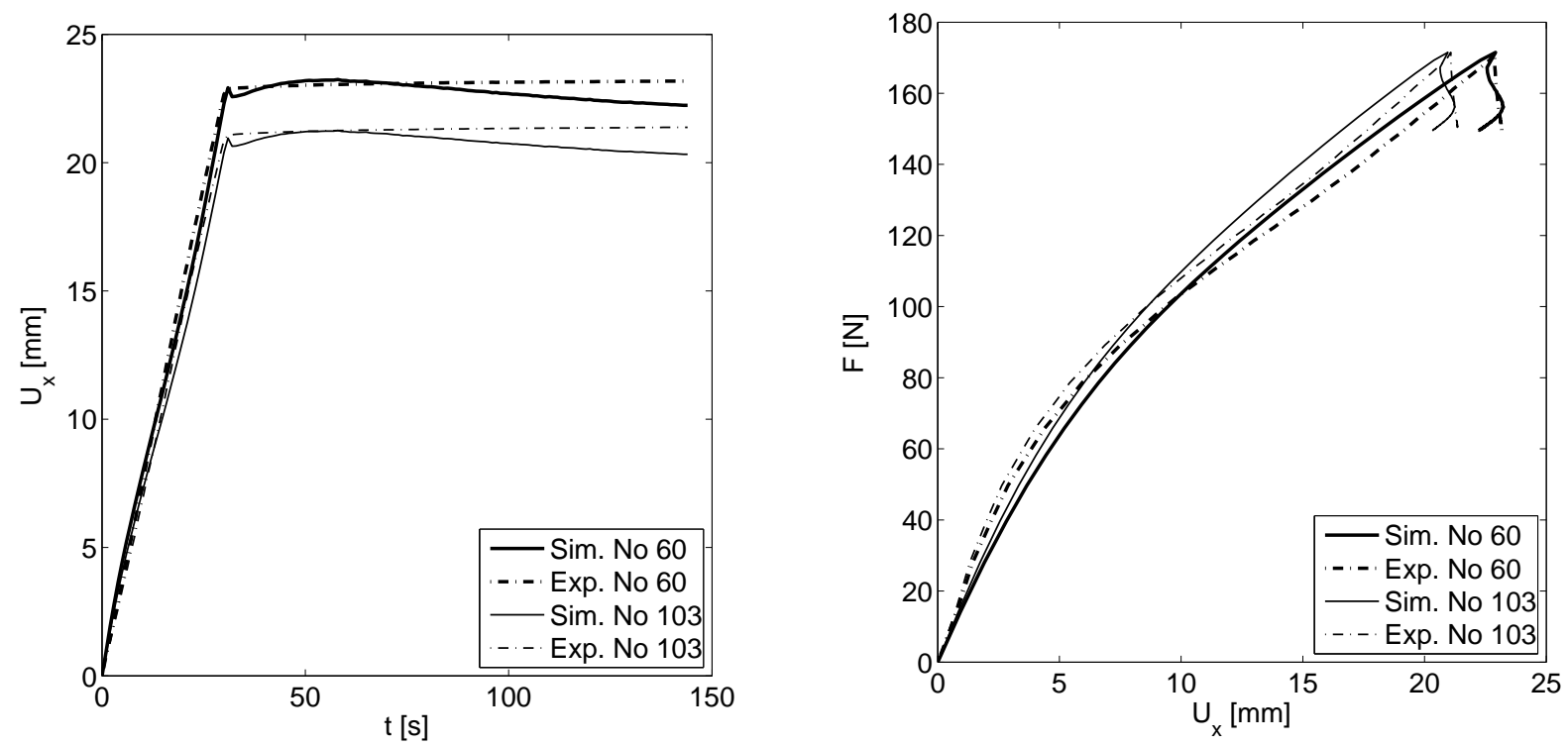

Figure 7.34: Verification for test $B$; displacements in longitudinal direction.
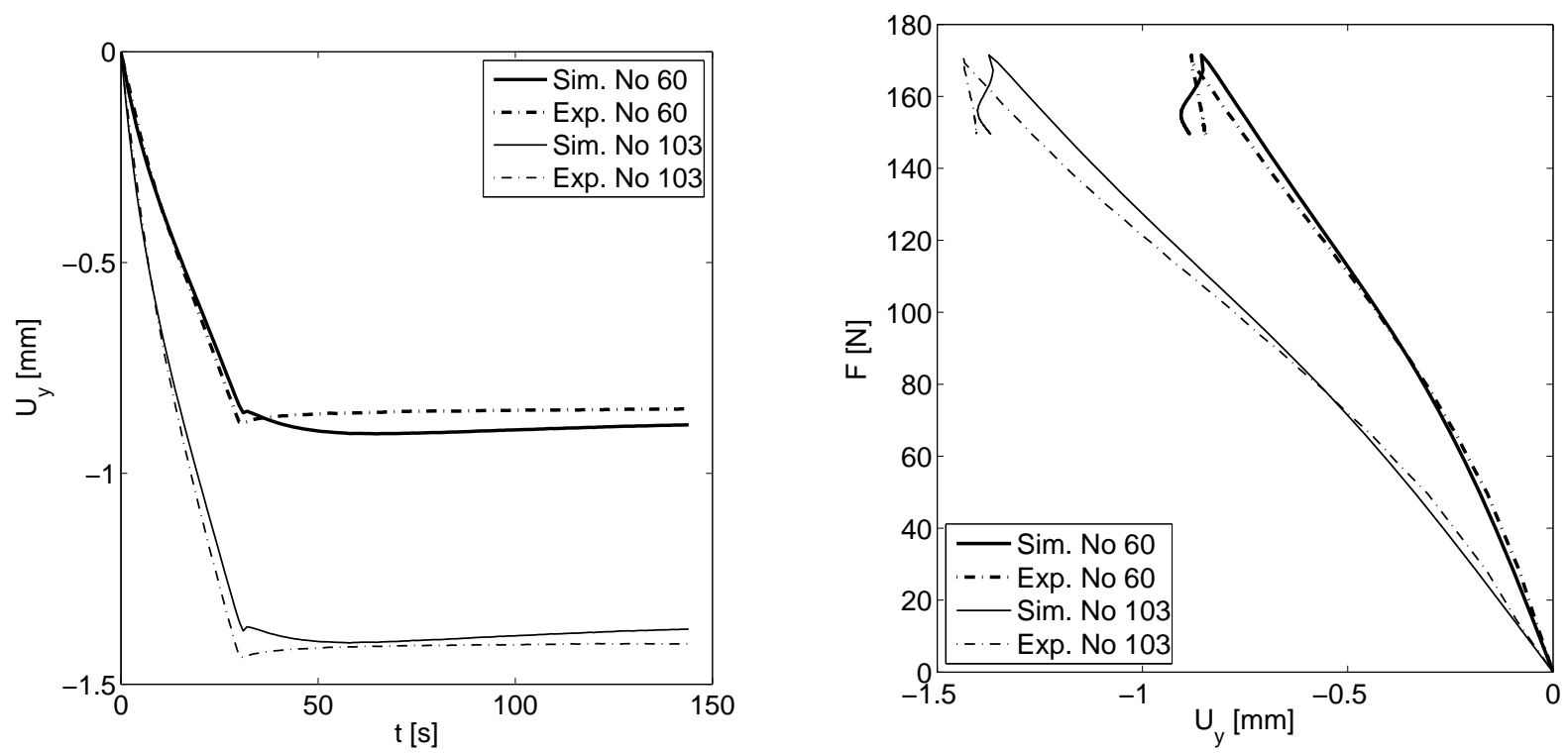

Figure 7.35: Verification for test $B$; displacements in transverse direction.

The tests $A$ (time $\left.t_{A}\right)$ and $B$ (time $t_{B}$ ) reflect the short time viscoelastic behavior. For the respective load application $\left(0 s \leq t_{A} \leq 30 s\right.$ and $\left.0 s \leq t_{B} \leq 31 s\right)$ and the holding times in the time segments $30 s \leq t_{A} \leq 36 \mathrm{~s}$ and $31 \mathrm{~s} \leq t_{B} \leq 37 \mathrm{~s}$ the simulations deliver a very good agreement to the experimental data. The relaxation time $\kappa_{\text {neq }}^{\mathrm{k}=1}$ is predominantly activated by the short time tests $A$ and $B$, and it follows that the identification of the parameters of the first relaxation term are identified to fit the short time viscoelastic behavior of $A$ and $B$ in an average sense. As for $36 s \leq t_{A} \leq 89 \mathrm{~s}$ and $37 s \leq t_{B} \leq 113 \mathrm{~s}$ the characteristics of the tests $A$ and $B$ are no longer identical, this averaging becomes obvious for these time segments. 

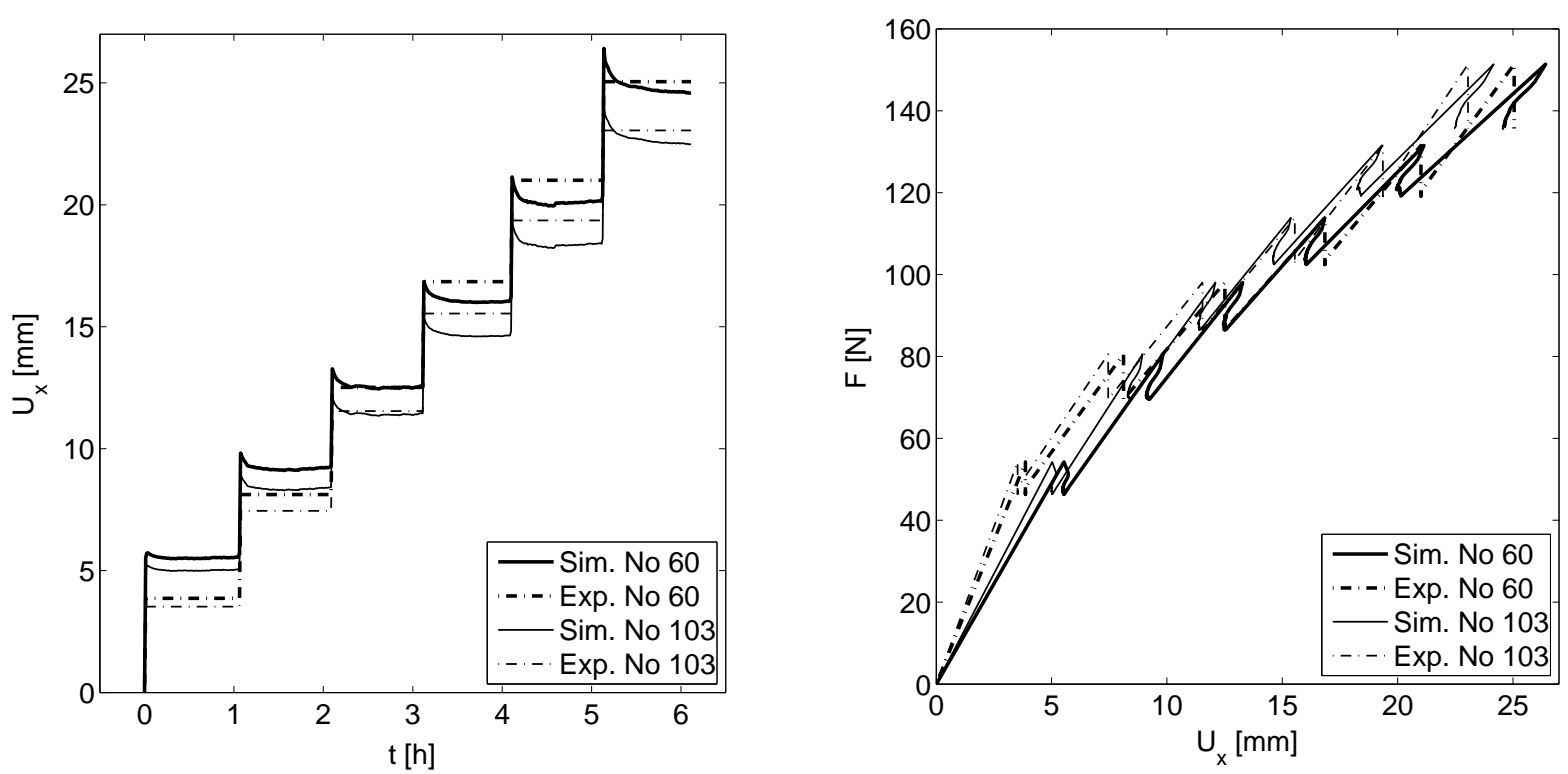

Figure 7.36: Verification for test $C$; displacements in longitudinal direction.
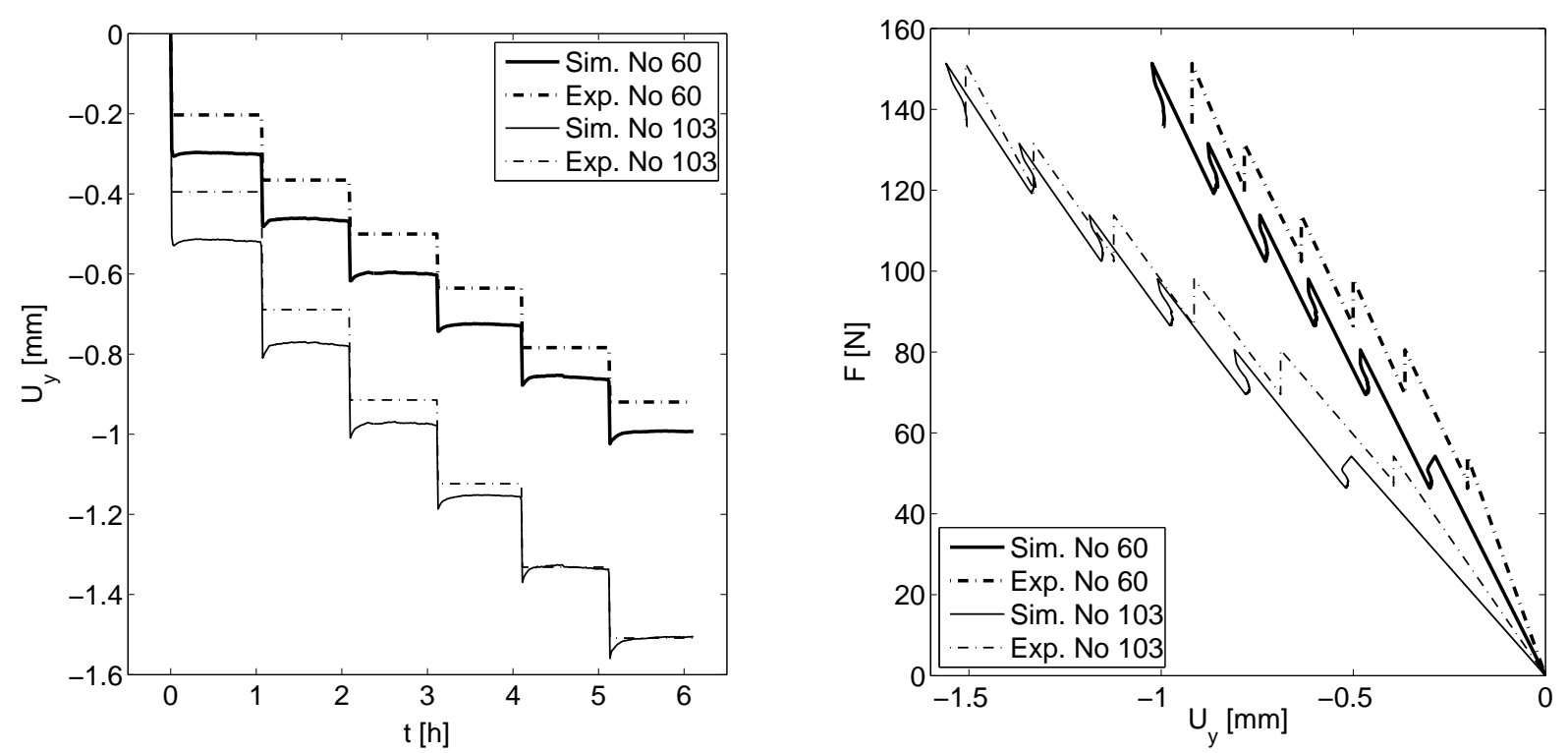

Figure 7.37: Verification for test $C$; displacements in transverse direction.

Test $C$ reflects the long term viscoelastic response of the material for the six loading steps considered. Therefore the contribution of test $C$ within the least squares algorithm mainly determines the identification of the second relaxation time $\hat{\tau}^{\mathrm{k}=2}$. As mentioned in subsection 7.3.5, $\hat{\tau}^{\mathrm{k}=2}$ correlates strongly with the parameters for the deviatoric part of the equilibrium part of the free energy. On the one hand this is due to the difference in size of the identified parameters $\hat{\tau}^{\mathrm{k}=2}=9806.90 \mathrm{~s}$ compared with the first relaxation time $\hat{\tau}^{\mathrm{k}=1}=4.55 \mathrm{~s}$. For the simulation of the tests $A$ and $B$ the second relaxation term almost behaves like an equilibrium term. On the other hand figure 7.36 shows that $U_{x}$ versus the time $t$ has to be averaged for the six loading steps which makes it difficult to identify a stable parameter $\hat{\tau}^{\mathrm{k}=2}$. 
Figures 7.38, 7.39 and 7.40 show the euclidean norm of the equilibrium- and nonequilibrium parts of the Cauchy stresses as well as for the total Cauchy stresses for the identification node 60 in the tests $A, B$ and $C$.

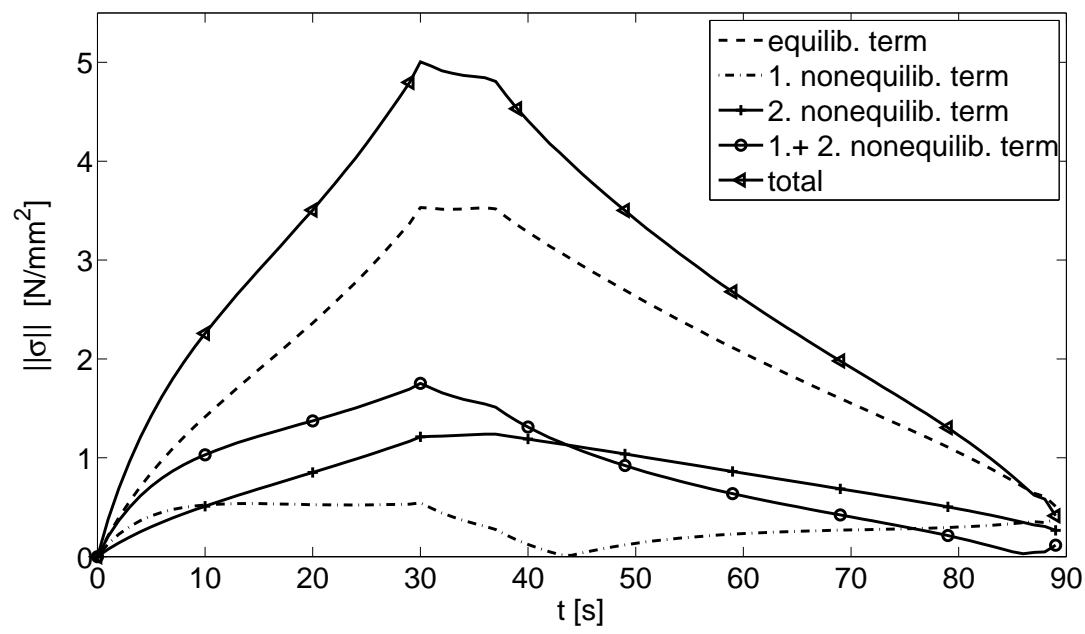

Figure 7.38: Test $A$ : Euclidean norm of the equilibrium- and non-equilibrium parts of the Cauchy stresses as well as for the total Cauchy stresses; Identification node 60.

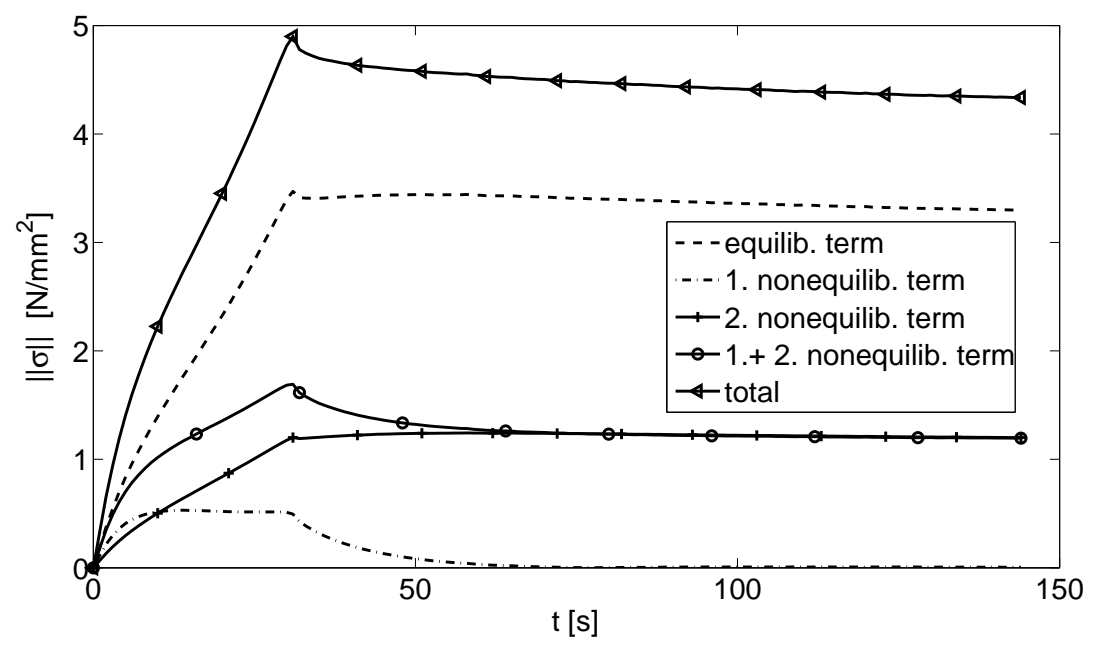

Figure 7.39: Test $B$ : Euclidean norm of the equilibrium- and non-equilibrium parts of the Cauchy stresses as well as for the total Cauchy stresses; Identification node 60 .

These curves can also serve as an indicator for the ability of the constitutive model combined with the identified parameters to simulate the viscoelastic behavior of the material tested. For the loading steps in the tests $A, B$ and $C$ the equilibrium stress is supposed to stay approximately constant whereby the reduction of the forces (measured in the experiments and applied as force loads in the simulation) is approximately satisfied by the relaxation of the non-equilibrium stresses. For the tests $A$ and $B$ this requirement is approximately given. For the six different loading steps in test $C$ the viscous stress relaxes in a way to lead the equilibrium terms to an almost horizontal line (at the beginning of each loading interval, however, peak levels are apparently observed). 


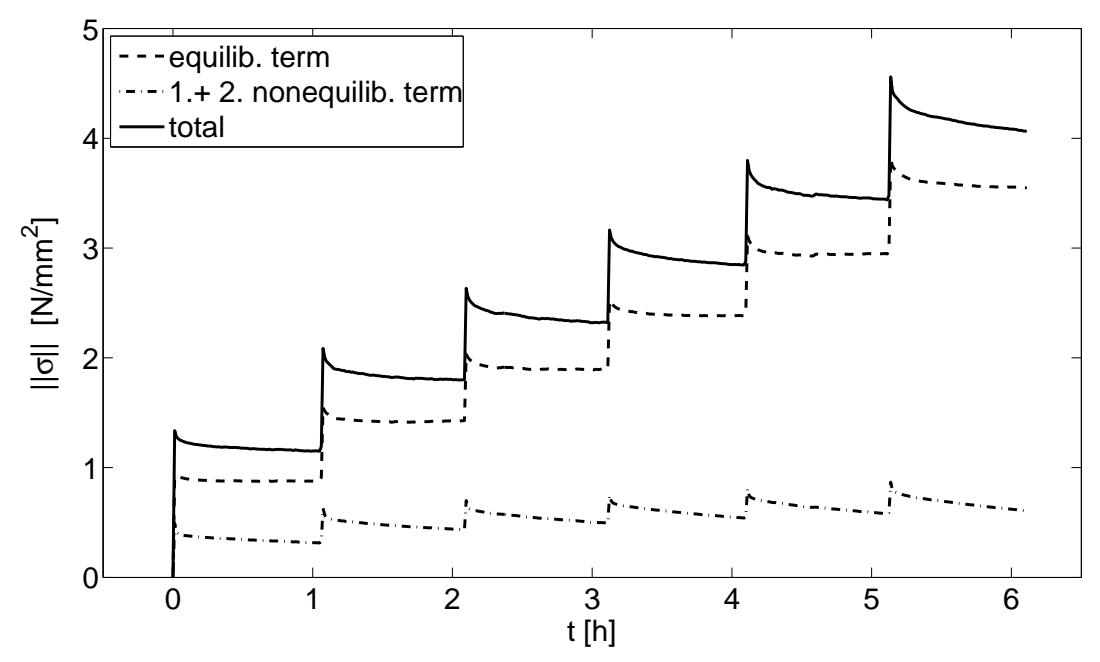

Figure 7.40: Test $C$ : Euclidean norm of the equilibrium- and non-equilibrium parts of the Cauchy stresses as well as for the total Cauchy stresses; Identification node 60.

Note that in both tests $A$ and $B$ the equilibrium state for the first relaxation term is reached. In test $A$ after the load reduction for $t_{A}>36 s$ the dynamic equilibrium state is reached at $t_{A} \approx 43 \mathrm{~s}$. In contrast test $B$ reaches the thermodynamic equilibrium state at $t_{B} \approx 75 \mathrm{~s}$.

Figures 7.41-7.44 show the total-, equilibrium- and non-equilibrium parts of the Cauchy stress in $x$-direction versus the component of the left Cauchy-Green tensor in normal direction $b_{11}$ for the identification node 60 in the test $A$.

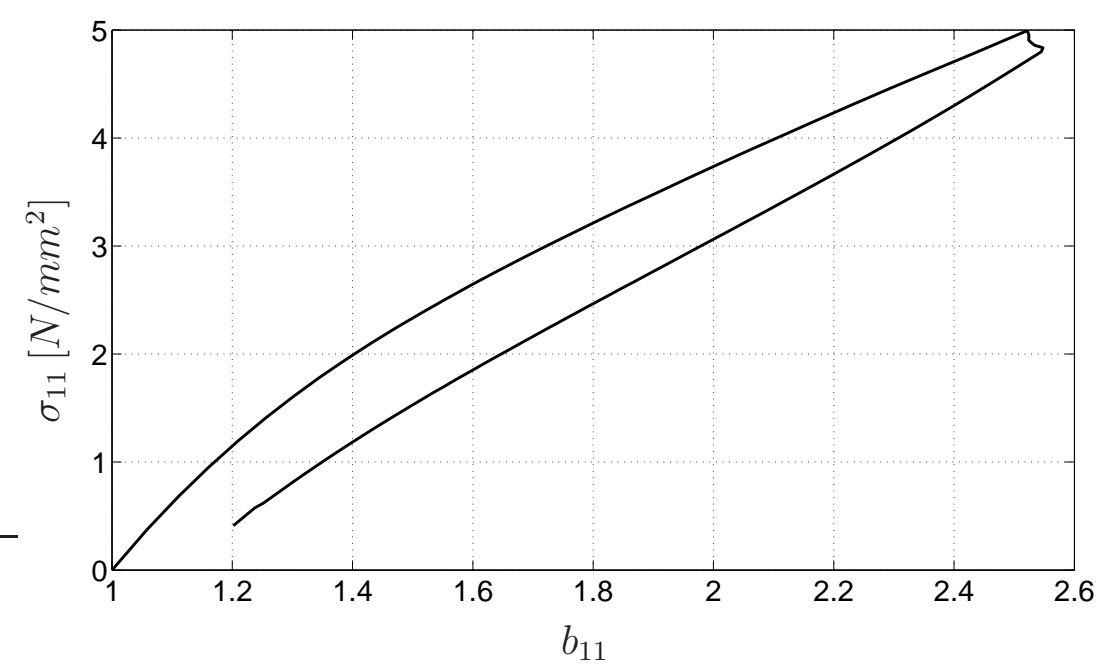

Figure 7.41: Test $A$ : Total Cauchy stress in x-direction $\sigma_{11}$ versus the component of the left CauchyGreen tensor in normal direction $b_{11}$; Identification node 60 . 


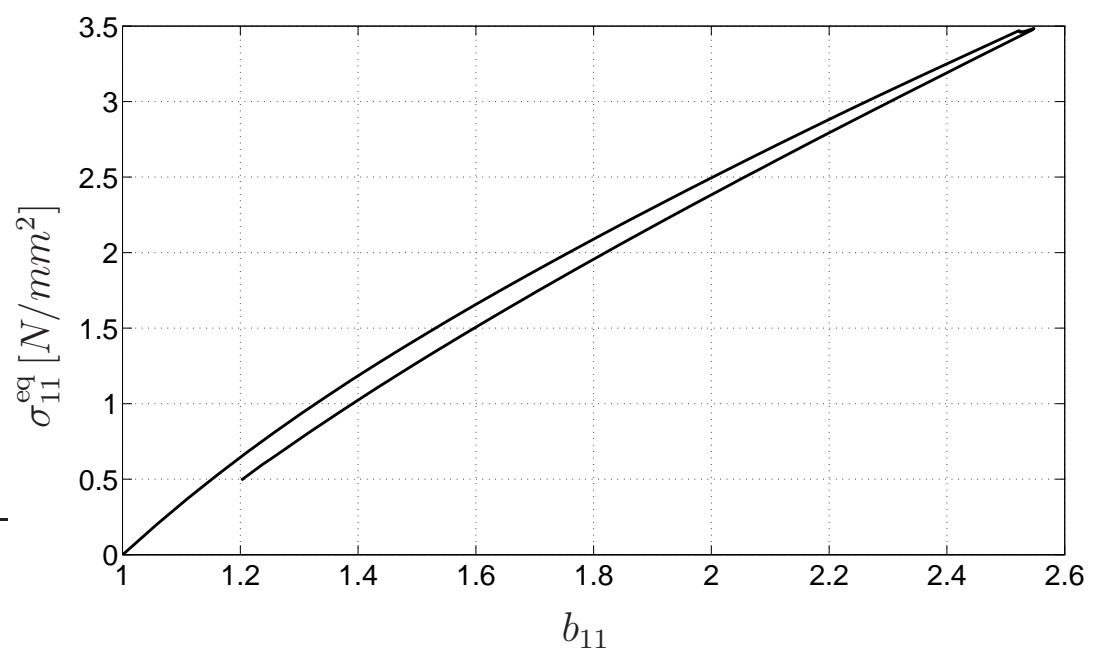

Figure 7.42: Test $A$ : Equilibrium Cauchy stress in $\mathrm{x}$-direction $\sigma_{11}^{\mathrm{eq}}$ versus the component of the left Cauchy-Green tensor in normal direction $b_{11}$; Identification node 60.

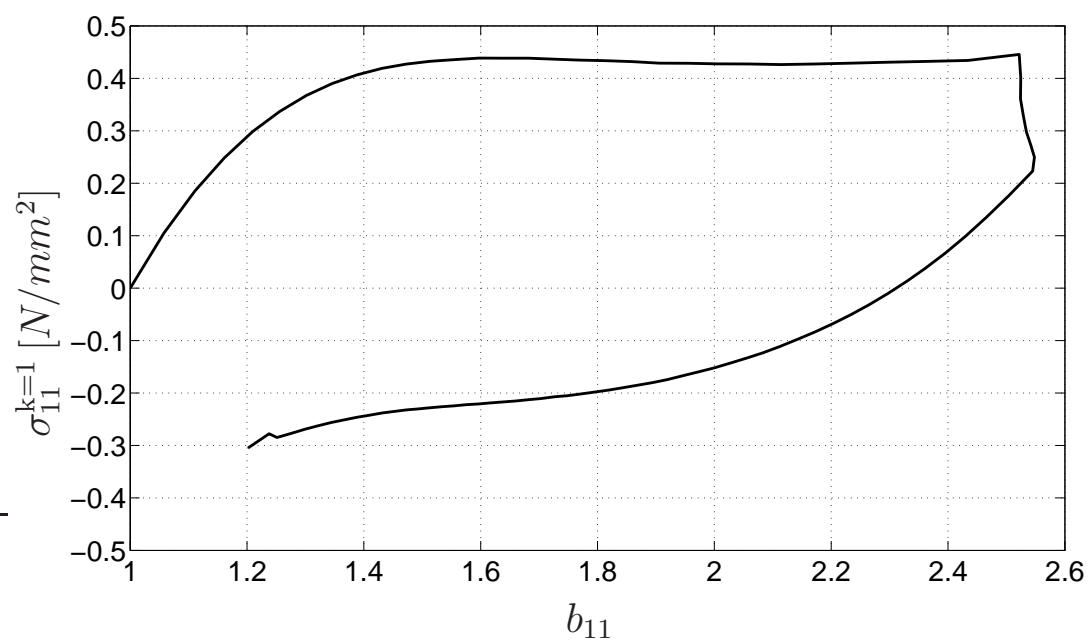

Figure 7.43: Test $A$ : Non-equilibrium Cauchy stress for the first relaxation module in $\mathrm{x}$-direction $\sigma_{11}^{\mathrm{k}=1}$ versus the component of the left Cauchy-Green tensor in normal direction $b_{11}$; Identification node 60 . 


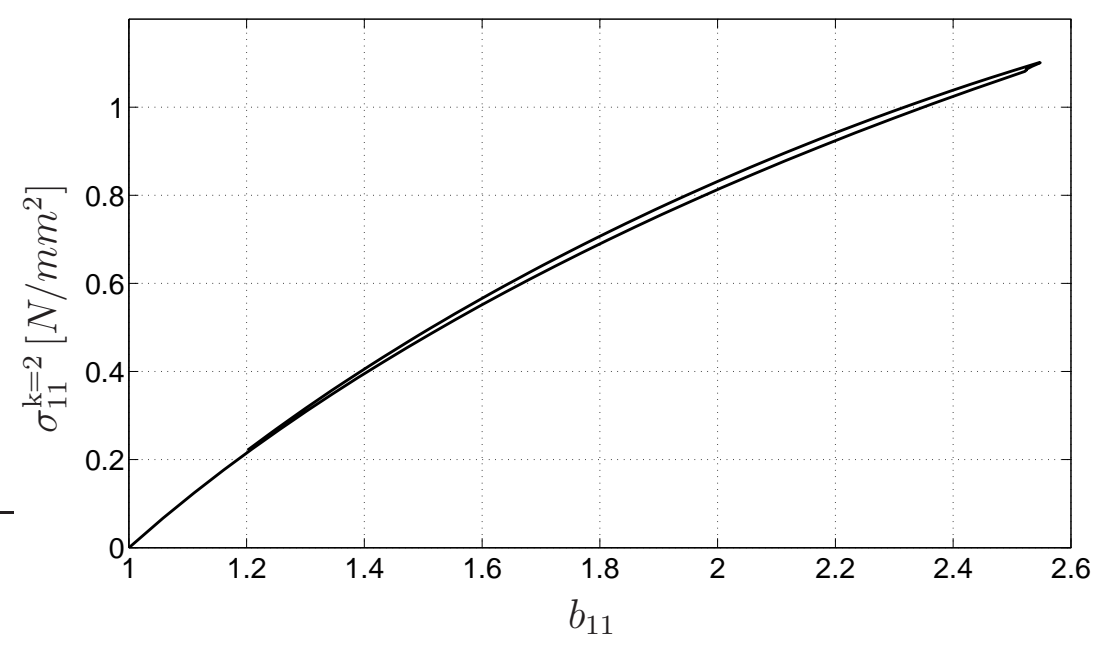

Figure 7.44: Test $A$ : Non-equilibrium Cauchy stress for the second relaxation module in $\mathrm{x}$-direction $\sigma_{11}^{\mathrm{k}=2}$ versus the component of the left Cauchy-Green tensor in normal direction $b_{11}$; Identification node 60 .

Figures 7.45-7.48 show the total-, equilibrium- and non-equilibrium parts of the Cauchy stress in $x$-direction versus the component of the left Cauchy-Green tensor in normal direction $b_{11}$ for the identification node 60 in the test $B$.

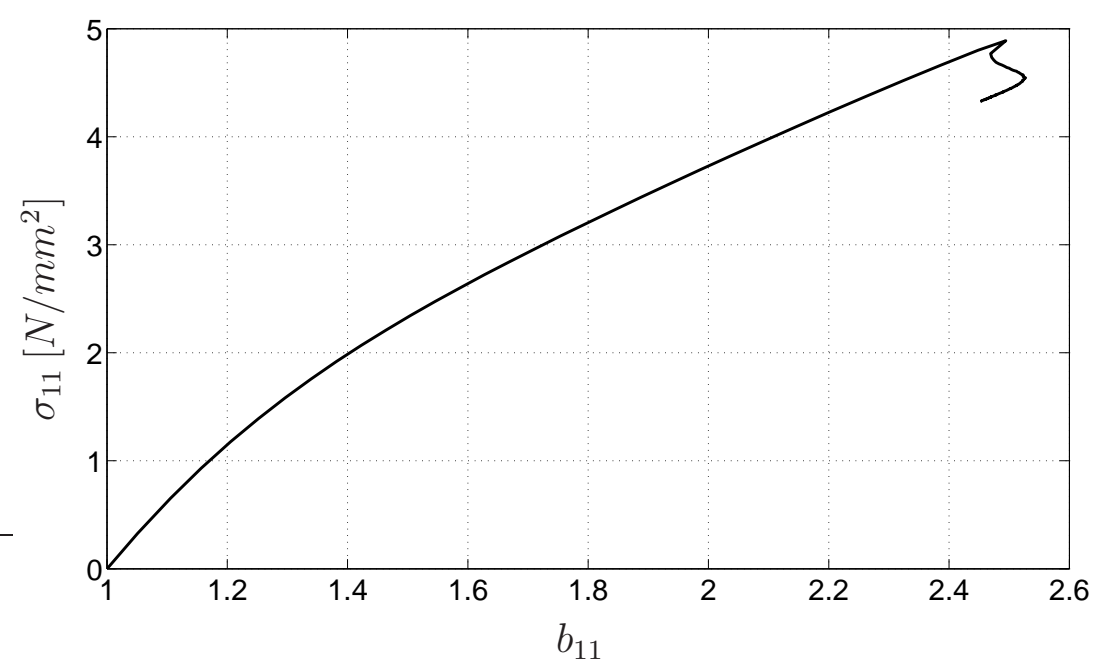

Figure 7.45: Test $B$ : Total Cauchy stress in $\mathrm{x}$-direction $\sigma_{11}$ versus the component of the left CauchyGreen tensor in normal direction $b_{11}$; Identification node 60 . 


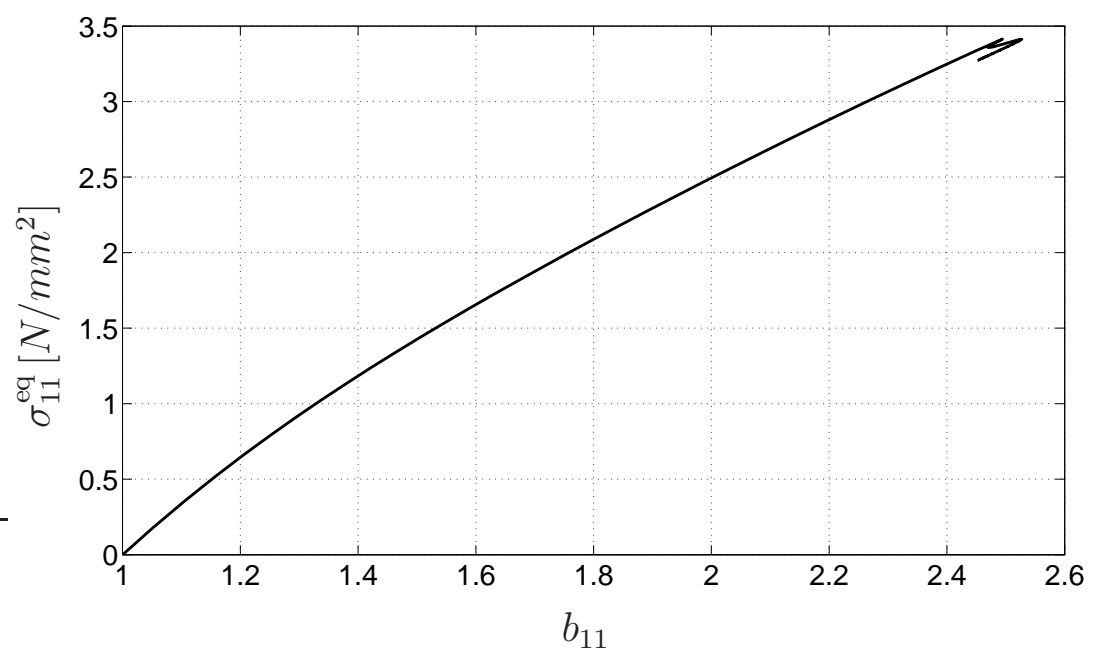

Figure 7.46: Test $B$ : Equilibrium Cauchy stress in $\mathrm{x}$-direction $\sigma_{11}^{\mathrm{eq}}$ versus the component of the left Cauchy-Green tensor in normal direction $b_{11}$; Identification node 60.

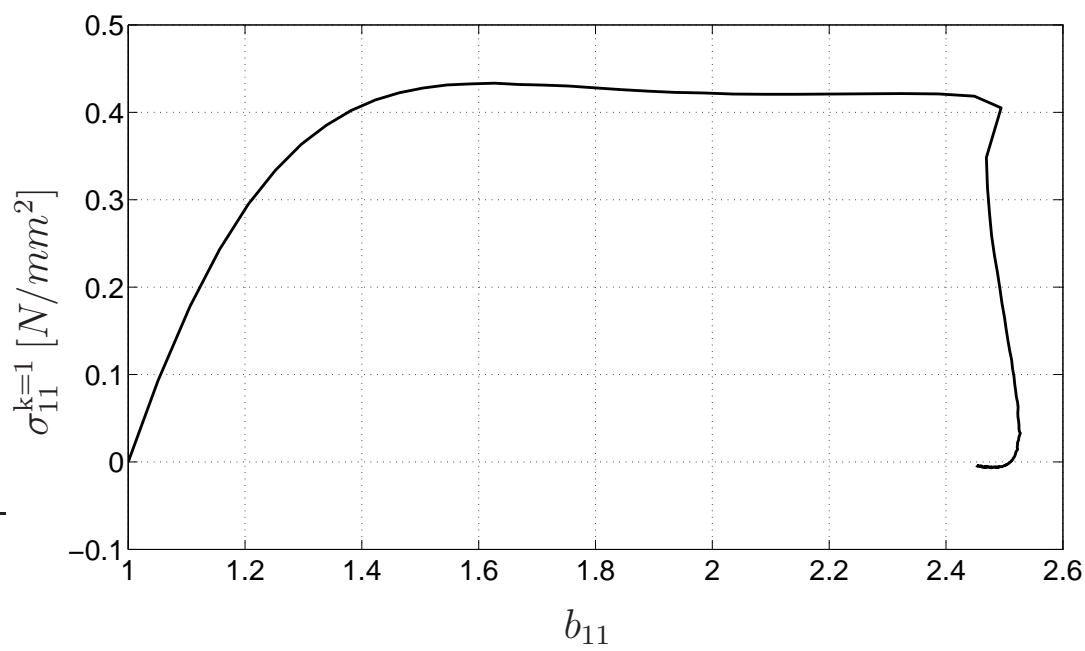

Figure 7.47: Test $B$ : Non-equilibrium Cauchy stress for the first relaxation module in $\mathrm{x}$-direction $\sigma_{11}^{\mathrm{k}=1}$ versus the component of the left Cauchy-Green tensor in normal direction $b_{11}$; Identification node 60 . 


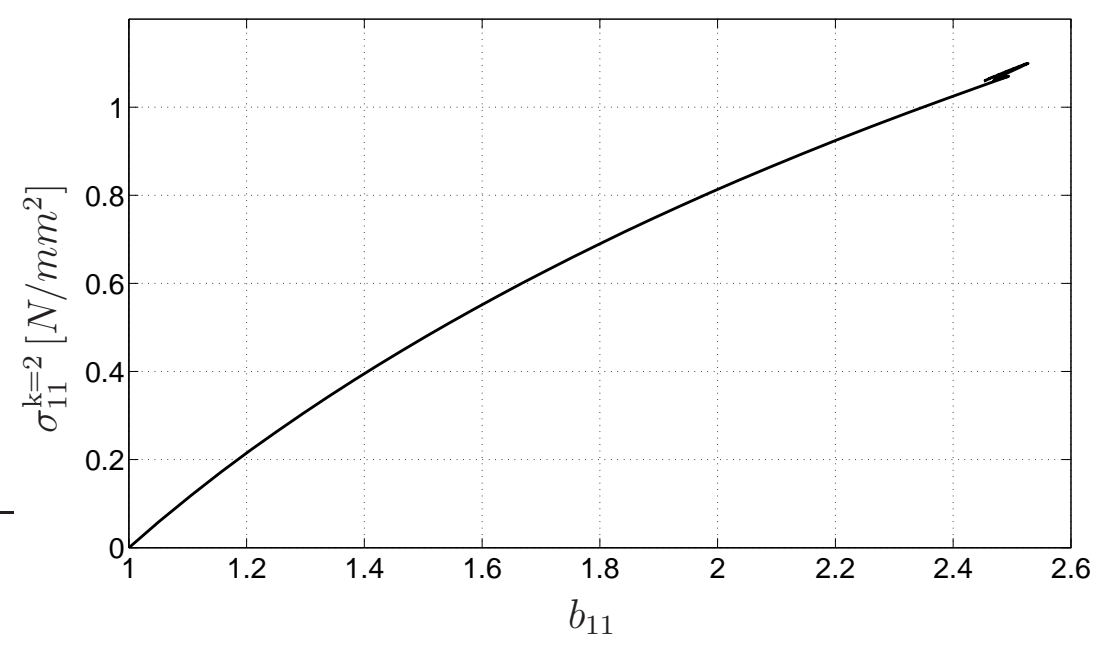

Figure 7.48: Test $B$ : Non-equilibrium Cauchy stress for the second relaxation module in $\mathrm{x}$-direction $\sigma_{11}^{\mathrm{k}=2}$ versus the component of the left Cauchy-Green tensor in normal direction $b_{11}$; Identification node 60 .

Figures 7.49-7.52 show the total-, equilibrium- and non-equilibrium parts of the Cauchy stress in $x$-direction versus the component of the left Cauchy-Green tensor in normal direction $b_{11}$ for the identification node 60 in the test $C$.

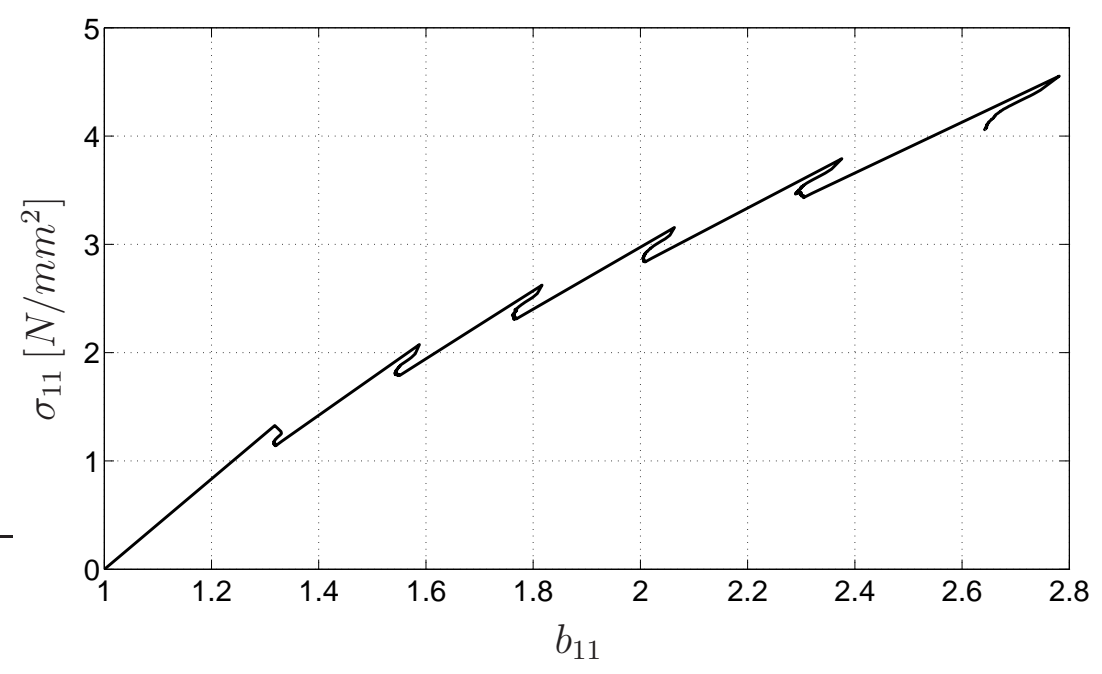

Figure 7.49: Test $C$ : Total Cauchy stress in $\mathrm{x}$-direction $\sigma_{11}$ versus the component of the left CauchyGreen tensor in normal direction $b_{11}$; Identification node 60 . 


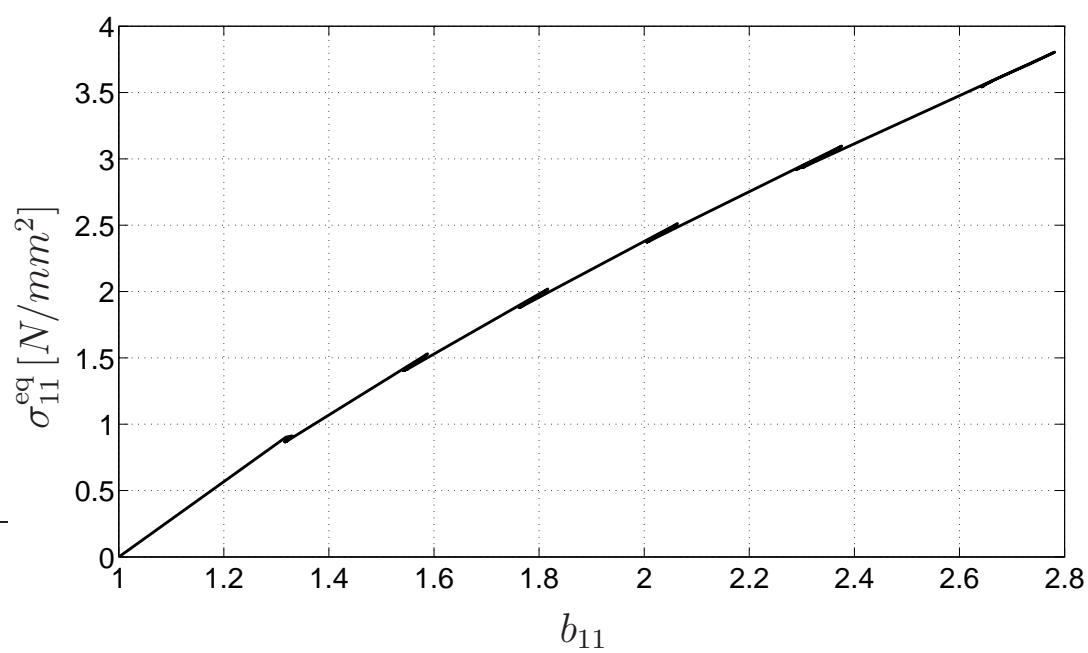

Figure 7.50: Test $C$ : Equilibrium Cauchy stress in $\mathrm{x}$-direction $\sigma_{11}^{\mathrm{eq}}$ versus the component of the left Cauchy-Green tensor in normal direction $b_{11}$; Identification node 60.

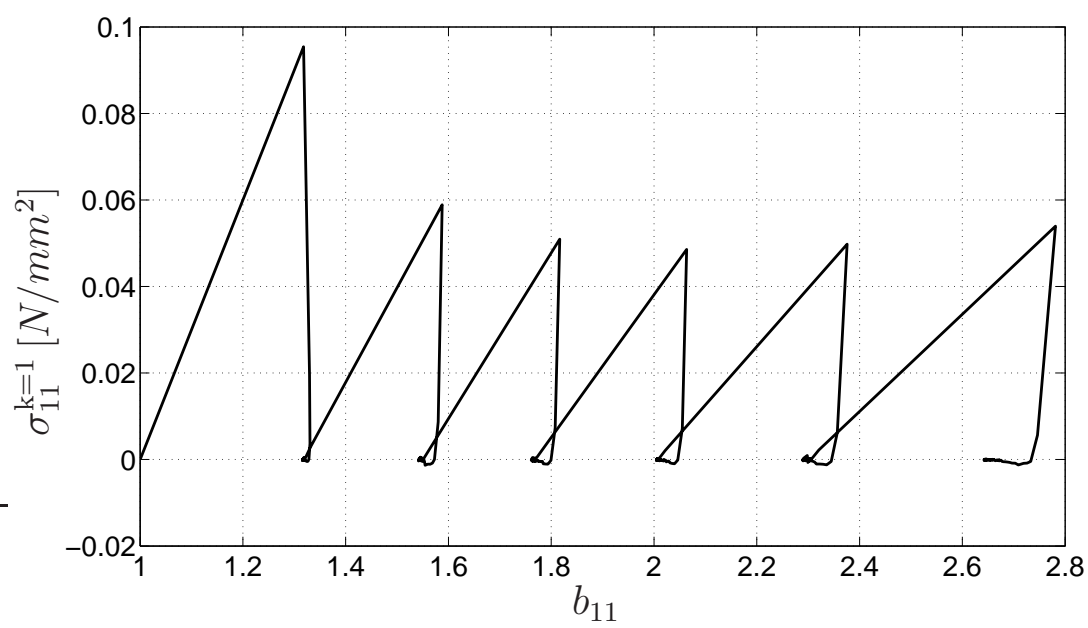

Figure 7.51: Test $C$ : Non-equilibrium Cauchy stress for the first relaxation module in $\mathrm{x}$-direction $\sigma_{11}^{\mathrm{k}=1}$ versus the component of the left Cauchy-Green tensor in normal direction $b_{11}$; Identification node 60 . 


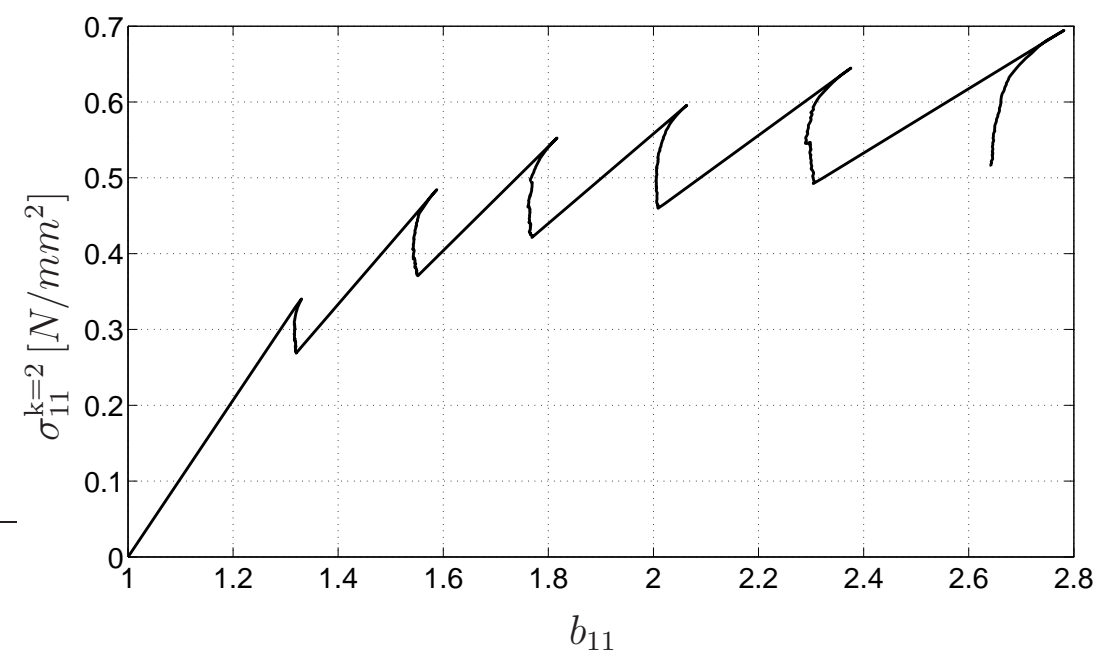

Figure 7.52: Test $C$ : Non-equilibrium Cauchy stress for the second relaxation module in $\mathrm{x}$-direction $\sigma_{11}^{\mathrm{k}=2}$ versus the component of the left Cauchy-Green tensor in normal direction $b_{11}$; Identification node 60 .

\subsubsection{Extended verification}

In view of a simplified identification process we did not make use of all available experimental data for the tests $A, B$, and $C$. Thus the remaining displacement data can be used for an extended verification. Figure 7.53 shows the entire FEM model (9600 elements) with the discretization of the corresponding FEM model based on 2400 elements which serves for the identification routine. The nodes for the extended verification for the different tests are marked $(V 1-V 6)$. This extended verification shows that also the

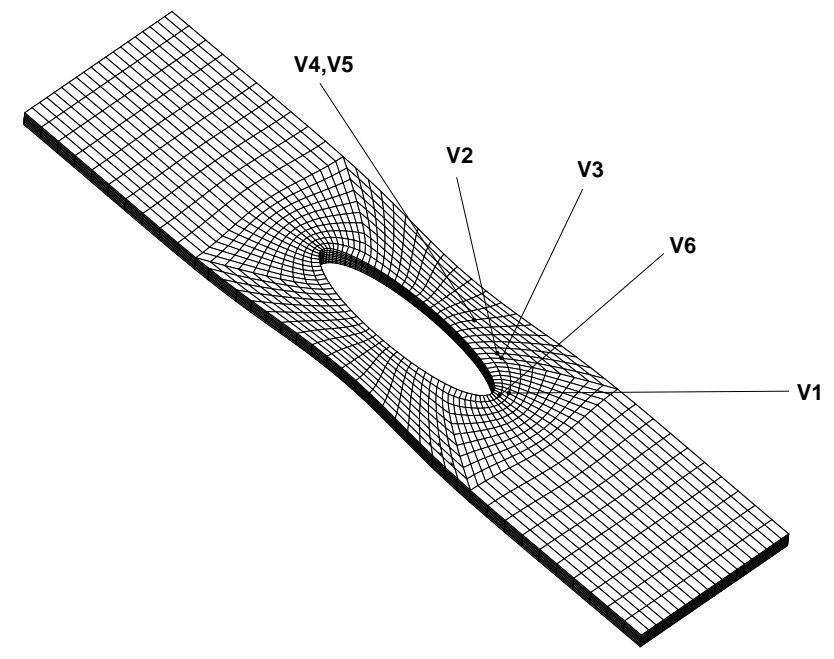

Figure 7.53: End of test $C$; nodes for the extended verification.

experimental data which is not used for identification, is in good agreement with the corresponding simulated data which is reflected by the results shown in figures 7.54-7.59, wherein displacement versus time curves are highlighted by analogy with previous figures. 

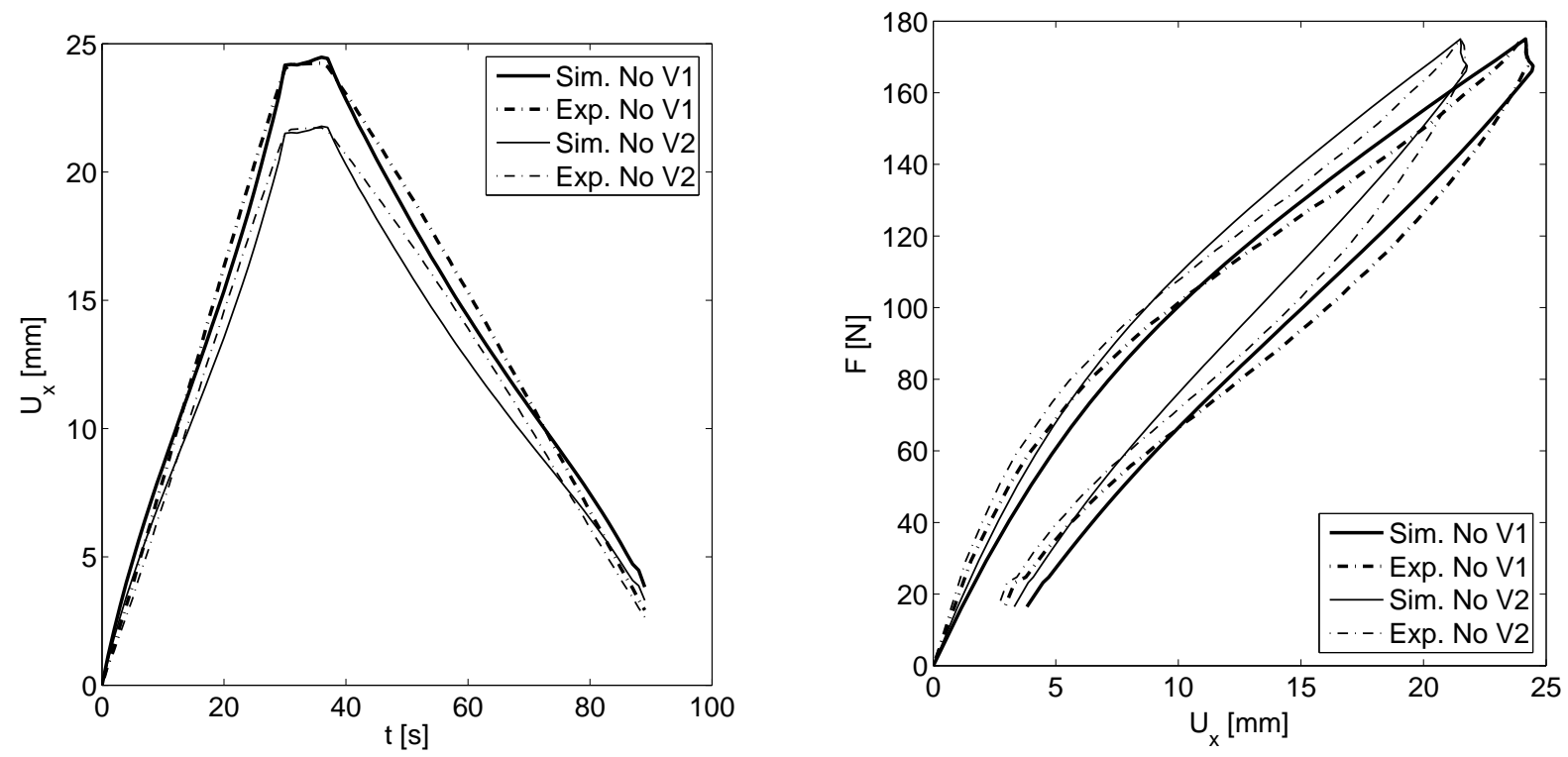

Figure 7.54: Extended verification for test $A$; displacements in longitudinal direction.
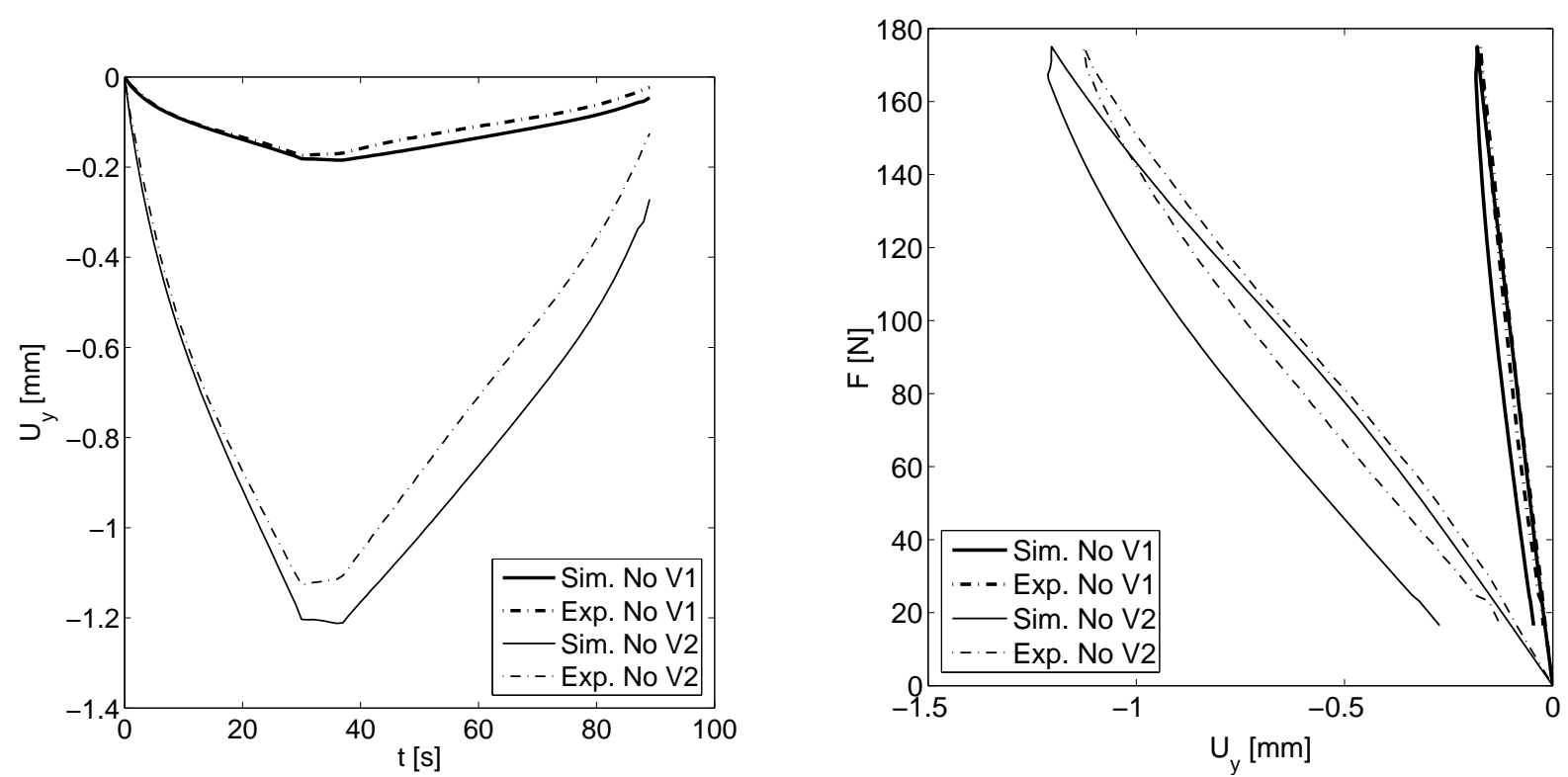

Figure 7.55: Extended verification for test $A$; displacements in transverse direction. 

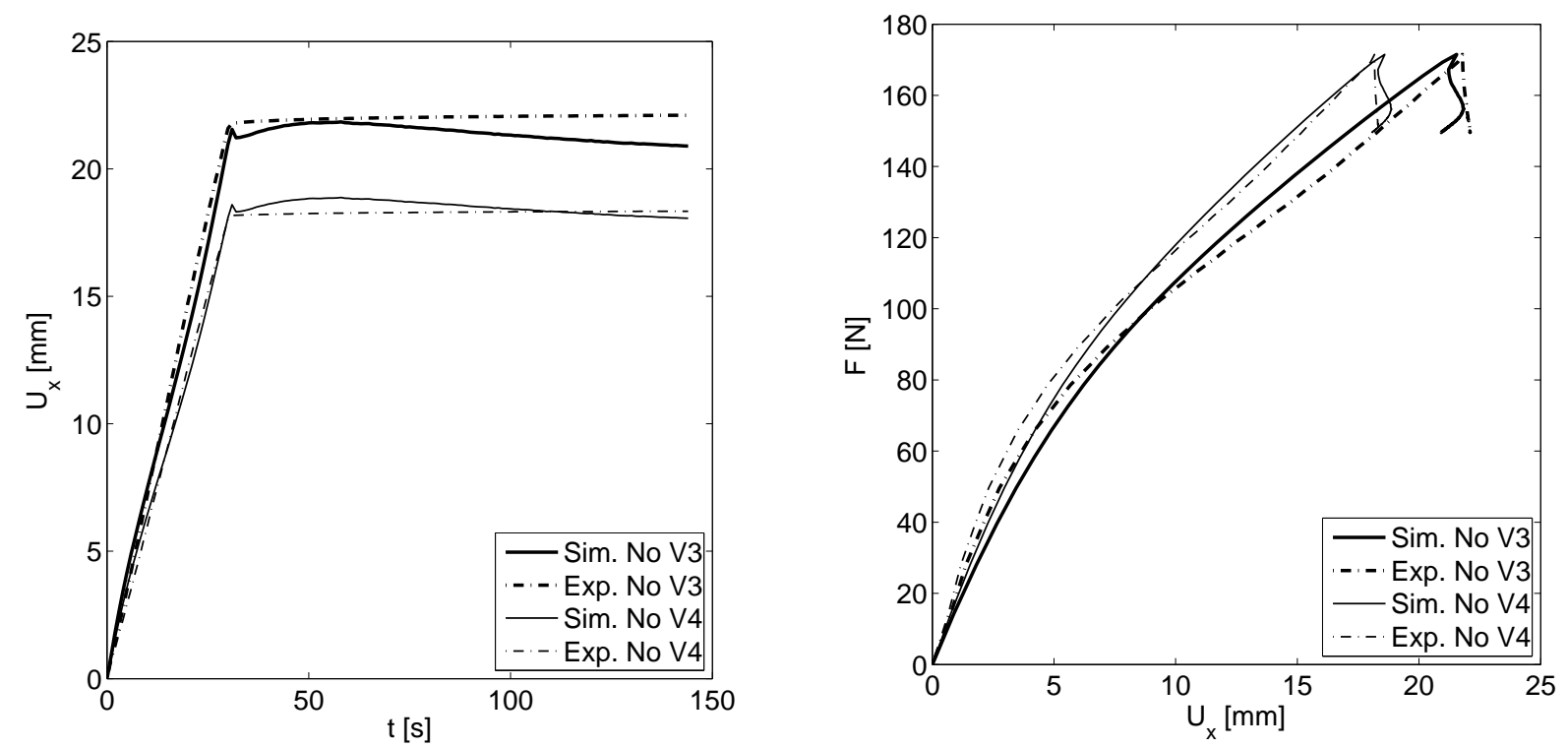

Figure 7.56: Extended verification for test $B$; displacements in longitudinal direction.
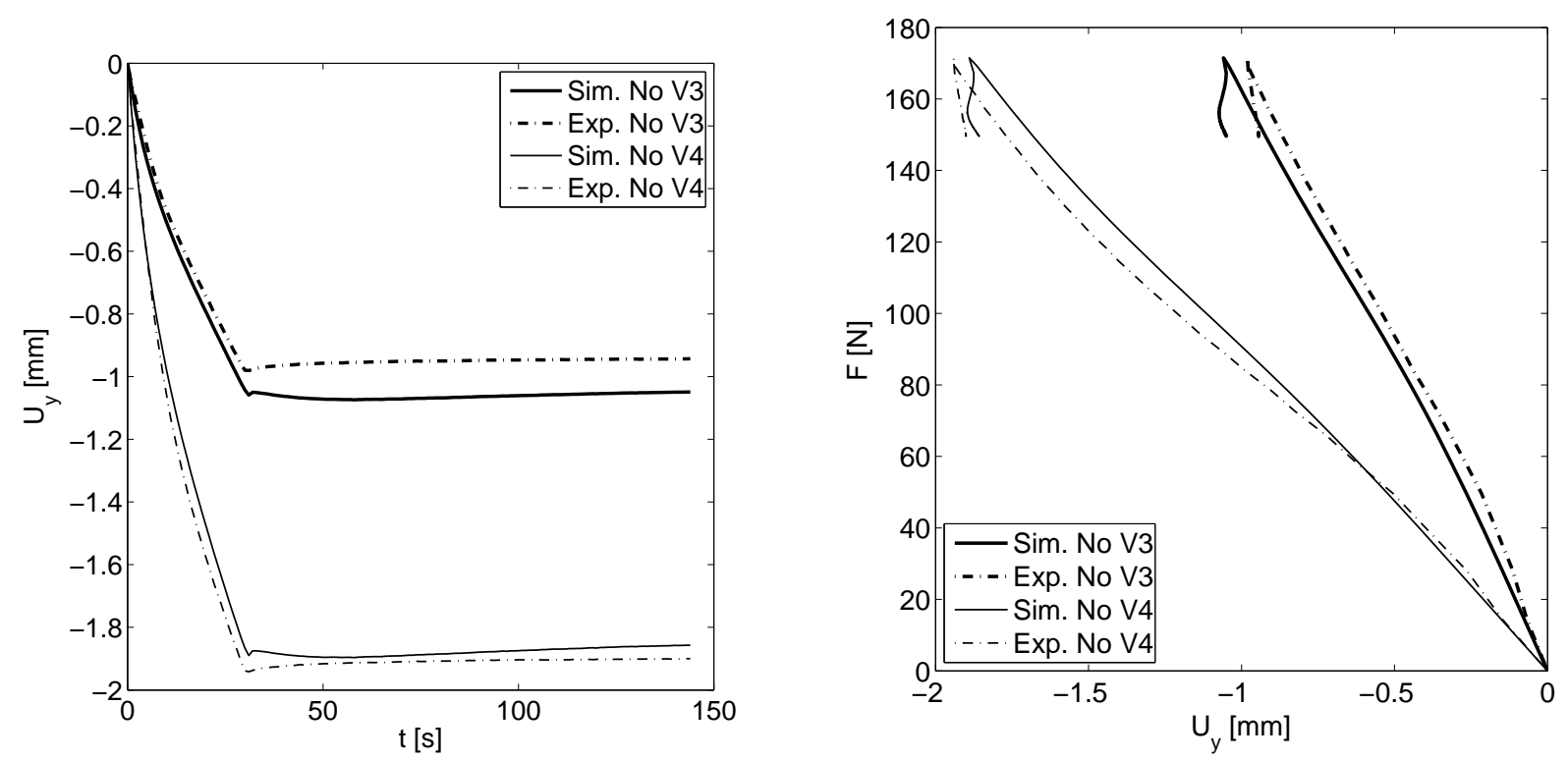

Figure 7.57: Extended verification for test $B$; displacements in transverse direction. 

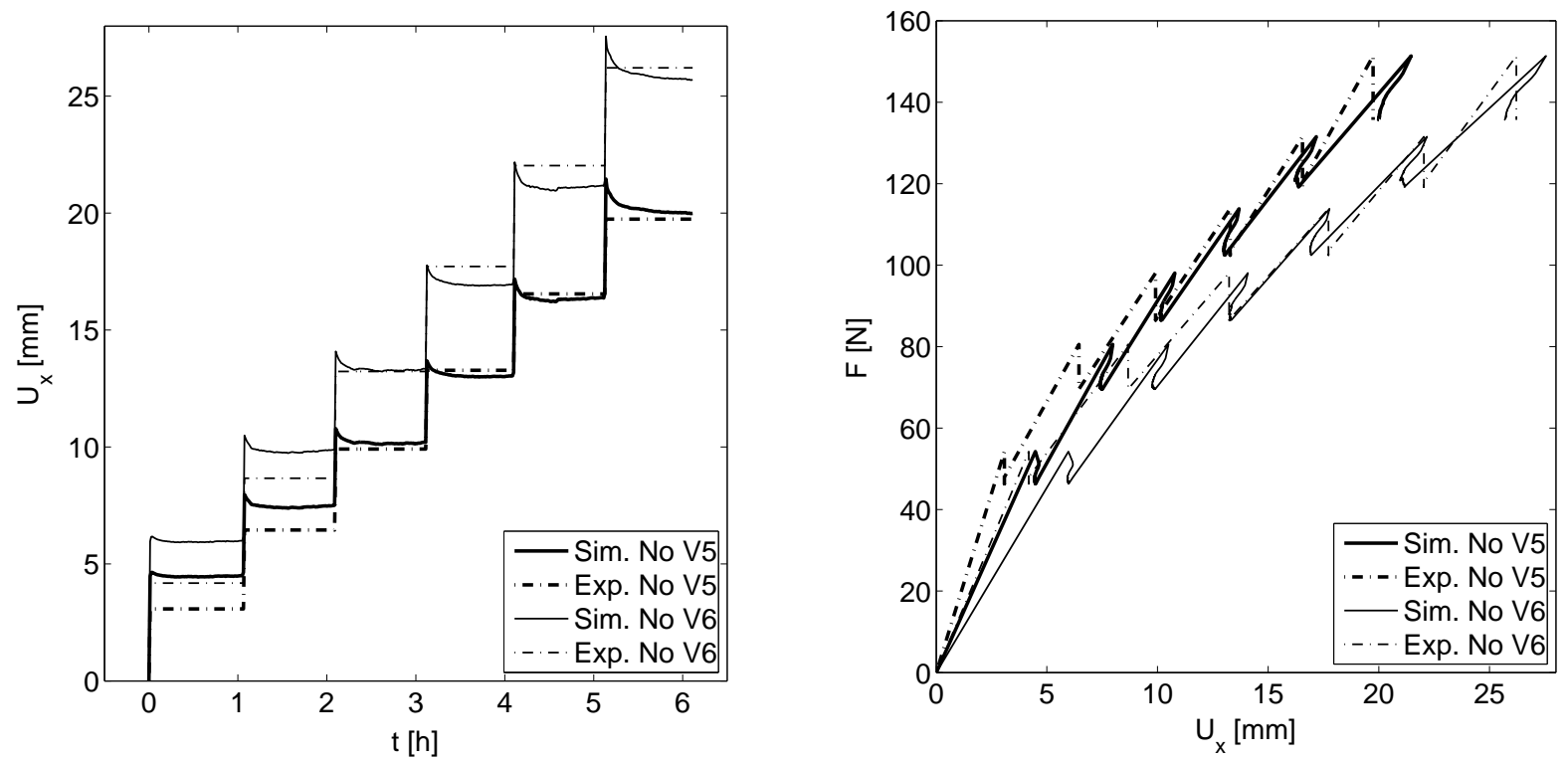

Figure 7.58: Extended verification for test $C$; displacements in longitudinal direction.
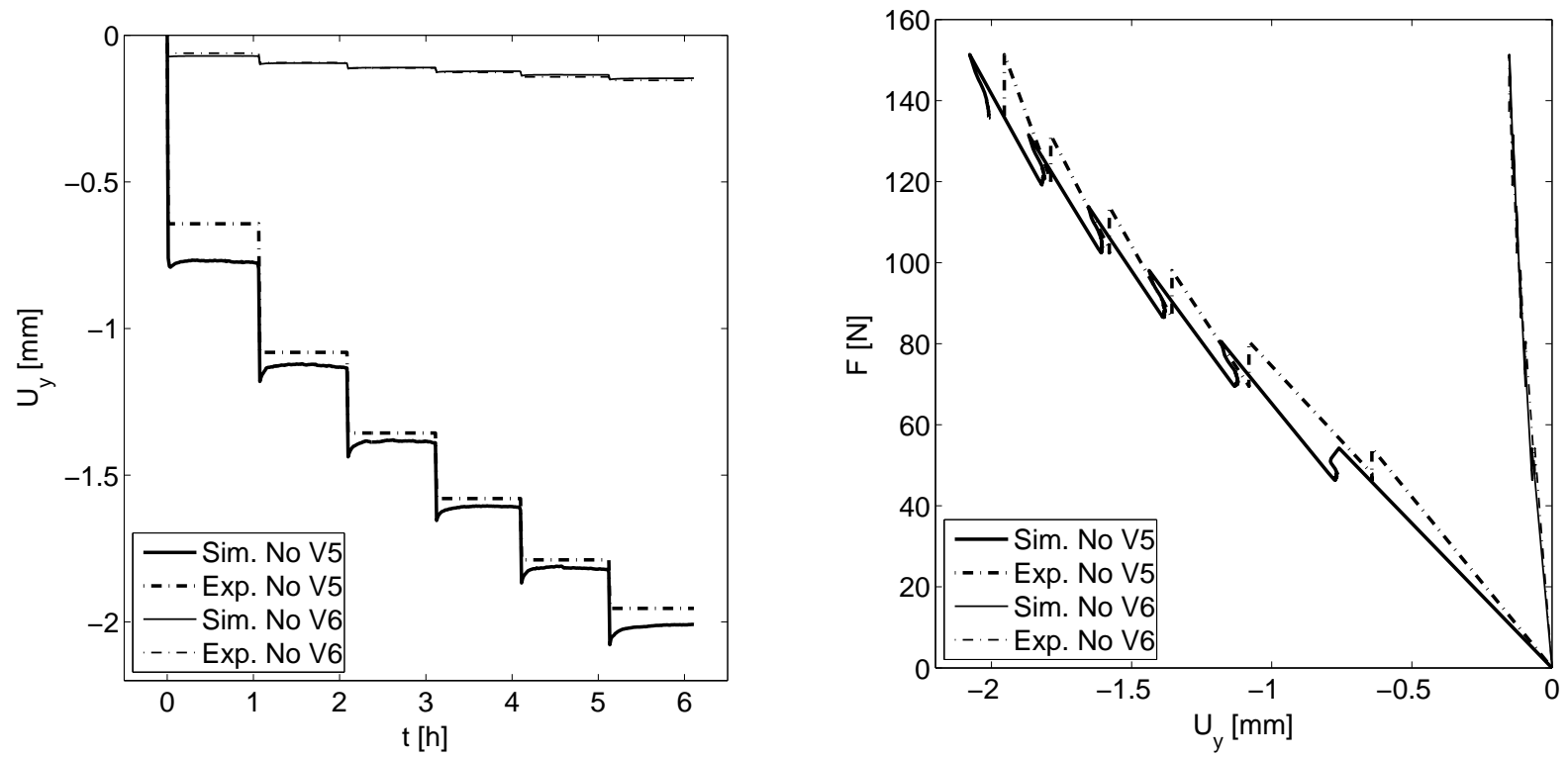

Figure 7.59: Extended verification for test $C$; displacements in transverse direction. 


\section{Parameter identification for plasticity on the basis of real measurement data of aluminum alloy AISi9Cu3}

The parameter identification for metallic materials has been an important research area in the last years, whereby different constitutive routines, measurement techniques and materials have been considered (see, e.g. Mahnken and Stein ( [48], [47], [49]), Andresen et al. [1], Mahnken et. al. [45], Mahnken ( [40], [41], [42]) and Scheday [66]). The aim of the following study is to estimate the parameters of the aluminum alloy AlSi9Cu3. This material is intended for use in cast automotive applications and other allied industries. In contrast to the application of steel, the respective components are lighter, however, they are also more expensive and less buckling resistant. For the simulation of the mechanical material behavior for the dimensioning of components in lightweight construction the determination of the material parameters for a suitable material law is necessary.

As mentioned in the previous sections, the method of simultaneous identification of one single set of material parameters for multiple experiments can have different objectives. The main objective for the application of this method for the parameter identification of polyurethanes as described in the chapters 7.2 and 7.3 is to find a single set of parameters which enables the simulations to fit to the respective experiments for all tests in an average way. Thereby, the goal is to avoid overparametrization for a complex material law. For the case of the aluminum alloy considered in this chapter the main objective for the simultaneous identification for multiple experiments is to find a single set of parameters which fits to different specimen in an average way. In order to determine reliable parameters for the material, the necessity for the application of this procedure is given by different facts: The first reason is the scattering of experimental data between the different experiments due to inevitable inhomogeneities of the specimen resulting from the casting process. The second reason is the scattering of experimental data within each experiment due to inevitable uncertainties within the optical measurements of the very small displacements in the deformation range of the considered aluminum alloy. In addition, uncertainties of the force control of the testing machine have to be taken into account.

\subsection{Constitutive equations for von Mises plasticity}

The mechanical behavior of the considered aluminum alloy is simulated with von Mises plasticity with isotropic hardening. Since the displacements in the tensile tests are very 
small, a small strain theory is considered. Thereby, the actual total strain field yields

$$
\varepsilon=\frac{1}{2}\left[\nabla_{\boldsymbol{x}}^{\mathrm{T}} \boldsymbol{u}+\nabla_{\boldsymbol{x}} \boldsymbol{u}\right]
$$

with the displacement field $\boldsymbol{u}$. Moreover, an additive split into an elastic part $\varepsilon^{\mathrm{e}}$ and a plastic part $\varepsilon^{\mathrm{p}}$ is assumed with $\varepsilon=\varepsilon^{\mathrm{e}}+\varepsilon^{\mathrm{p}}$. The constitutive equations are summarized in table 8.1. For further elaborations see, e.g. Simo and Hughes [70]. For information on the subject of constitutive models within the context of geometrically nonlinear anisotropic elasto-plasticity, the reader is referred to the contributions by, for instance, Menzel [52] and Menzel and Steinmann ( [55], [54], [53]).

Table 8.1: Small strain von Mises plasticity.

(1) Kinematics:

(2) Internal variable:

(3) Free energy:

4) Real stress:

(5) Yield function:

(6) Isotropic hardening:

(7) Evolution equations:

(8) Kuhn-Tucker conditions:

(9) Material parameters:

$$
\varepsilon=\varepsilon^{\mathrm{e}}+\varepsilon^{\mathrm{p}}
$$

$$
\alpha, \varepsilon^{\mathrm{p}}
$$$$
\Psi=\Psi\left(\varepsilon^{\mathrm{e}}, \alpha\right)=\Psi\left(\varepsilon^{\mathrm{e}}\right)+\Psi(\alpha)
$$$$
\Psi\left(\varepsilon^{\mathrm{e}}\right)=\frac{K}{2}\left[\varepsilon^{\mathrm{e}}: \boldsymbol{I}\right]^{2}+\mu\left[\varepsilon^{\mathrm{edev}}\right]^{2}: \boldsymbol{I}
$$$$
\boldsymbol{\sigma}=3 K \varepsilon^{\mathrm{sph}}+2 \mu\left[\varepsilon^{\mathrm{dev}}-\varepsilon^{\mathrm{p}}\right]
$$$$
\phi=\left\|\boldsymbol{\sigma}^{\operatorname{dev}}\right\|-\sqrt{2 / 3} h
$$$$
h=y_{0}+H \alpha+\left[y_{\infty}-y_{0}\right][1-\exp (-\omega \alpha)]
$$$$
\dot{\varepsilon}^{\mathrm{p}}=\dot{\lambda} \boldsymbol{n} \quad \dot{\alpha}=\dot{\lambda} \sqrt{2 / 3}
$$$$
\boldsymbol{n}=\frac{\boldsymbol{\sigma}^{\mathrm{dev}}}{\left\|\boldsymbol{\sigma}^{\mathrm{dev}}\right\|}
$$

$$
\dot{\lambda} \geq 0, \phi \leq 0, \dot{\lambda} \phi=0
$$

$$
\boldsymbol{\kappa}=\left[K, \mu, y_{0}, y_{\infty}, \omega, H\right]^{\mathrm{T}}
$$

The material parameters are the bulk modulus $K$, the shear modulus $\mu$, the initial yield limit $y_{0}$, the saturated yield limit $y_{\infty}$, the exponential hardening modulus $\omega$ and the linear hardening modulus $H$. Note that in the later identification procedure the parameter set $\boldsymbol{\kappa}=\left[E, \nu, y_{0}, y_{\infty}, \omega, H\right]^{\mathrm{T}}$ is considered. Thereby, the relationships $E=9 K \mu /[3 K+\mu]$ and $\nu=[3 K-2 \mu] /[6 K+2 \mu]$ hold. 


\subsection{Specimens}

The blank specimens are manufactured by die casting. Within this process the molten metal is forced under high pressure into the cavities of steel molds (dies). The produced metal parts are comparatively precise and have a very smooth surface.

The delivered raw specimens have been processed by the metal shop of the University of Kaiserslautern according to the engineering drawing in figure 8.1. In the zone of the hole

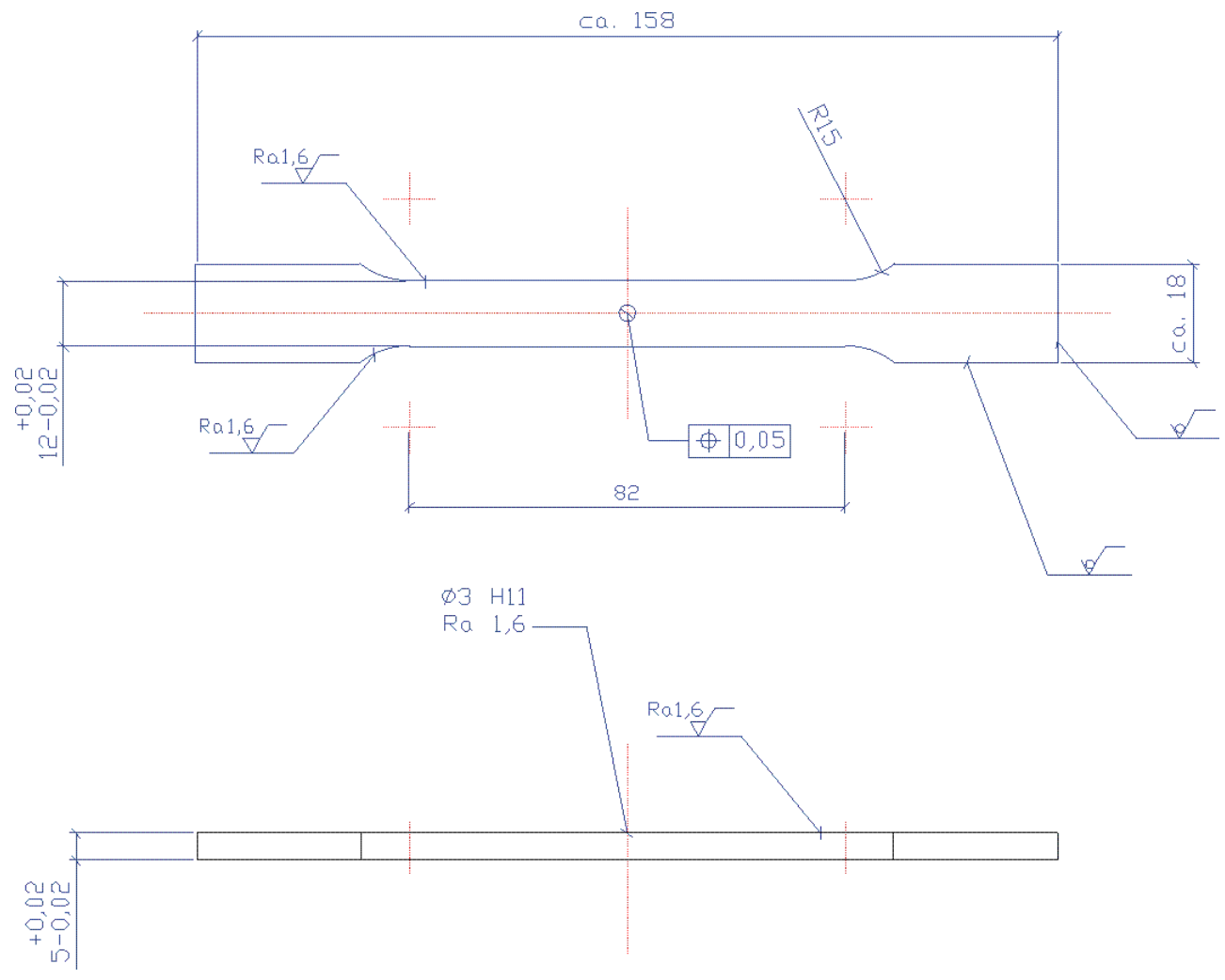

Figure 8.1: Geometry of the specimen.

an inhomogeneous state of stress is induced and therewith the location of the necking is defined. In a sufficient distance to the hole an approximately homogeneous state of stress can be assumed. For the measurement of the displacement fields by means of the two-dimensional image correlation photogrammetry a stochastic pattern is applied onto the considered area of the specimen's surface.

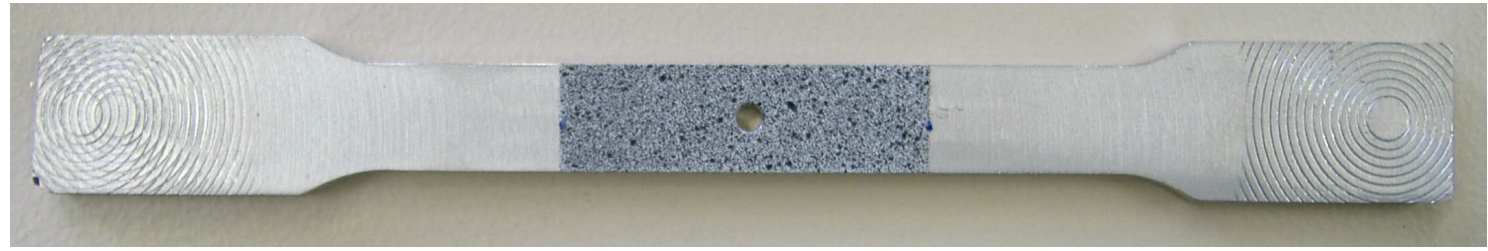

Figure 8.2: Specimen with stochastic pattern. 


\subsection{Experiments}

For the experiments the servo-hydraulic tensile testing machine SCHENK Hydropuls PSA 10 is used. This machine has a capacity of $100 k N$ in static and $80 k N$ in dynamic loading. The maximal stroke is $100 \mathrm{~mm}$. The loading on the specimen is applied by hydraulic clamping. The experiments are conducted at room temperature. Thermal effects like the generation of heat during the deformation process are not considered. In order to avoid temperature effects due to the lighting of the specimen at the measurements, two cold lights (Dedocool, 250W Quartz Halogen ELC Type) are set up. The CCD camera (Vosskühler 1300, resolution 1024 x 1280 pixels) with $50 \mathrm{~mm}$ objective (Schneider Kreuznach) is positioned on a stand vertical to the direction of the movement. The twodimensional displacement field is computed by using the photogrammetric evaluation procedure ARAMIS (Version 5.4).

For the parameter identification of the cast aluminum alloy the measurement of the displacement fields of three specimen $P 83, P 84$, and $P 85$ are determined in three tests $A(P 83), B(P 84)$, and $C(P 85)$. Thereby, in order to activate all material parameters in the later identification routine and to differentiate between the purely elastic and elastoplastic parts of the deformation process, a test procedure with an (elastic) unloading is chosen: The three tests are conducted in force control with a rate of $45 \mathrm{~N} / \mathrm{s}$ to a maximal force load of $9000 N$ (maximum tensile load per square unit of original cross section: $200 \mathrm{MPa}$ ). Then the specimen is unloaded with a rate of $-45 \mathrm{~N} / \mathrm{s}$ to $0 \mathrm{~N}$. Every $5 \mathrm{~s}$ an image of the specimen is taken by the CCD cameras for the displacement measurement (a total number of 80 images per specimen). The total time for each test is $320 \mathrm{~s}$.

The experimental results are the force-time curves (see figure 8.3) - whereby $\mathrm{F}$ is the total force in loading direction - and the two-dimensional displacements of the measurement
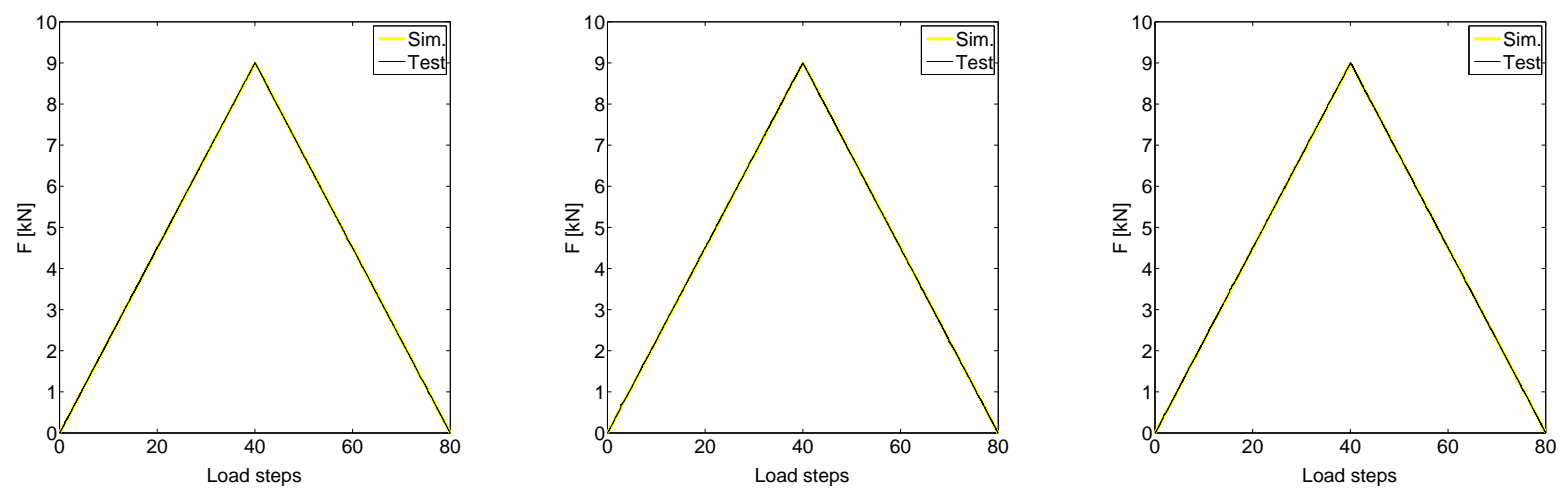

Figure 8.3: Force vs. load steps curves for the tests of the specimens $P 83$ (left), $P 84$ (center) and P85 (right). These data are used for the (force-) control of the corresponding simulations.

points along the longitudinal $(x)$ and transverse $(y)$ direction on the considered part of the surface of the specimen. It needs to be mentioned that the displacement data of the measurement points directly at the hole is relatively inaccurate. These measurement points are discarded for the following parameter identification. Moreover, significant noise within the measurement of each individual experiment occurs. As already mentioned this is due to the very small displacements and the resulting inevitable inaccuracies in the 
optical measurements.

The experimental data was determined by M. Bosseler of the 'Institute of Resource centered Product Development' under the supervision of Prof. Dr.-Ing. R. Renz, University of Kaiserslautern. The experiments were conducted by the Chair for Applied Mechanics, University of Kaiserslautern.

\subsection{Interpolation of experimental data}

For the parameter identification effects like the slipping in the clamping and the influence of the stiffness of the testing machine have to be excluded. Therefore, the measured and the simulated displacements relative to an identification node are used within the identification algorithm. Hereby, only the displacement field of a section of the specimen needs to be measured. Moreover, the discretization of the model of the specimen only requires a length of the FE model until an approximately homogeneous state of stress in a sufficient distance to the hole can be assumed. This allows an equivalent force transmission in the FE simulation in analogy to the experiment. For the simulation of the tests a FEM discretization of the specimen with 1500 eight-node enhanced elements (Q1E9) (see Simo and Armero [69]) is used. Thereby, due to the symmetry of the specimen only half of the width, half of the length and half of the depth is discretized (see figure 8.6). In addition to the symmetry conditions the boundary conditions for both ending planes normal to the longitudinal direction are chosen in correspondence with the experiments. The nodes on the plane $\left.x\right|_{t_{0}}=-13(\mathrm{~mm})$ are fixed in longitudinal direction according to the approximately homogeneous stress field at the respective position of the specimen in the experiment. For the nodes on the plane $\left.x\right|_{t_{0}}=0$ in longitudinal direction uniaxial normal forces are applied, whereby warping of the plane is prevented. Rigid body motion is excluded.

Since the coordinates of the measurement points generally do not coincide with the coordinates of the identification nodes, the measured displacements are linearly interpolated on the identification nodes by triangulation (see section 4.3). For the triangulation the displacements of the three measurement points being nearest to the respective identification node are used. In order to make the three tests $A(P 83), B(P 84)$, and $C(P 85)$ comparable for the later identification, throughout identical identification nodes are chosen for all three tests. Figure 8.4 shows the interpolation of the measured displacements on the selected identification nodes for the specimen $P 83$. After the execution of the interpolations for the tests $A(P 83), B(P 84)$, and $C(P 85)$ for all specimen and for all 80 measured load steps the following 159 identification nodes are chosen (see figure 8.5).

\subsection{Parameter identification by means of relative displacements}

As described above, for the parameter identification the measured and simulated displacements relative to an identification node $I_{r e l}$ are used. In doing so, on the one hand inaccuracies due to the slipping in the clamping and the influence of the stiffness of the 


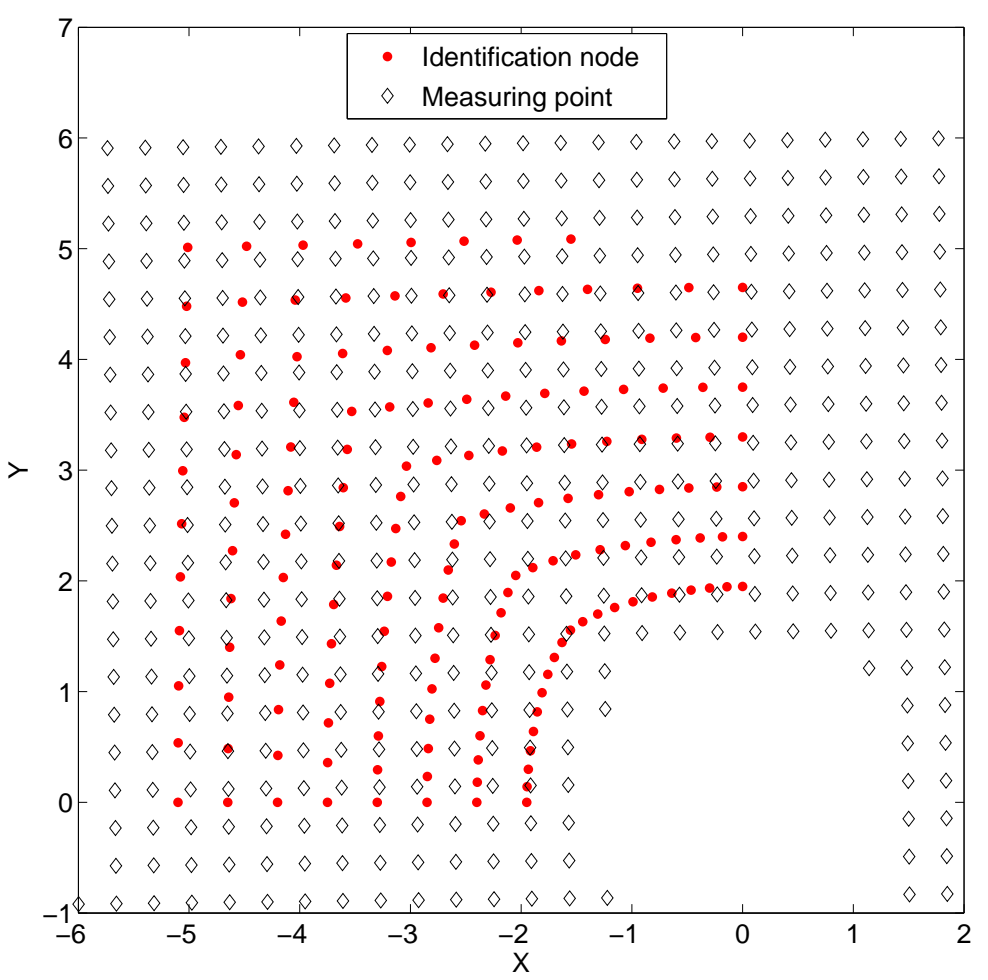

Figure 8.4: Interpolation of the measured displacements on the selected identification nodes for the specimen $P 83$.

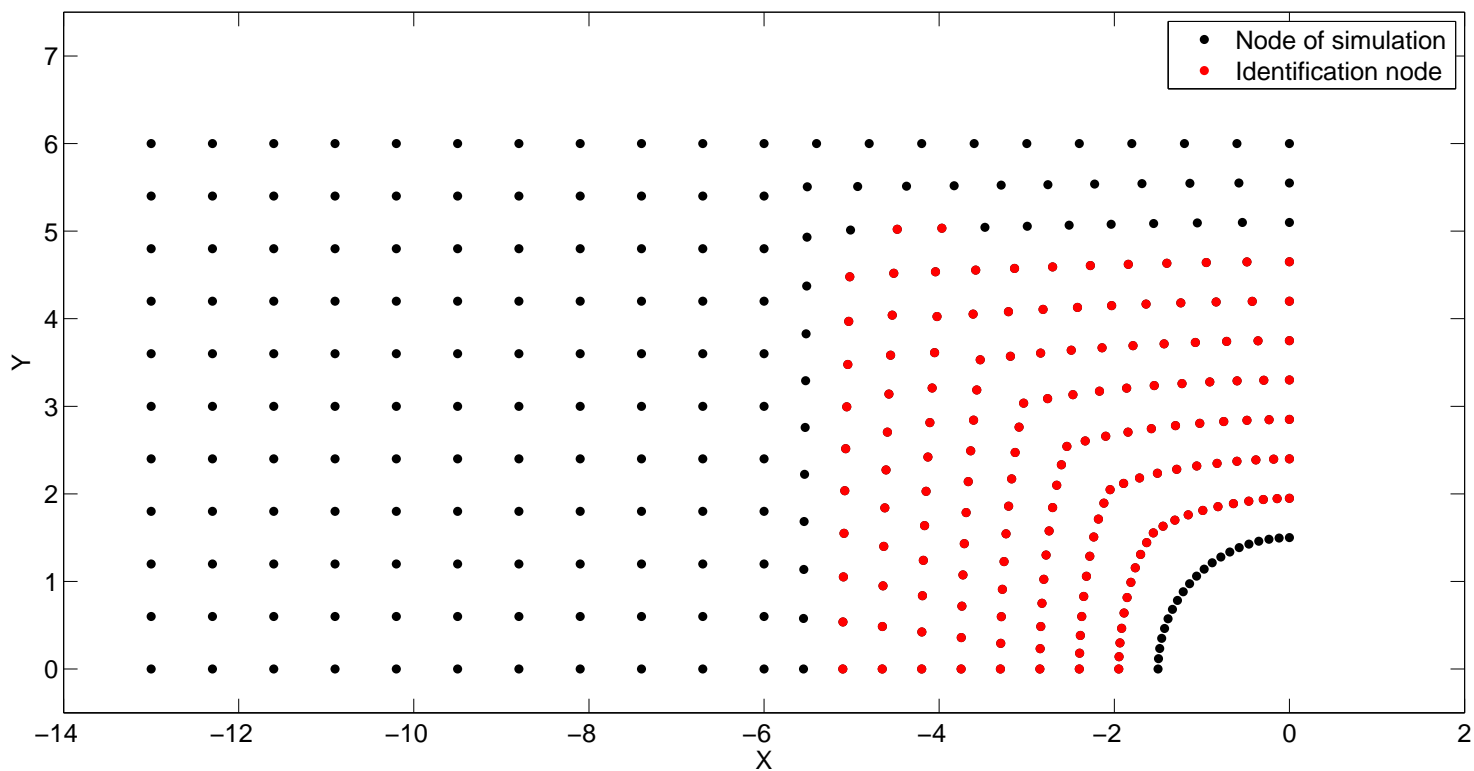

Figure 8.5: Choice of the $N=159$ identification nodes for the tests of the specimens $P 83, P 84$, and P85. 
testing machine can be excluded. On the other hand, therewith, only a section of the specimen has to be discretized which means a significant saving of computing time within the FE calculations. Moreover, the complicated measuring of the exact position of the specimen in the clamping and the complicated simulation of the respective force transmission for a simulation of the entire specimen in the FE simulation is not necessary.

For an uniaxial tensile test with a measurement of two-dimensional displacements in length and width direction, the chosen identification node $I_{r e l}$ must be positioned on the longitudinal axis (in the middle of the width) of the symmetric specimen. At every load step $j$ and for all identification nodes $i$ the differences of the displacements to every identification node $I_{\text {rel }}$ are formed (for $I_{r e l}$ let $i=i_{r}$ ). Therefore, at load step $j$ the relative displacements for the FE calculation are $\overline{\boldsymbol{u}}_{i_{r} j}(\boldsymbol{\kappa})-\overline{\boldsymbol{u}}_{i j}(\boldsymbol{\kappa})$, and for the interpolated measured displacements they are $\overline{\boldsymbol{u}}_{i_{r j}}^{\text {exp }}-\overline{\boldsymbol{u}}_{i j}^{\text {exp }}$. Hence, for the parameter identification of a single test the following least squares approach is applied

$$
f(\boldsymbol{\kappa})=\frac{1}{2} \sum_{i=1}^{T} \sum_{j=1}^{N}\left[\left[\overline{\boldsymbol{u}}_{i_{r} j}(\boldsymbol{\kappa})-\overline{\boldsymbol{u}}_{i j}(\boldsymbol{\kappa})\right]-\left[\overline{\boldsymbol{u}}_{i_{r} j}^{\exp }-\overline{\boldsymbol{u}}_{i j}^{\text {exp }}\right]\right]^{2},
$$

for which the gradient yields

$$
\partial_{\boldsymbol{\kappa}} f(\boldsymbol{\kappa})=\sum_{i=1}^{T} \sum_{j=1}^{N}\left[\left[\overline{\boldsymbol{u}}_{i_{r} j}(\boldsymbol{\kappa})-\overline{\boldsymbol{u}}_{i j}(\boldsymbol{\kappa})\right]-\left[\overline{\boldsymbol{u}}_{i_{r j}}^{e x p}-\overline{\boldsymbol{u}}_{i j}^{e x p}\right]\right] \cdot\left[\partial_{\boldsymbol{\kappa}} \overline{\boldsymbol{u}}_{i_{r} j}(\boldsymbol{\kappa})-\partial_{\boldsymbol{\kappa}} \overline{\boldsymbol{u}}_{i j}(\boldsymbol{\kappa})\right]
$$

Remark 8.5.1 Since the order of magnitude of the parameters differs, a parameter weight $\boldsymbol{V}^{(j)}$ is employed to the calculation of the iteration step (see also equation 4.10)

$$
\Delta \boldsymbol{\kappa}=\boldsymbol{\kappa}^{(j+1)}-\boldsymbol{\kappa}^{(j)}=-\boldsymbol{V}^{(j)} \cdot\left[\alpha^{(j)} \boldsymbol{H}_{L M}^{(j)} \cdot \partial \boldsymbol{\kappa} f\left(\boldsymbol{\kappa}^{(j)}\right)\right]
$$

Conceptually speaking, the diagonal matrix $\boldsymbol{V}^{(j)}$ weights the step $\Delta \kappa_{1}$ for each parameter corresponding to the magnitude of $\kappa_{1}^{(j)}$, whereby

$$
\boldsymbol{V}^{(j)}=\operatorname{diag}\left(a_{1}^{(j)}, \ldots, a_{11}^{(j)}\right)
$$

For the elaborated iteration processes - presented in the following section - we choose $a_{1}=1000, a_{2}=0.01, a_{3}=10, a_{4}=10, a_{5}=1$, and $a_{6}=100$.

Remark 8.5.2 The parameter sensitivities for the identification iteration by means of the Levenberg-Marquardt method $\partial_{\boldsymbol{\kappa}} \overline{\boldsymbol{u}}_{i j}(\boldsymbol{\kappa})$ are calculated numerically by

$$
\partial_{\kappa_{m}} \overline{\boldsymbol{u}}_{i j}(\boldsymbol{\kappa}) \approx \frac{\overline{\boldsymbol{u}}_{i j}\left(\boldsymbol{\kappa}+\epsilon \boldsymbol{V} \cdot \boldsymbol{e}_{m}\right)-\overline{\boldsymbol{u}}_{i j}(\boldsymbol{\kappa})}{\epsilon}
$$

with $m=1,2, \ldots, 6$ for the six parameters of the applied elasto-plastic material law. Here $\boldsymbol{e}_{m}$ is an unit vector with the entry 1 at position $m$ and the entries 0 for the five other positions. Moreover $\boldsymbol{V}$ is the parameter weight as described in equation 8.4. The numerical pertubation is computed with $\epsilon=10^{-7}$. 


\subsection{Parameter identification for experimental results of AlSi9Cu3}

The objective function (8.2) is, in general, non-convex which means that in addition to the global minimum local minima might occur. Therefore, - for the parameter identifications described in the following subsections - the hybrid method of combining the LevenbergMarquardt method with the stochastic method of testing different start parameters is applied. Basically it can be observed, that for each new starting point a number of eight iteration steps are enough to detect if the starting point leads to a local minimum or whether this iteration can be discarded due to divergence. If the iteration process is trapped in a local minimum, the iteration is repeated with new starting points by changing individual parameters manually which is on the one hand a check for the robustness of the result. On the other hand this can also lead to another local minimum with a smaller value for the weighted sum of squared differences.

In the following the results of the parameter identifications for the tests $A(P 83), B(P 84)$, and $C(P 85)$ are presented. In subsection 8.6.1 the identification for the combination of the three tests is shown. In the subsections 8.6.2, 8.6.3, and 8.6.4 the respective individual identifications for the single tests $A(P 83), B(P 84)$, and $C(P 85)$ follow. By comparison of the different sets of parameters for the respective identifications it is investigated, how far the identification routine for the simultaneous identification for all three tests has to average the parameters (see subsection 8.6.5).

Figure 8.6 shows the FE model being applied for all respective parameter identifications. Thereby, $I_{r e l}$ is the identification node for the calculation of relative displacements. For the respective verifications the identification nodes $1-5$ are used for the representation of the relative displacement vs. load step curves and the force vs. relative displacement curves of the FE calculation with optimal material parameters in comparison to the respective interpolated measurements.

\subsubsection{Identification for the combination of the tests of the specimens P83, P84, and P85}

For this parameter identification a weighted least squares sum in analogy to equation (8.2) with displacements relative to the identification node $I_{r e l}$ is used. Within the iteration 


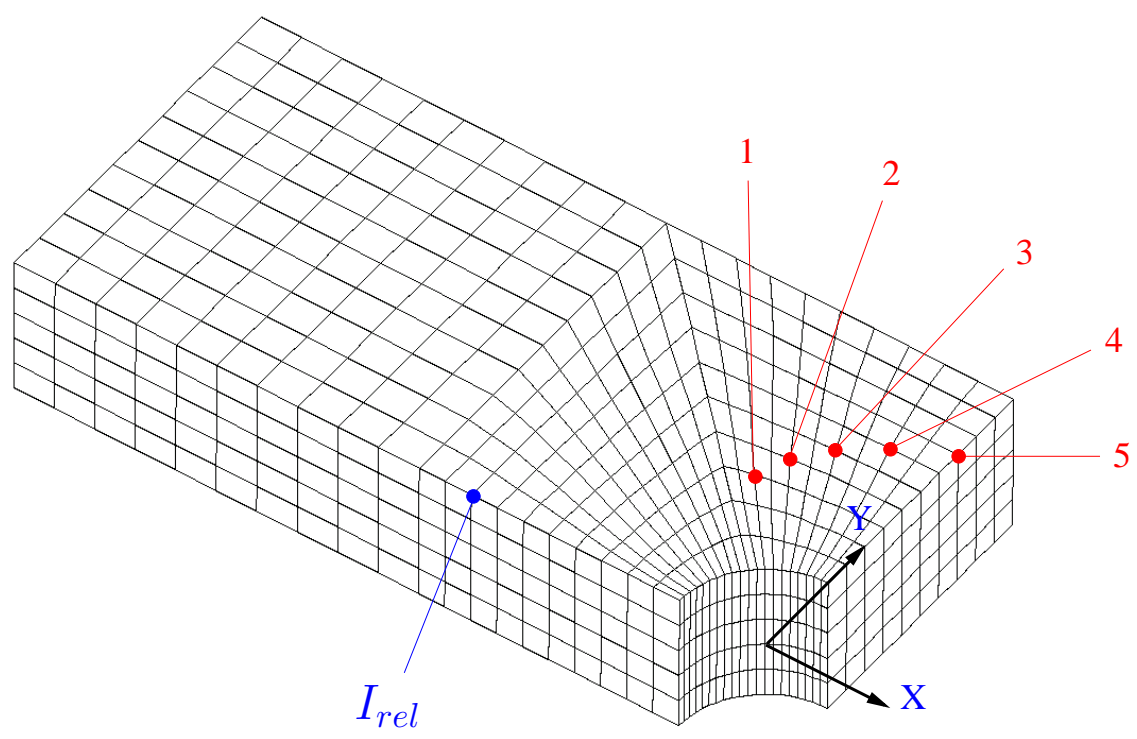

Figure 8.6: FEM model.

algorithm all three tests $A(P 83), B(P 84)$, and $C(P 85)$ are considered simultaneously.

$$
\begin{aligned}
f^{A B C}(\boldsymbol{\kappa})=\frac{1}{2} \sum_{i=1}^{N_{g}} & (\underbrace{\sum_{j=1}^{T_{A}}\left[\boldsymbol{W}_{i j} \cdot\left[\left[\overline{\boldsymbol{u}}_{i_{r} j}(\boldsymbol{\kappa})-\overline{\boldsymbol{u}}_{i j}(\boldsymbol{\kappa})\right]-\left[\overline{\boldsymbol{u}}_{i_{r} j}^{\text {exp }}-\overline{\boldsymbol{u}}_{i j}^{\text {exp }}\right]\right]^{2}\right.}_{A(P 83)} \\
& +\underbrace{\sum_{k=1}^{T_{B}}\left[\boldsymbol{W}_{i k} \cdot\left[\left[\overline{\boldsymbol{u}}_{i_{r} k}(\boldsymbol{\kappa})-\overline{\boldsymbol{u}}_{i k}(\boldsymbol{\kappa})\right]-\left[\overline{\boldsymbol{u}}_{i_{r} k}^{\text {exp }}-\overline{\boldsymbol{u}}_{i k}^{\text {exp }}\right]\right]^{2}\right.}_{B(P 84)} \\
& +\underbrace{\sum_{l=1}^{T_{C}}\left[\boldsymbol{W}_{i l} \cdot\left[\left[\overline{\boldsymbol{u}}_{i_{r} l}(\boldsymbol{\kappa})-\overline{\boldsymbol{u}}_{i l}(\boldsymbol{\kappa})\right]-\left[\overline{\boldsymbol{u}}_{i_{r} l}^{\text {exp }}-\overline{\boldsymbol{u}}_{i l}^{\text {exp }}\right]\right]\right]^{2}}_{C(P 85)})
\end{aligned}
$$

Thereby, $\boldsymbol{W}_{i j}, \boldsymbol{W}_{i k}$, and $\boldsymbol{W}_{i l}$ are weighting matrices with $\boldsymbol{W}_{i j}=\operatorname{diag}\left(\left[W_{i j}\right]_{1},\left[W_{i j}\right]_{2}\right)$, $\boldsymbol{W}_{i k}=\operatorname{diag}\left(\left[W_{i k}\right]_{1},\left[W_{i k}\right]_{2}\right)$, and $\boldsymbol{W}_{i l}=\operatorname{diag}\left(\left[W_{i l}\right]_{1},\left[W_{i l}\right]_{2}\right)$. Therefore, $\left[W_{i j}\right]_{1},\left[W_{i k}\right]_{1}$, and $\left[W_{i l}\right]_{1}$ are the weights for the respective relative displacements in longitudinal direction. As well $\left[W_{i j}\right]_{2},\left[W_{i k}\right]_{2}$, and $\left[W_{i l}\right]_{2}$ are the weights for the respective relative displacements in transverse direction. For the simulation of the displacement fields $\overline{\boldsymbol{u}}_{i j}(\boldsymbol{\kappa}), \overline{\boldsymbol{u}}_{i k}(\boldsymbol{\kappa})$ and $\overline{\boldsymbol{u}}_{i l}(\boldsymbol{\kappa})$ the FEM simulation is force-controlled based on the measured force signals shown in figure 8.3. Due to the equal number of load steps $T_{A}=T_{B}=T_{C}=80$ for the three tests - as well as equal numbers of identification nodes $N_{g}=159$ - all tests are weighted equally for all load steps. The similar dimensions of the displacements in length and width 
direction make the weighting unnecessary in this regard. Hence, we assume

$$
\boldsymbol{W}_{i j}=\boldsymbol{W}_{i k}=\boldsymbol{W}_{i l}=\operatorname{diag}(1,1) .
$$

The number of terms in the least square sum adds up to $3 * 159 * 80 * 2=76320$ per iteration step of the optimization algorithm.

The smallest of all investigated local minima in $\boldsymbol{\kappa}=\boldsymbol{\kappa}_{l m_{A B C}}$ is shown in table 8.2.

Table 8.2: Parameter set $\boldsymbol{\kappa}=\boldsymbol{\kappa}_{l m_{A B C}}$.

\begin{tabular}{|l|l|l|l|l|l|}
\hline$E[\mathrm{MPa}]$ & $\nu$ & $y_{0}[\mathrm{MPa}]$ & $y_{\infty}[\mathrm{MPa}]$ & $\omega$ & $H[\mathrm{MPa}]$ \\
\hline \hline 74313.9780 & 0.3258 & 132.7606 & 167.0247 & 225.4131 & 7208.3051 \\
\hline
\end{tabular}

In the identification iteration some instabilities occurred which is - as already described in section 4.5 - a common problem in parameter estimation. The consequence of these instabilities is that there exist several parameter sets which lead to a insignificantly higher least squares sum. In order to evaluate the significance for the quality of the solution, the correlation matrix for the optimal parameters shown in table 8.3 (see also equation 4.14) is considered.

Table 8.3: Correlation matrix for the set of optimal parameters for the combination of the tests $A(P 83)$, $B(P 84)$, and $C(P 85)$.

\begin{tabular}{|r||l|l|l|l|l|l|}
\hline & $E$ & $\nu$ & $y_{0}$ & $y_{\infty}$ & $\omega$ & $H$ \\
\hline \hline$E$ & 1.000 & 0.239 & -0.431 & 0.105 & -0.015 & -0.146 \\
\hline$\nu$ & 0.239 & 1.000 & -0.143 & 0.055 & 0.028 & -0.099 \\
\hline$y_{0}$ & -0.431 & -0.143 & 1.000 & -0.100 & -0.283 & 0.307 \\
\hline$y_{\infty}$ & 0.105 & 0.055 & -0.100 & 1.000 & -0.907 & -0.968 \\
\hline$\omega$ & -0.015 & 0.028 & -0.283 & -0.907 & 1.000 & 0.772 \\
\hline$H$ & -0.146 & -0.099 & 0.307 & -0.968 & 0.772 & 1.000 \\
\hline
\end{tabular}

In the identification procedure the parameters $E, \nu$, and $y_{0}$ are comparatively stable which is also confirmed by the small correlation numbers for the pairs of parameters which contain $E, \nu$, or $y_{0}$. The relatively unstable parameters are the parameters describing the hardening of the material, i.e. $y_{\infty}, \omega$, and $H$. This is due to the fact that the material does not have a (precisely) defined saturated yield limit and exhibits linear behavior of saturation hardening in the last part of the plastic deformation process which can be seen in the verifications presented in the following.

The verifications show that the local minimization of the weighted least squares functional delivers a parameter set which enables to fit the simulations to the respective experiments for all three tests in an average (weighted) way. Generally for the tests $A, B$ and $C$ a good qualitative and quantitative agreement between the experiment and the simulation can be observed. For the conclusions and information about the sum of squared differences for the above parameter identification see subsection 8.6.5. 


\section{Verification for test $\mathbf{A}$ (P83)}

Within this verification procedure the simulated data - calculated as based on the optimal parameters $\boldsymbol{\kappa}=\boldsymbol{\kappa}_{l m_{A B C}}$ for the combination of the tests $A(P 83), B(P 84)$, and $C(P 85)$ - is compared with the experimental data for test $A$. Figures 8.7-8.8 show the curves with (relative) displacement vs. load steps and force vs. (relative) displacement for the experiment and the simulation.
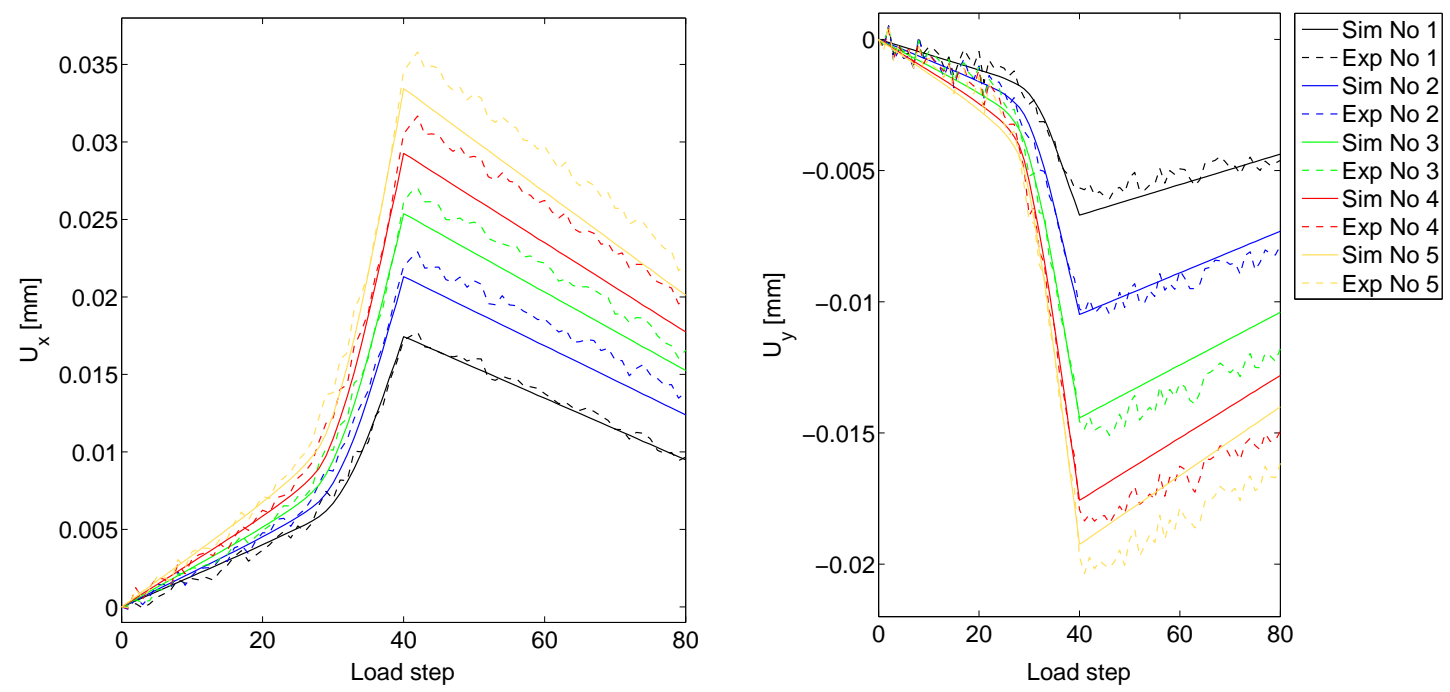

Figure 8.7: Verification for test $A$ with identified parameters for the combination of the tests $A, B, C$.
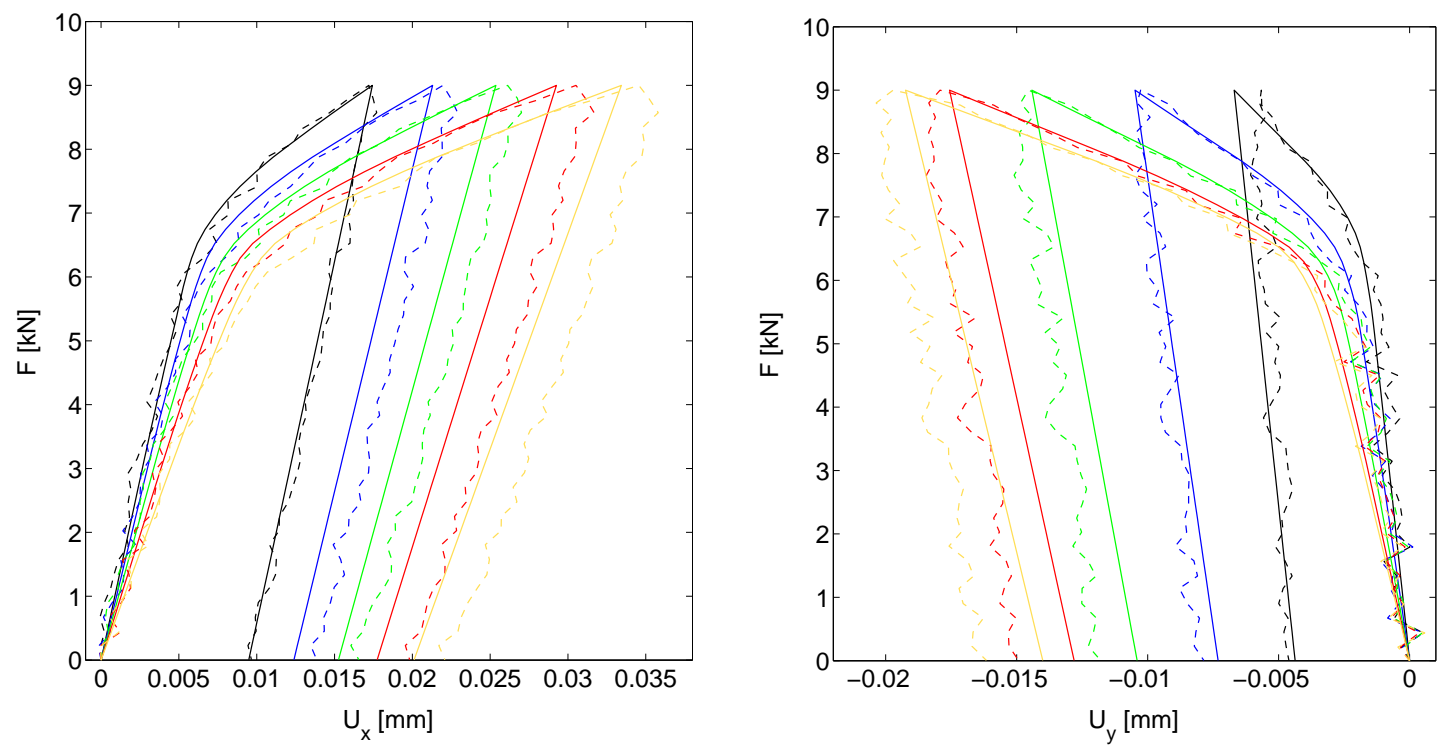

Figure 8.8: Verification for test $A$ with identified parameters for the combination of the tests $A, B, C$.

In figure 8.9 for all identification nodes the simulated positions for the load steps 20,40, 60, and 80 are plotted over the interpolated measured displacements for the FE model. Since the displacements are very small, for the representation in figure 8.9 the respective displacements are multiplied by factor 20 . 


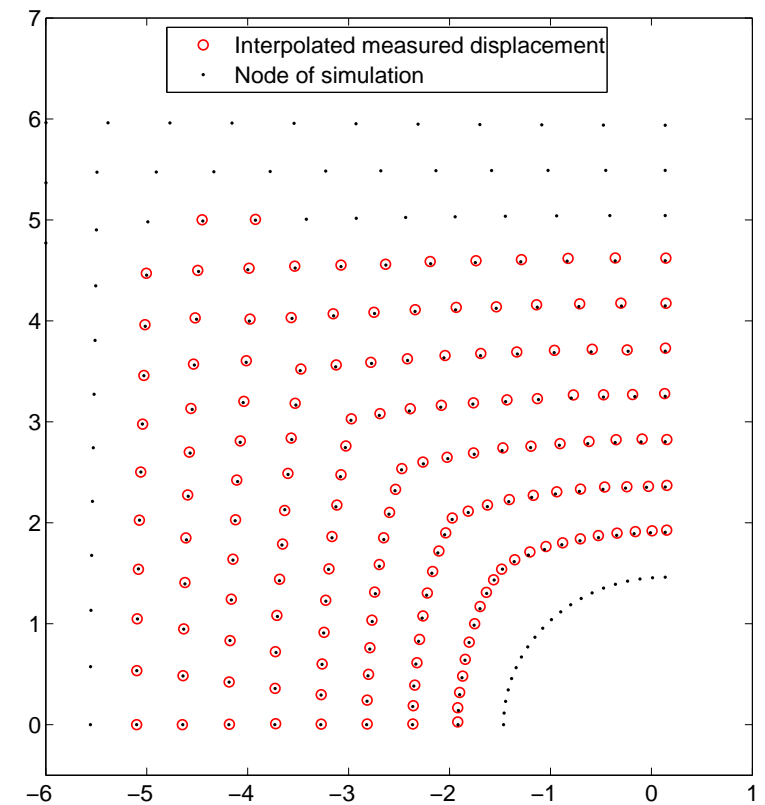

(a) Load step 20 .

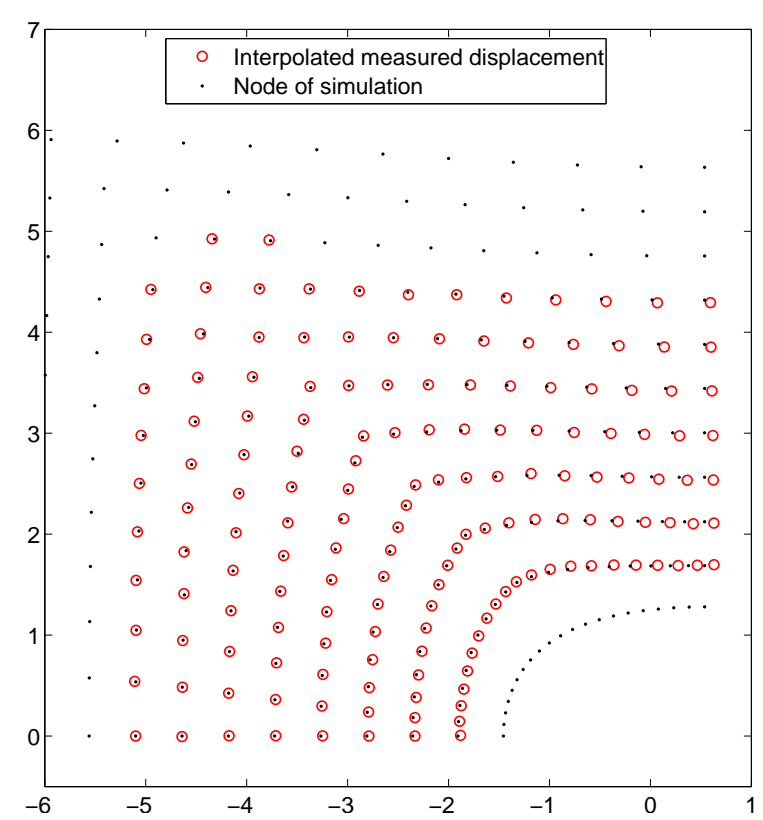

(c) Load step 60 .

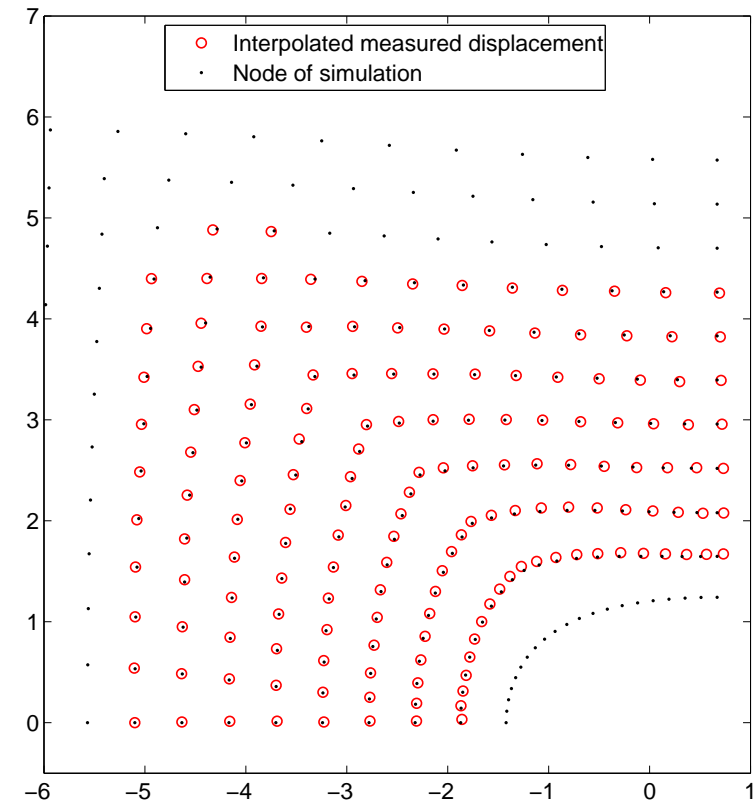

(b) Load step 40 .

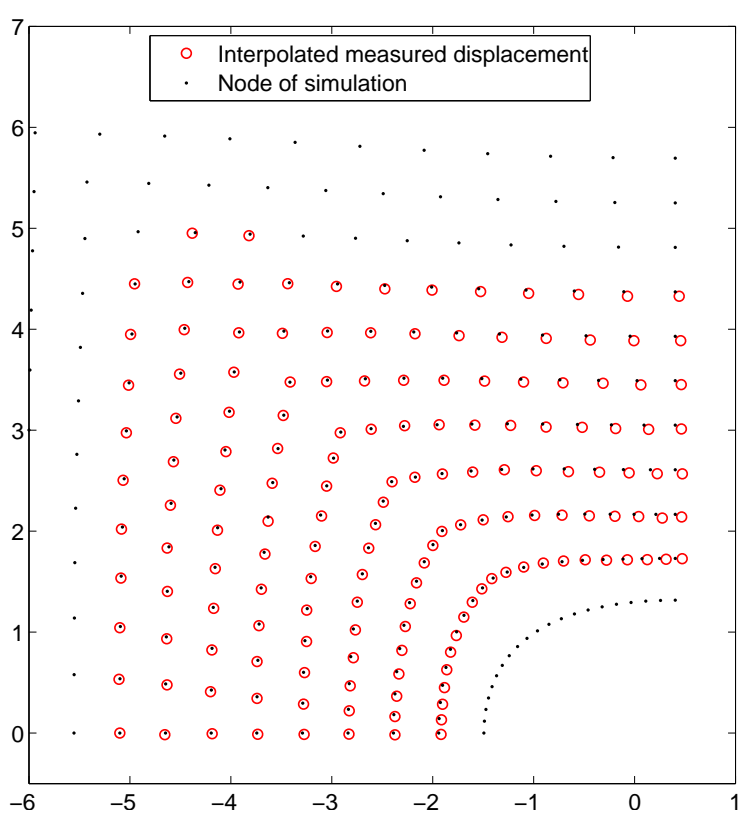

(d) Load step 80 .

Figure 8.9: Verification for test $A(P 83)$ with identified parameters for the combination of the tests $A(P 83), B(P 84)$, and $C(P 85)$; the displacements are multiplied by factor 20 for this representation. 


\section{Verification for test B (P84)}

Within this verification procedure the simulated data - calculated as based on the optimal parameters $\boldsymbol{\kappa}=\boldsymbol{\kappa}_{l m_{A B C}}$ for the combination of the tests $A(P 83), B(P 84)$, and $C(P 85)$ - is compared with the experimental data for test $B(P 84)$. Figures 8.10-8.11 show the curves with (relative) displacement vs. load steps and force vs. (relative) displacement for the experiment and the simulation.
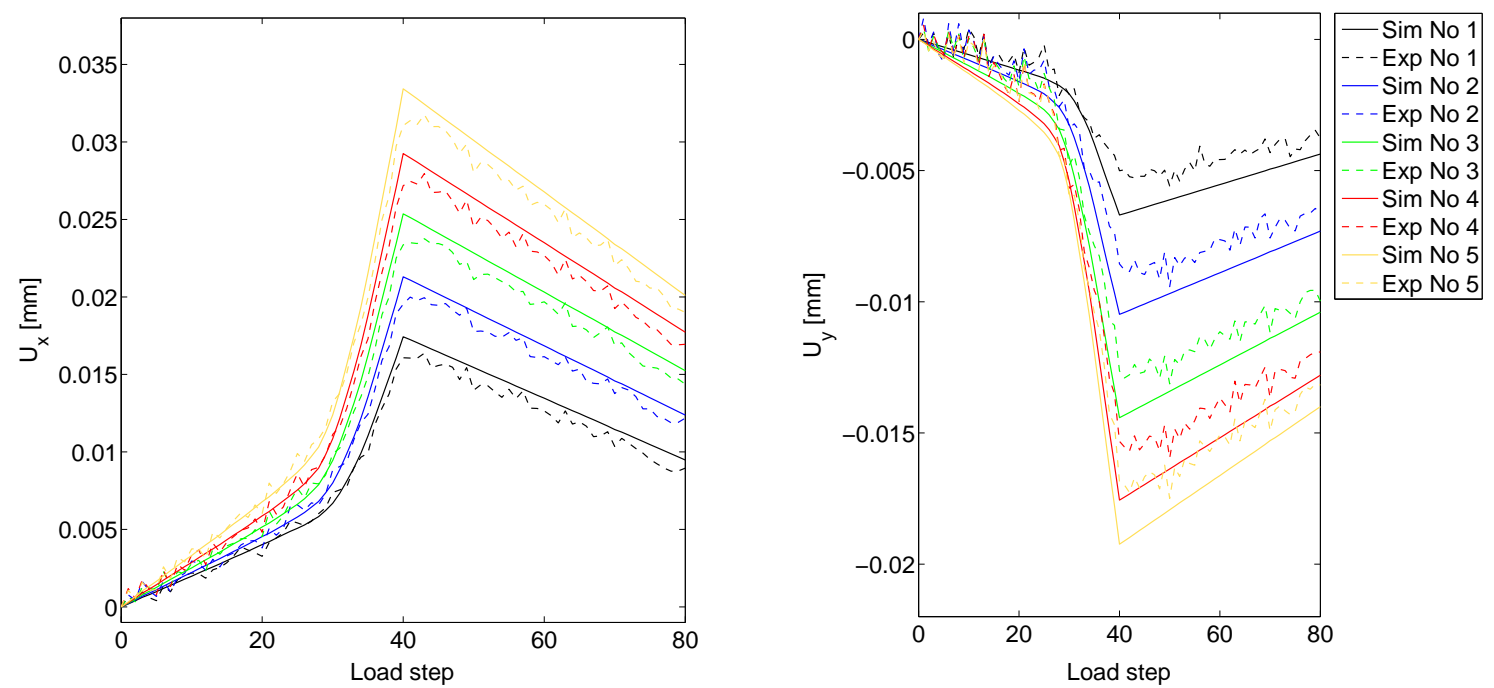

Figure 8.10: Verification for test $B$ with identified parameters for the combination of the tests $A, B, C$.
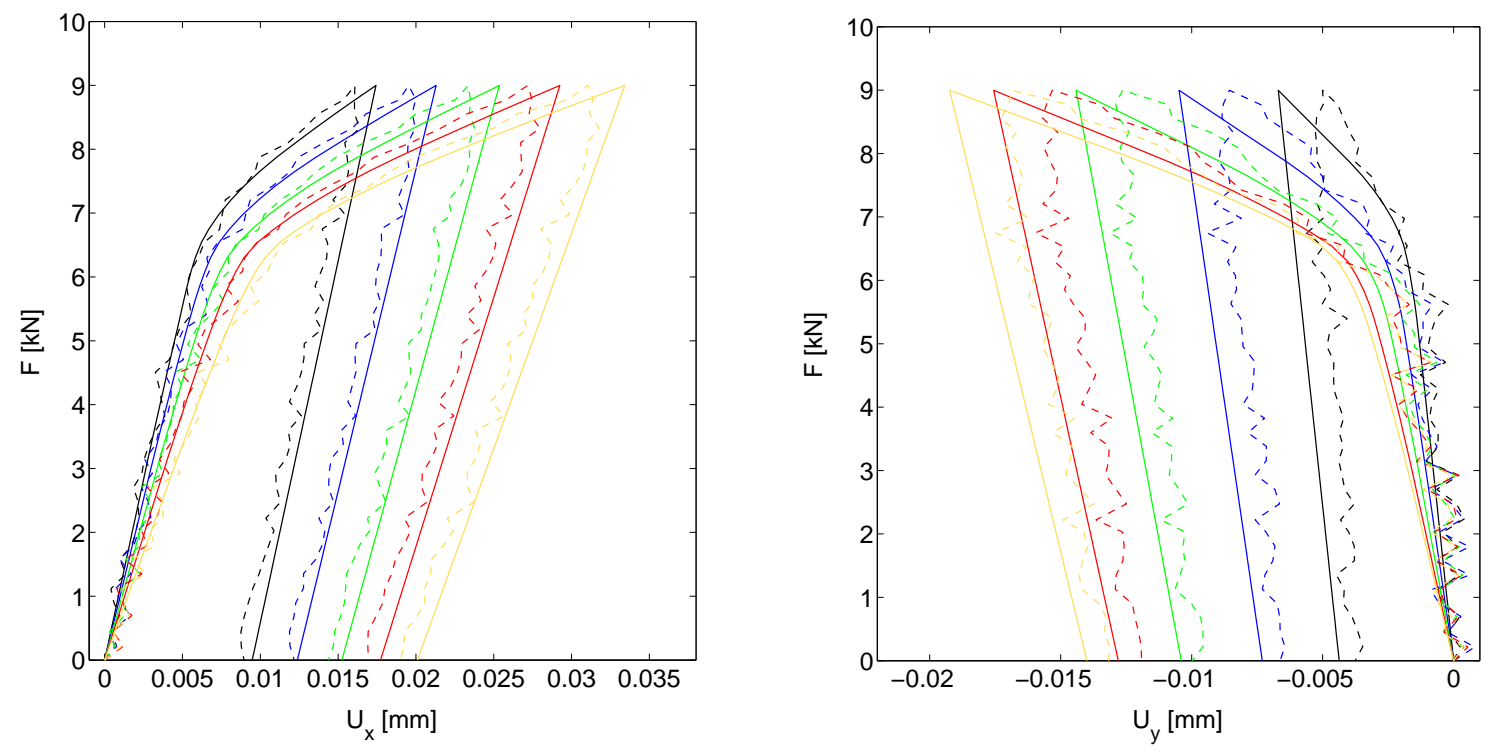

Figure 8.11: Verification for test $B$ with identified parameters for the combination of the tests $A, B, C$.

In figure 8.12 for all identification nodes the simulated positions for the load steps 20,40, 60, and 80 are plotted over the interpolated measured displacements for the FE model. For the representation in figure 8.12 the respective displacements are multiplied by factor 20. 


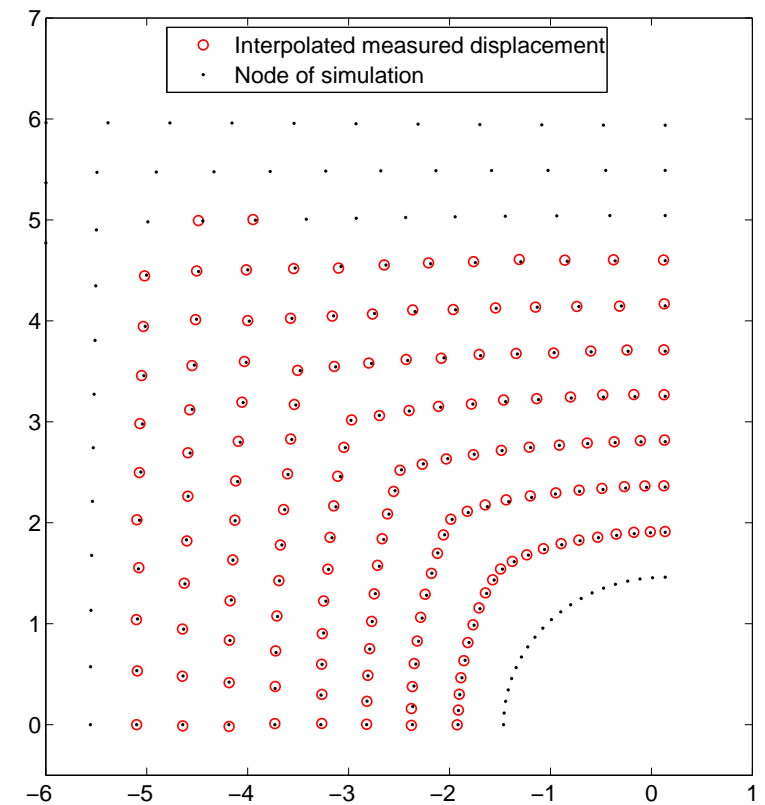

(a) Load step 20 .

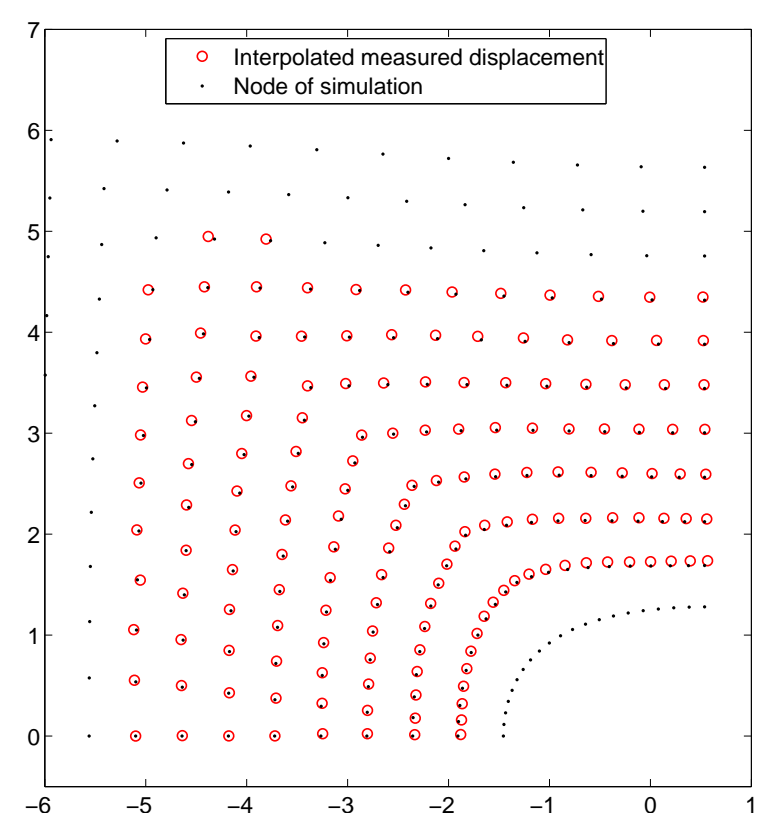

(c) Load step 60 .

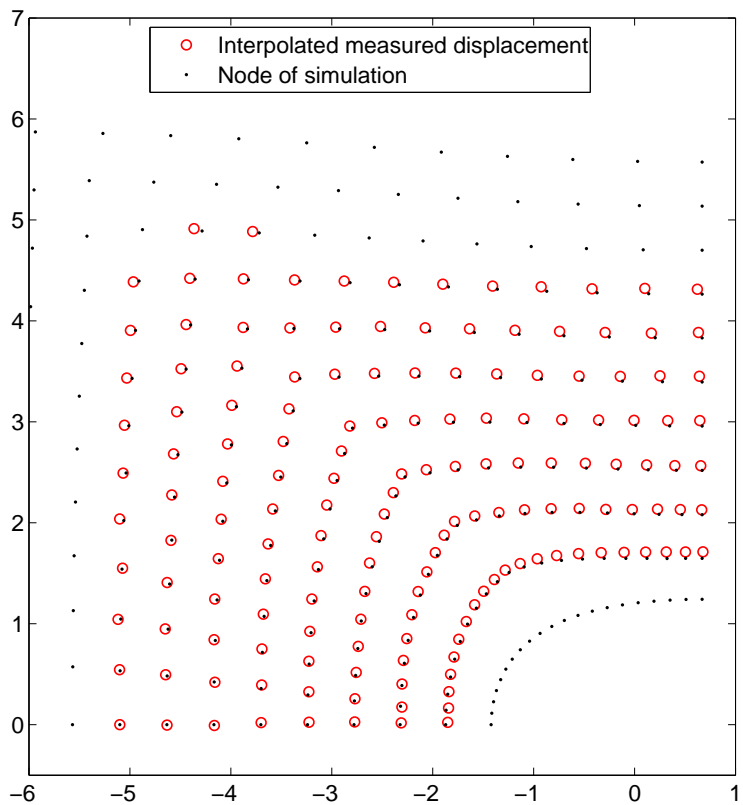

(b) Load step 40 .

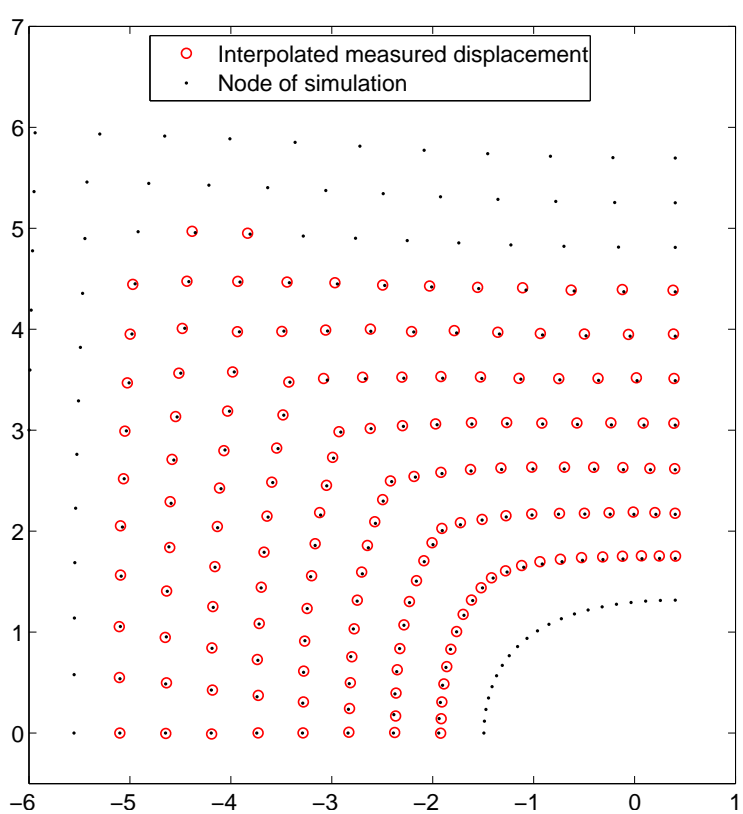

(d) Load step 80 .

Figure 8.12: Verification for test $B(P 84)$ with identified parameters for the combination of the tests $A(P 83), B(P 84)$, and $C(P 85)$; the displacements are multiplied by factor 20 for this representation. 


\section{Verification for test $\mathbf{C}$ (P85)}

Within this verification procedure the simulated data - calculated as based on the optimal parameters $\boldsymbol{\kappa}=\boldsymbol{\kappa}_{l m_{A B C}}$ for the combination of the tests $A(P 83), B(P 84)$, and $C(P 85)$ - is compared with the experimental data for test $C(P 85)$. Figures 8.13-8.14 show the curves with (relative) displacement vs. load steps and force vs. (relative) displacement for the experiment and the simulation.
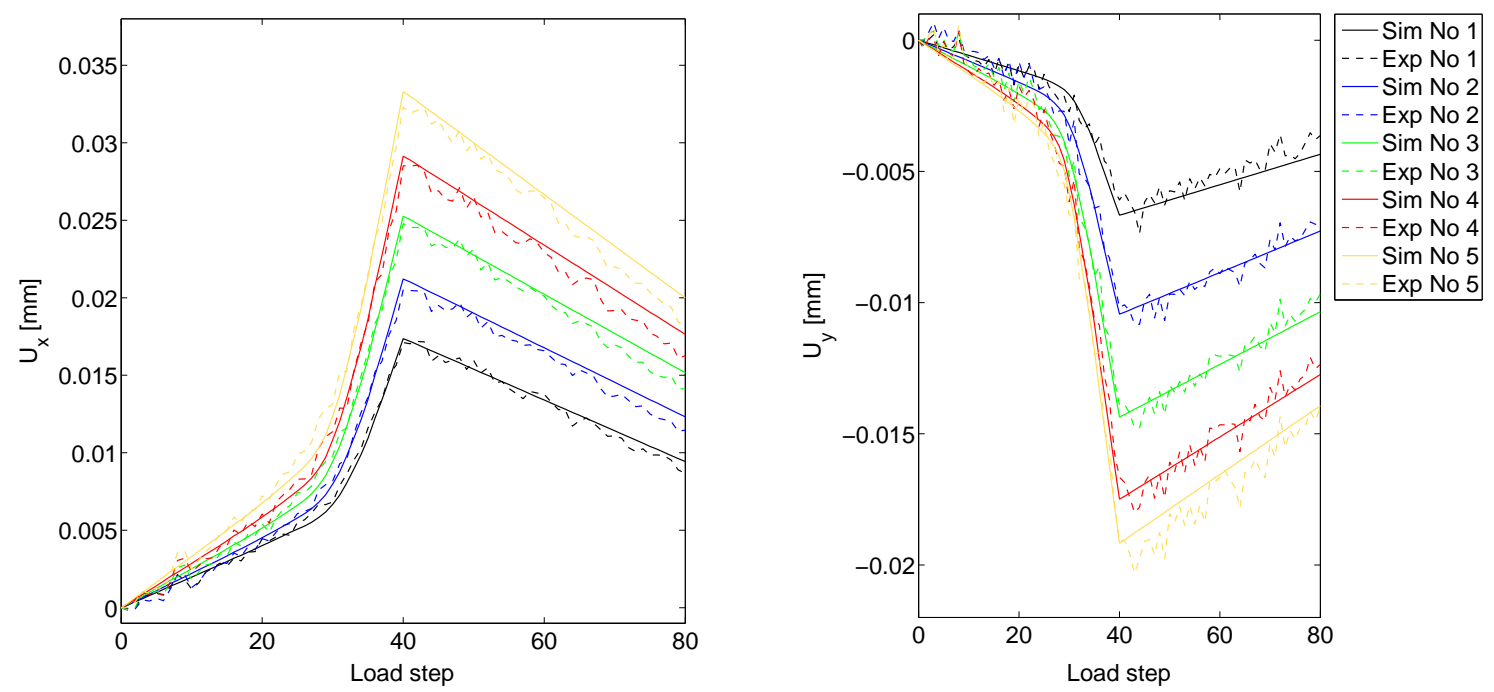

Figure 8.13: Verification for test $C$ with identified parameters for the combination of the tests $A, B, C$.
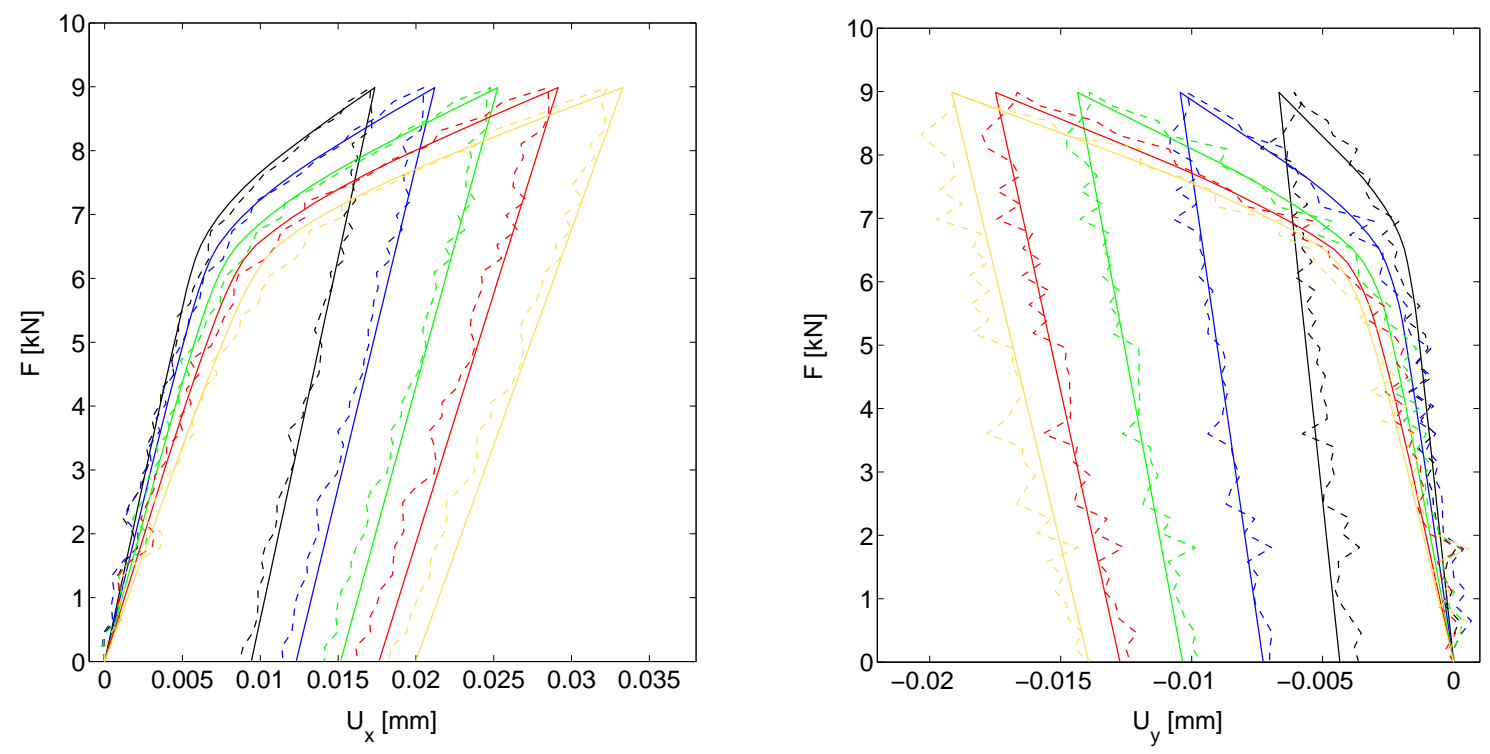

Figure 8.14: Verification for test $C$ with identified parameters for the combination of the tests $A, B, C$.

In figure 8.15 for all identification nodes the simulated positions for the load steps 20, 40, 60 , and 80 are plotted over the interpolated measured displacements for the FE model. For the representation in figure 8.15 the respective displacements are multiplied by factor 20. 


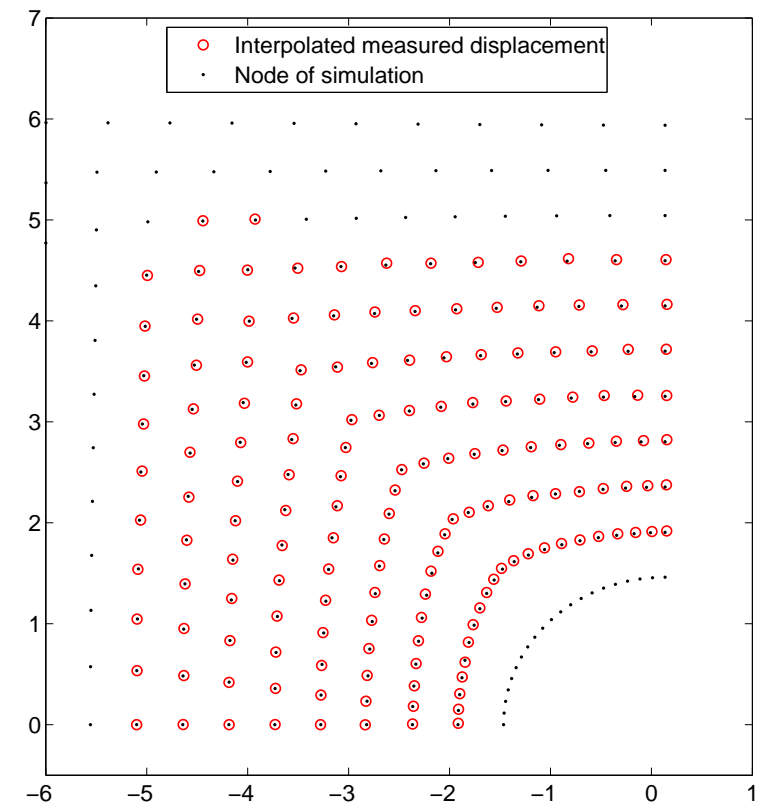

(a) Load step 20 .

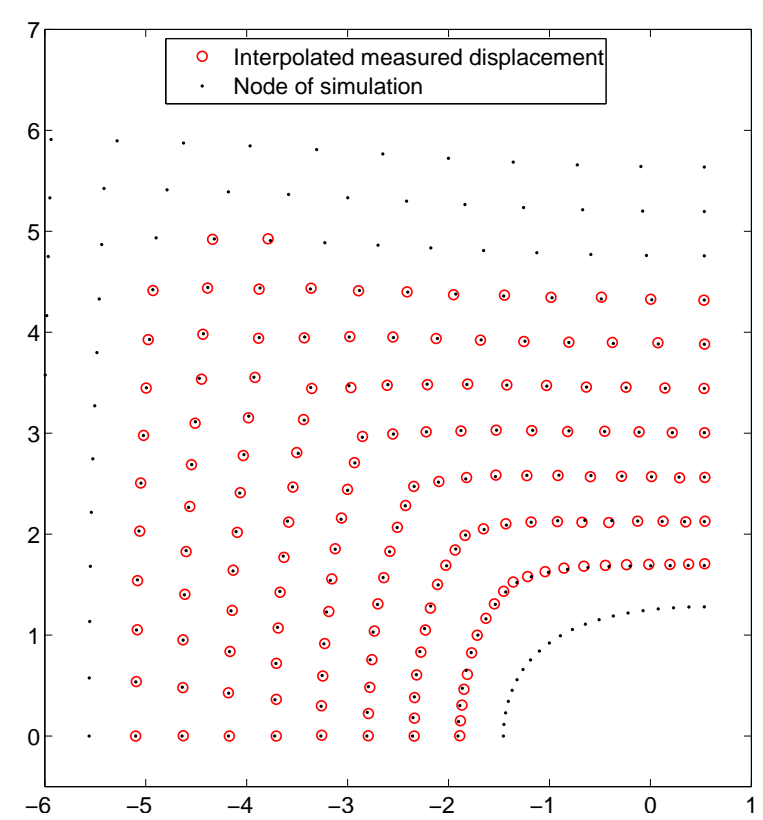

(c) Load step 60 .

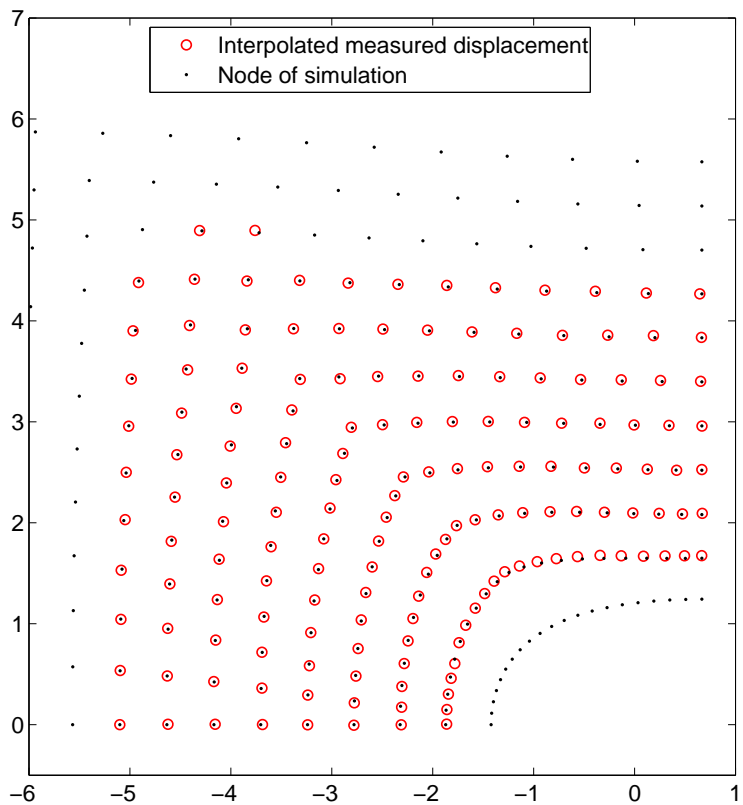

(b) Load step 40 .

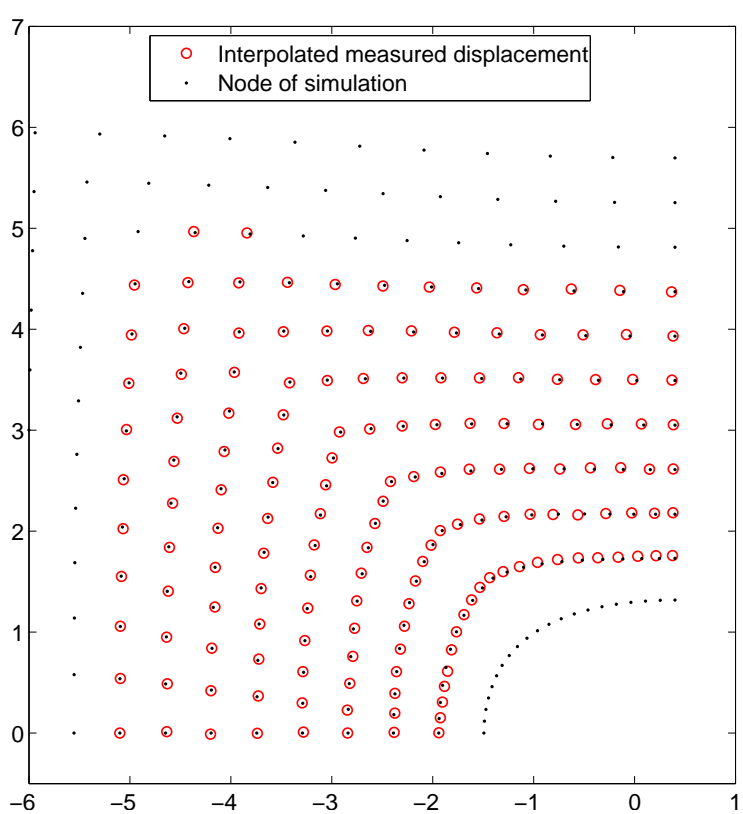

(d) Load step 80 .

Figure 8.15: Verification for test $C(P 85)$ with identified parameters for the combination of the tests $A(P 83), B(P 84)$, and $C(P 85)$; the displacements are multiplied by factor 20 for this representation. 


\subsubsection{Individual identification for P83}

As already mentioned, by comparison of the different sets of parameters for the respective identifications it is investigated, how far the identification routine for the simultaneous identification for all three tests has to average the parameters.

For the individual identification for the test $A(P 83)$ the weighted least squares sum in analogy to equation (8.7) with displacements relative to the identification node $I_{r e l}$ is employed with

$$
f^{A}(\boldsymbol{\kappa})=\frac{1}{2} \sum_{i=1}^{N_{g}} \underbrace{\sum_{j=1}^{T_{A}}\left[\boldsymbol{W}_{i j} \cdot\left[\left(\overline{\boldsymbol{u}}_{i_{r} j}(\boldsymbol{\kappa})-\overline{\boldsymbol{u}}_{i j}(\boldsymbol{\kappa})\right)-\left(\overline{\boldsymbol{u}}_{i_{r} j}^{\exp }-\overline{\boldsymbol{u}}_{i j}^{\exp }\right)\right]\right]^{2}}_{A(P 83)} .
$$

Thereby, $\boldsymbol{W}_{i j}=\operatorname{diag}(1,1)$ are the weighting matrices as described for equation (8.7). For the simulation of the displacement fields $\overline{\boldsymbol{u}}_{i j}(\boldsymbol{\kappa})$ the FEM simulation is force-controlled based on the measured force signals shown in figure 8.3. The number of load steps is $T_{A}=80$ and the number of identification nodes is $N_{g}=159$.

The smallest of all investigated local minima in $\boldsymbol{\kappa}=\boldsymbol{\kappa}_{l m_{A}}$ is shown in table 8.4.

Table 8.4: Parameter set $\kappa=\kappa_{l m_{A}}$.

\begin{tabular}{|l|l|l|l|l|l|}
\hline$E[\mathrm{MPa}]$ & $\nu$ & $y_{0}[\mathrm{MPa}]$ & $y_{\infty}[\mathrm{MPa}]$ & $\omega$ & $H[\mathrm{MPa}]$ \\
\hline \hline 74481.7747 & 0.3257 & 132.4225 & 161.1281 & 225.8283 & 7055.9503 \\
\hline
\end{tabular}

The correlation matrix for the set of optimal parameters for the single identification for test $A(P 83)$ is shown in table 8.5 .

Table 8.5: Correlation matrix for the set of optimal parameters for the single identification for test $A(P 83)$.

\begin{tabular}{|r||l|l|l|l|l|l|}
\hline & $E$ & $\nu$ & $y_{0}$ & $y_{\infty}$ & $\omega$ & $H$ \\
\hline \hline$E$ & 1.000 & 0.238 & -0.383 & 0.133 & -0.074 & -0.163 \\
\hline$\nu$ & 0.238 & 1.000 & -0.144 & 0.054 & 0.007 & -0.089 \\
\hline$y_{0}$ & -0.383 & -0.144 & 1.000 & 0.101 & -0.368 & 0.063 \\
\hline$y_{\infty}$ & 0.133 & 0.054 & 0.101 & 1.000 & -0.953 & -0.981 \\
\hline$\omega$ & -0.074 & 0.007 & -0.368 & -0.953 & 1.000 & 0.877 \\
\hline$H$ & -0.163 & -0.089 & 0.063 & -0.981 & 0.877 & 1.000 \\
\hline
\end{tabular}

For the conclusions and information about the sum of squared differences for the above parameter identification see subsection 8.6.5. 


\section{Verification}

Within this verification procedure the simulated data - calculated as based on the optimal parameters $\boldsymbol{\kappa}=\boldsymbol{\kappa}_{l m_{A}}$ for the single test $A$ - is compared with the experimental data for test $A$. Figures 8.16-8.17 show the curves with (relative) displacement vs. load steps and force vs. (relative) displacement for the experiment and the simulation.
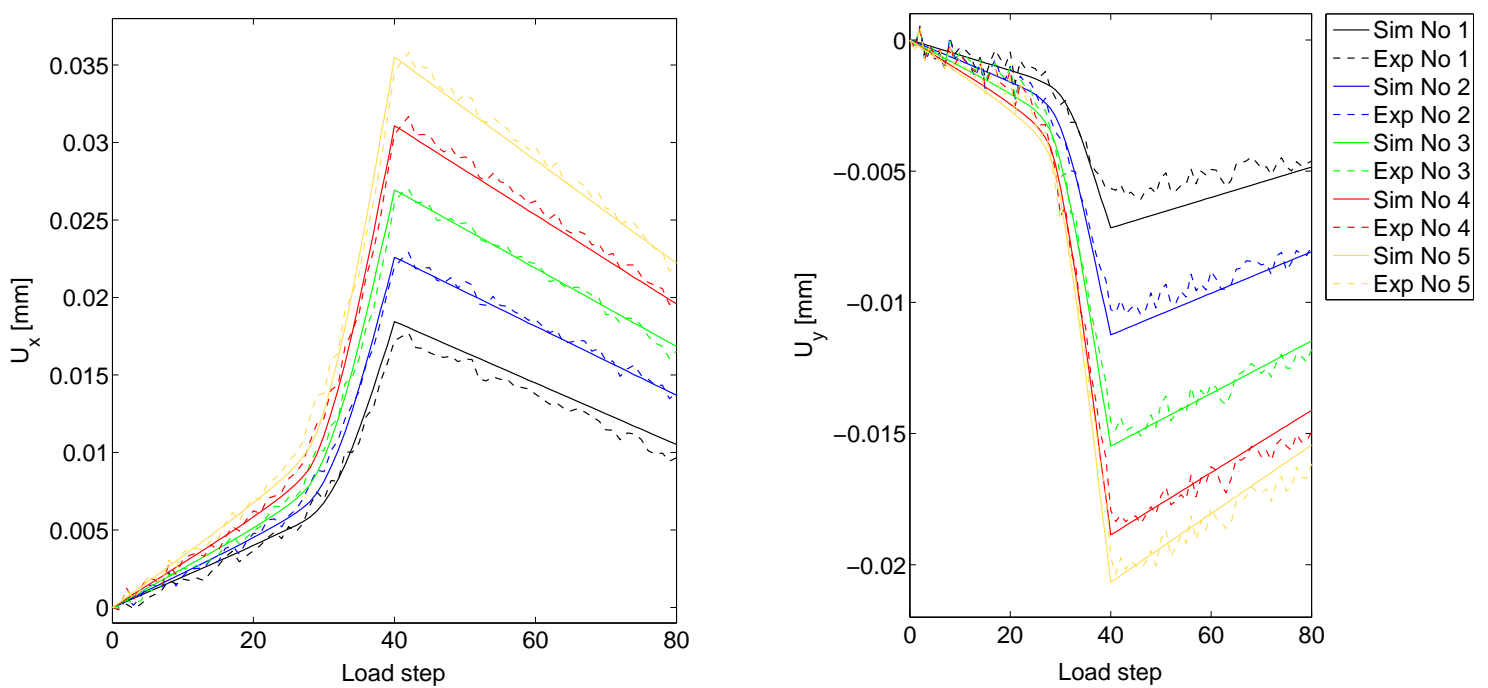

Figure 8.16: Verification for test $A(P 83)$ with identified parameters for the single test $A(P 83)$.
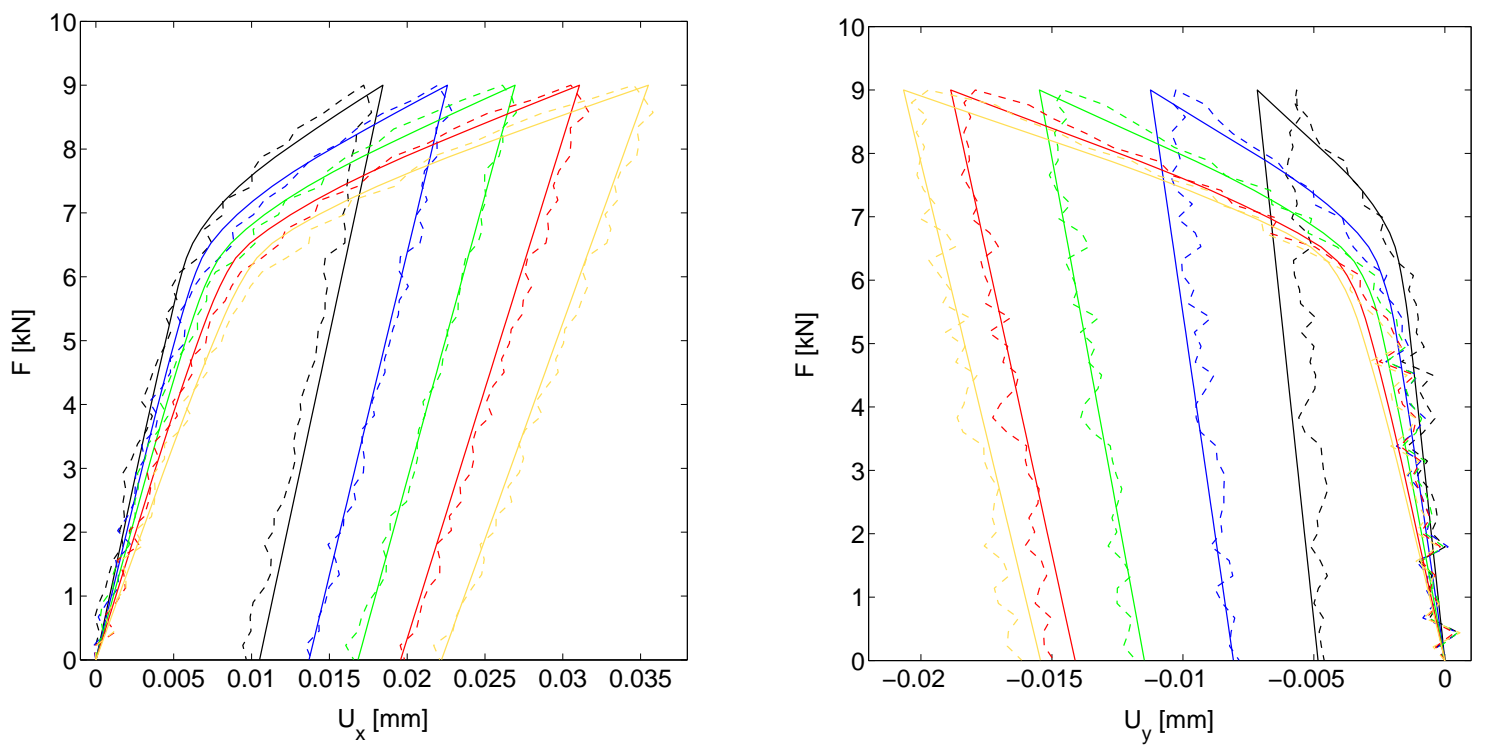

Figure 8.17: Verification for test $A(P 83)$ with identified parameters for the single test $A(P 83)$.

In figure 8.18 for all identification nodes the simulated positions for the load steps 20, 40, 60 , and 80 are plotted over the interpolated measured displacements for the FE model. For the representation in figure 8.18 the respective displacements are multiplied by factor 20. 


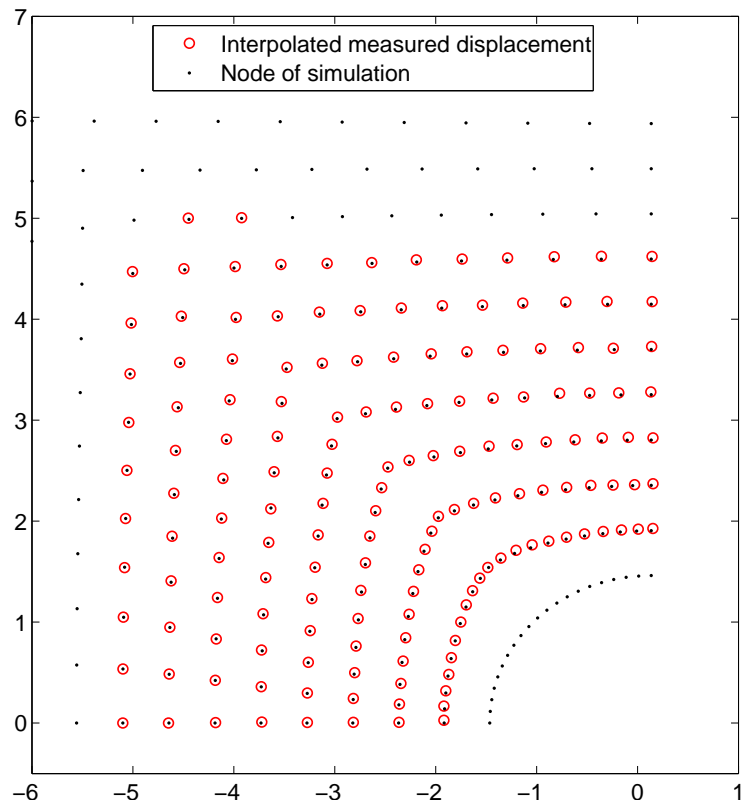

(a) Load step 20.

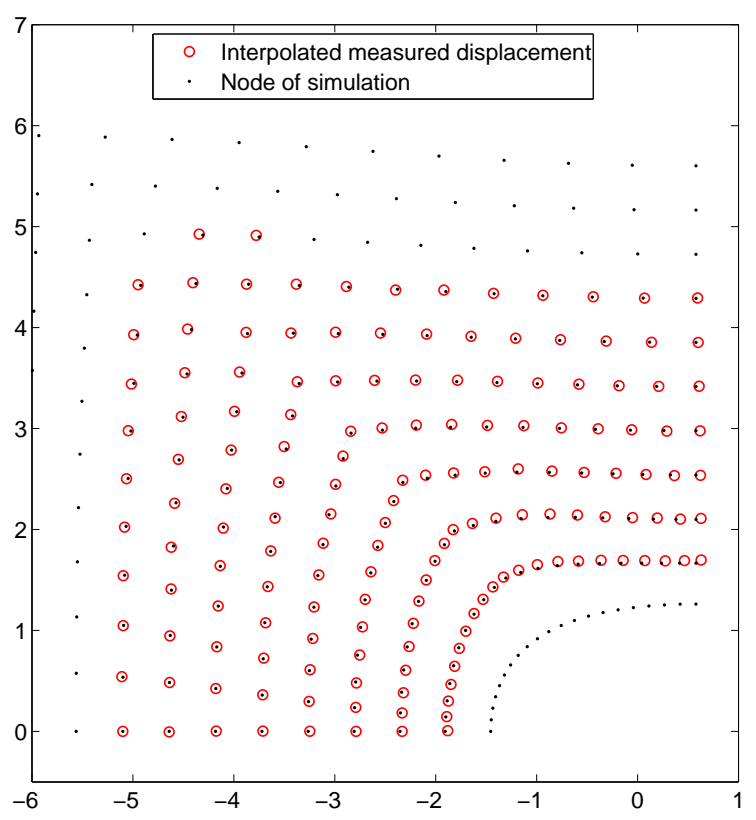

(c) Load step 60 .

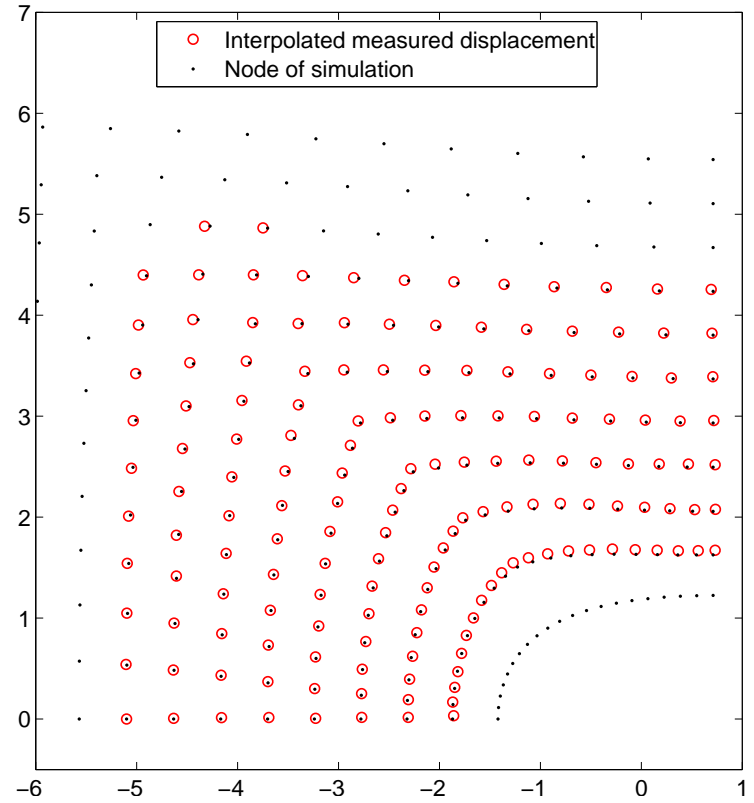

(b) Load step 40.

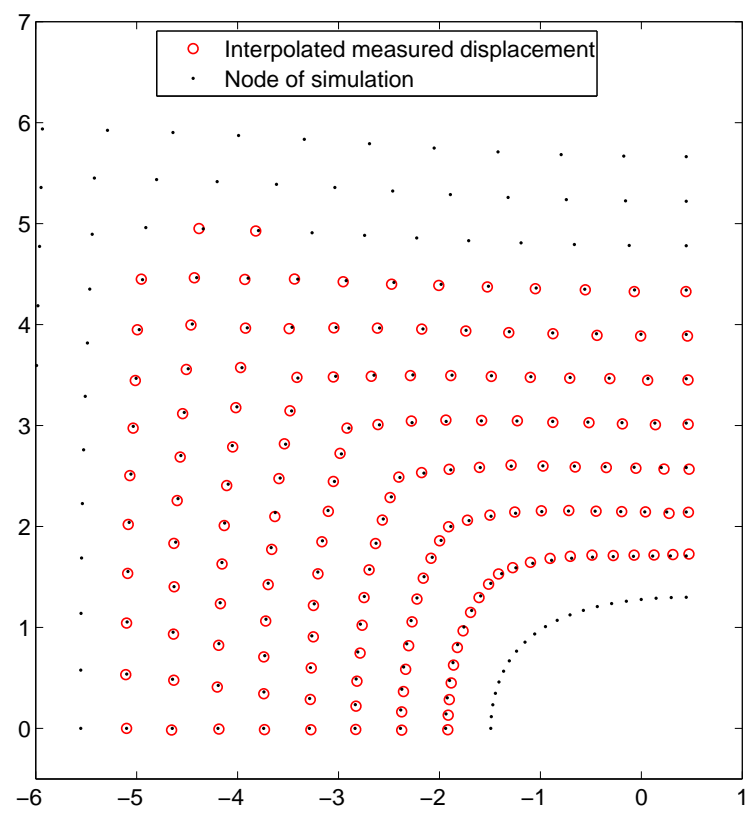

(d) Load step 80 .

Figure 8.18: Verification for single identification for test $A(P 83)$; the displacements are multiplied by factor 20 for this representation. 


\subsubsection{Individual identification for P84}

The weighted least squares sum in analogy to equation (8.7) with displacements relative to the identification node $I_{r e l}$ is employed whereby within the iteration algorithm the test $B(P 84)$ is considered.

$$
f^{B}(\boldsymbol{\kappa})=\frac{1}{2} \sum_{i=1}^{N_{g}} \underbrace{\sum_{k=1}^{T_{B}}\left[\boldsymbol{W}_{i k} \cdot\left[\left(\overline{\boldsymbol{u}}_{i_{r} k}(\boldsymbol{\kappa})-\overline{\boldsymbol{u}}_{i k}(\boldsymbol{\kappa})\right)-\left(\overline{\boldsymbol{u}}_{i_{r} k}^{\text {exp }}-\overline{\boldsymbol{u}}_{i k}^{\text {exp }}\right)\right]\right]^{2}}_{B(P 84)}
$$

$\boldsymbol{W}_{i k}=\operatorname{diag}(1,1)$ are the weighting matrices as described for equation (8.7). For the simulation of the displacement fields $\overline{\boldsymbol{u}}_{i k}(\boldsymbol{\kappa})$ the FEM simulation is force-controlled based on the measured force signals shown in figure 8.3. The number of load steps is $T_{B}=80$ and the number of identification nodes is $N_{g}=159$.

The smallest of all investigated local minima in $\boldsymbol{\kappa}=\boldsymbol{\kappa}_{l_{m_{B}}}$ is shown in table 8.6.

Table 8.6: Parameter set $\kappa=\kappa_{l m_{B}}$.

\begin{tabular}{|l|l|l|l|l|l|}
\hline$E[\mathrm{MPa}]$ & $\nu$ & $y_{0}[\mathrm{MPa}]$ & $y_{\infty}[\mathrm{MPa}]$ & $\omega$ & $H[\mathrm{MPa}]$ \\
\hline \hline 75931.0840 & 0.3132 & 132.9524 & 171.9618 & 225.3120 & 7185.1660 \\
\hline
\end{tabular}

The correlation matrix for the set of optimal parameters for the single identification for test $B(P 84)$ is shown in table 8.7 .

Table 8.7: Correlation matrix for the set of optimal parameters for the single identification for test $B(P 84)$.

\begin{tabular}{|r||l|l|l|l|l|l|}
\hline & $E$ & $\nu$ & $y_{0}$ & $y_{\infty}$ & $\omega$ & $H$ \\
\hline \hline$E$ & 1.000 & 0.242 & -0.431 & 0.113 & -0.004 & -0.157 \\
\hline$\nu$ & 0.242 & 1.000 & -0.138 & 0.088 & 0.011 & -0.133 \\
\hline$y_{0}$ & -0.431 & -0.138 & 1.000 & -0.161 & -0.304 & 0.391 \\
\hline$y_{\infty}$ & 0.113 & 0.088 & -0.161 & 1.000 & -0.863 & -0.958 \\
\hline$\omega$ & -0.004 & 0.011 & -0.304 & -0.863 & 1.000 & 0.683 \\
\hline$H$ & -0.157 & -0.133 & 0.391 & -0.958 & 0.683 & 1.000 \\
\hline
\end{tabular}

For the conclusions and information about the sum of squared differences for the above parameter identification see subsection 8.6.5. 


\section{Verification}

Within this verification procedure the simulated data - calculated as based on the optimal parameters $\boldsymbol{\kappa}=\boldsymbol{\kappa}_{l m_{B}}$ for the single test $B$ - is compared with the experimental data for test $B$. Figures 8.19-8.20 show the curves with (relative) displacement vs. load steps and force vs. (relative) displacement for the experiment and the simulation.
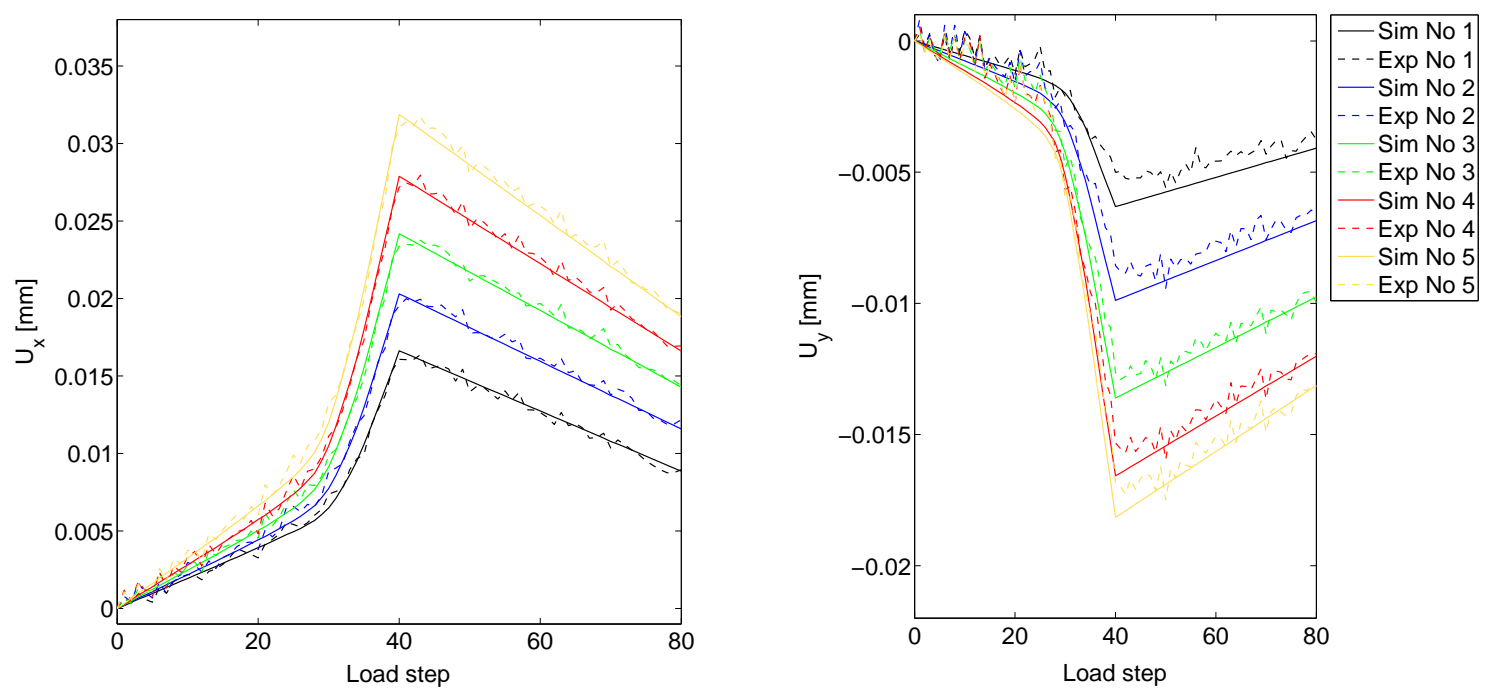

Figure 8.19: Verification for test $B(P 84)$ with identified parameters for the single test $B(P 84)$.
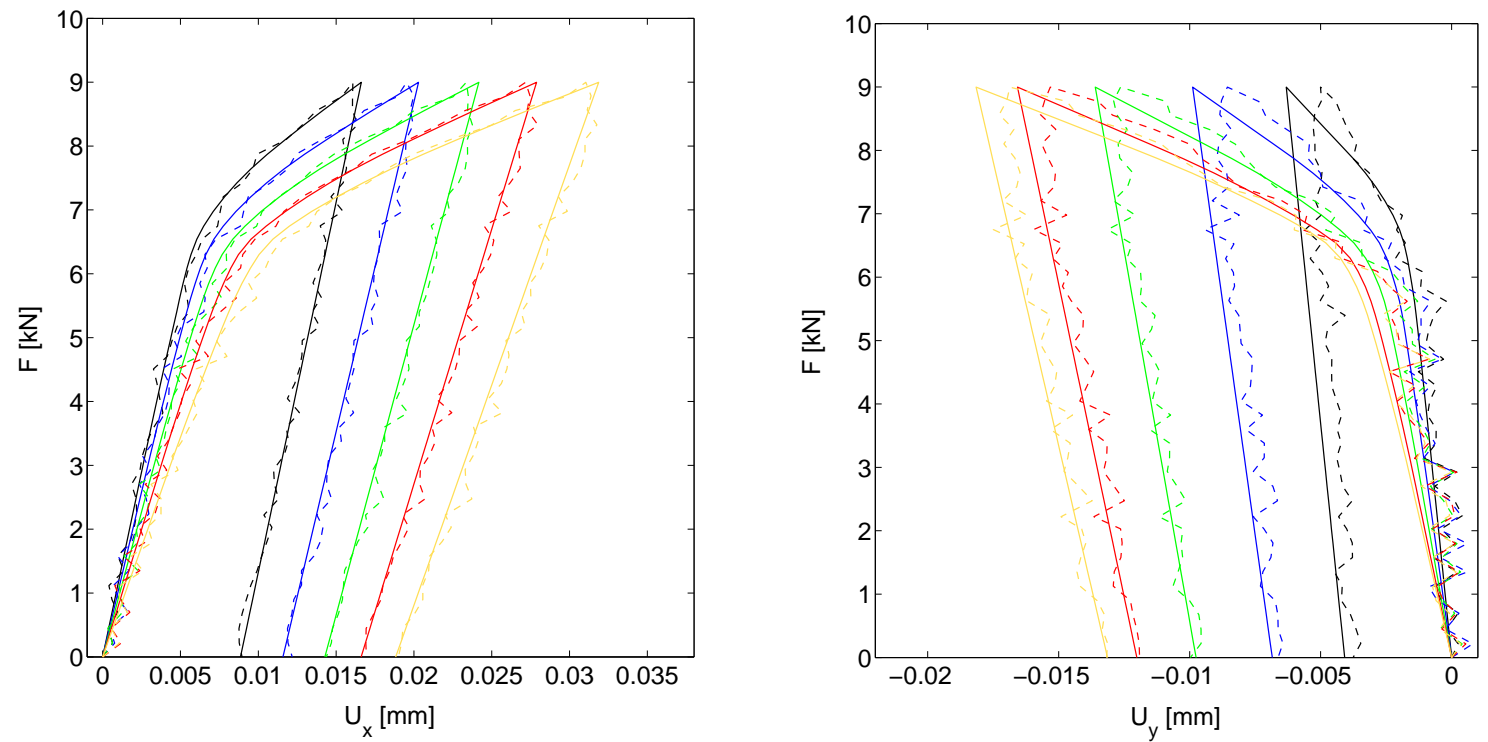

Figure 8.20: Verification for test $B(P 84)$ with identified parameters for the single test $B(P 84)$.

In figure 8.21 for all identification nodes the simulated positions for the load steps 20,40 , 60 , and 80 are plotted over the interpolated measured displacements for the FE model. For the representation in figure 8.21 the respective displacements are multiplied by factor 20. 


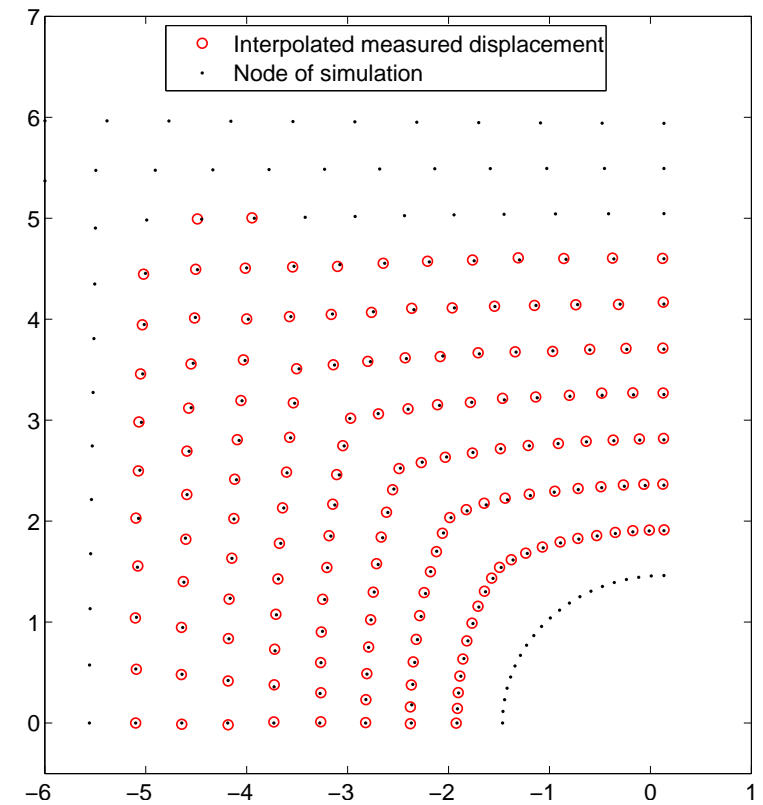

(a) Load step 20 .

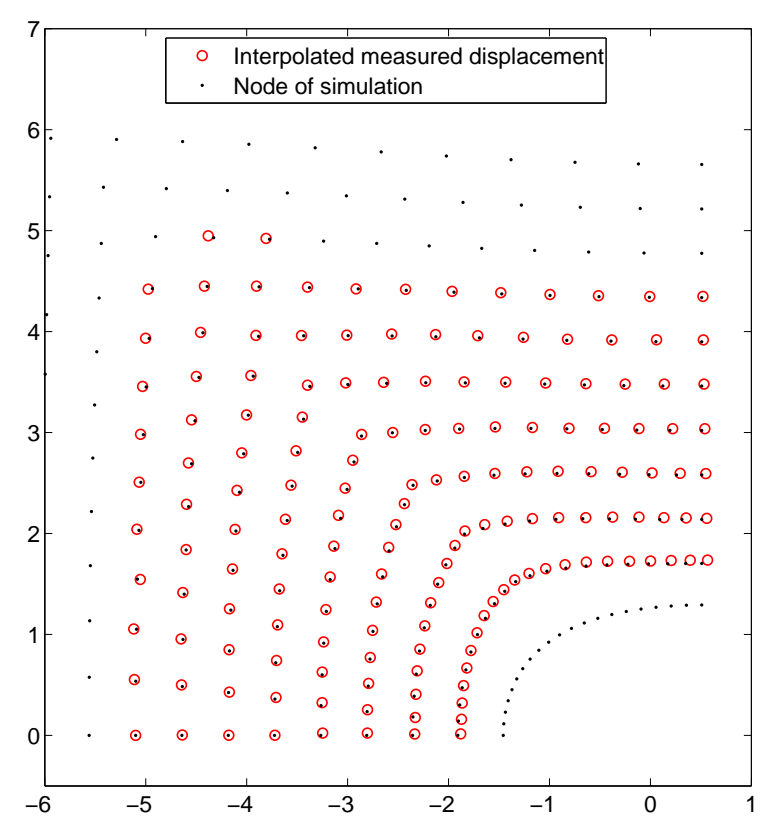

(c) Load step 60 .

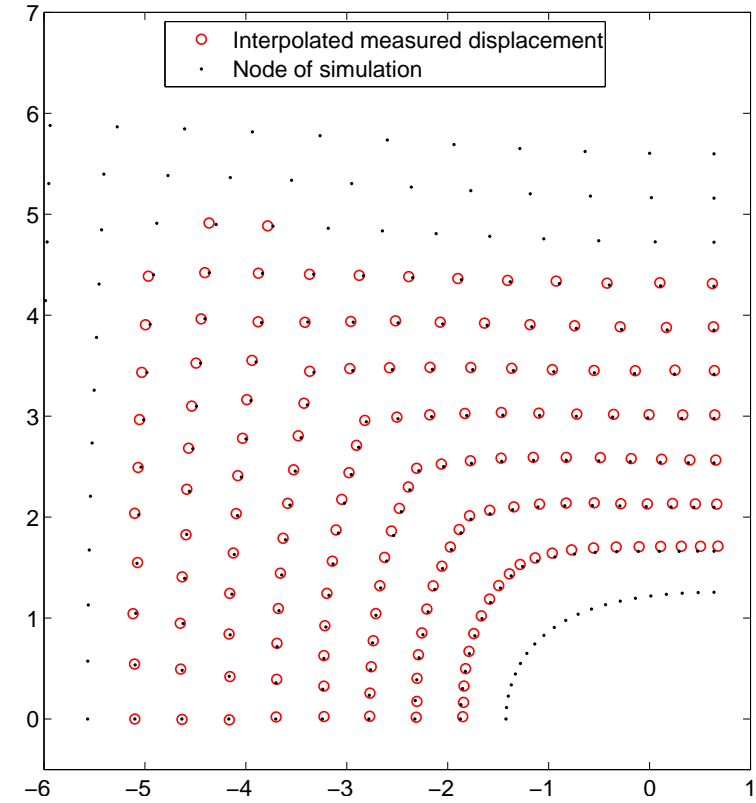

(b) Load step 40 .

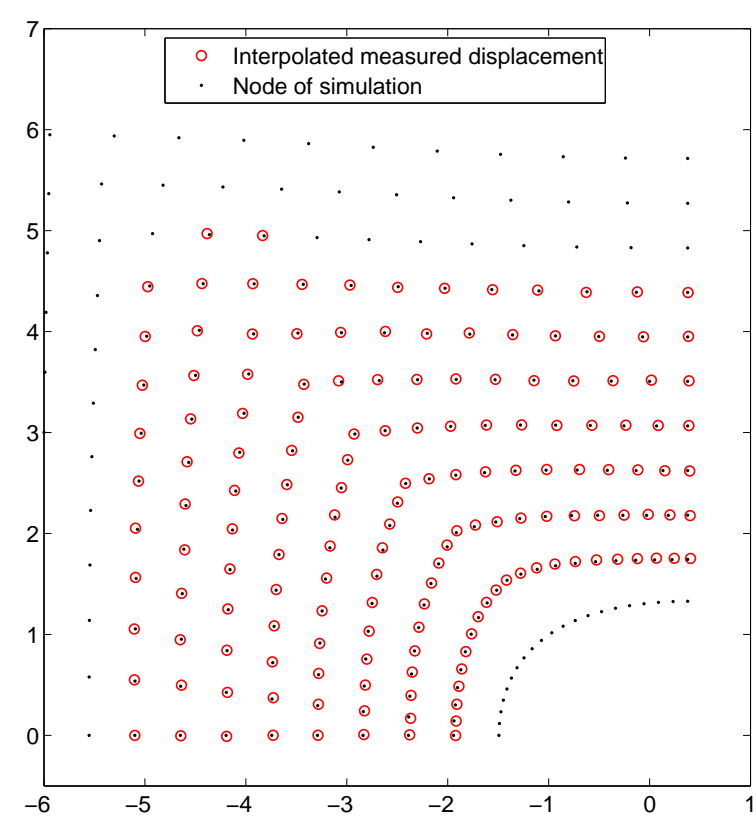

(d) Load step 80 .

Figure 8.21: Verification for single identification for test $B(P 84)$; the displacements are multiplied by factor 20 for this representation. 


\subsubsection{Individual identification for P85}

The weighted least squares sum in analogy to equation (8.7) with displacements relative to the identification node $I_{r e l}$ is employed whereby within the iteration algorithm the test $C(P 85)$ is considered

$$
f^{C}(\boldsymbol{\kappa})=\frac{1}{2} \sum_{i=1}^{N_{g}} \underbrace{\sum_{l=1}^{T_{C}}\left[\boldsymbol{W}_{i l} \cdot\left[\left[\overline{\boldsymbol{u}}_{i_{r} l}(\boldsymbol{\kappa})-\overline{\boldsymbol{u}}_{i l}(\boldsymbol{\kappa})\right]-\left[\overline{\boldsymbol{u}}_{i_{r} l}^{\exp }-\overline{\boldsymbol{u}}_{i l}^{e x p}\right]\right]\right]^{2}}_{C(P 85)} .
$$

$\boldsymbol{W}_{i l}=\operatorname{diag}(1,1)$ are the weighting matrices as described for equation (8.7). For the simulation of the displacement fields $\overline{\boldsymbol{u}}_{i l}(\boldsymbol{\kappa})$ the FEM simulation is force-controlled based on the measured force signals shown in figure 8.3. The number of load steps is $T_{C}=80$ and the number of identification nodes is $N_{g}=159$.

The smallest of all investigated local minima in $\boldsymbol{\kappa}=\boldsymbol{\kappa}_{l_{m_{C}}}$ is shown in table 8.8.

Table 8.8: Parameter set $\kappa=\kappa_{l m_{C}}$.

\begin{tabular}{|l|l|l|l|l|l|}
\hline$E[\mathrm{MPa}]$ & $\nu$ & $y_{0}[\mathrm{MPa}]$ & $y_{\infty}[\mathrm{MPa}]$ & $\omega$ & $H[\mathrm{MPa}]$ \\
\hline \hline 72013.7838 & 0.3564 & 133.4448 & 170.3632 & 225.3081 & $7180.8752]$ \\
\hline
\end{tabular}

The correlation matrix for the set of optimal parameters for the single identification for test $C(P 85)$ is shown in table 8.9 .

Table 8.9: Correlation matrix for the set of optimal parameters for the single identification for test $C(P 85)$.

\begin{tabular}{|r||l|l|l|l|l|l|}
\hline & $E$ & $\nu$ & $y_{0}$ & $y_{\infty}$ & $\omega$ & $H$ \\
\hline \hline$E$ & 1.000 & 0.244 & -0.335 & 0.025 & 0.006 & -0.045 \\
\hline$\nu$ & 0.244 & 1.000 & -0.079 & 0.065 & -0.037 & -0.084 \\
\hline$y_{0}$ & -0.335 & -0.079 & 1.000 & 0.584 & -0.683 & -0.508 \\
\hline$y_{\infty}$ & 0.025 & 0.065 & 0.584 & 1.000 & -0.990 & -0.995 \\
\hline$\omega$ & 0.006 & -0.037 & -0.683 & -0.990 & 1.000 & 0.969 \\
\hline$H$ & -0.045 & -0.084 & -0.508 & -0.995 & 0.969 & 1.000 \\
\hline
\end{tabular}

For the conclusions and information about the sum of squared differences for the above parameter identification see subsection 8.6.5. 


\section{Verification}

Within this verification procedure the simulated data - calculated as based on the optimal parameters $\boldsymbol{\kappa}=\boldsymbol{\kappa}_{l m_{C}}$ for the single test $C$ - is compared with the experimental data for test $C$. Figures 8.22-8.23 show the curves with (relative) displacement vs. load steps and force vs. (relative) displacement for the experiment and the simulation.
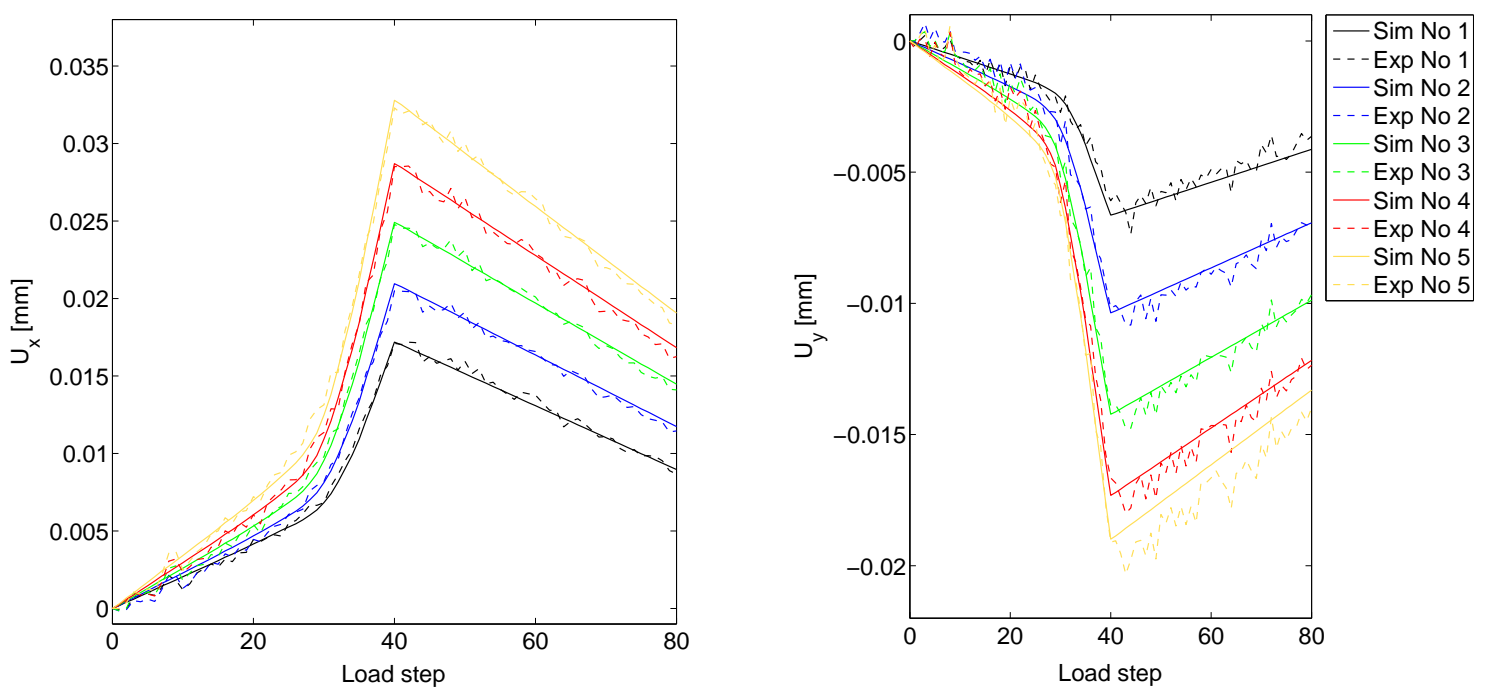

Figure 8.22: Verification for test $C(P 85)$ with identified parameters for the single test $C(P 85)$.
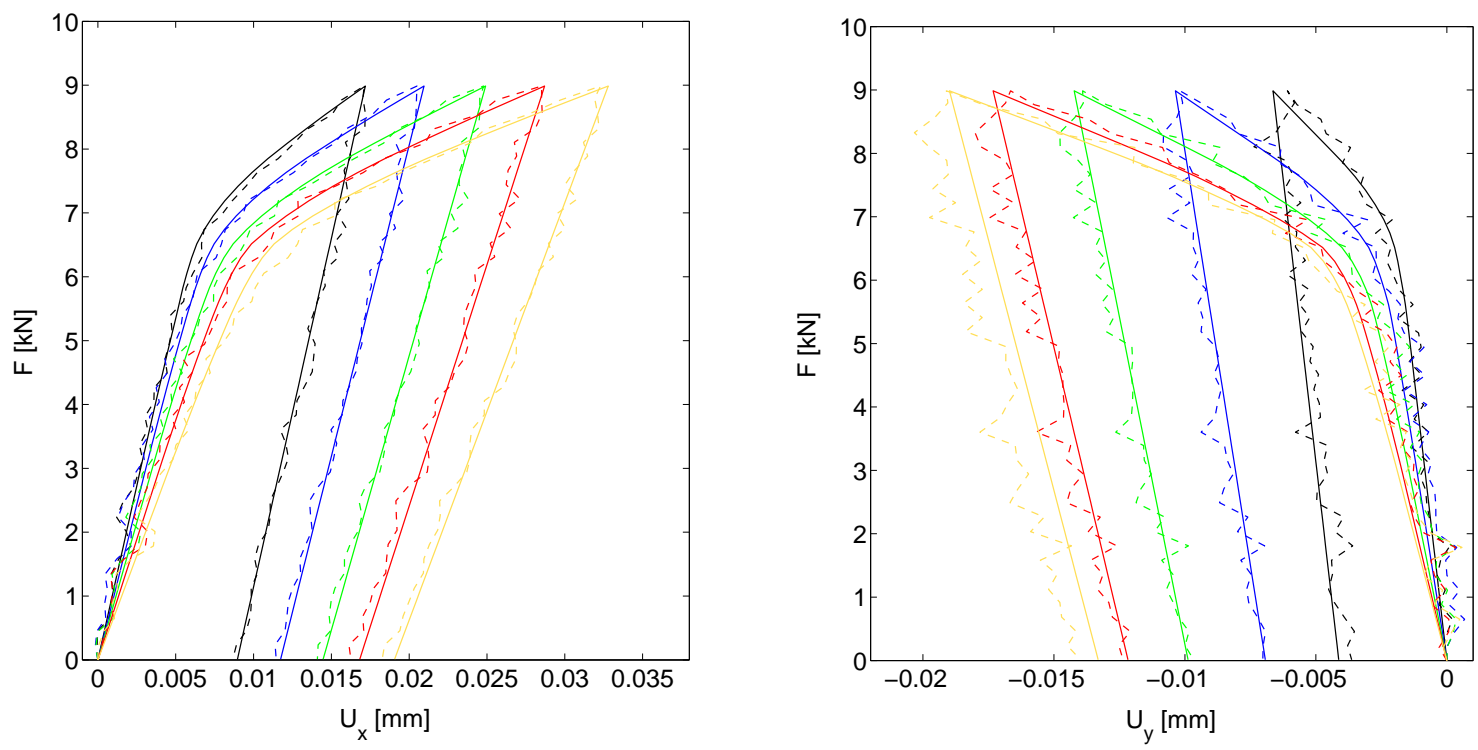

Figure 8.23: Verification for test $C(P 85)$ with identified parameters for the single test $C(P 85)$.

In figure 8.24 for all identification nodes the simulated positions for the load steps 20, 40, 60 , and 80 are plotted over the interpolated measured displacements for the FE model. For the representation in figure 8.24 the respective displacements are multiplied by factor 20 . 


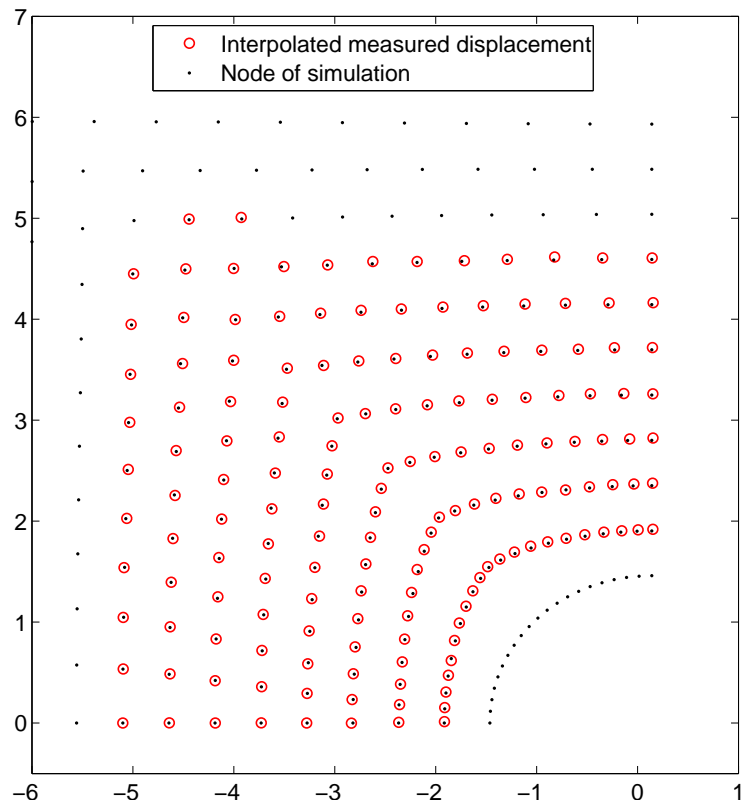

(a) Load step 20.

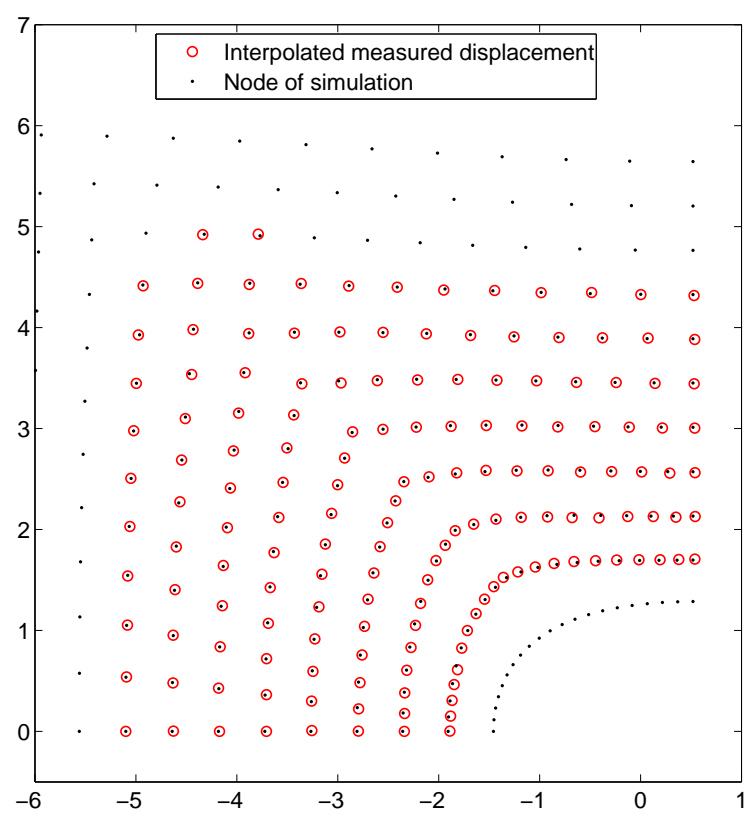

(c) Load step 60 .

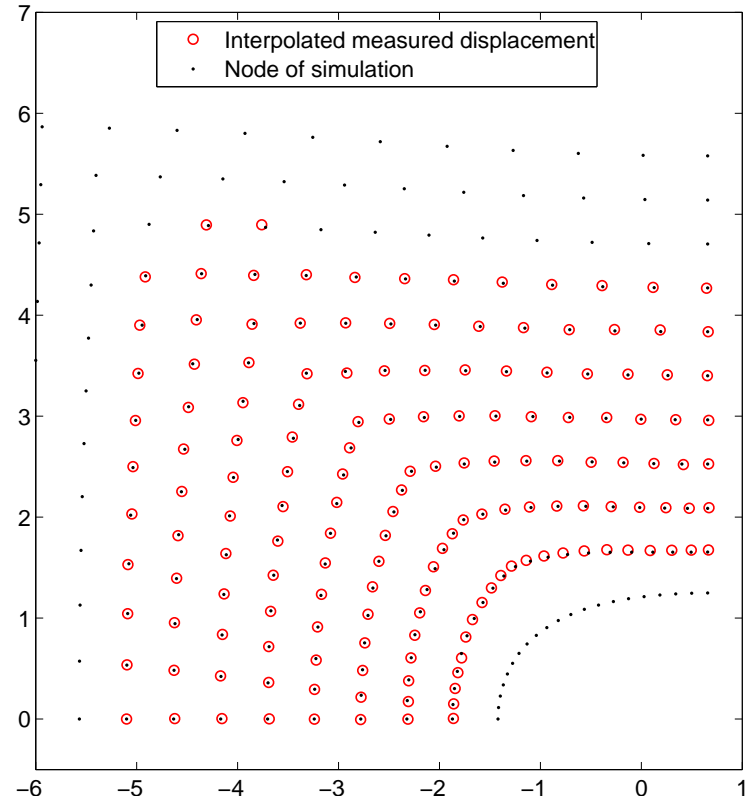

(b) Load step 40.

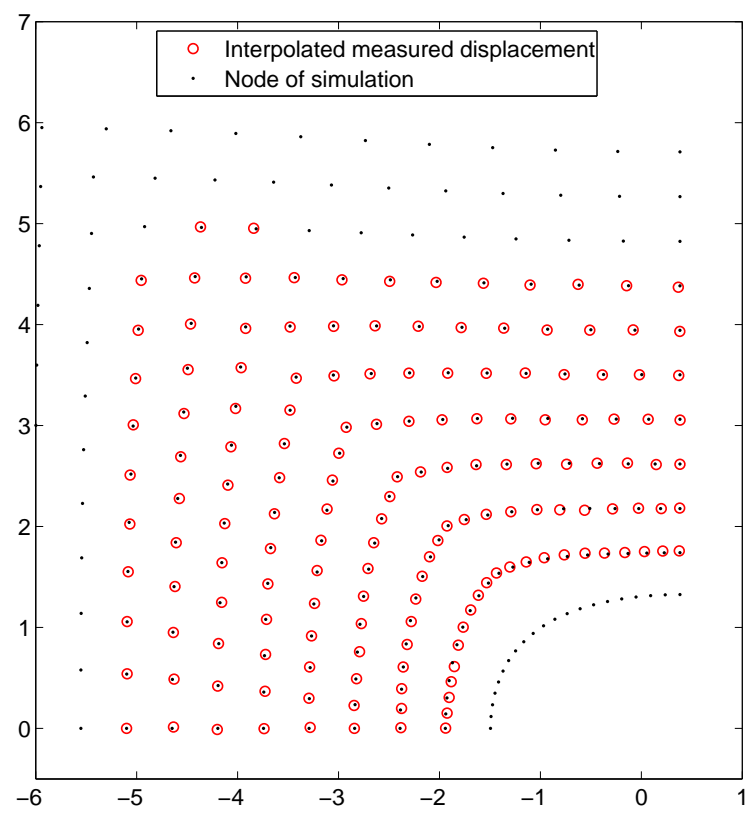

(d) Load step 80 .

Figure 8.24: Verification for single identification for test $C(P 85)$; the displacements are multiplied by factor 20 for this representation. 


\subsubsection{Concluding remarks}

As expected, the verifications for the individual identifications show that the experimental data is in even better agreement with the corresponding simulated data. The comparison of the identified parameter sets is shown in table 8.10. Note that the material parameters

Table 8.10: Comparison of sets of optimal parameters.

\begin{tabular}{|c||l|l|l|l|l|l|}
\hline & $E[\mathrm{MPa}]$ & $\nu$ & $y_{0}[\mathrm{MPa}]$ & $y_{\infty}[\mathrm{MPa}]$ & $\omega$ & $H[\mathrm{MPa}]$ \\
\hline \hline $\boldsymbol{\kappa}_{l m_{A B C}}$ & 74313.9780 & 0.3258 & 132.7606 & 167.0247 & 225.4131 & 7208.3051 \\
\hline \hline $\boldsymbol{\kappa}_{l m_{A}}$ & 74481.7747 & 0.3257 & 132.4225 & 161.1281 & 225.8283 & 7055.9503 \\
\hline $\boldsymbol{\kappa}_{l m_{B}}$ & 75931.0840 & 0.3132 & 132.9524 & 171.9618 & 225.3120 & 7185.1660 \\
\hline $\boldsymbol{\kappa}_{l m_{C}}$ & 72013.7838 & 0.3564 & 133.4448 & 170.3632 & 225.3081 & 7180.8752 \\
\hline $\boldsymbol{\kappa}_{l m_{A, B, C}}^{\varnothing}$ & 74142.2142 & 0.3318 & 132.9399 & 167.8177 & 225.4828 & 7140.6638 \\
\hline
\end{tabular}

in $\boldsymbol{\kappa}_{l m_{A B C}}$ are averaged nonlinearly for the tests $A, B$, and $C$ by means of the least squares algorithm (see equation 8.7). In contrast, the parameter sets of the individual identifications are averaged linearly in $\boldsymbol{\kappa}_{l m_{A, B, C}}^{\varnothing}$. The comparison of the sum of squared differences shown in table 8.11 yields an average sum of squared differences per test for

Table 8.11: Comparison of the sums of squared differences for the sets of optimal parameters.

\begin{tabular}{|c|c|c|c|}
\hline$f^{A B C}\left(\boldsymbol{\kappa}_{l m_{A B C}}\right)\left[\mathrm{mm}^{2}\right]$ & $f^{A}\left(\boldsymbol{\kappa}_{l m_{A}}\right)\left[\mathrm{mm}^{2}\right]$ & $f^{B}\left(\boldsymbol{\kappa}_{l m_{B}}\right)\left[\mathrm{mm}^{2}\right]$ & $f^{C}\left(\boldsymbol{\kappa}_{l m_{C}}\right)\left[\mathrm{mm}^{2}\right]$ \\
\hline \hline 0.0530473 & 0.0121345 & 0.0154719 & 0.00753172 \\
\hline
\end{tabular}

the combination of $A, B$, and $C$ with $\varnothing_{A B C}^{c o m b}=f^{A B C}\left(\boldsymbol{\kappa}_{l m_{A B C}}\right) / 3=0.0176824 \mathrm{~mm}^{2}$ whereas the average sum of squared differences per test for the individual identifications of $A, B$, and $C$ yields $\varnothing_{A B C}^{i n d}=\left[f^{A}\left(\boldsymbol{\kappa}_{l m_{A}}\right)+f^{B}\left(\boldsymbol{\kappa}_{l m_{B}}\right)+f^{C}\left(\boldsymbol{\kappa}_{l m_{C}}\right)\right] / 3=0.0117127 \mathrm{~mm}^{2}$. Hence, the average reduction of the sum of squared differences from the combination to the individual identifications is $\Delta f=100\left(\varnothing_{A B C}^{c o m b}-\varnothing_{A B C}^{i n d}\right) / \varnothing_{A B C}^{c o m b}=66.24 \%$.

Remark 8.6.1 As already mentioned with reference to the correlation matrix shown in table 8.3, the parameters describing the hardening of the material, i.e. $y_{\infty}, \omega$, and $H$ are relatively unstable. Also for the individual identifications these parameters have high correlation numbers and due to this they behave (almost) indifferent. This effect is due to overparametrization whereby the iterations show that mainly the parameter $H$ - although the respective term for linear hardening in the material model contributes to a lowering of the sum of squared differences - is redundant.

In order to determine parameter sets which are comparable to the parameter set $\boldsymbol{\kappa}_{l m_{A B C}}$, for the individual identifications the parameter $H$ has been controlled in the respective identification processes to stay within the limits $7000 \mathrm{MPa} \leq H \leq 7400 \mathrm{MPa}$ in correspondence with the result $H=7208.3051 \mathrm{MPa}$ for $\boldsymbol{\kappa}_{l m_{A B C}}$. Due to the indifferent behavior of $H$ within the iteration processes, this limitation has practically no influence on the sum of least squares. By applying this limitation the respective parameter sets are stable and unique. 
Concluding the reflection of the results of the respective identifications for the three tests, figure 8.25 shows the stress vs. strain curves for uniaxial tension for the parameter sets $\boldsymbol{\kappa}_{l m_{A B C}}, \boldsymbol{\kappa}_{l m_{A}}, \boldsymbol{\kappa}_{l m_{B}}$, and $\boldsymbol{\kappa}_{l m_{C}}$.

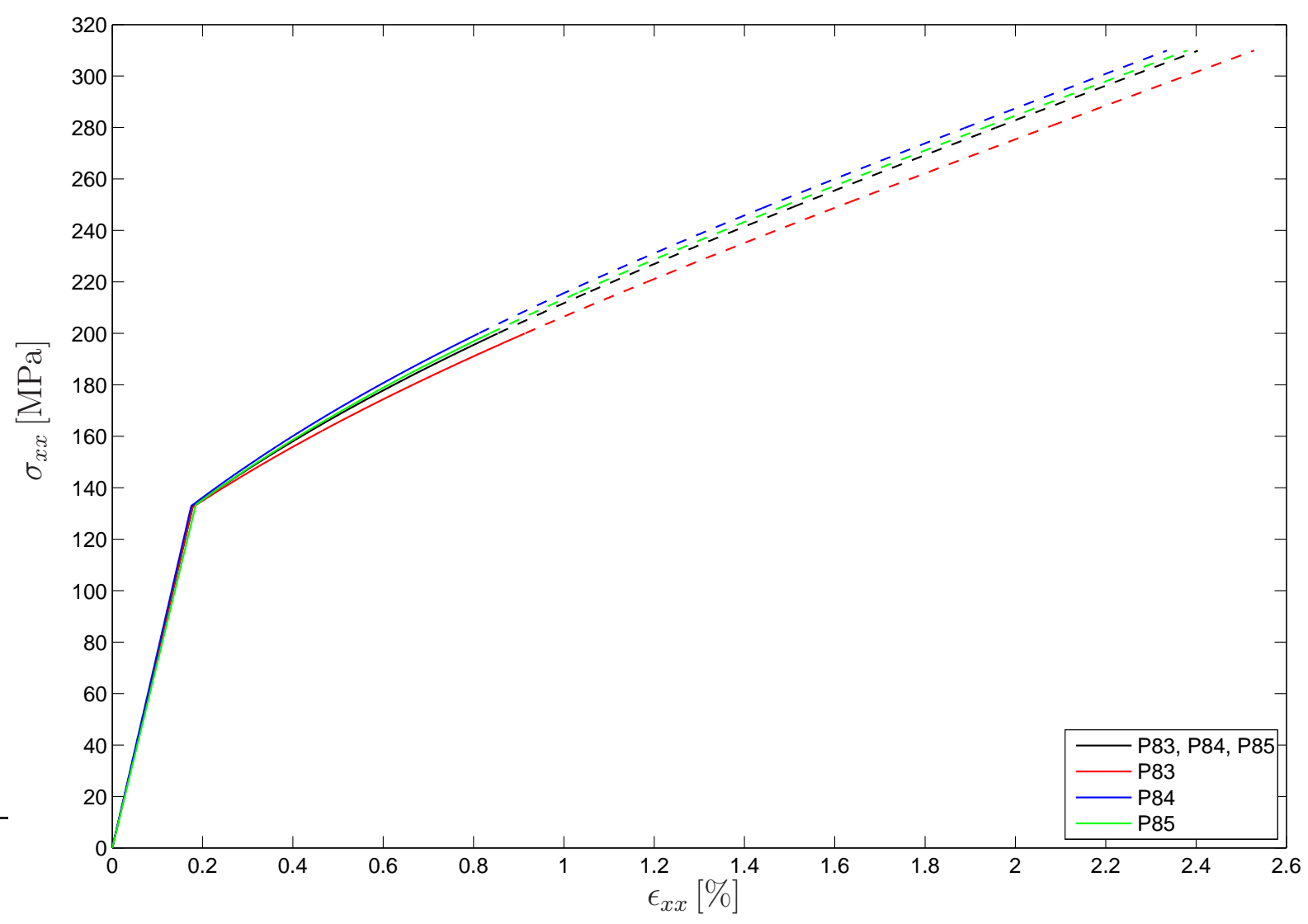

Figure 8.25: Stress vs. strain curves for uniaxial tension for the different identified parameter sets. Black: $\kappa=\kappa_{l m_{A B C}}$; Red: $\kappa=\kappa_{l m_{A}}$; Blue: $\kappa=\kappa_{l m_{B}}$; Green: $\kappa=\kappa_{l m_{C}}$. Continuous lines: proved by experimental data. Dashed lines: extrapolated.

Thereby, for the simulation of the homogeneous tensile tests the uniaxial state of stress at the eight-noded enhanced element is considered (see figure 8.26). The homogeneous tensile tests for the different sets of material parameters underlines, that the scattering of the specimen due to the different material behavior is mainly restricted to a different mechanical behavior within the plastic range of the deformation.

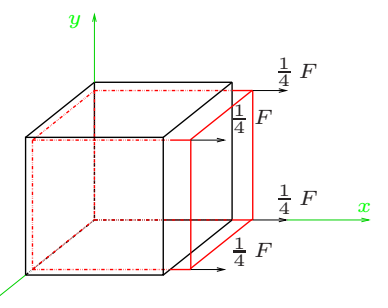

Figure 8.26: Uniaxial state of stress at the eight-node enhanced element (Q1E9). 
Since the three tests are conducted in force control to a maximal force load of $9000 \mathrm{~N}$ (see figure 8.3), at the hole the maximum tensile load per square unit of original cross section yields $200 \mathrm{MPa}$. Therefore, in figure 8.25 the computed stress strain curves have been denominated as 'extrapolated' for $\sigma_{x x}>200 \mathrm{MPa}$. Figure 8.27 shows the FE model of the specimen and the euclidean norm of the Cauchy stresses for test $A(P 83)$ at load step 40 (maximal load) for the optimal set of parameters $\boldsymbol{\kappa}_{l m_{A}}$.

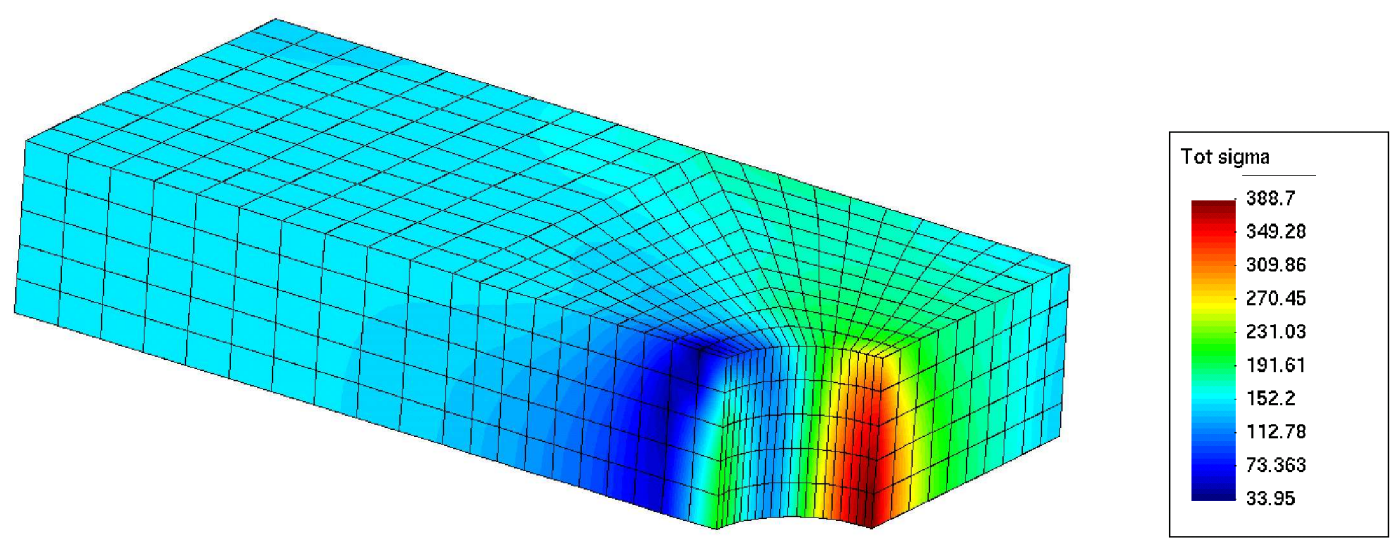

Figure 8.27: Simulation of test $A(P 83)$ at load step 40; Euclidean norm of the Cauchy stresses. 


\section{Summary and conclusions}

An inverse problem is the task where the values of model parameter(s) must be obtained from the observed data. Inverse problems are typically ill-posed, as opposed to the, in general, well-posed direct problems when the model parameters are known. The formulation of an inverse problem in order to establish an appropriate simulation tool for a material under operational conditions involves different disciplines: In experiments the mechanical behavior of the material has to be tested and measured. Therefore, suitable testing machines and accurate measurement systems have to be applied. Using numerical techniques, the constitutive behavior which has been observed in the experiments, has to be modeled mathematically so that a simulation is able to reproduce the experiments properly.

The type of inverse problem to be solved in this work has been to identify the parameters for a model of finite viscoelasticity. This model has been proposed by Reese and Govindjee [65] and has been implemented to a finite-element code. The inverse problem is approximated by an optimization problem, with which we aim at finding the optimal fit of measured and simulated displacement fields. Thereby, often stability problems occur due to the ill-posedness of the inverse problem.

In order to establish a reliable simulation technique for a considered material, appropriate experiments in combination with a sufficient model structure of the viscoelastic constitutive routine have to be chosen. Moreover, since experiments with a purely homogeneous displacement field are difficult to conduct and contain less information than experiments with inhomogeneous displacement fields, the non-uniformity of stresses and strains has to be considered in the identification algorithm (see Mahnken [40]).

The applicability of the identification algorithm for one experiment in combination with the viscoelastic model has been shown on the basis of the parameter identification for virtual measurement data and for displacement fields of a cured polyurethane adhesive.

In order to consider the scattering of the mechanical behavior between different specimen and in addition to identify the parameters to guarantee a higher reliability, multiple tests for different specimen have to be considered. Only the consideration of experimental data, activating all different mechanical processes the constitutive model is able to simulate, can avoid instabilities in the solution. The presented method of identifying all material parameters simultaneously for multiple tests leads to an optimal set of parameters which satisfies all tests similarly well. This has been presented by means of the parameter identification for a cellular polyurethane foam, whereby one relaxation module has been implemented in the constitutive routine.

For an example of a compact polyurethane the viscoelastic routine with two relaxation modules has been implemented and the simultaneous identification of all parameters for the three tests has been carried out. Based on the method the experimental loading intervals for long-time experiments can be shortened in time and the parameter identification 
procedure is now referred to experimental data of tests under short- and long-time loads without separating the parameters due to these different time scales.

Finally the parameter identification for small strain plasticity has been conducted for the example of the aluminum alloy AlSi9Cu3. Thereby it could be shown, that the identification algorithm can be applied to different constitutive laws. Moreover it became obvious that by consideration of multiple tests it is possible to determine nonlinearly an averaged set of material parameters, whereby the measurement fields are scattered due to different material properties of different samples and due to significant noise of the measurement system in combination with uncertainties of the force control of the testing machine.

Generally it could be presented for all examples that the calculation of the parameter correlations and different verifications helps to check the general validity of the identified parameters for the prediction of the constitutive behavior of engineering components. 


\section{A Derivatives}

\section{A.1 Calculation of $\partial\left(\boldsymbol{n}_{\mathrm{a}} \otimes \boldsymbol{n}_{\mathrm{a}}\right) / \partial \boldsymbol{b}$}

In this Appendix, the calculation of $\partial\left(\boldsymbol{n}_{\mathrm{a}} \otimes \boldsymbol{n}_{\mathrm{a}}\right) / \partial \boldsymbol{b}$ is reviewed for convenience of the reader, whereby $\boldsymbol{n}_{\mathrm{a}} \otimes \boldsymbol{n}_{\mathrm{a}}$ is the tensor basis of a positive-definite symmetric second-order tensor $\boldsymbol{b}$. For the proof and further details see e.g. Miehe [56].

The spectral decomposition of $\boldsymbol{b}$ yields the representation

$$
\boldsymbol{b}=\sum_{\mathrm{a}=1}^{3} \lambda_{\mathrm{a}}^{2} \boldsymbol{n}_{\mathrm{a}} \otimes \boldsymbol{n}_{\mathrm{a}}
$$

with $\left\|\boldsymbol{n}_{\mathrm{a}}\right\|=1$ and $\lambda_{\mathrm{a}}^{2}$ denoting the eigenvalues of $\boldsymbol{b}$ such that the third invariant of $\boldsymbol{b}$ is given by $J_{3}(\boldsymbol{b})=\lambda_{1}^{2} \lambda_{2}^{2} \lambda_{3}^{2}$. By introducing

$$
D_{\mathrm{a}}=\prod_{\mathrm{b}=1 \backslash \mathrm{a}}^{3}\left[\lambda_{\mathrm{a}}^{2}-\lambda_{\mathrm{b}}^{2}\right]
$$

we arrive at

$$
\begin{aligned}
\frac{\partial\left(\boldsymbol{n}_{\mathrm{a}} \otimes \boldsymbol{n}_{\mathrm{a}}\right)}{\partial \boldsymbol{b}} & =\frac{\lambda_{\mathrm{a}}^{2}}{D_{\mathrm{a}}}\left[\mathbf{I}_{\boldsymbol{I}}-J_{3} \lambda_{\mathrm{a}}^{-2} \mathbf{I}_{\boldsymbol{b}^{-1}}\right] \\
& +\frac{\lambda_{\mathrm{a}}^{2}}{D_{\mathrm{a}}} \sum_{\mathrm{b}=1}^{3}\left[J_{3} \lambda_{\mathrm{a}}^{-2}-\lambda_{\mathrm{b}}^{4}\right] \lambda_{\mathrm{b}}^{-4} \boldsymbol{n}_{\mathrm{b}} \otimes \boldsymbol{n}_{\mathrm{b}} \otimes \boldsymbol{n}_{\mathrm{b}} \otimes \boldsymbol{n}_{\mathrm{b}}
\end{aligned}
$$

wherein $\mathbf{I}_{\boldsymbol{I}}$ is the fourth-order identity tensor with the components $\left[\mathbf{I}_{\boldsymbol{I}}\right]^{I J K L}=\frac{1}{2}\left[[\boldsymbol{I}]^{I K}[\boldsymbol{I}]^{J L}+[\boldsymbol{I}]^{I L}[\boldsymbol{I}]^{J K}\right]$. The fourth-order tensor $\mathbf{I}_{\boldsymbol{b}^{-1}}=-\partial \boldsymbol{b}^{-1} / \partial \boldsymbol{b}$ has the components $\left[\mathbf{I}_{\boldsymbol{b}^{-1}}\right]^{I J K L}=\frac{1}{2}\left[\left[\boldsymbol{b}^{-1}\right]^{I K}\left[\boldsymbol{b}^{-1}\right]^{J L}+\left[\boldsymbol{b}^{-1}\right]^{I L}\left[\boldsymbol{b}^{-1}\right]^{J K}\right]$. 


\section{Bibliography}

[1] K. Andresen, S. Dannemeyer, H. Friebe, R. Mahnken, R. Ritter, and E. Stein. Parameteridentifikation für ein plastisches Stoffgesetz mit FE-Methoden und Rasterverfahren. Der Bauingenieur, 71:21-31, 1996.

[2] H.T. Banks and K. Kunisch. Estimation Techniques for Distributed Parameter Systems. Birkhäuser, 1989.

[3] Y. Bard. Nonlinear Parameter Estimation. Academic Press, 1974.

[4] J. Baumeister. Stable Solution of Inverse Problems. Vieweg \& Sohn, 1987.

[5] M. Bazaraa, H. Sherali, and C. Shetty. Nonlinear Programming. Wiley, 1993.

[6] G.W. Becker, D. Braun, and G. Oertel. Polyurethane, volume 7 of Kunststoff Handbuch. Hanser, 1993.

[7] D.P. Bertsekas. Nonlinear Programming. Athena Scientific, 1999.

[8] J. Betten. Kontinuumsmechanik. Springer, 1993.

[9] Markus Böl. Numerische Simulation von Polymernetzwerken mit Hilfe der FiniteElemente-Methode. Phd thesis, Institut für Mechanik, Ruhr-Universität Bochum, 2005. Mitteilungen aus dem Institut für Mechanik Nr. 135.

[10] J. Bonet and R.D. Wood. Nonlinear Continuum Mechanics for Finite Element Analysis. Cambridge University Press, 1997.

[11] M. Bosseler, B. Kleuter, R. Renz, and P. Steinmann. Experiment and parameter identification for the simulation of compact and cellular pur. Kautschuk Gummi Kunststoffe, 2007. accepted for publication.

[12] R.P. Brown. Physical Testing of Rubber. Chapman \& Hall, 1996.

[13] H.D. Bui. Inverse Problems in the Mechanics of Materials, an Introduction. CRC Press, 1994.

[14] J.E. Dennis and R.B. Schnabel. Numerical Methods for Unconstrained Optimization and Nonlinear Equations. Prentice Hall, 1983.

[15] M. Ekh. Thermo-elastic--viscoplastic modeling of in792. J. Mech. Behav. Mater., 12:359-387, 2001.

[16] A.C. Eringen. Mechanics of Continua. Krieger, 2nd edition, 1989. 
[17] W.N. Findley, J.S. Lai, and K. Onaran. Creep and Relaxation of Nonlinear Viscoelastic Materials; With an Introduction to Linear Viscoelasticity. North Holland, 1976.

[18] S. Govindjee and S. Reese. A presentation and comparison of two large deformation viscoelasticity models. ASME J. Eng. Mat. Tech., 119:251-255, 1997.

[19] S. Govindjee and J.C. Simo. Mullins effects and the strain amplitude dependence of the elastic modulus. Int. J. Solids Struct., 29:1737-1751, 1992.

[20] S. Hartmann, T. Tschöpe, L. Schreiber, and P. Haupt. Finite deformations of a carbon black-filled rubber. experiment, optical measurement and material parameter identification using finite elements. Euro. J. Mech. A/Solids, 22:309-324, 2003.

[21] P. Haupt. Continuum Mechanics and Theory of Materials. Springer, 2000.

[22] P. Haupt and K. Sedlan. Viscoplasticity of elastomeric materials: experimental facts and constitutive modelling. Arch. Appl. Mech., 71:89-109, 2001.

[23] G.A. Holzapfel. Nonlinear Solid Mechanics. Wiley, 2000.

[24] G.A. Holzapfel and J.C. Simo. A new viscoelastic constitutive model for continuous media at finite thermodynamical changes. Int. J. Solids Struct., 33:3019-3034, 1996.

[25] N. Huber and C. Tsakmakis. Finite deformation viscoelasticity laws. Mechanics of Materials, 32:1-18, 2000.

[26] T.J.R. Hughes. The Finite Element Method. Dover, 2000.

[27] M. Kaliske and H. Rothert. Formulation and implementation of three-dimensional viscoelasticity at small and finite strains. Comput. Mech., 19:228-239, 1997.

[28] J. Keck. Zur Beschreibung finiter Deformationen von Polymeren: Experimente, Modellbildung, Parameteridentifikation und Finite-Elemente-Formulierung. PhD thesis, Institut für Mechanik, Bauwesen, Lehrstuhl I), Universität Stuttgart, Fakultät Bauingenieur- und Vermessungswesen, 1998.

[29] D. Klempner and V. Sendijarevic. Polymeric Foams and Foam Technology. Hanser, 2004.

[30] B. Kleuter, A. Menzel, and P. Steinmann. Generalized parameter identification for finite viscoelasticity. Comput. Methods Appl. Mech. Engrg., 196:3315-3334, 2007.

[31] R. Kreißig, U. Benedix, and U.-J. Görke. Statistical aspects of the identification of material parameters for elasto-plastic models. Arch. Appl. Mech., 71, 2001.

[32] J. Lemaitre and J.-L. Chaboche. Mechanics of Solid Materials. Cambridge, 1998.

[33] R. Leppkes. Polyurethane, volume 91 of Die Bibliothek der Technik. Moderne Industrie, 1993. 
[34] A. Lion. A physically based method to represent the thermo-mechanical behaviour of elastomers. Acta Mech., 123, 1997.

[35] A. Lion. Thixotropic behaviour of rubber under dynamic loading histories: experiments and theory. J. Mech. Phys. Solids, 46, 1998.

[36] A.K. Louis. Inverse und schlecht gestellte Probleme. Teubner, 1989.

[37] J. Lubliner. A model of rubber viscoelasticity. Mech. Res. Comm., 12:93-99, 1985.

[38] D.G. Luenberger. Linear and Nonlinear Programming. Addison-Wesley, 1989.

[39] N. Lundin and A. Klarbring. A Viscoelastic Material Model Including Damagefor describing softening and failure of polymer (PMMA). Lic. thesis, Division of Mechanics, University Linköping, Sweden, 1997.

[40] R. Mahnken. Theoretische und numerische Aspekte zur Parameteridentifikation und Modellierung bei metallischen Werkstoffen. Habilitation, bericht F 98-02, Institut für Baumechanik und Numerische Mechanik, Universität Hannover, Fachbereich Bauingenieur- und Vermessungswesen, 1998.

[41] R. Mahnken. Aspects on the finite-element implementation of the gurson model including parameter identification. Int. J. Plasticity, 15:1111-1137, 1999.

[42] R. Mahnken. A comprehensive study of a multiplicative elastoplasticity model coupled to damage including parameter identification. Comput. Struct., 74:179-200, 2000 .

[43] R. Mahnken. Identification of material parameters for constitutive equations. In E. Stein, R. de Borst, and T.J.R. Hughes, editors, Encyclopedia of Computational Mechanics, volume 2, pages 637-655. Wiley, 2004.

[44] R. Mahnken. Simulation of strength difference for adhesive materials in finite deformation elasto-plasticity. Comput. Methods Appl. Mech. Engrg., 194:4097-4114, 2005 .

[45] R. Mahnken, M. Johansson, and K. Runesson. Parameter estimation for a viscoplastic damage model using a gradient-based optimization algorithm. Engng. Comput., 15(7):925-955, 1998.

[46] R. Mahnken and M. Schlimmer. Simulation of strength difference in elasto-plasticity for adhesive materials. Int. J. Numer. Methods Engng., 63:1461-1477, 2005.

[47] R. Mahnken and E. Stein. Parameter identification for viscoplastic models based on analytical derivatives of a least-squares functional and stability investigations. Int. J. Plasticity, 12:451-479, 1996.

[48] R. Mahnken and E. Stein. A unified approach for parameter identification of inelastic material models in the frame of the finite element method. Comput. Methods Appl. Mech. Engrg., 136:225-258, 1996. 
[49] R. Mahnken and E. Stein. Parameter identification for finite deformation elastoplasticity in principal directions. Comput. Methods Appl. Mech. Engrg., 147:17-39, 1997.

[50] R. Mahnken and P. Steinmann. A finite element algorithm for parameter identification of material models for fluid saturated porous media. Int. J. Numer. Anal. Meth. Geomech., 25:415-434, 2001.

[51] J.E. Marsden and J.R. Hughes. Mathematical Foundations of Elasticity. PrenticeHall, 1983.

[52] A. Menzel. Modelling and Computation of Geometrically Nonlinear Anisotropic Inelasticity. Phd thesis T02-01, Lehrstuhl für Technische Mechanik, Universität Kaiserslautern, Fachbereich Maschinenbau und Verfahrenstechnik, 2002.

[53] A. Menzel, M. Ekh, K. Runesson, and P. Steinmann. A framework for multiplicative elastoplasticity with kinematic hardening coupled to anisotropic damage. Int. J. Plasticity, 21:397-434, 2005.

[54] A. Menzel and P. Steinmann. Geometrically nonlinear anisotropic inelasticity based on fictitious configurations: Application to the coupling of continuum damage and multiplicative elasto-plasticity. Int. J. Numer. Methods Engng., 56:2233-2266, 2003.

[55] A. Menzel and P. Steinmann. On the spatial formulation of anisotropic multiplicative elasto-plasticity. Comput. Methods Appl. Mech. Engrg., 192:3431-3470, 2003.

[56] C. Miehe. Computation of isotropic tensor functions. Cummun. Numer. Methods Engng., 9:889-896, 1993.

[57] C. Miehe and J. Keck. Superimposed finite elastic-viscoelastic-plastoelastic stress response with damage in filled rubbery polymers. experiments, modelling and algorithmic implementation. J. Mech. Phys. Solids, 48:323-365, 2000.

[58] J. Nocedal and S.J. Wright. Numerical Optimization. Springer, 1999.

[59] R. W. Ogden. Non-Linear Elastic Deformations. Dover, 1984.

[60] R.W. Ogden and D.G. Roxburgh. A pseudo-elastic model for the mullins effect in filled rubber. Proc. Roy. Soc. London A, 455:2861-2877, 1999.

[61] M.D.J. Powell. Direct search algorithms for optimization calculations. Acta Numerica, 7:287-336, 1998.

[62] J.N. Reddy. An Introduction to Nonlinear Finite Element Analysis. Oxford University Press, 2004.

[63] S. Reese. Multiplicative thermo-viscoplasticity: A thermodynamic model and its finite element implementation. Technische Mechanik, 18(3):209-216, 1998. 
[64] S. Reese and S. Govindjee. Theoretical and numerical aspects in the thermoviscoelastic material behaviour of rubber-like polymers. Mech. Time-Dependent Mat., 1:357-396, 1998.

[65] S. Reese and S. Govindjee. A theory of finite viscoelasticity and numerical aspects. Int. J. Solids Struct., 35:3455-3482, 1998.

[66] G. Scheday. Theorie und Numerik der Parameteridentifikation von Materialmodellen der finiten Elastizität und Inelastizität auf Grundlage optischer Feldmeßmethoden. Dissertation I-11, Institut für Mechanik (Bauwesen), Lehrstuhl I, Universität Stuttgart, 2003.

[67] G. Scheday and C. Miehe. A unied computational framework for parameter identication of material models in finite inelasticity. PAMM, 1:189-190, 2002.

[68] J.C. Simo. On a fully three-dimensional finite-strain viscoelastic damage model: Formulation and computational aspects. Comput. Methods Appl. Mech. Engrg., 60:153$173,1987$.

[69] J.C. Simo and F. Armero. Geometrically non-linear enhanced strain mixed methods and the method of incompatible modes. Int. J. Numer. Methods Engng., 33:14131449, 1992.

[70] J.C. Simo and T.J.R. Hughes. Computational Inelasticity. Springer, 1998.

[71] P. Spellucci. Numerische Verfahren der nichtlinearen Optimierung. Birkhäuser, 1993.

[72] L.R.G. Treloar. The Physics of Rubber Elasticity. Oxford University Press, 2005.

[73] G. Weber and L. Anand. Finite deformation constitutive equations and a time integration procedure for isotropic hyperelastic-viscoplastic solids. Comput. Methods Appl. Mech. Engrg., 79:173-202, 1990.

[74] D. Winter. Optische Verschiebungsmessung nach dem Objektrasterprinzip mit Hilfe eines flächenorientierten Ansatzes. Phd thesis, Lehrstuhl für Technische Mechanik, Universität Braunschweig, 1993.

[75] P. Wriggers. Nichtlineare Finite-Element-Methoden. Springer, 2001.

[76] O.C. Zienkewicz and R.L. Taylor. The Finite Element Method, Volume I: The Basis. Butterworth Heinemann, 2000.

[77] O.C. Zienkewicz and R.L. Taylor. The Finite Element Method, Volume II: Solid Mechanics. Butterworth Heinemann, 2000. 


\section{Curriculum Vitae}

Name:

Adress:

Date and place of birth:

$1981-1985$

$1985-06 / 1994$

$04 / 1993-04 / 2000$

$11 / 1994-10 / 1997$

$10 / 1997-05 / 2003$

since $6 / 2003$
Bernd Kleuter

Konrad-Adenauer-Strasse 7, D-67663 Kaiserslautern

29.04.1975, Düsseldorf

Grundschule Erkrath

Gymnasium am Neandertal, Erkrath

Alternative civilian service at the German Red Cross

Self-employed as sound engineer

Mechanical engineering, University of Kaiserslautern

Research associate, University of Kaiserslautern

Chair of Applied Mechanics 